\title{
A Hydrogen Peroxide Complex of Zinc
}

Christian M. Wallen, John Bacsa, and Christopher C. Scarborough*

Emory University, Department of Chemistry, 1515 Dickey Drive, Atlanta, GA 30322, U.S.A.

Supporting Information 
Table of Contents

1. Materials and Methods S3

2. Synthesis and Characterization S4

$\begin{array}{ll}\text { 3. NMR Experiments } & \text { S7 }\end{array}$

4. Equilibrium Calculation $\quad$ S13

5. Gravimetric Analysis $\quad$ S14

$\begin{array}{lr}\text { 6. NMR Spectra } & \text { S18 }\end{array}$

$\begin{array}{lr}\text { 7. IR Spectra } & \text { S29 }\end{array}$

8. Crystallographic information $\quad \mathbf{S 3 0}$

$\begin{array}{lll}\text { 9. } & \text { References } & \text { S88 }\end{array}$ 


\section{Materials and Methods}

All room temperature ${ }^{1} \mathrm{H}-\mathrm{NMR}$ and ${ }^{13} \mathrm{C}-\mathrm{NMR}$ spectra were obtained on a $400 \mathrm{MHz}$ instrument and referenced to residual solvent. Low-temperature NMR experiments were conducted on a $600 \mathrm{MHz}$ NMR spectrometer and referenced to residual solvent. IR spectra were obtained using a Nicolet 380 FT-IR with Smart Orbit Diamond ATR attachment. X-ray crystallography data was collected and analyzed by the Emory University Department of Chemistry X-Ray Crystallography Center. Elemental analyses were performed by Atlantic Microlabs in Norcross, GA. All reactions sensitive to air and/or moisture were carried out in an MBraun UNIlab glovebox under nitrogen atmosphere. Solvents for use in the glovebox were degassed by evacuation and purging with nitrogen and dried using $3 \AA ̊$ molecular sieves. Anhydrous tetrahydrofuran was prepared in the glovebox by storing over $3 \AA$ molecular sieves for several days and then filtering through a column of activated alumina to remove trace water and peroxides. THF- $\mathrm{d}_{8}$ was purchased from Cambridge Isotope Laboratories and dried using $3 \AA ̊$ molecular sieves. Reagents were purchased from Sigma-Aldrich, Fisher Scientific, Acros Organics, or Strem Chemicals and used without further purification.

\section{Extraction of $\mathrm{H}_{2} \mathrm{O}_{2}$ in THF:}

Urea hydrogen peroxide was stirred in anhydrous THF for $30 \mathrm{~min}$. The solution was filtered through celite and cooled to $-40^{\circ} \mathrm{C}$ for several hours to crystalize dissolved urea. The solution was filtered through celite again to remove urea crystals. Iodometric titration was used to determine the peroxide concentration in this 
solution. The same procedure was used to extract hydrogen peroxide into THF- $\mathrm{d}_{8}$. ${ }^{1} \mathrm{H}$ NMR spectroscopy was used to ensure that the solution contained $<5 \%$ water.

\section{Synthesis and Characterization}

\section{$\mathrm{H}_{3} \mathrm{Ts}_{3}$ tren:}

Ligand precursor was synthesized according to a modified literature procedure. ${ }^{1} \mathrm{~A}$ solution of p-toluenesulfonyl chloride $(11.64 \mathrm{~g}, 0.061 \mathrm{~mol})$ dissolved in $100 \mathrm{~mL}$ of diethyl ether was added to a solution of triethylamine $(8.4 \mathrm{~mL}, 0.060 \mathrm{~mol})$ and tris $(2$ aminoethyl)amine $(2.9 \mathrm{~mL}, 0.019 \mathrm{~mol})$ in $80 \mathrm{~mL}$ of deionized water. The reaction mixture was stirred vigorously for $14 \mathrm{hrs}$. The white solid was filtered from the reaction mixture, washed with water $(100 \mathrm{~mL})$ and diethyl ether $(300 \mathrm{~mL})$. Drying under vacuum yielded pure product $(11.0 \mathrm{~g}, 93 \%$ yield $)$. Characterization data agreed with published material. ${ }^{1}{ }^{1} \mathrm{H}$ NMR $\left(400 \mathrm{MHz}, \mathrm{CDCl}_{3}\right): \delta 7.77(\mathrm{~d}, 6 \mathrm{H}, \mathrm{J}=8.3 \mathrm{~Hz}$, Ar-H), 7.26 (d, 6Hf, J=8.3Hz), $5.85(\mathrm{t}, 3 \mathrm{H}), 2.89(\mathrm{~m}, 6 \mathrm{H}), 2.45(\mathrm{~m}, 6 \mathrm{H}), 2.39(\mathrm{~s}, 9 \mathrm{H}) ;{ }^{13} \mathrm{C}$ NMR (100 Hz, $\left.\mathrm{CDCl}_{3}\right): \delta 143.8,137.2,130.2,127.6,54.5,41.2,22.0$.

\section{Synthesis and Characterization of $\left[\mathrm{Bu}_{4} \mathrm{~N}\right]\left[\mathrm{Ts} s_{3} \operatorname{trenZn-}-\mathrm{OH}_{2}\right]\left(1-\mathrm{OH}_{2}\right)$ :}

In a glovebox, $\mathrm{ZnCl}_{2}(1.16 \mathrm{~g}, 8.54 \mathrm{mmol})$ was added to a stirring solution of $\mathrm{H}_{3} \mathrm{Ts}_{3}$ tren (5.20g, $8.54 \mathrm{mmol})$ in $15 \mathrm{~mL}$ of DMF. Potassium hydride $(1.06 \mathrm{~g}, 26.48 \mathrm{mmol})$ was added carefully and the mixture was stirred overnight. The solution was removed from the glovebox and tetrabutylammonium bromide $(2.67 \mathrm{~g}, 8.54 \mathrm{mmol})$ was added. The mixture was diluted with water $(200 \mathrm{~mL})$ and DCM $(200 \mathrm{~mL})$. The organic layer was separated and washed with more water $(200 \mathrm{~mL})$ then brine $(200 \mathrm{~mL})$. The organic layer was then dried with $\mathrm{Na}_{2} \mathrm{SO}_{4}$, filtered, and concentrated under reduced pressure. Addition of ether into this concentrated solution resulted in formation of 
colorless crystals $\left(7.29 \mathrm{~g}, 92 \%\right.$ yield). ${ }^{1} \mathrm{H}$ NMR $\left(400 \mathrm{MHz}, \mathrm{CDCl}_{3}\right): \delta 7.79(\mathrm{~d}, 6 \mathrm{H}$, $\mathrm{J}=8.2 \mathrm{~Hz}), 7.12(\mathrm{~d}, 6 \mathrm{H}, \mathrm{J}=8.2 \mathrm{~Hz}), 6.63(\mathrm{br} \mathrm{s}, 2 \mathrm{H}), 3.27(\mathrm{~m}, 8 \mathrm{H}), 2.88(\mathrm{t}, 6 \mathrm{H}, \mathrm{J}=5.4 \mathrm{~Hz})$, $2.33(\mathrm{~s}, 9 \mathrm{H}), 2.29(\mathrm{t}, 6 \mathrm{H}, \mathrm{J}=5.5 \mathrm{~Hz}), 1.64(\mathrm{~m}, 8 \mathrm{H}), 1.40(\mathrm{~m}, 8 \mathrm{H}), 0.96(\mathrm{t}, 12 \mathrm{H}, \mathrm{J}=7.3 \mathrm{~Hz})$. ${ }^{13} \mathrm{C}$ NMR $\left(100 \mathrm{~Hz}, \mathrm{CDCl}_{3}\right): \delta$ 142.0, 140.1, 129.1, 127.2, 58.7, 52.5, 42.1, 24.1, 21.4, 19.8, 13.9. Anal. calcd (found) for $\left(\mathrm{C}_{43} \mathrm{H}_{71} \mathrm{~N}_{5} \mathrm{O}_{7} \mathrm{~S} 3 \mathrm{Zn}\right)$ : C, 55.44 (55.65); H, 7.68 (7.70); N, 7.52 (7.42). IR ( $\left.\mathrm{cm}^{-1}\right): 3296,2961,2873,1598,1494,1466,1382,1351,1270$, $1240,1158,1128,1103,1077,1046,974,930,871,846,812,738,710,657,595$, 549.

\section{Synthesis and Characterization of $\left[\mathrm{Bu}_{4} \mathrm{~N}\right]\left[\mathrm{Ts}_{3} \operatorname{trenZn}-\mathrm{N}_{2} \mathrm{H}_{4}\right]\left(1-\mathrm{N}_{2} \mathrm{H}_{4}\right)$ :}

Hydrazine solution $(220 \mu \mathrm{L}, 1.0 \mathrm{M}$ in THF, $0.22 \mathrm{mmol})$ and $\mathbf{1}-\mathbf{O H}_{2}(102 \mathrm{mg}, 0.11 \mathrm{mmol})$ was added to $0.5 \mathrm{~mL}$ of DCM. Vapor diffusion of diethyl ether into this solution produced colorless crystals $\left(72 \mathrm{mg}, 76 \%\right.$ yield). ${ }^{1} \mathrm{H}$ NMR $\left(400 \mathrm{MHz}, \mathrm{CDCl}_{3}\right): \delta 7.83(\mathrm{~d}$, $6 \mathrm{H}, \mathrm{J}=8.1 \mathrm{~Hz}), 7.15(\mathrm{~d}, 6 \mathrm{H}, \mathrm{J}=8.1 \mathrm{~Hz}), 4.86(\mathrm{br} \mathrm{s}, 2 \mathrm{H}), 4.10(\mathrm{br} \mathrm{s}, 2 \mathrm{H}), 3.28(\mathrm{~m}, 8 \mathrm{H}), 2.86$ (t, 6H, J=5.3Hz), $2.35(\mathrm{~s}, 9 \mathrm{H}), 2.27(\mathrm{t}, 6 \mathrm{H}, \mathrm{J}=5.3 \mathrm{~Hz}), 1.63(\mathrm{~m}, 8 \mathrm{H}), 1.41(\mathrm{~m}, 8 \mathrm{H}), 0.97(\mathrm{t}$, $12 \mathrm{H}, \mathrm{J}=7.3 \mathrm{~Hz}) .{ }^{13} \mathrm{C}$ NMR $\left(100 \mathrm{~Hz}, \mathrm{CDCl}_{3}\right): \delta 142.8,140.0,129.0,127.0,58.4,42.6$, 24.1, 21.5, 19.8, 13.9. IR ( $\left.\mathrm{cm}^{-1}\right): 3260,3329,3295,2955,2874,1598,1537,1493$, $1467,1382,1351,1285,1245,1243,1129,1098,1079,1045,984,971,930,870$, 846, 813, 794, 739, 710, 659, 592, 548. Anal. calcd (found) for $\left(\mathrm{C}_{43} \mathrm{H}_{72} \mathrm{~N}_{7} \mathrm{O}_{6} \mathrm{~S}_{3} \mathrm{Zn}\right)$ : C, 54.67 (54.86); H, 7.68 (7.81); N, 10.38 (10.17).

\section{Synthesis and Characterization of $\left[\mathrm{Bu}_{4} \mathrm{~N}\right]\left[\mathrm{Ts}_{3} \operatorname{trenZn}-\mathrm{NH}_{2} \mathrm{OH}\right]\left(1-\mathrm{NH}_{2} \mathrm{OH}\right)$ :}

Hydroxylamine solution ( $42 \mathrm{mg}$, $0.63 \mathrm{mmol}, 50 \mathrm{wt} \%$ in $\mathrm{H}_{2} \mathrm{O}$ ) was diluted with $1 \mathrm{~mL}$ of 1:1 THF and $\mathrm{MeOH}$, and added to $\mathbf{1}-\mathbf{O H}_{\mathbf{2}}(118 \mathrm{mg}, 0.127 \mathrm{mmol})$. This solution was stirred until everything dissolved and then filtered through celite. Vapor diffusion of 
diethyl ether into this solution yielded colorless crystals (88 mg, 79\% yield). ${ }^{1} \mathrm{H}$ NMR (400 MHz, $\mathrm{CDCl}_{3}$ ): $\delta 8.33$ (br s, $1 \mathrm{H}$ ), 7.79 (d, 6H, J=8.1Hz), 7.18 (br s, 2H), 7.12 (d, 6H, J=8.1Hz), $3.25(\mathrm{~m}, 8 \mathrm{H}), 2.81(\mathrm{t}, 6 \mathrm{H}, \mathrm{J}=5.2 \mathrm{~Hz}), 2.33(\mathrm{~s}, 9 \mathrm{H}), 2.22(\mathrm{t}, 6 \mathrm{H}$, $\mathrm{J}=5.2 \mathrm{~Hz}), 1.61(\mathrm{~m}, 8 \mathrm{H}), 1.41(\mathrm{~m}, 8 \mathrm{H}), 0.96(\mathrm{t}, 12 \mathrm{H}, \mathrm{J}=7.8 \mathrm{~Hz}) \cdot{ }^{13} \mathrm{C} \mathrm{NMR}\left(100 \mathrm{~Hz}, \mathrm{CDCl}_{3}\right):$ $\delta 142.8,140.0,129.0,127.0,58.54,54.6,42.7,24.1,21.4,19.8,13.9 . \operatorname{IR}\left(\mathrm{cm}^{-1}\right): 3289$, $2963,2874,1598,1494,1466,1383,1352,1286,1245,1129,1097,1079,1045$, 970, 932, 871, 846, 812, 793, 738, 696, 659, 591, 547. Anal. calcd (found) for ( $\left.\mathrm{C}_{43} \mathrm{H}_{72} \mathrm{~N}_{6} \mathrm{O}_{7} \mathrm{~S}_{3} \mathrm{Zn}\right): \mathrm{C}, 54.56$ (54.81); H, 7.67 (7.46); N, 8.88 (9.00).

\section{Synthesis and Characterization of $\left[\mathrm{Bu}_{4} \mathrm{~N}\right]\left[\mathrm{Ts}_{3} \operatorname{trenZn}\right](1)$ :}

In the glovebox, $\mathbf{1 -}-\mathbf{O H}_{2}(347 \mathrm{mg})$ was dissolved in 1,2-dimethoxyethane $(5 \mathrm{~mL})$ and dehydrated $4 \AA ̊$ molecular sieves were added. The vessel was sealed and heated to $80^{\circ} \mathrm{C}$ overnight. Pentane was added to precipitate the $\left[\mathrm{Bu}_{4} \mathrm{~N}\right]\left[\mathrm{Ts}_{3}\right.$ trenZn $]$ complex $(\mathbf{1}$, 288mg, 85\% yield). ${ }^{1} \mathrm{H}$ NMR (400 MHz, $\left.\mathrm{CDCl}_{3}\right): \delta 8.01(\mathrm{~d}, 6 \mathrm{H}, \mathrm{J}=8.1 \mathrm{~Hz}), 7.21(\mathrm{~d}, 6 \mathrm{H}$, $\mathrm{J}=8.1 \mathrm{~Hz}), 3.14(\mathrm{~m}, 8 \mathrm{H}), 2.92(\mathrm{t}, 6 \mathrm{H}, \mathrm{J}=5.5 \mathrm{~Hz}), 2.43(\mathrm{t}, 6 \mathrm{H}, \mathrm{J}=5.5 \mathrm{~Hz}), 2.35(\mathrm{~s}, 9 \mathrm{H}), 1.49$ $(\mathrm{m}, 8 \mathrm{H}), 1.17(\mathrm{~m}, 8 \mathrm{H}), 0.76(\mathrm{t}, 12 \mathrm{H}, \mathrm{J}=7.8 \mathrm{~Hz}) ;{ }^{13} \mathrm{C} \mathrm{NMR}\left(100 \mathrm{~Hz}, \mathrm{CDCl}_{3}\right): \delta$ 142.0, $140.1,129.1,127.2,58.66,52.5,42.1,24.1,21.4,19.8,13.9$.

\section{Crystallization of $\left[\mathrm{Bu}_{4} \mathrm{~N}\right]\left[\mathrm{Ts}_{3} \operatorname{trenZn}-\mathrm{H}_{2} \mathrm{O}_{2}\right]\left(1-\mathrm{H}_{2} \mathrm{O}_{2}\right)$ :}

In the glovebox, $\mathbf{1}$ was dissolved in THF and 1 molar equivalent of $\mathrm{H}_{2} \mathrm{O}_{2}$ solution in THF was added. Layering of this solution under tert-butyl methyl ether at room temperature yielded colorless crystals within a few hours. The ${ }^{1} \mathrm{H}$ NMR spectrum of a sample of crystals indicated the presence of $44 \% \mathrm{Zn}-\mathrm{H}_{2} \mathrm{O}_{2}$ complex and $58 \% \mathrm{Zn}$ $\mathrm{OH}_{2}$ complex. Single crystals were selected and mounted on the diffractometer, and 
the crystal with the highest $\mathbf{1}-\mathbf{H}_{2} \mathbf{O}_{2}$ content $(50 \%)$ was selected for full crystallographic analysis.

\section{NMR Experiments}

\section{${ }^{1} \mathrm{H}-\mathrm{NMR}$ spectrum of $1-\mathrm{H}_{2} \mathrm{O}_{2}$ decay in THF-d8:}

In a glovebox, $19.2 \mathrm{mg}$ of $\mathbf{1}$ and one molar equivalent of hydrogen peroxide $(152 \mu \mathrm{L}$ of $138 \mathrm{mM}$ solution in THF- $\mathrm{d}_{8}$ ) were added to a J. Young NMR tube and diluted with THF- $\mathrm{d}_{8}$ to $11 \mathrm{mM}$. The sample was analyzed over the course of $14 \mathrm{hrs}$ at $20^{\circ} \mathrm{C}$. Spectra were taken every 21.7 minutes; taking 8 scans with a relaxation delay of 30sec.

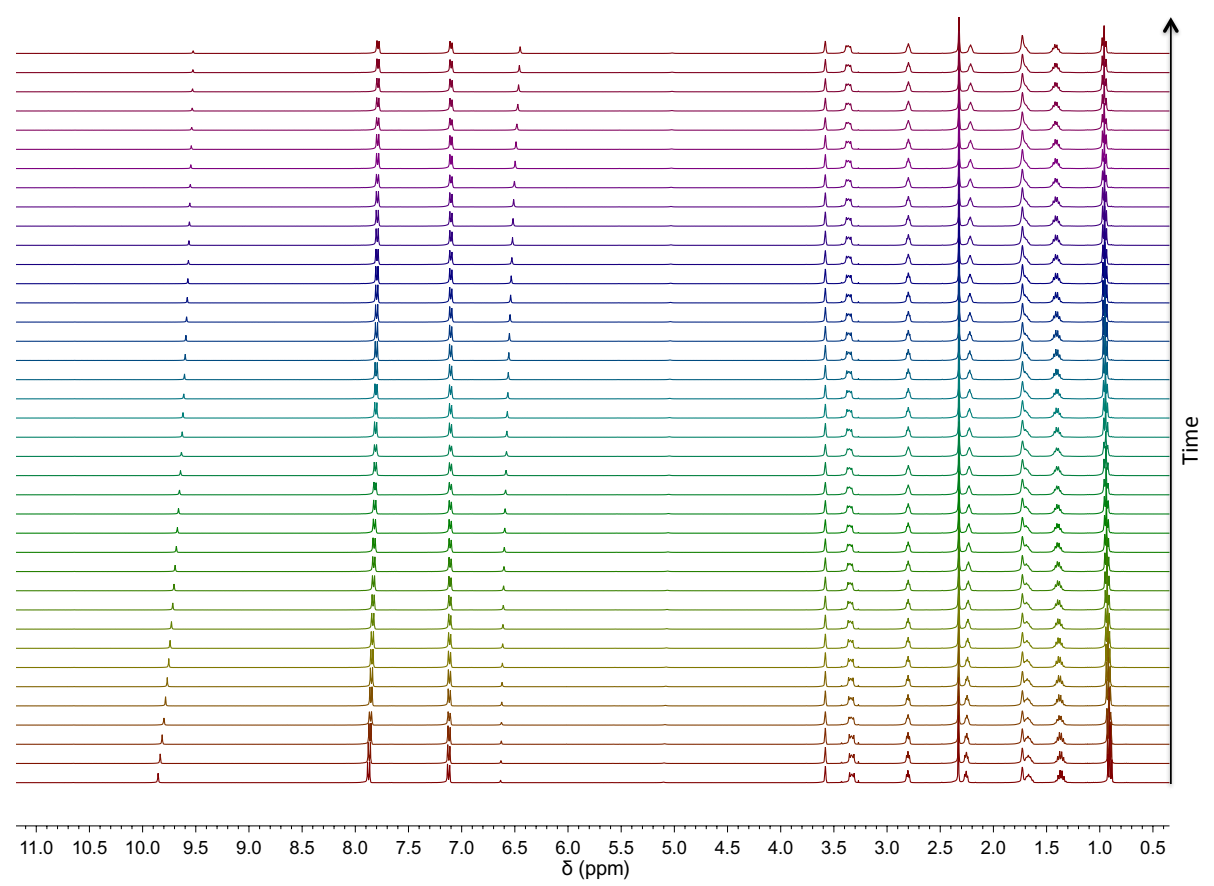

Figure S1. ${ }^{1} \mathrm{H}-\mathrm{NMR}$ of $1-\mathrm{H}_{2} \mathrm{O}_{2}$ decay into $1-\mathrm{OH}_{2}$ in THF-d 8 .

\section{Decay of $\mathrm{H}_{2} \mathrm{O}_{2}$ in THF-d 8 :}

In a glovebox, $280 \mu \mathrm{L}$ of $138 \mathrm{mM}$ solution in THF- $\mathrm{d}_{8}$ was added to J. Young tube and diluted with THF- $\mathrm{d}_{8}$ to $22 \mathrm{mM}$. The sample was analyzed over the course of $14 \mathrm{hrs}$ at 
$20^{\circ} \mathrm{C}$. Spectra were taken every 21.7 minutes; taking 8 scans with a relaxation delay of 30sec.
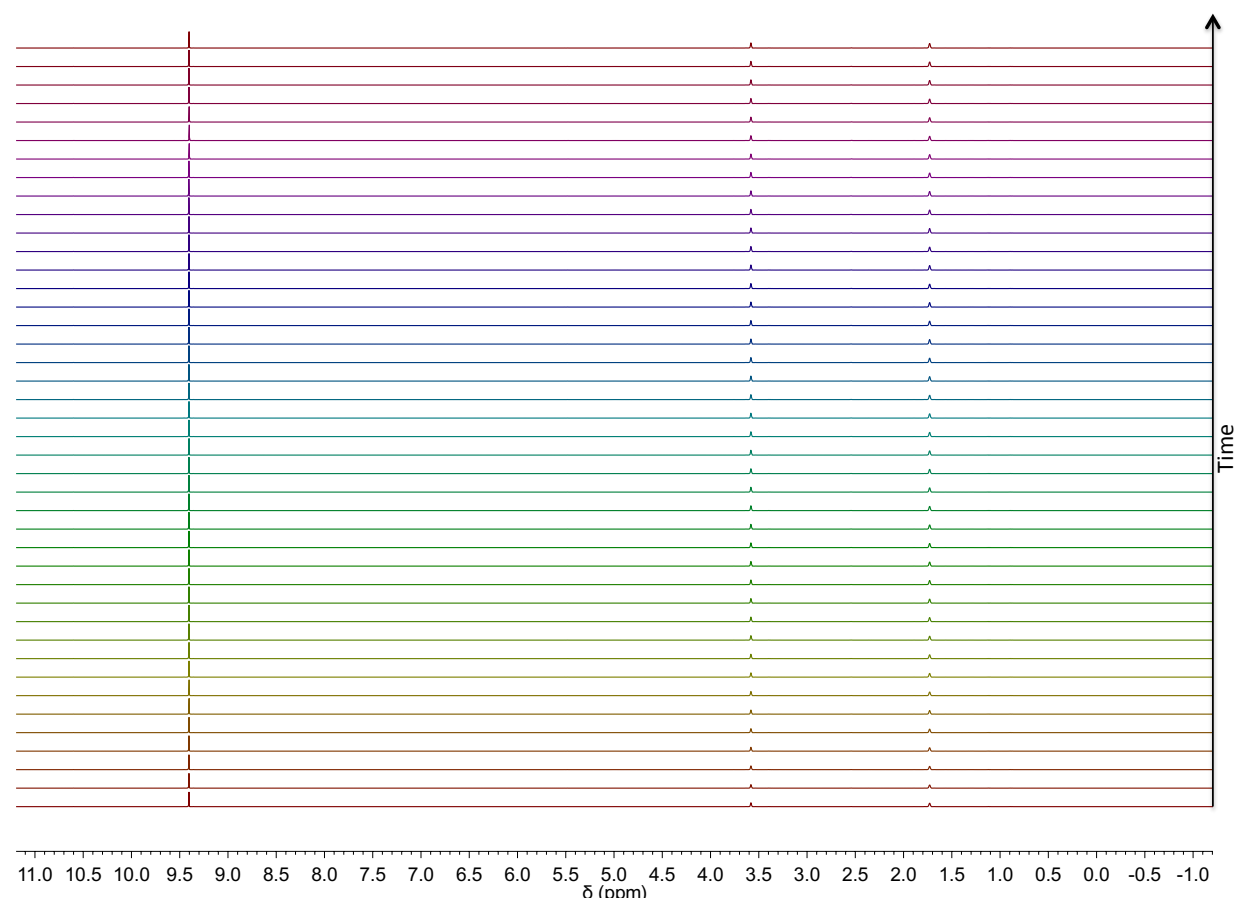

Figure S2. ${ }^{1} \mathrm{H}-\mathrm{NMR}$ of $\mathrm{H}_{2} \mathrm{O}_{2}$ decay in THF-d 8 .

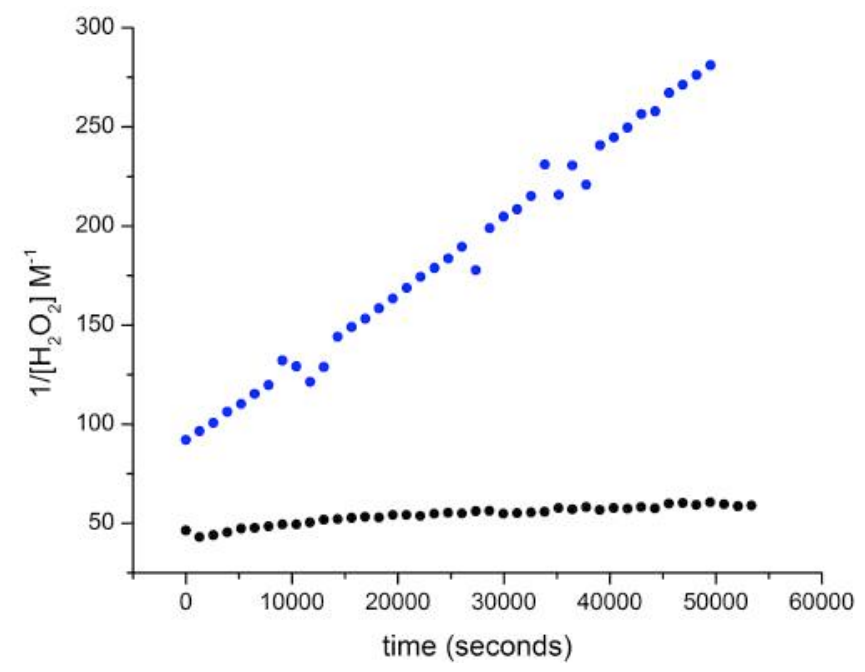

Figure S3. Second-order decay of $1-\mathrm{H}_{2} \mathrm{O}_{2}$ (blue) with $\mathrm{k}=3.8 \times 10^{-3} \mathrm{M}^{-1} \mathrm{~s}^{-1}$ and $\mathrm{H}_{2} \mathrm{O}_{2}$ (black) with $\mathrm{k}=2.7 \times 10^{-4} \mathrm{M}^{-1} \mathrm{~s}^{-1}$. Fit (blue): $\mathrm{y}=0.003840 \mathrm{x}+88.597934\left(\mathrm{R}^{2}\right.$ 
$=0.99163)$. Fit (black): $y=0.000275 x+46.750288\left(R^{2}=0.89763\right)$ (fit as

second-order decay only for comparison to $1-\mathrm{H}_{2} \mathrm{O}_{2}$ data).

${ }^{1} \mathrm{H}-\mathrm{NMR}$ of $\mathrm{H}_{3} \mathrm{Ts}_{3}$ tren and $\mathrm{H}_{2} \mathrm{O}_{2}$ in THF-d

In a glovebox, $\mathrm{H}_{3} \mathrm{Ts}_{3}$ tren $(14 \mathrm{mg})$ and $\mathrm{H}_{2} \mathrm{O}_{2}(171 \mu \mathrm{L}$ of $138 \mathrm{mM}$ solution) were dissolved in THF-d 8 .

${ }^{1} \mathrm{H}-\mathrm{NMR}$ of $\mathrm{ZnCl}_{2}$ and $\mathrm{H}_{2} \mathrm{O}_{2}$ in THF-d 8 :

In a glovebox, $\mathrm{ZnCl}_{2}(6.41 \mathrm{mg})$ and $\mathrm{H}_{2} \mathrm{O}_{2}(70 \mu \mathrm{L}$ of $671 \mathrm{mM}$ solution) were dissolved in THF-d8.

\begin{tabular}{|c|c|c|}
\hline Sample & $\begin{array}{l}\text { Signal } \\
\text { (ppm) }\end{array}$ & $\begin{array}{l}\text { Shift from free } \\
\text { position (ppm) }\end{array}$ \\
\hline $\mathrm{H}_{2} \mathrm{O}_{2}$ & 9.391 & -- \\
\hline $\mathrm{ZnCl}_{2}+\mathrm{H}_{2} \mathrm{O}_{2}$ & 9.386 & -0.005 \\
\hline $\mathrm{H}_{3} \mathrm{Ts} s_{3}$ tren $+\mathrm{H}_{2} \mathrm{O}_{2}$ & 9.399 & 0.008 \\
\hline $\mathbf{1}-\mathbf{O H}_{\mathbf{2}}+\mathrm{H}_{2} \mathrm{O}_{2}$ & 9.455 & 0.064 \\
\hline $1+\mathrm{H}_{2} \mathrm{O}_{2}$ & 9.854 & 0.463 \\
\hline
\end{tabular}

Table S1. Shift of hydrogen peroxide proton signal in THF-d . $_{\text {. }}$

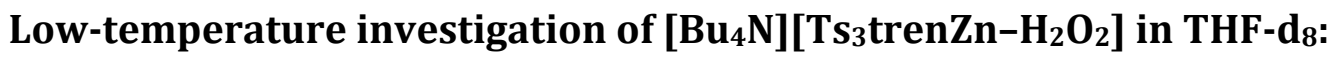

In a glovebox, $20.9 \mathrm{mg}$ of $\mathbf{1}$ and $\mathrm{H}_{2} \mathrm{O}_{2}\left(86 \mu \mathrm{L}\right.$ of $266 \mathrm{mM}$ solution in THF- $\left.\mathrm{d}_{8}\right)$ were added to a J. Young NMR tube and diluted with THF- $\mathrm{d}_{8}$ to $19 \mathrm{mM}$. ${ }^{1} \mathrm{H}$ NMR spectra were taken at the following temperatures: $293,253,243,233,223,213,208,203$, 198, 193, 188, 183, 178, and 173K. Each spectrum comprised of 8 scans with a relaxation delay of 30sec. Close-up spectra of upfield and downfield regions provided in NMR Spectra section. 


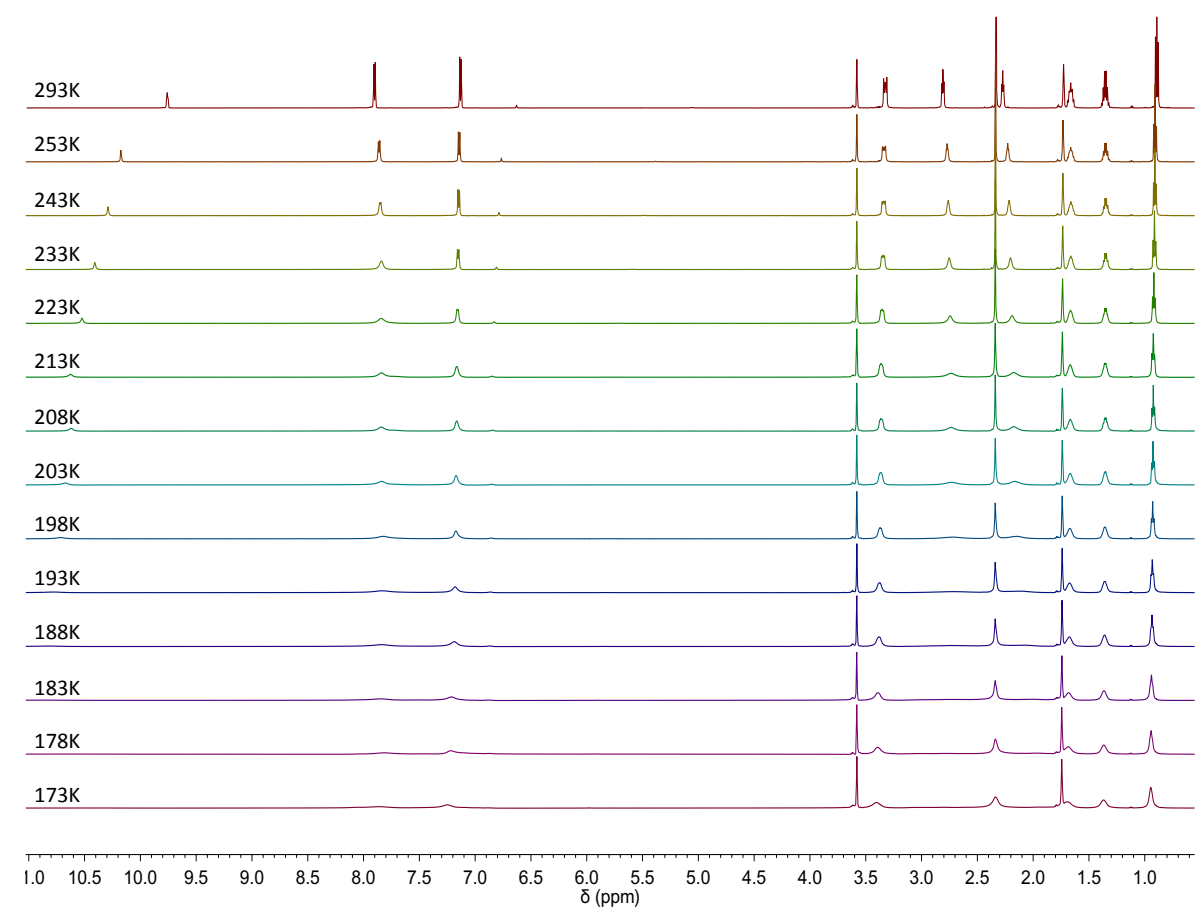

Figure S4. Variable-Temperature ${ }^{1} \mathrm{H}-\mathrm{NMR}$ Spectra of $1-\mathrm{H}_{2} \mathrm{O}_{2}$ in THF-d 8 .

\section{Low-temperature investigation of $\mathrm{H}_{2} \mathrm{O}_{2}$ in THF-d8:}

In a glovebox, $90 \mu \mathrm{L}$ of $266 \mathrm{mM}$ solution of hydrogen peroxide in THF- $\mathrm{d}_{8}$ was added to a J. Young NMR tube and diluted with THF- $\mathrm{d}_{8}$ to $46 \mathrm{mM}$. The tube was sealed and the sample placed in the instrument. Spectra were taken at the following temperatures: 293, 223, 213, 203, 193, and 183K. Each spectrum comprised of 8 scans with a relaxation delay of 30 sec. Close-up spectra of upfield and downfield regions provided in NMR Spectra section. 

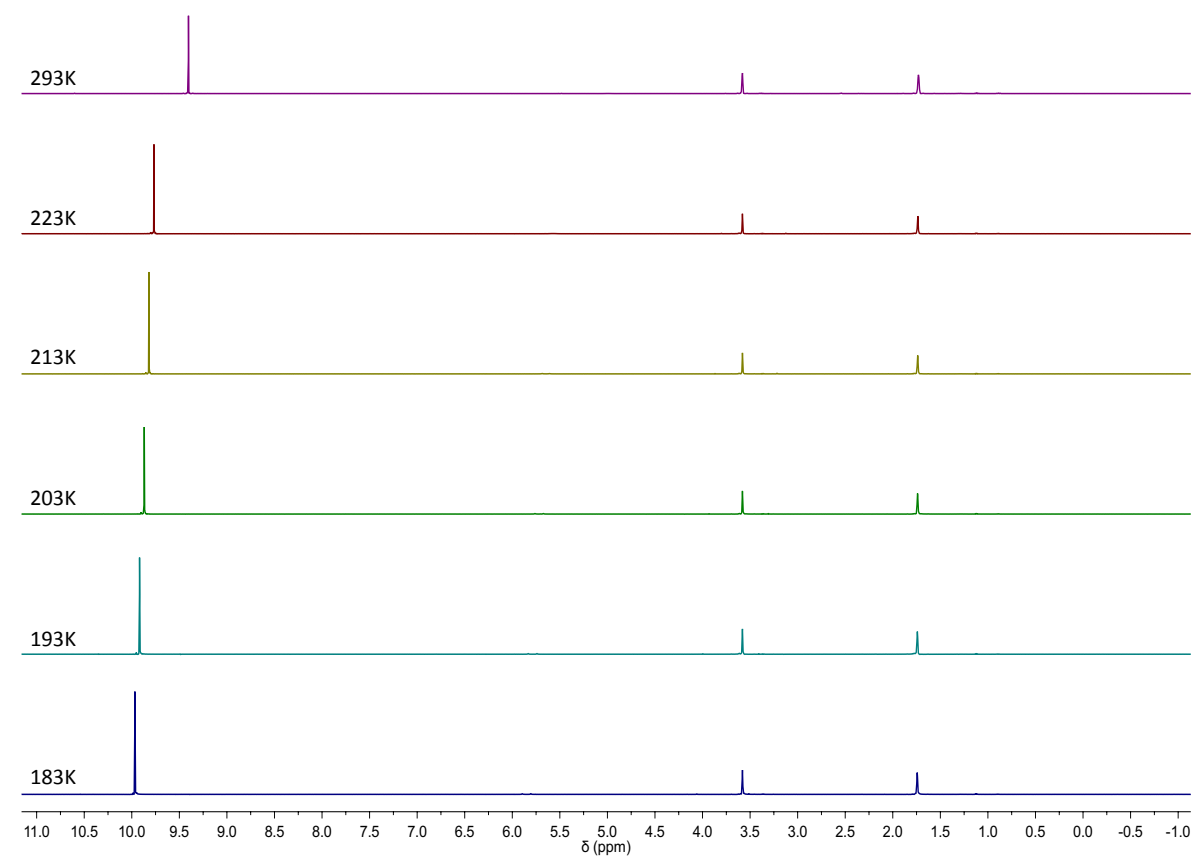

Figure S5. Variable-Temperature ${ }^{1} \mathrm{H}-\mathrm{NMR}$ Spectra of $\mathrm{H}_{2} \mathrm{O}_{2}$ in THF-d8.

Low-temperature investigation of $\left[\mathrm{Bu}_{4} \mathrm{~N}\right]\left[\mathrm{Ts}_{3} \operatorname{trenZn-O\mathrm {H}_{2}]}\left(1-\mathrm{OH}_{2}\right)\right.$ and $\left[\mathrm{Bu}_{4} \mathrm{~N}\right]\left[\mathrm{Ts}_{3}\right.$ trenZn] (1) in THF-d

In the glovebox, a sample of $\mathbf{1}-\mathbf{O H}_{\mathbf{2}}$ was partially dehydrated by heating under vacuum. A portion (18.8mg) of this solid was dissolved in THF- $\mathrm{d}_{8}$ and placed in a J. Young tube. Room temperature NMR spectroscopy indicated the presence of $30 \%$ water bound to Zn. Spectra were taken at the following temperatures: 223, 213, 208, $203,198,193,188,183,178$, and 173K. Each spectrum comprised of 8 scans with a relaxation delay of 30 sec. 


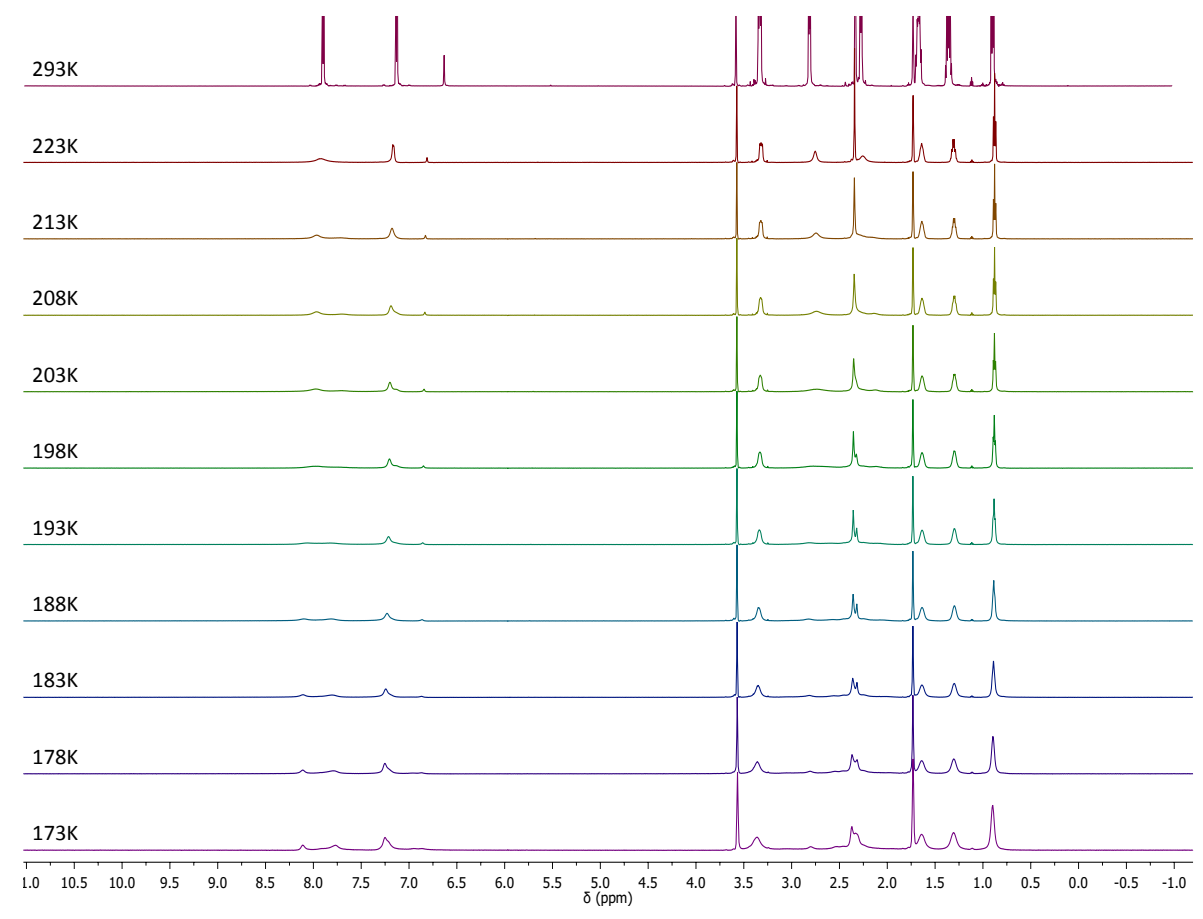

Figure S6. Variable-Temperature ${ }^{1} \mathrm{H}-\mathrm{NMR}$ Spectra of 1-OH $\mathrm{H}_{2}$ in THF-d8.

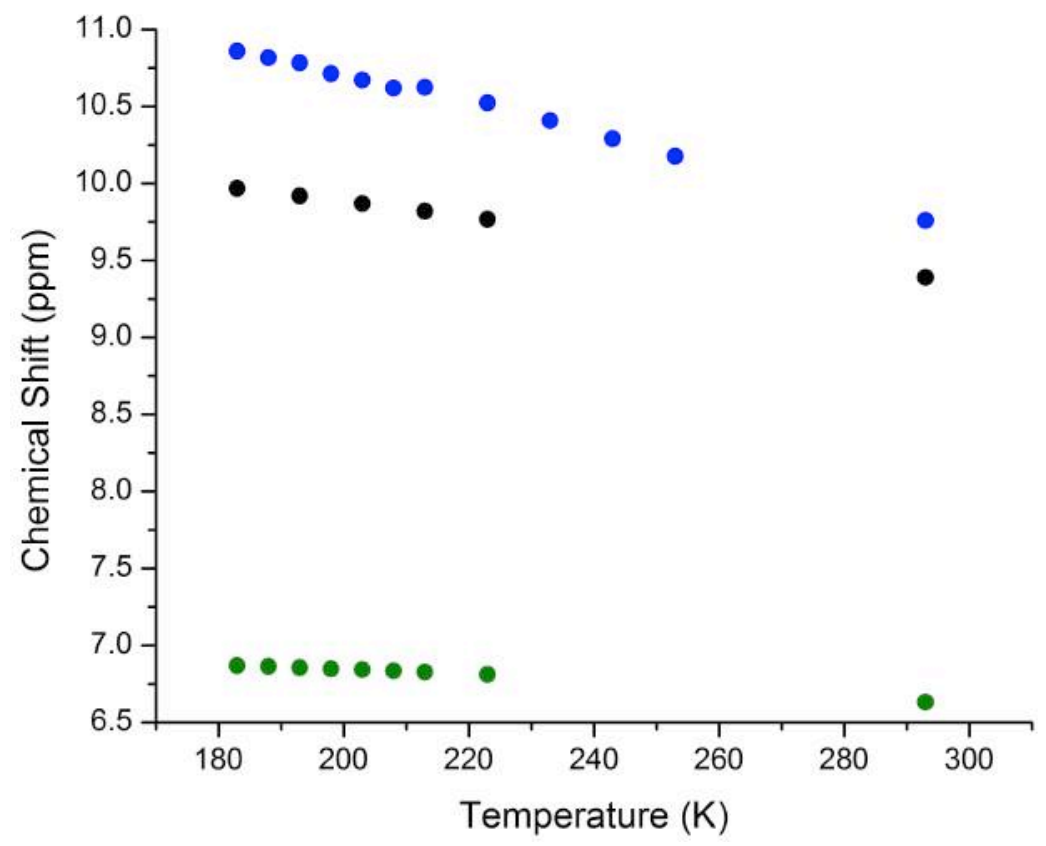

Figure S7. Temperature Dependence of Chemical Shift of Proton Signal for Bound $\mathrm{H}_{2} \mathrm{O}_{2}$ (blue), Free $\mathrm{H}_{2} \mathrm{O}_{2}$ (black), and Bound Water (green). Fit (blue): $\mathrm{y}=$ $-0.009960 x+12.704304\left(R^{2}=0.994524\right)$. Fit $($ black $): y=-$ 
$0.005261 x+10.935487\left(R^{2}=0.999549\right)$. Fit (green): $y=-0.002178 x+7.281224$

$$
\left(R^{2}=0.980630\right) \text {. }
$$

\section{Competition of peroxide and water binding to zinc complex 1:}

In a glovebox, $23.0 \mathrm{mg}$ of $\mathbf{1 - 0 \mathbf { H } _ { 2 }}$ and 1 molar equivalent of hydrogen peroxide $(36 \mu \mathrm{L}$ of $700 \mathrm{mM}$ solution in THF- $\mathrm{d}_{8}$ ) were added to a J. Young tube with THF- $\mathrm{d}_{8}$. A spectrum was taken at room temperature ( 8 scans, relaxation delay $=30 \mathrm{~s})$.

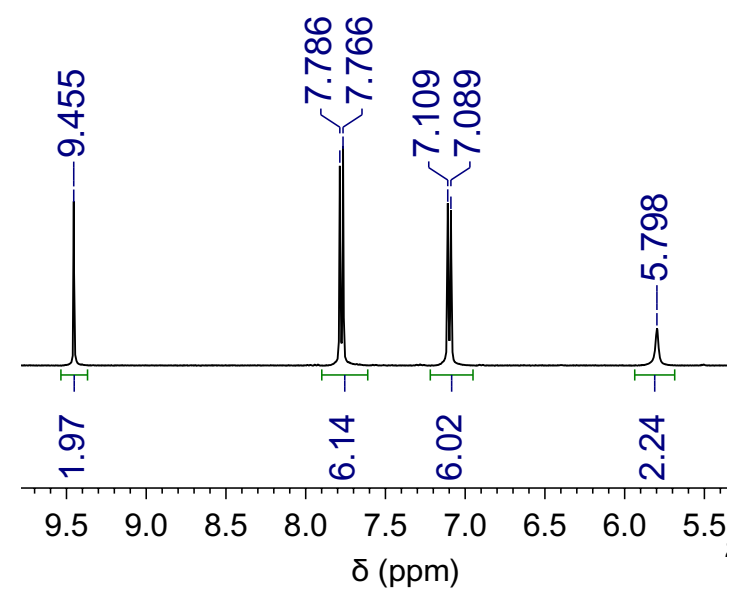

Figure S8. ${ }^{1} \mathrm{H}-\mathrm{NMR}$ spectrum of $1-\mathrm{OH}_{2}$ and $1-\mathrm{H}_{2} \mathrm{O}_{2}$ equilibrium measurement in

THF-d8.

\section{Equilibrium Calculation}

In the competition spectrum mentioned above, the integration of the sulfonamidate ligand was set to 1 equivalent and the integration and position of both the water signals and the peroxide signals were used with the following equations to calculate $\mathrm{K}_{\mathrm{eq}}$ for the competitive binding:

$$
\begin{gathered}
\left(I_{\text {Exp }}\right)\left(S_{\text {Exp }}\right)=\left(I_{\text {Bound }}\right)\left(S_{\text {Bound }}\right)+\left(I_{\text {Free }}\right)\left(S_{\text {Free }}\right) \\
\left(I_{\text {Exp }}\right)=\left(I_{\text {Bound }}\right)+\left(I_{\text {Free }}\right)
\end{gathered}
$$


where $I_{E x p}$ and $S_{E x p}$ are the integration and chemical shift, respectively, of the ligand (water or peroxide) measured in the competition spectrum, $S_{\text {Free }}$ and $S_{\text {Bound }}$ are the chemical shifts of the ligand in its free position and when it is bound to the zinc complex, respectively, and $I_{\text {Bound }}$ and $I_{\text {Free }}$ are the calculated equivalents of ligand bound to the zinc complex and free in solution, respectively. These values are then used in the following equations:

$$
\begin{gathered}
K_{\text {eq }}=\frac{\left[I_{\text {BoundPeroxide }}\right]\left[I_{\text {FreeWater }}\right]}{\left[I_{\text {BoundWater }}\right]\left[I_{\text {FreePeroxide }}\right]} \\
\Delta G=-R T \ln K_{e q}
\end{gathered}
$$

to obtain the unitless equilibrium constant and free energy difference between peroxide complex and aqua complex. The measured and calculated values are listed in the table below.

\begin{tabular}{ccc} 
& $\mathbf{H}_{\mathbf{2}} \mathbf{O}_{\mathbf{2}}$ & $\mathbf{H}_{\mathbf{2}} \mathbf{0}$ \\
\cline { 2 - 3 } $\boldsymbol{S}_{\text {Free }}$ & 9.404 & 2.460 \\
$\mathbf{S}_{\text {Bound }}$ & 9.854 & 6.514 \\
$\boldsymbol{S}_{\text {Exp }}$ & 9.455 & 5.798 \\
$\mathbf{I}_{\text {Exp }}$ & 0.985 & 1.120 \\
$\mathbf{I}_{\text {Bound }}$ & 0.111 & 0.922 \\
IFree & 0.874 & 0.198 \\
\% bound & 0.113 & 0.823 \\
\% free & 0.887 & 0.177
\end{tabular}

Table S2. Calculated values for $1-\mathrm{OH}_{2}$ and $1-\mathrm{H}_{2} \mathrm{O}_{2}$ equilibrium THF-d 8 .

\section{Gravimetric Analysis}

Data collected on a PerkinElmer STA-6000. Samples were loaded into platinum 
crucible and analyzed with the following temperature method: hold at $25^{\circ} \mathrm{C}$ for 1 minute, heat at $5^{\circ} \mathrm{C} / \mathrm{min}$ to $250^{\circ} \mathrm{C}$, hold at $250^{\circ} \mathrm{C}$ for 1 minute.

\section{Preparation of 1- $\mathrm{OH}_{2}$ samples:}

Crystalline $\left[\mathrm{Bu}_{4} \mathrm{~N}\right]\left[\mathrm{Ts}_{3}\right.$ trenZn- $\left.-\mathrm{OH}_{2}\right]$ was crushed to a powder and $42 \mathrm{mg}$ loaded into the crucible. Additionally, $\left[\mathrm{Bu}_{4} \mathrm{~N}\right]\left[\mathrm{Ts}_{3} \operatorname{trenZn}-\mathrm{OH}_{2}\right]$ was dissolved in THF and then precipitated with pentane. After allowing the white solid to fully precipitate, the supernatant was decanted and the solid was dried briefly under vacuum. A portion of this solid was dissolved in $\mathrm{CDCl}_{3}$ and ${ }^{1} \mathrm{H}$ NMR spectroscopy was used to determine the amounts of water and solvent in the solid. A crucible was loaded with $30 \mathrm{mg}$ of this solid for data collection.

\section{Preparation of 1- $\mathrm{H}_{2} \mathrm{O}_{2}$ sample:}

In the glovebox, $\left[\mathrm{Bu}_{4} \mathrm{~N}\right]\left[\mathrm{Ts}_{3}\right.$ trenZn] was dissolved in THF and 1 molar equivalent of hydrogen peroxide solution in THF was added. Pentane was added to this solution to precipitate white solid. After allowing the white solid to full precipitate, the supernatant was decanted and the solid was dried briefly under vacuum. A portion of this solid was dissolved in $\mathrm{CDCl}_{3}$ and ${ }^{1} \mathrm{H}$ NMR spectroscopy was used to determine the relative amounts of water and hydrogen peroxide in the solid. A crucible was loaded with $23 \mathrm{mg}$ of this solid for data collection. 


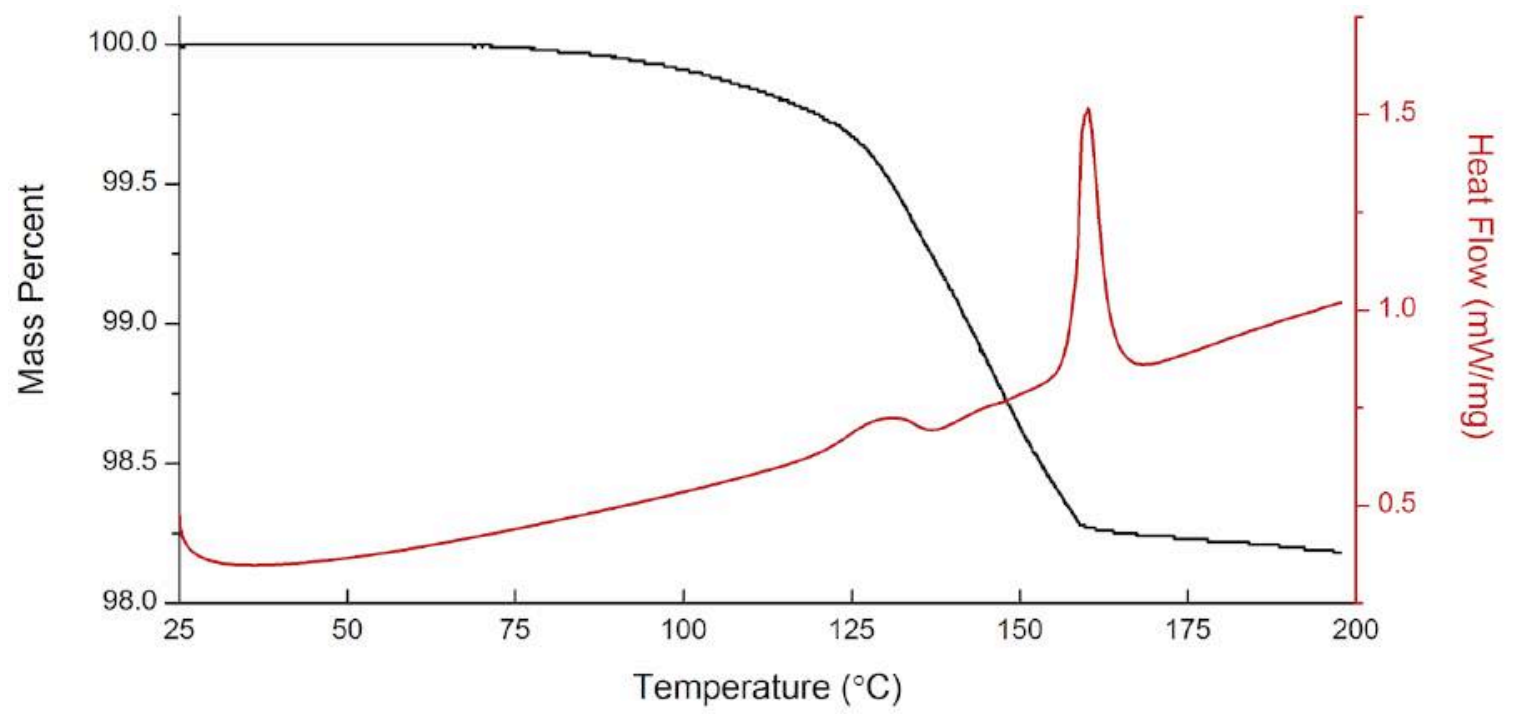

Figure S9. TGA (black) and DSC (red) of crystalline 1- $\mathrm{OH}_{2}$.

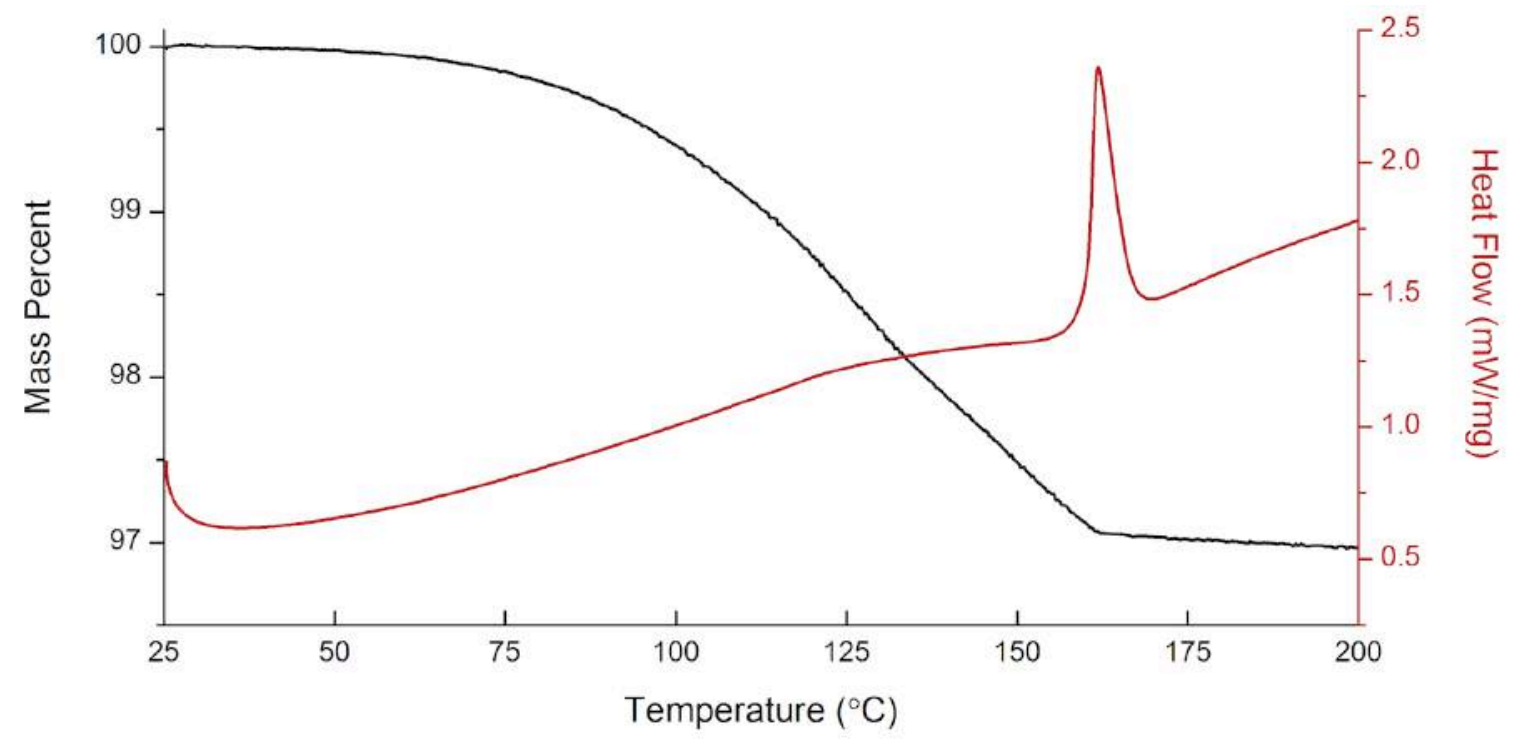

Figure S10. TGA (black) and DSC (red) of precipitated 1-OH 2. 


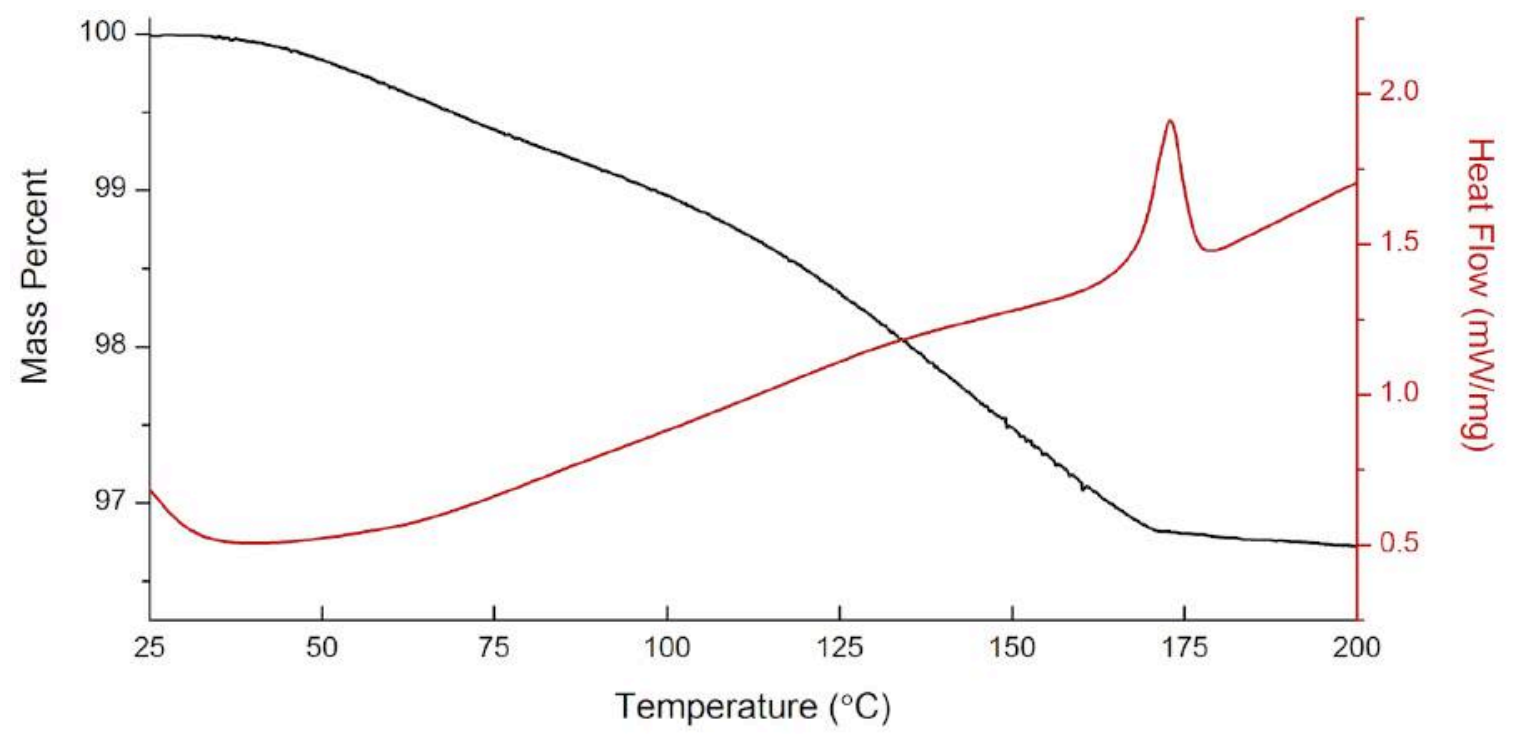

Figure S11. TGA (black) and DSC (red) of precipitated mixture of 1-OHz and 1-

$\mathrm{H}_{2} \mathrm{O}_{2}$. 


\section{NMR Spectra}

\section{Symbol Key}

* - diethyl ether

- DCM

$\dagger$-1,2-dimethoxyethane

A - urea

- - THF

$\star$ - Interference from outside source

\#\# - Residual NMR solvent

$\mathrm{H}_{2} \mathrm{O}_{2}$ (extracted from urea adduct)

${ }^{1} \mathrm{H}$, THF-d $\mathrm{d}_{8} 400 \mathrm{MHz}$

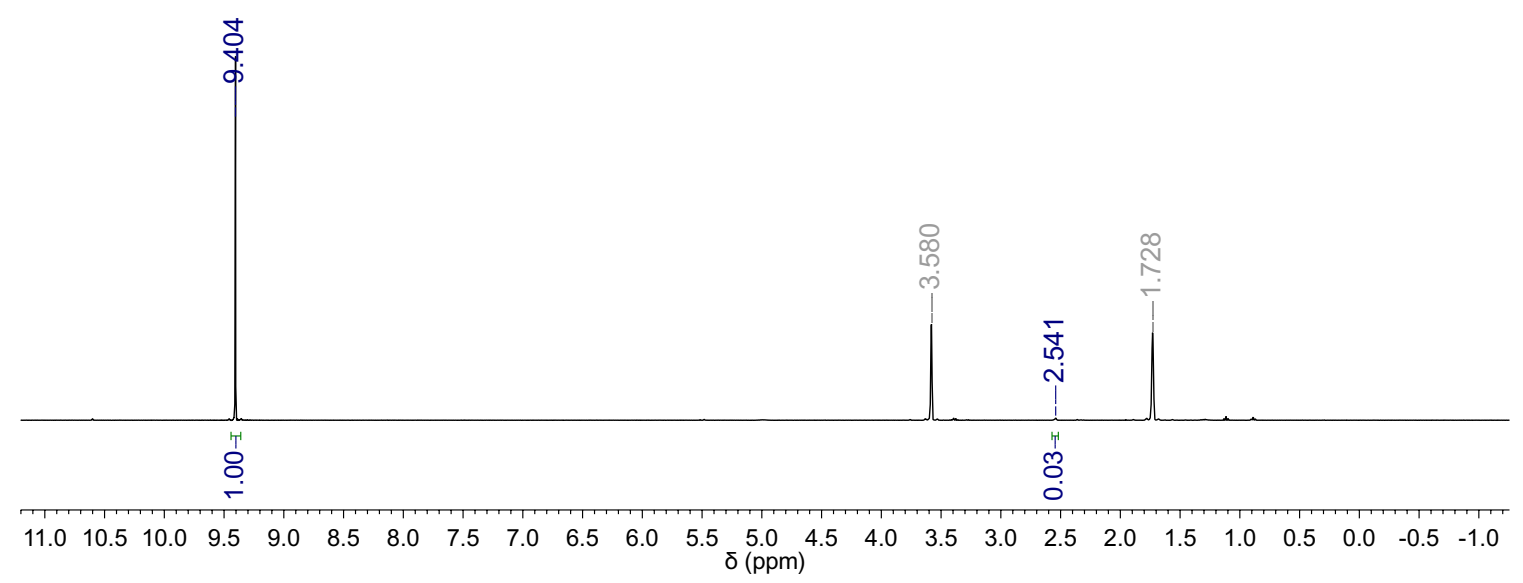

$\mathrm{H}_{3} \mathrm{Ts}_{3}$ tren

${ }_{1} \mathrm{H}, \mathrm{CDCl}_{3} 400 \mathrm{MHz}$

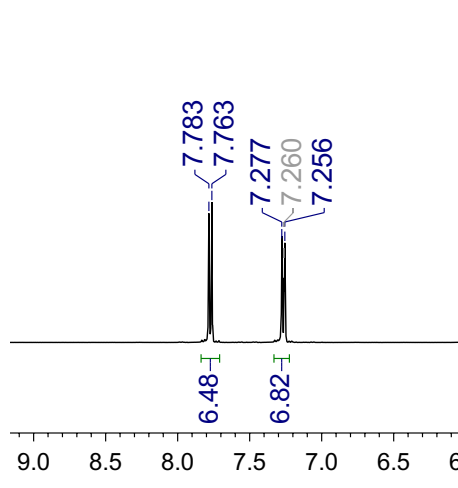

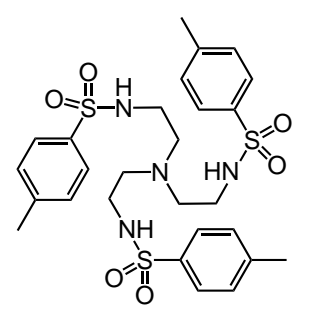

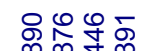

Nivi i i

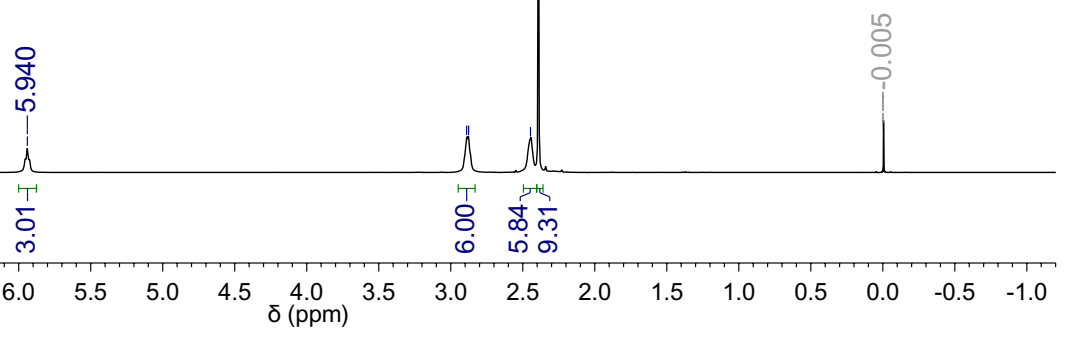



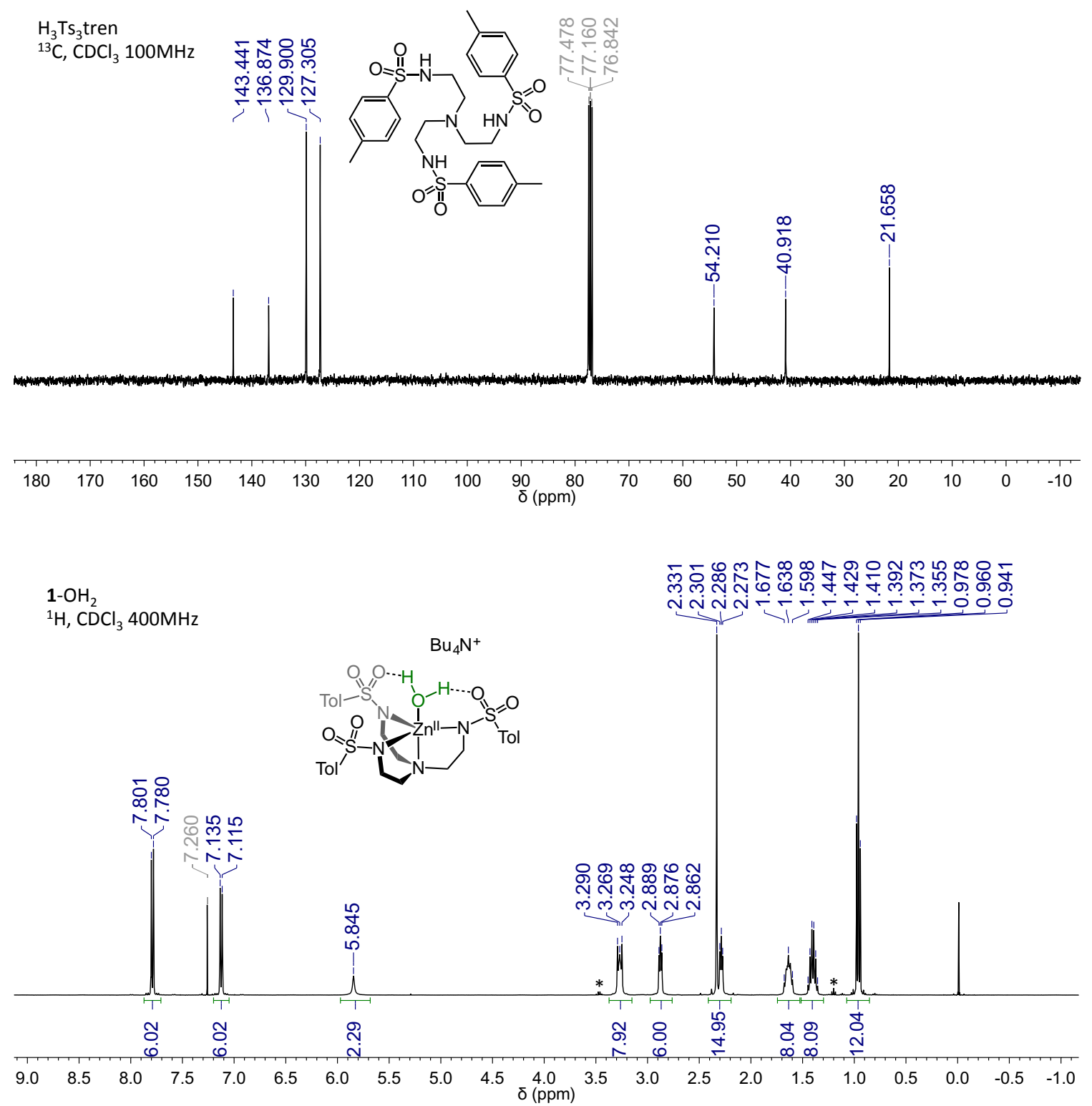

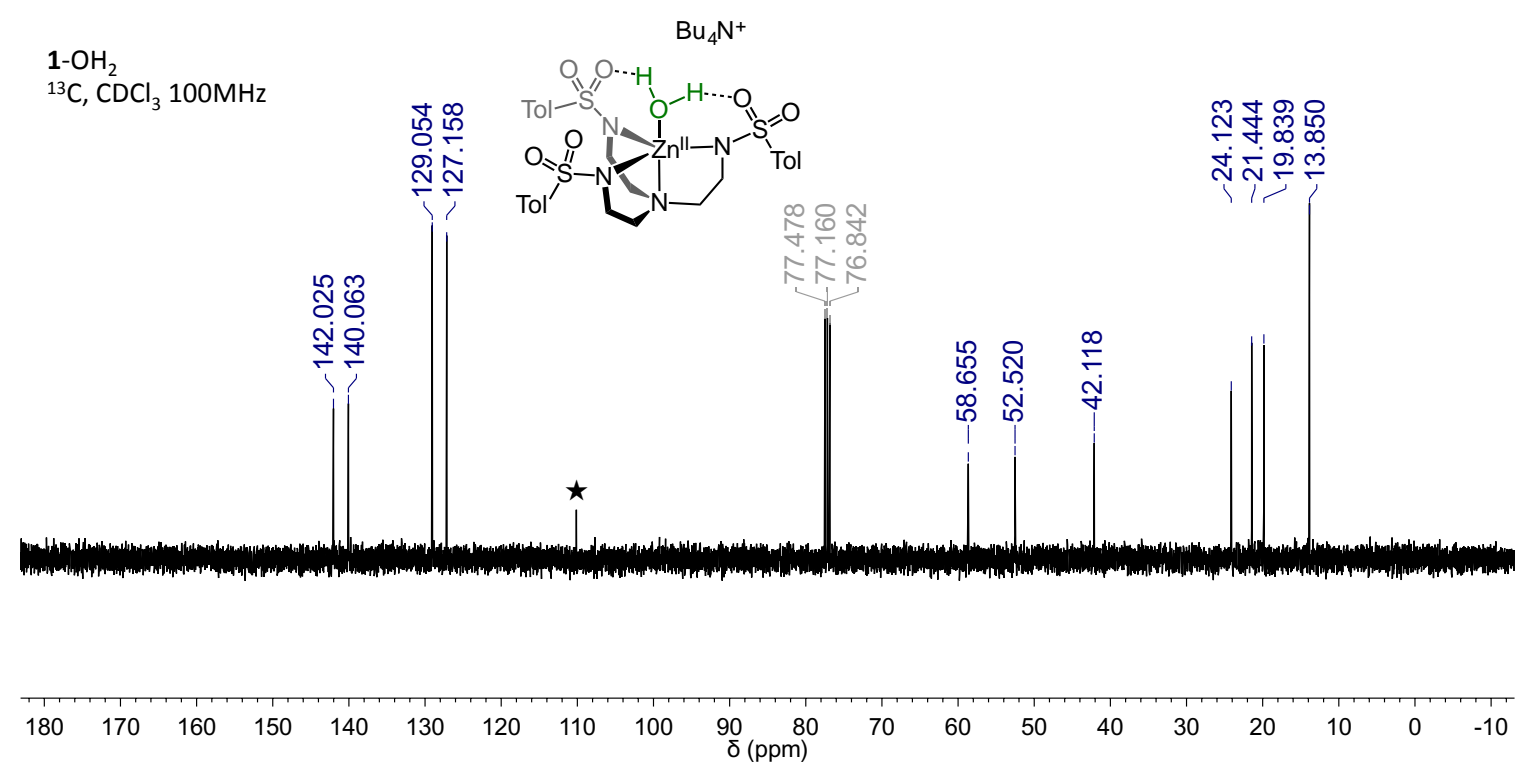

$1-\mathrm{N}_{2} \mathrm{H}_{4}$

${ }^{1} \mathrm{H}, \mathrm{CDCl}_{3} 400 \mathrm{MHz}$
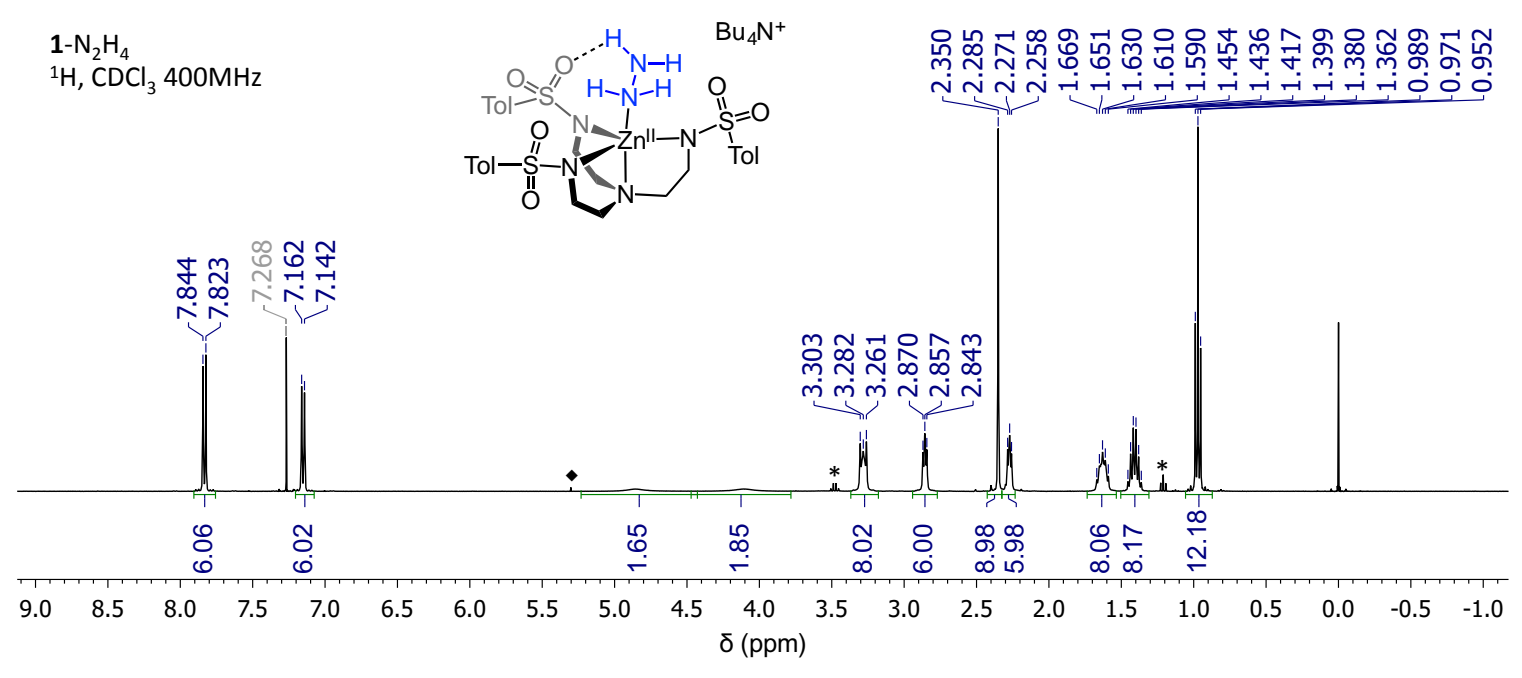

1- $\mathrm{N}_{2} \mathrm{H}_{4}$
${ }^{13} \mathrm{C}, \mathrm{CDCl}_{3} 100 \mathrm{MHz}$
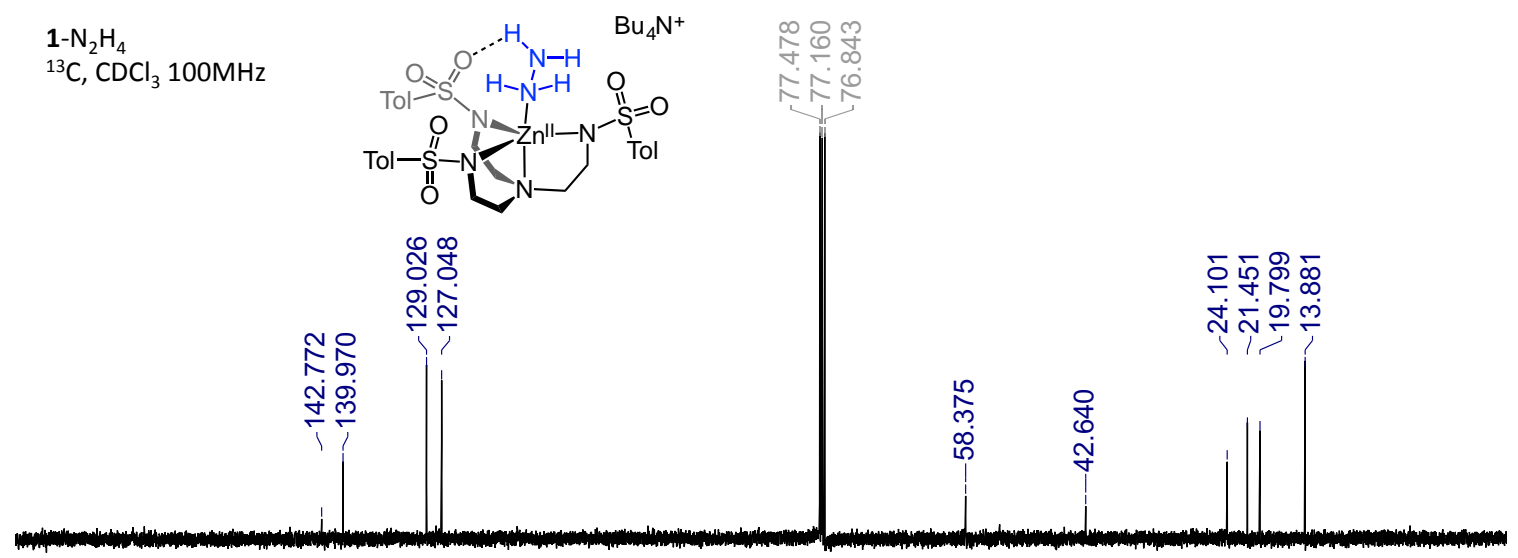

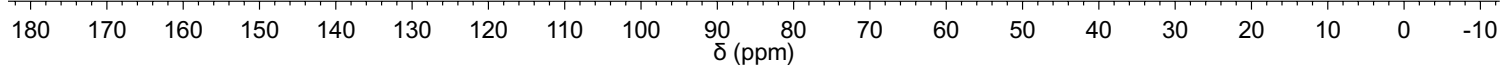



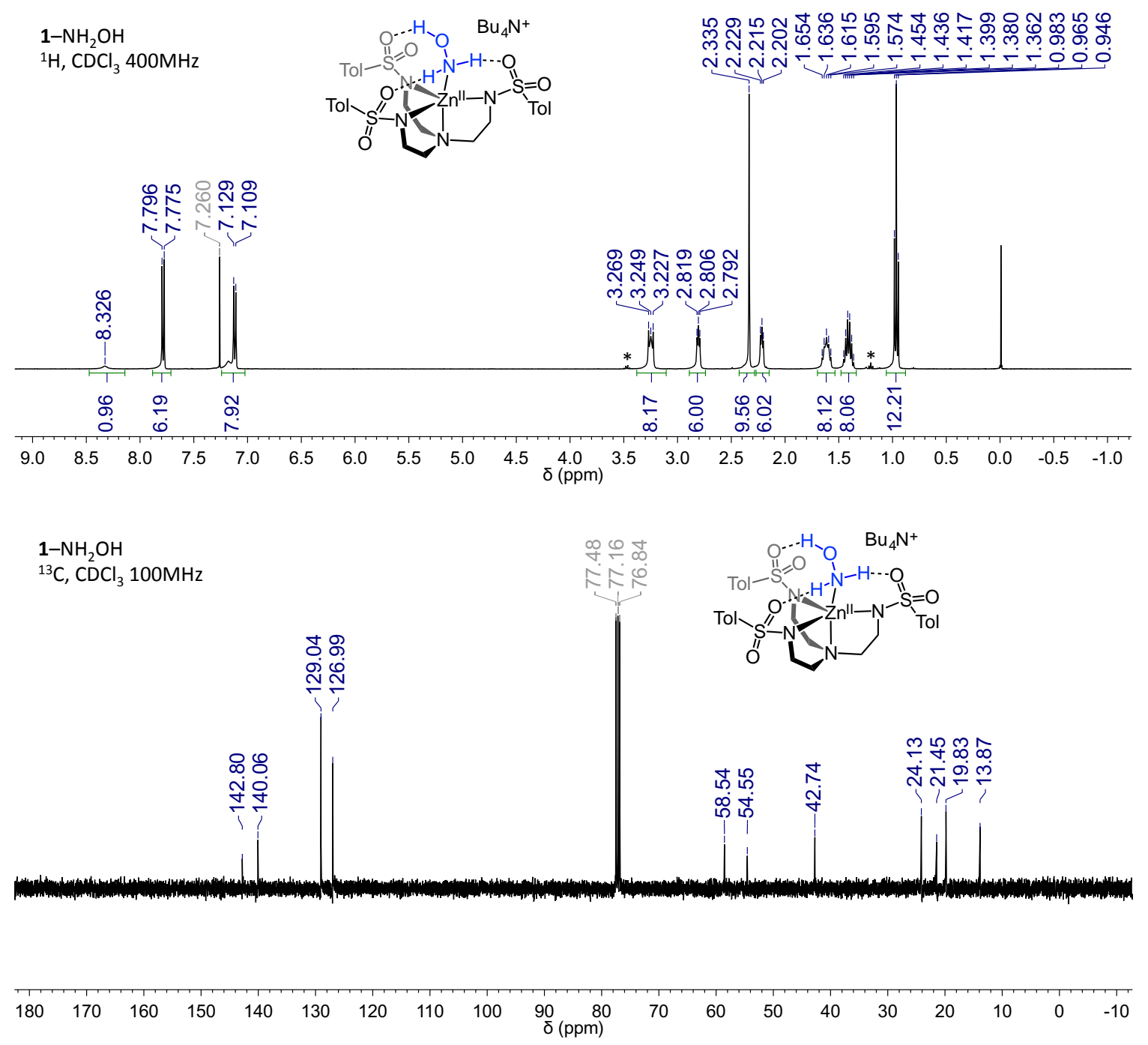

1

${ }^{1} \mathrm{H}, \mathrm{CDCl}_{3} 400 \mathrm{MHz}$

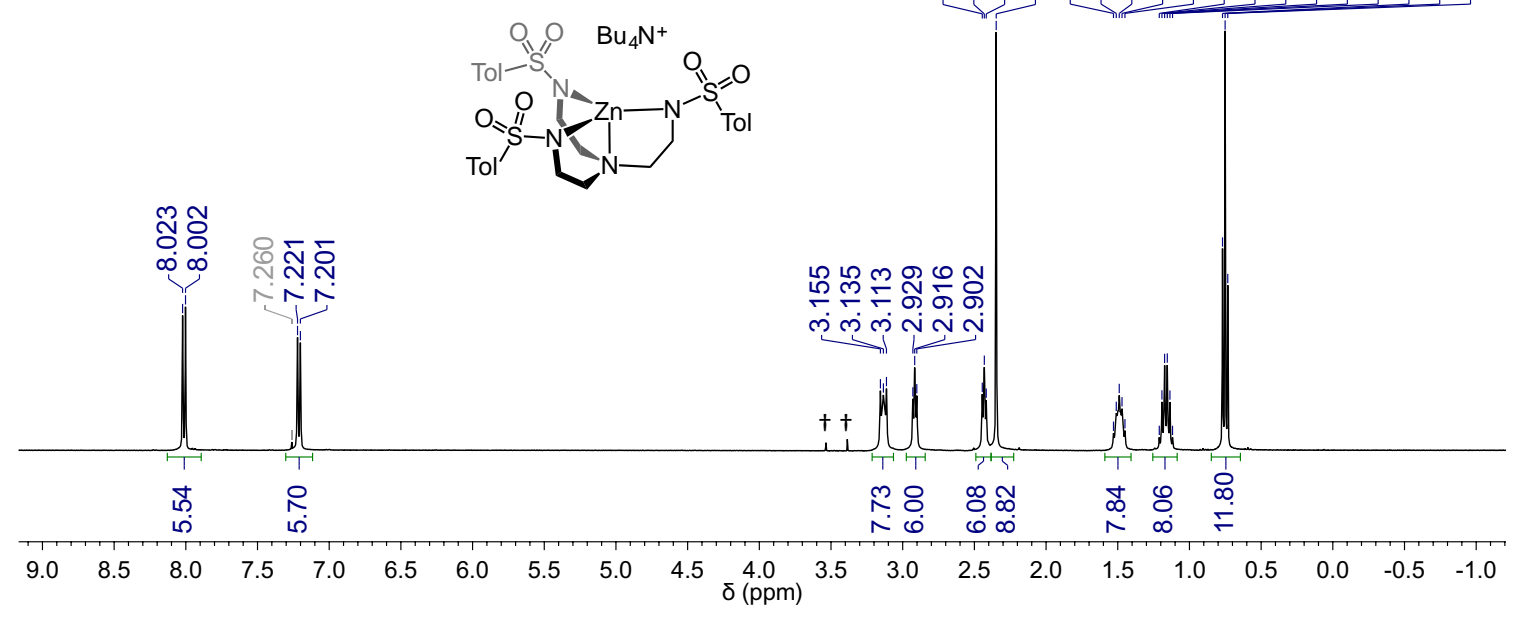




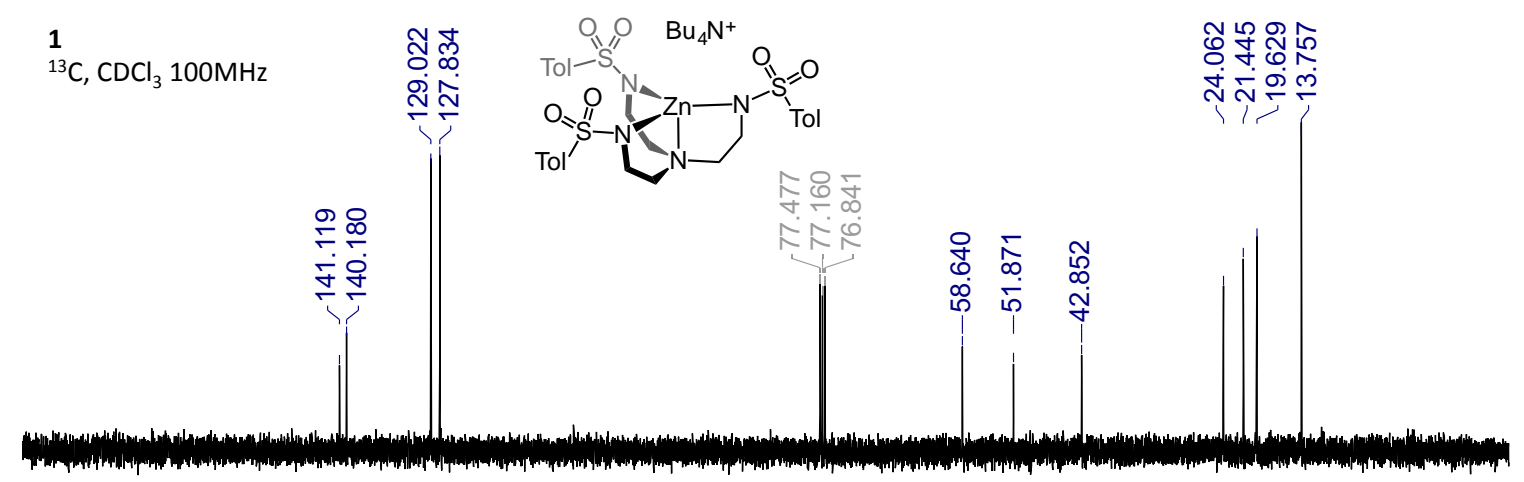

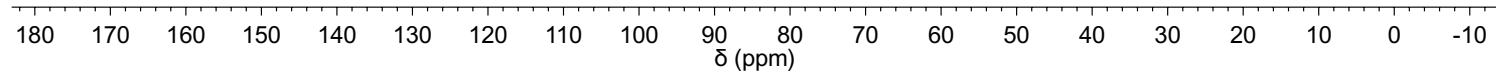

1- $\mathrm{H}_{2} \mathrm{O}_{2}$ ${ }^{1} \mathrm{H}, \mathrm{THF}-\mathrm{d}_{8} 400 \mathrm{MHz}$
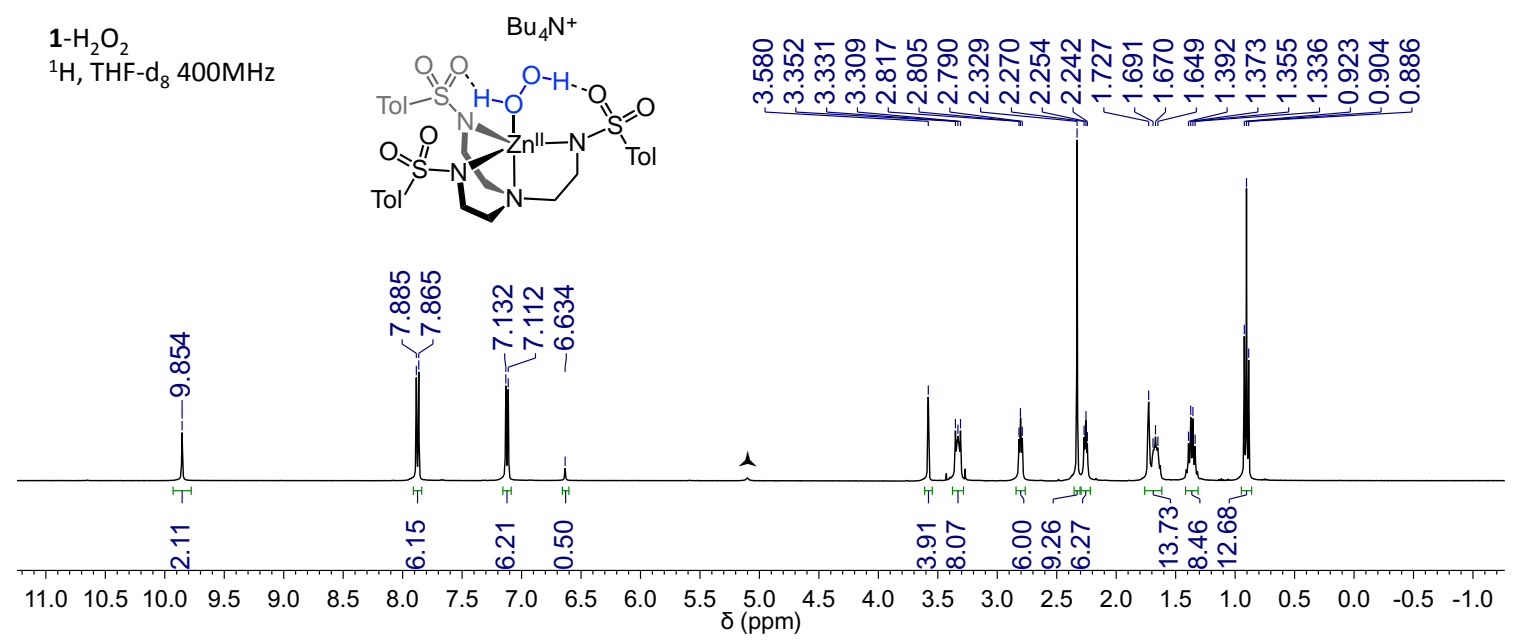

1- $\mathrm{H}_{2} \mathrm{O}_{2} / \mathrm{OH}_{2}$ (crystals)

${ }^{1} \mathrm{H}, \mathrm{CDCl}_{3} 400 \mathrm{MHz}$
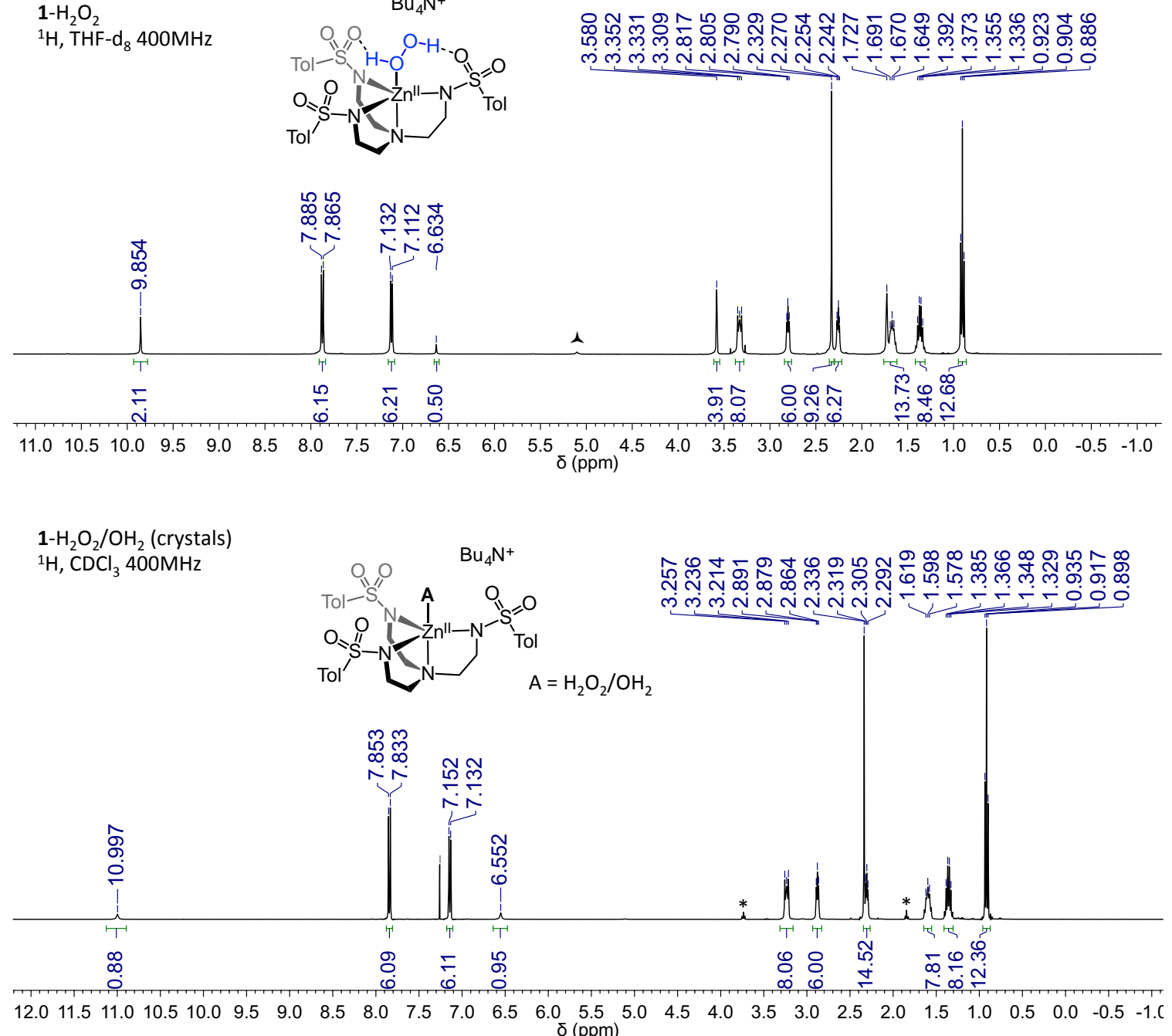

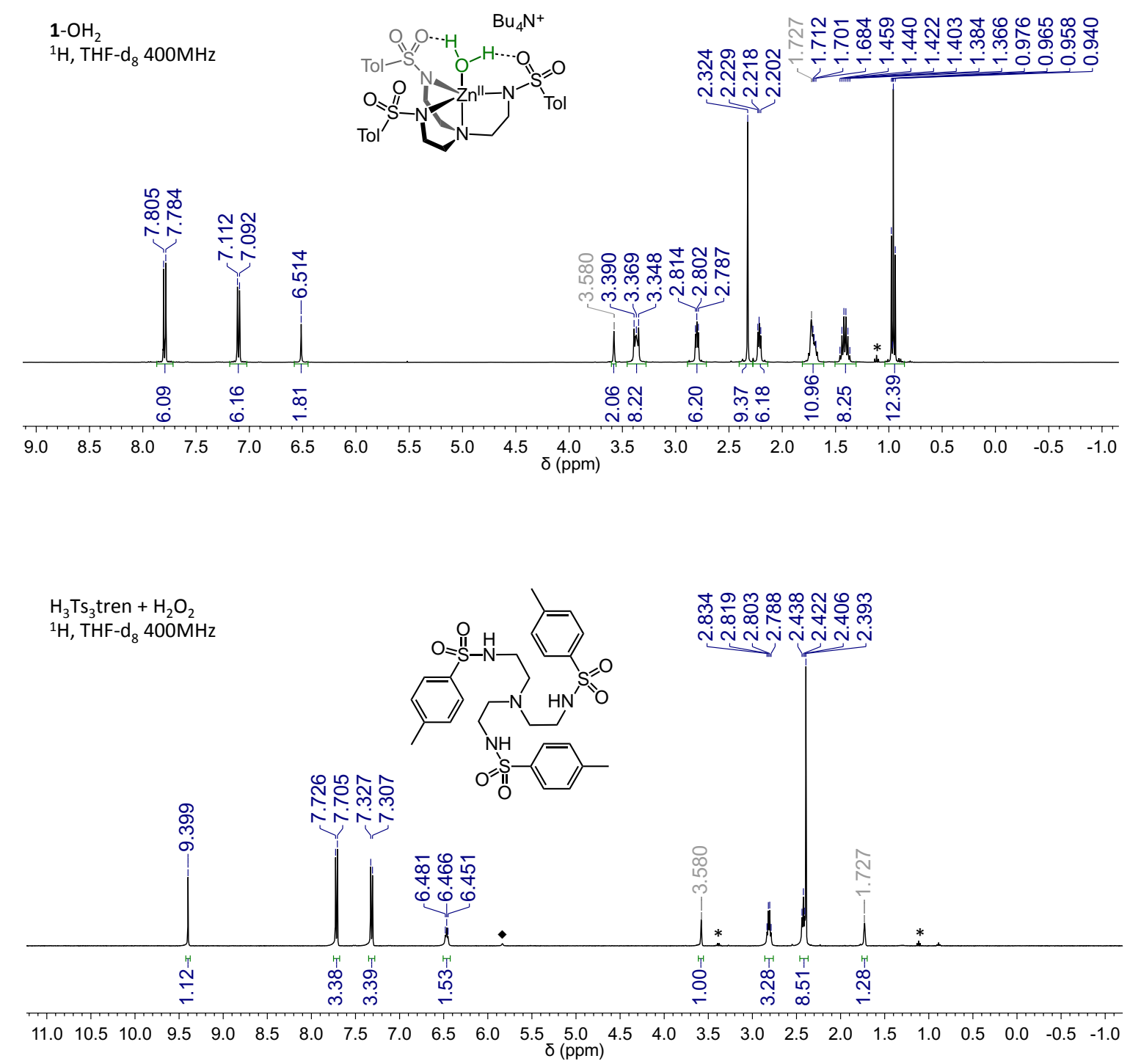

$\mathrm{ZnCl}+\mathrm{H}_{2} \mathrm{O}_{2}$

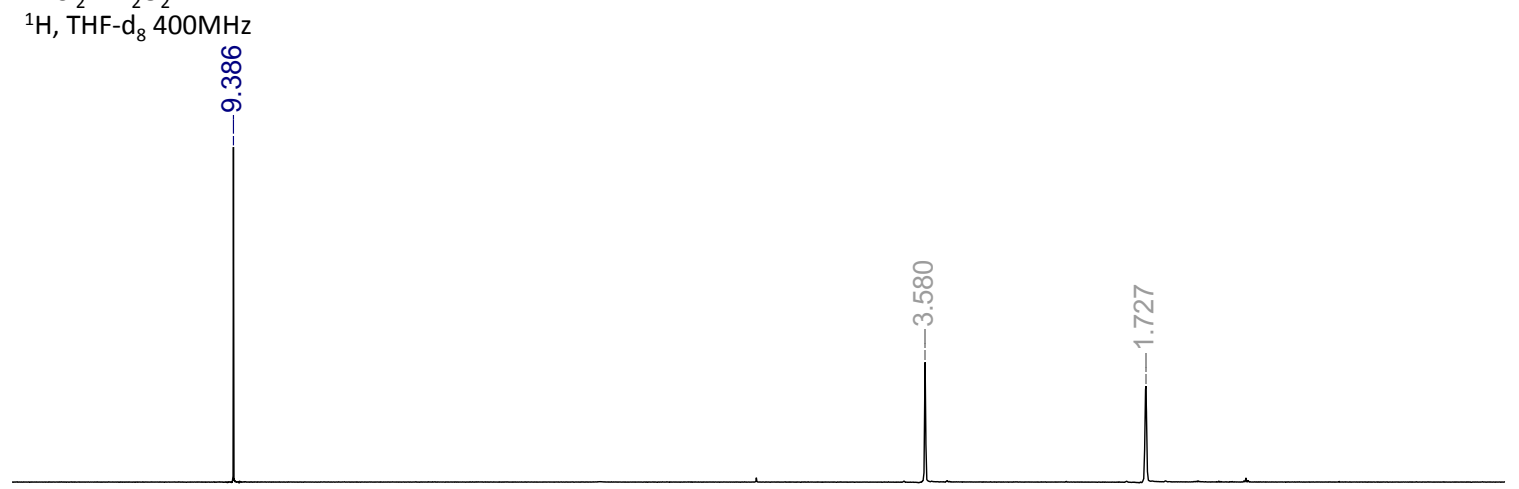

$\begin{array}{llllllllllllllllllllllllll}11.0 & 10.5 & 10.0 & 9.5 & 9.0 & 8.5 & 8.0 & 7.5 & 7.0 & 6.5 & 6.0 & 5.5 & 5.0 & 4.5 & 4.0 & 3.5 & 3.0 & 2.5 & 2.0 & 1.5 & 1.0 & 0.5 & 0.0 & -0.5 & -1.0\end{array}$ 
1- $\mathrm{H}_{2} \mathrm{O}_{2}$ (variable temperature, downfield region) ${ }^{1} \mathrm{H}$, THF- $-\mathrm{d}_{8} 600 \mathrm{MHz}$

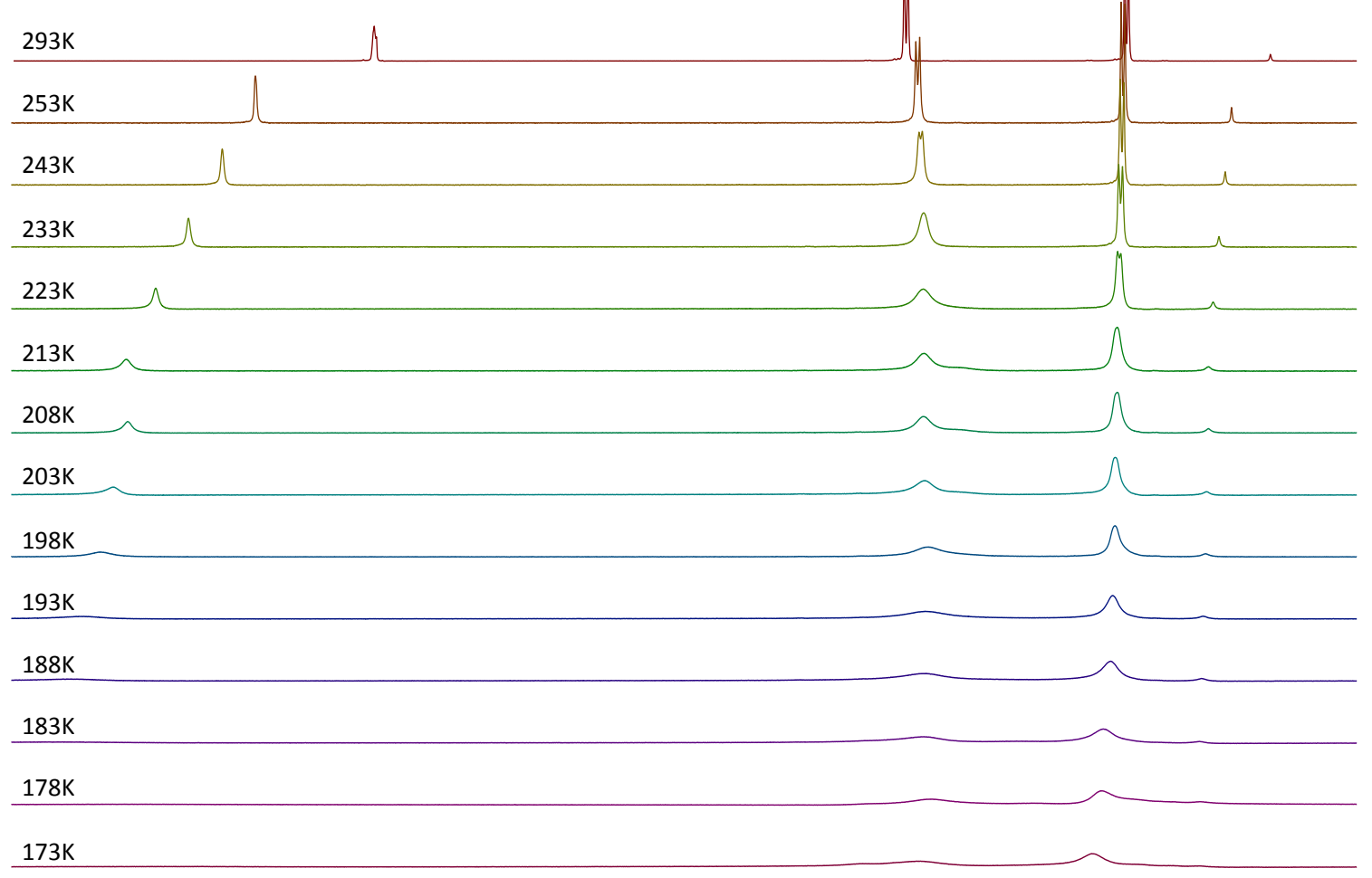

\begin{tabular}{llllllllllllllllllllllllllll}
\hline 1.0 & 10.8 & 10.6 & 10.4 & 10.2 & 10.0 & 9.8 & 9.6 & 9.4 & 9.2 & 9.0 & 8.8 & 8.6 & 8.4 & 8.2 & 8.0 & 7.8 & 7.6 & 7.4 & 7.2 & 7.0 & 6.8 & 6.6 & 6.4
\end{tabular} 
1- $\mathrm{H}_{2} \mathrm{O}_{2}$ (variable temperature, upfield region)

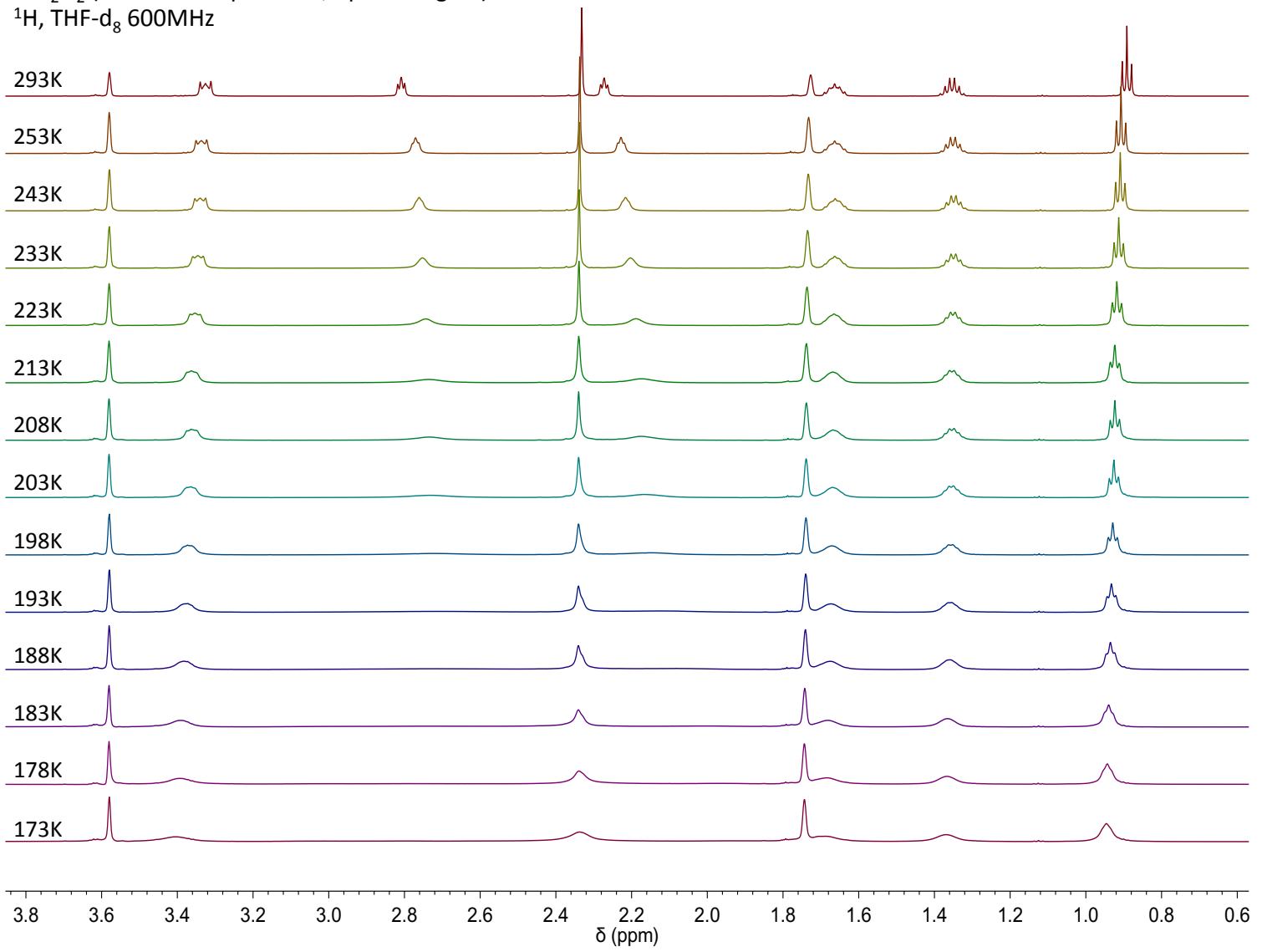




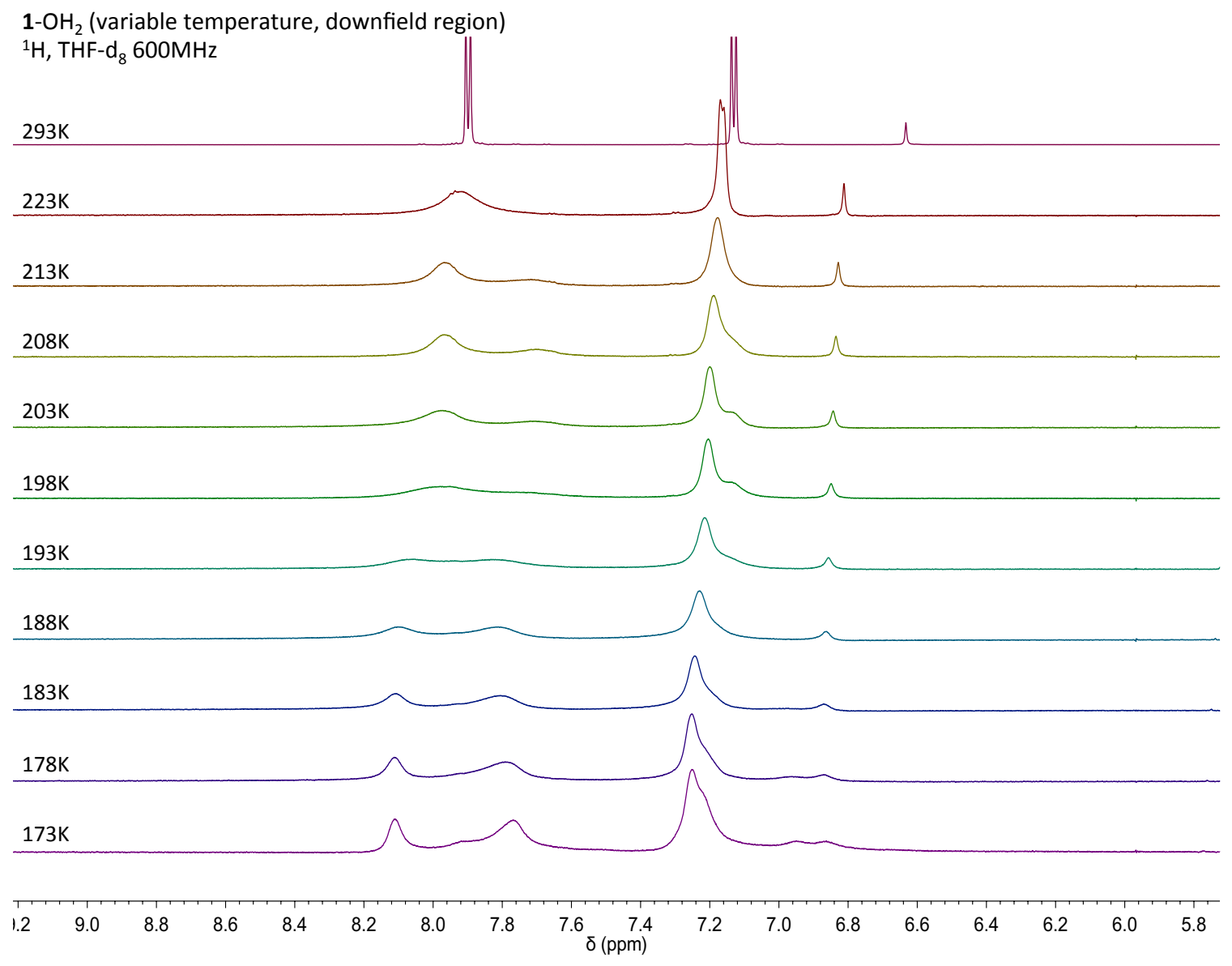


1- $\mathrm{OH}_{2}$ (variable temperature, upfield region)

${ }^{1} \mathrm{H}, \mathrm{THF}-\mathrm{d}_{8} 600 \mathrm{MHz}$

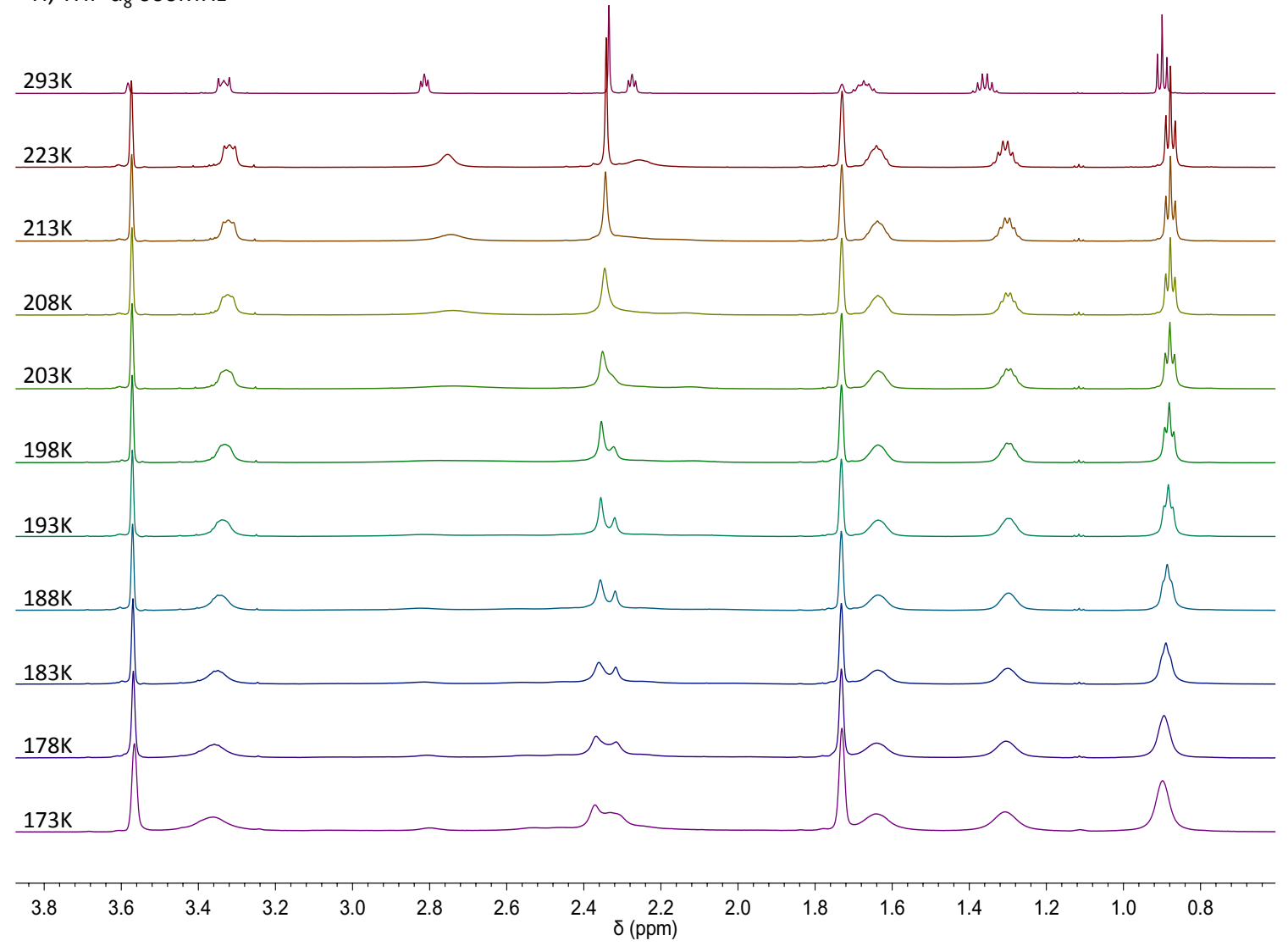

1- $\mathrm{H}_{2} \mathrm{O}_{2} / \mathrm{OH}_{2}$ equilibrium

${ }^{1} \mathrm{H}, \mathrm{THF}-\mathrm{d}_{8} 400 \mathrm{MHz}$
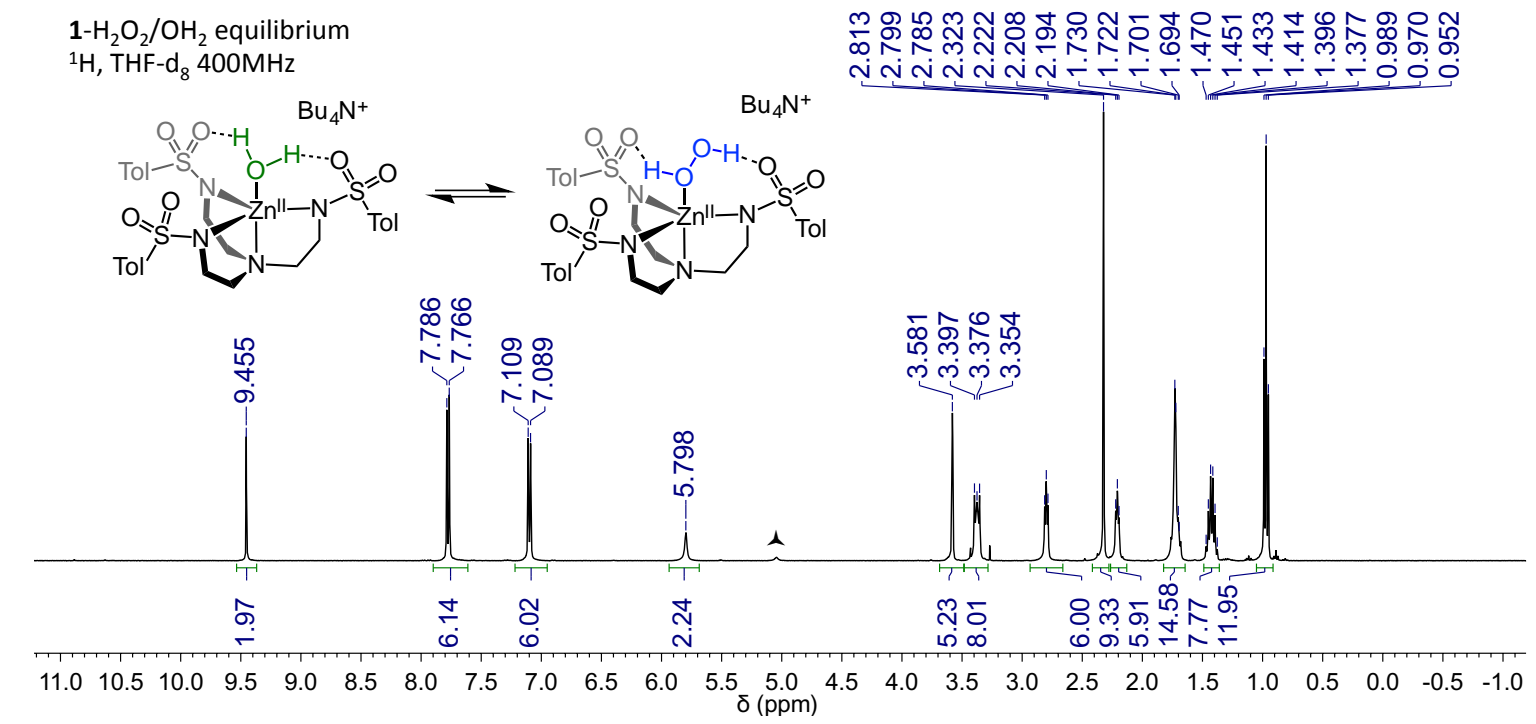

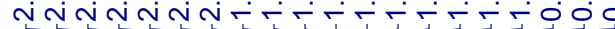


1- $\mathrm{OH}_{2}$ (TGA sample, crushed crystals)

${ }^{1} \mathrm{H}, \mathrm{THF}-\mathrm{d}_{8} 400 \mathrm{MHz}$

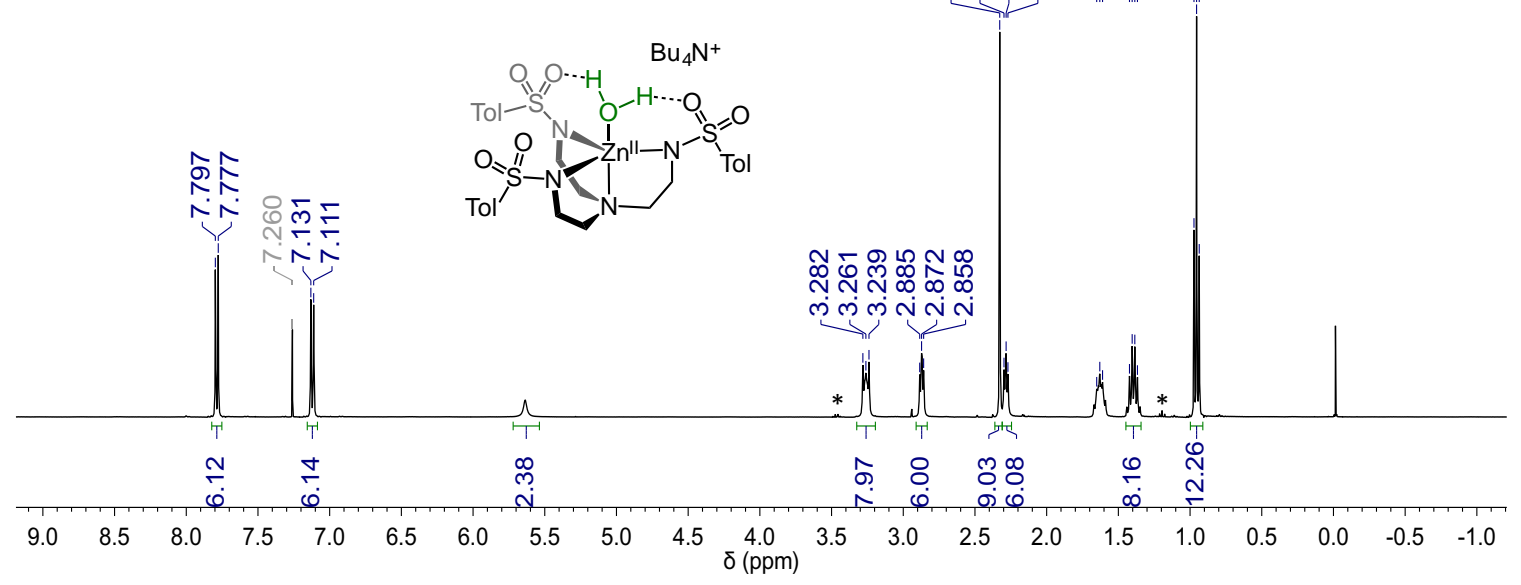

1- $\mathrm{OH}_{2}$ (TGA sample, precipitated material) ${ }^{1} \mathrm{H}, \mathrm{THF}-\mathrm{d}_{8} 400 \mathrm{MHz}$

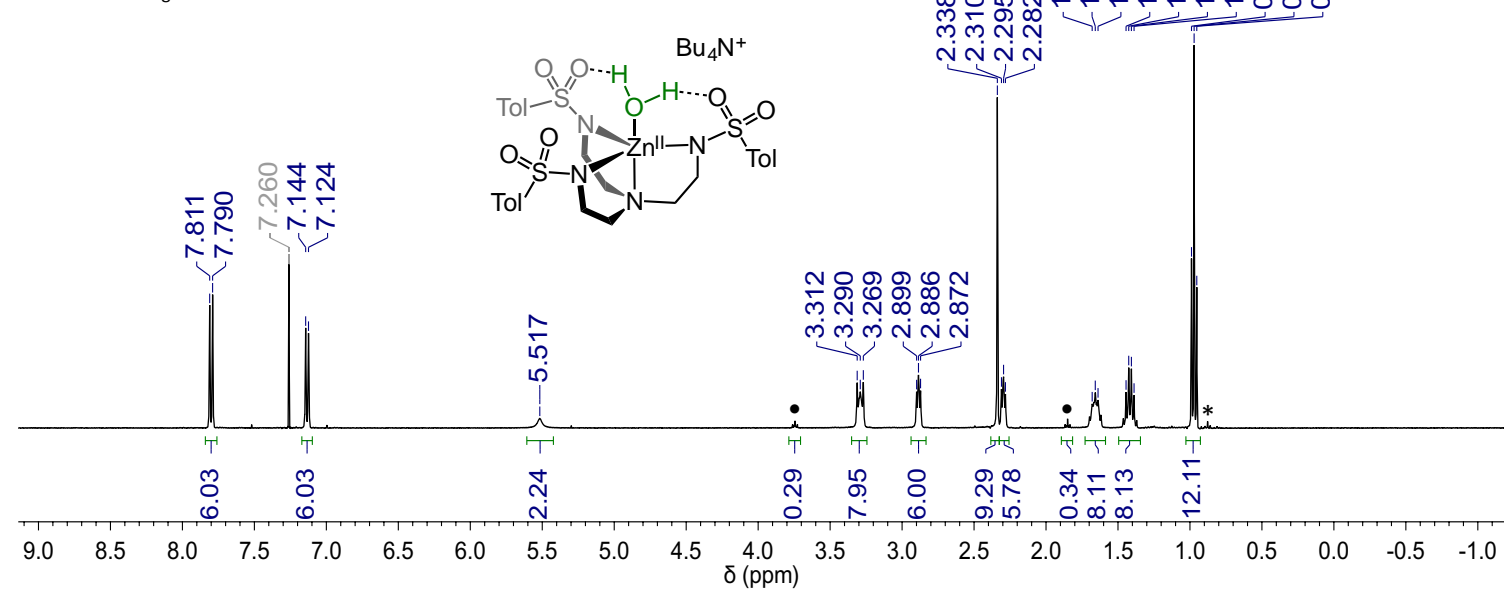

1- $\mathrm{H}_{2} \mathrm{O}_{2}$ (mixture with 1- $\mathrm{OH}_{2}$ )

${ }^{1} \mathrm{H}, \mathrm{THF}-\mathrm{d}_{8} 400 \mathrm{MHz}$
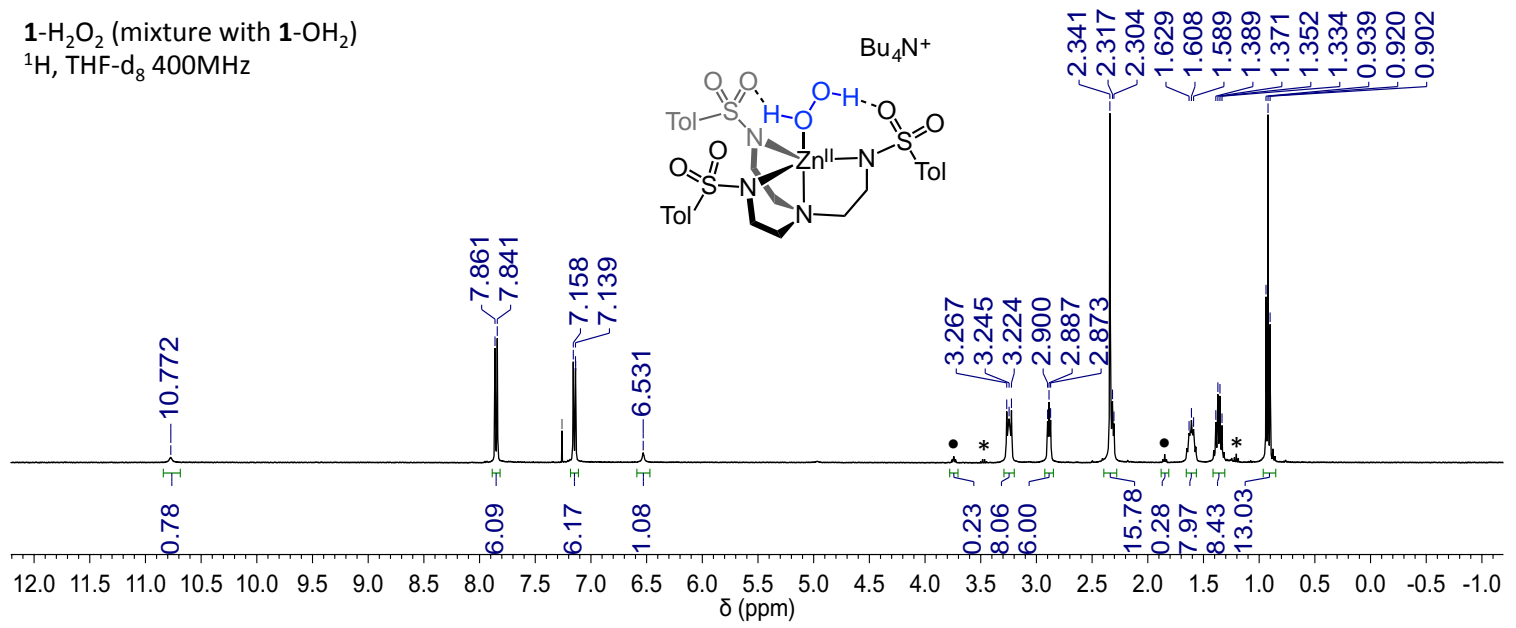


\section{IR Spectra}
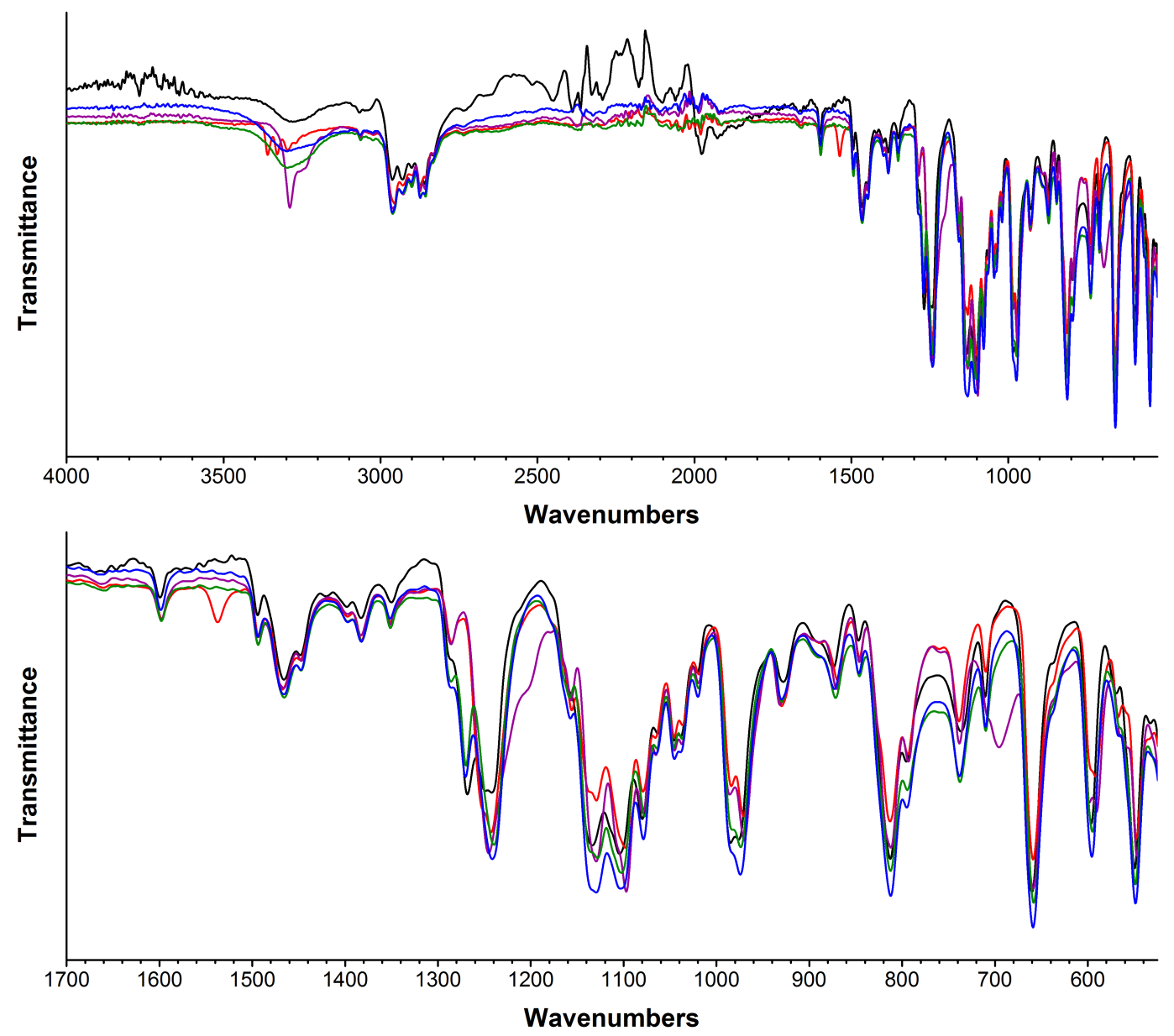

Figure S12. IR Spectra of $1(-), 1-\mathrm{OH}_{2}(-), 1-\mathrm{H}_{2} \mathrm{O}_{2} / 1-\mathrm{OH}_{2}(-)$ (mixed solid), 1 $\mathrm{N}_{2} \mathrm{H}_{4}(-)$, and 1- $\mathrm{NH}_{2} \mathrm{OH}(-)$ (top), with an expansion showing the region where 0-0, $\mathrm{N}-\mathrm{O}$, and $\mathrm{N}-\mathrm{N}$ stretches should be, if observable (bottom). 


\section{Crystallographic Information}

\section{CMW04057BuZnTsAq}
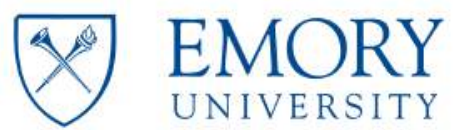

\section{X-ray Crystallography} Center
Submitted by: Christian Wallen

Emory University

Solved by: Marika Wieliczko

Sample ID: $\quad$ CMW 04-057 BuZnTsAq

\section{Crystal Data and Experimental ${ }^{2}$}

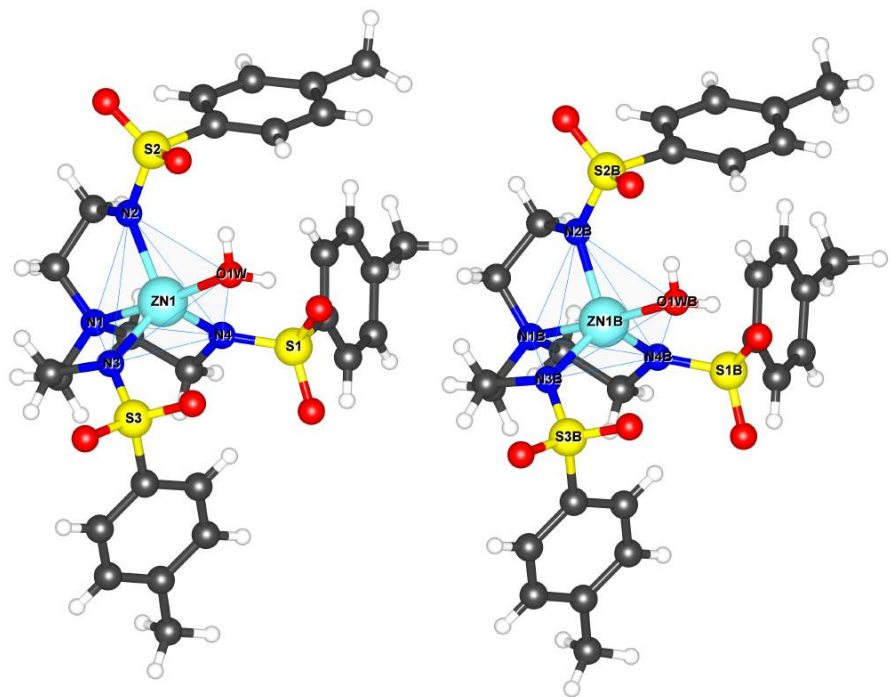

Experimental. Single clear colourless prism-shaped crystals of (CMW04-057BuZnTsAq) were recrystallised from a mixture of DCM and diethyl ether by vapor diffusion. A suitable crystal $(0.59 \times 0.42 \times 0.16)$ was selected and mounted on a loop with paratone oil on a Bruker APEX-II CCD diffractometer. The crystal was cooled to $T=100(2) \mathrm{K}$ during the data collection. The structure was solved with Superflip (L. Palatinus \& G. Chapuis, 2007) using the Charge Flipping algorithm and by using 0lex2 (Dolomanov et al., 2009) as the graphical interface. The model was refined with version of ShelXL97 (Sheldrick, 2008) using Least Squares minimisation.

Crystal Data. $\mathrm{C}_{43} \mathrm{H}_{71} \mathrm{~N}_{5} \mathrm{O}_{7} \mathrm{~S}_{3} \mathrm{Zn}, M_{r}=931.59$, triclinic, P-1 (No. 2), $\quad \mathrm{a}=13.4908(18) \AA, \quad \mathrm{b}=18.694(3) \AA, \quad \mathrm{c}=$ 19.366(3) $\AA, \quad \alpha=98.2422(18)^{\circ}, \quad \beta=92.1817(19)^{\circ}, \quad \gamma=$ 108.3957(18) $, V=4568.7(11) \AA^{3}, T=100(2) \mathrm{K}, Z=4, Z^{\prime}=$
2, $\mu\left(\mathrm{MoK}_{a}\right)=0.728,73265$ reflections measured, 22779 unique $\left(R_{\text {int }}=\right.$ 0.0364) which were used in all calculations. The final $w R_{2}$ was 0.1204 (all data) and $R_{1}$ was 0.0431 (I > 2(I)). 


\begin{tabular}{|c|c|}
\hline Compound & $\begin{array}{l}\text { CMW04-057BuZnT } \\
\text { sAq }\end{array}$ \\
\hline Formula & $\mathrm{C}_{43} \mathrm{H}_{71} \mathrm{~N}_{5} \mathrm{O}_{7} \mathrm{~S}_{3} \mathrm{Zn}$ \\
\hline$D_{\text {calc. }} / \mathrm{g} \mathrm{cm}^{-3}$ & 1.354 \\
\hline$\mu / \mathrm{mm}^{-1}$ & 0.728 \\
\hline Formula Weight & 931.59 \\
\hline Colour & clear colourless \\
\hline Shape & prism \\
\hline Max Size/mm & 0.59 \\
\hline Mid Size/mm & 0.42 \\
\hline Min Size/mm & 0.16 \\
\hline$T / \mathrm{K}$ & $100(2)$ \\
\hline Crystal System & triclinic \\
\hline Space Group & P-1 \\
\hline$a / \AA$ & $13.4908(18)$ \\
\hline$b / \AA$ & $18.694(3)$ \\
\hline$c / \AA$ & $19.366(3)$ \\
\hline$\alpha /^{\circ}$ & $98.2422(18)$ \\
\hline$\beta /^{\circ}$ & $92.1817(19)$ \\
\hline$\gamma /^{\circ}$ & $108.3957(18)$ \\
\hline $\mathrm{V} / \AA^{3}$ & $4568.7(11)$ \\
\hline Z & 4 \\
\hline$Z^{\prime}$ & 2 \\
\hline$\Theta_{\min } /^{\circ}$ & 1.597 \\
\hline$\Theta_{\max } /^{\circ}$ & 28.435 \\
\hline Measured Refl. & 73265 \\
\hline Independent Refl. & 22779 \\
\hline Reflections Used & 17786 \\
\hline$R_{\text {int }}$ & 0.0364 \\
\hline Parameters & 1079 \\
\hline Restraints & 0 \\
\hline Largest Peak & 1.299 \\
\hline Deepest Hole & -0.435 \\
\hline GooF & 1.045 \\
\hline$w R_{2}$ (all data) & 0.1204 \\
\hline$w R_{2}$ & 0.1087 \\
\hline$R_{1}$ (all data) & 0.0588 \\
\hline$R_{1}$ & 0.0431 \\
\hline CCDC Number & 1429660 \\
\hline
\end{tabular}




\section{Structure Quality Indicators}

Refinement:

A clear colourless prism-shaped crystal with dimensions $0.59 \times 0.42 \times 0.16 \mathrm{~mm}-1$ was mounted on a loop with paratone oil. Data were collected using a Bruker APEX-II CCD diffractometer equipped with an Oxford Cryosystems low-temperature apparatus operating at $T=100(2) \mathrm{K}$.

Data were measured using $\phi$ and $\omega$ scans with a narrow frame width using $\mathrm{MoK}_{a}$ radiation (fine-focus sealed tube, $45 \mathrm{kV}, 35 \mathrm{~mA}$ ). The total number of runs and images was based on the strategy calculation from the program APEXII (Bruker, 2014). The maximum resolution achieved was $\Theta=28.43^{\circ}$.

Unit cell indexing was performed by using the APEXII (Bruker, 2014) software and refined using SAINT (Bruker, V8.34A, 2013) on 9792 reflections, 13\% of the observed reflections. Data reduction, scaling and absorption corrections were performed using SAINT (Bruker, V8.34A, 2013) and SADABS-2014/5 (Bruker,2014) (for scaling/absorption correction), $w R_{2}$ (int) was 0.1135 before and 0.0459 after correction. The ratio of minimum to maximum transmission is 0.8348 . The $\lambda / 2$ correction factor is 0.00150 .. The final completeness is 100.00 out to 28.435 in $\Theta$. The absorption coefficient $(\mu)$ of this material is $0.728 \mathrm{~mm}^{-1}$ and the minimum and maximum transmissions are 0.6225 and 0.7457 .

The structure was solved with Superflip (L. Palatinus \& G. Chapuis, 2007) using the Charge Flipping solution method and by using 0lex2 (Dolomanov et al., 2009) as the graphical interface. The structure was refined by Least Squares using version of ShelXL-97 (Sheldrick, 2008). All non-hydrogen atoms were refined anisotropically. Hydrogen atom positions were calculated geometrically and refined using the riding model.

The value of $Z^{\prime}$ is 2 . This means that there are two independent molecules in the asymmetric unit. 


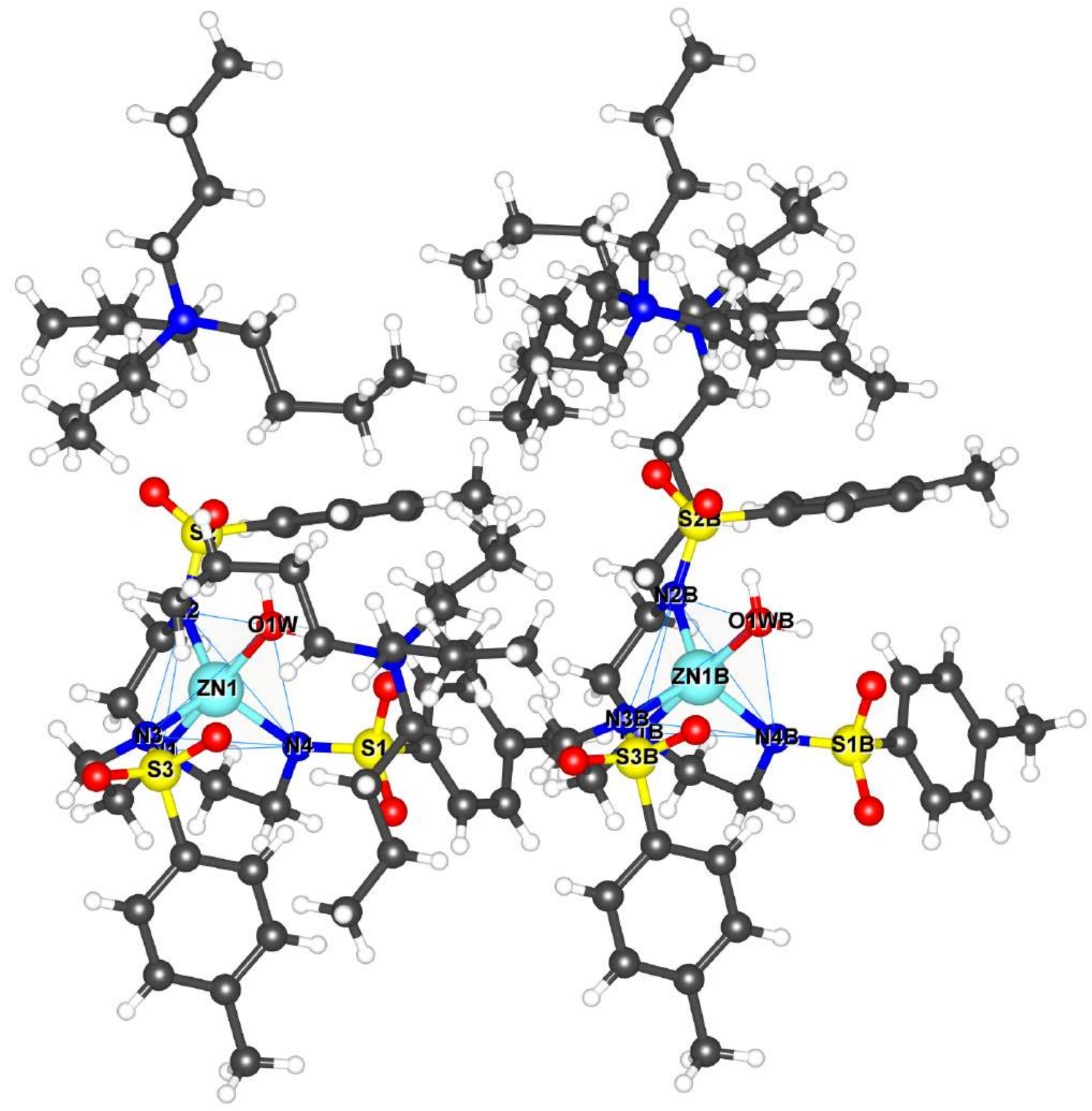

Figure 1: Plot of the asymmetric unit 


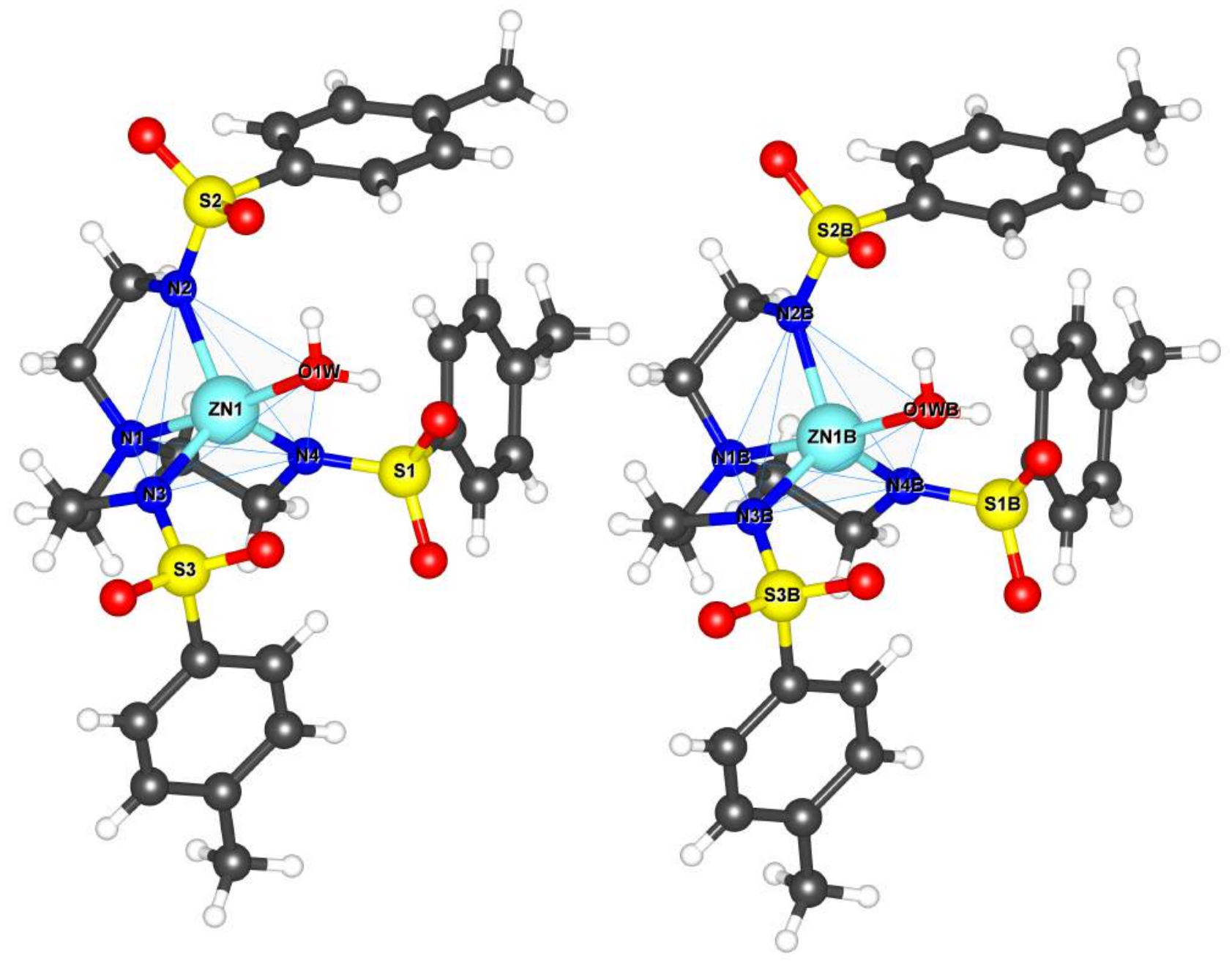

Figure 2: The two independent ZnLAq molecules.

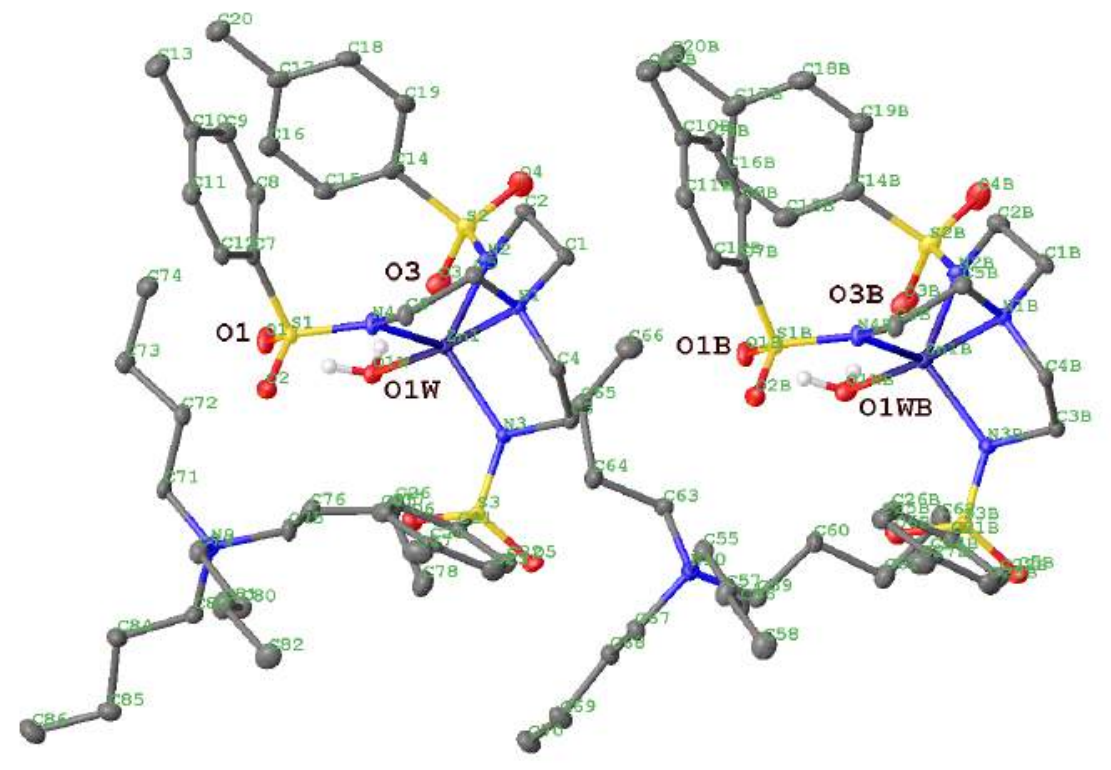

Figure 3: The following hydrogen bonding interactions with a maximum D-D distance of $2.9 \AA$ and a minimum angle of $120^{\circ}$ are present in CMW04_057BuZnTsAq: 01W-01 $=2.695 \AA, 01 W-03=2.712 \AA, 01 W B-01 \mathrm{~B}=2.689 \AA$, 01WB-03B = 2.731 A. 


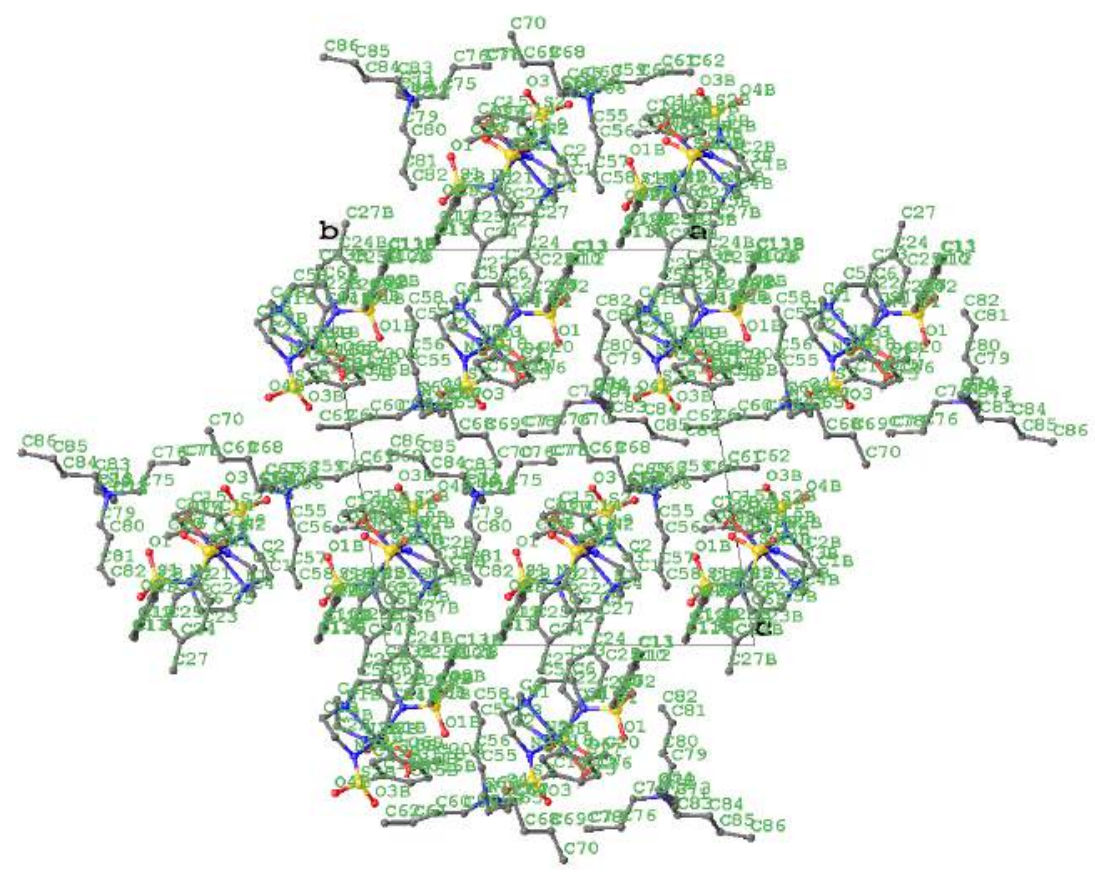

Figure 4: Packing diagram of CMW04_057BuZnTsAq.

\section{Data Plots: Diffraction Data}
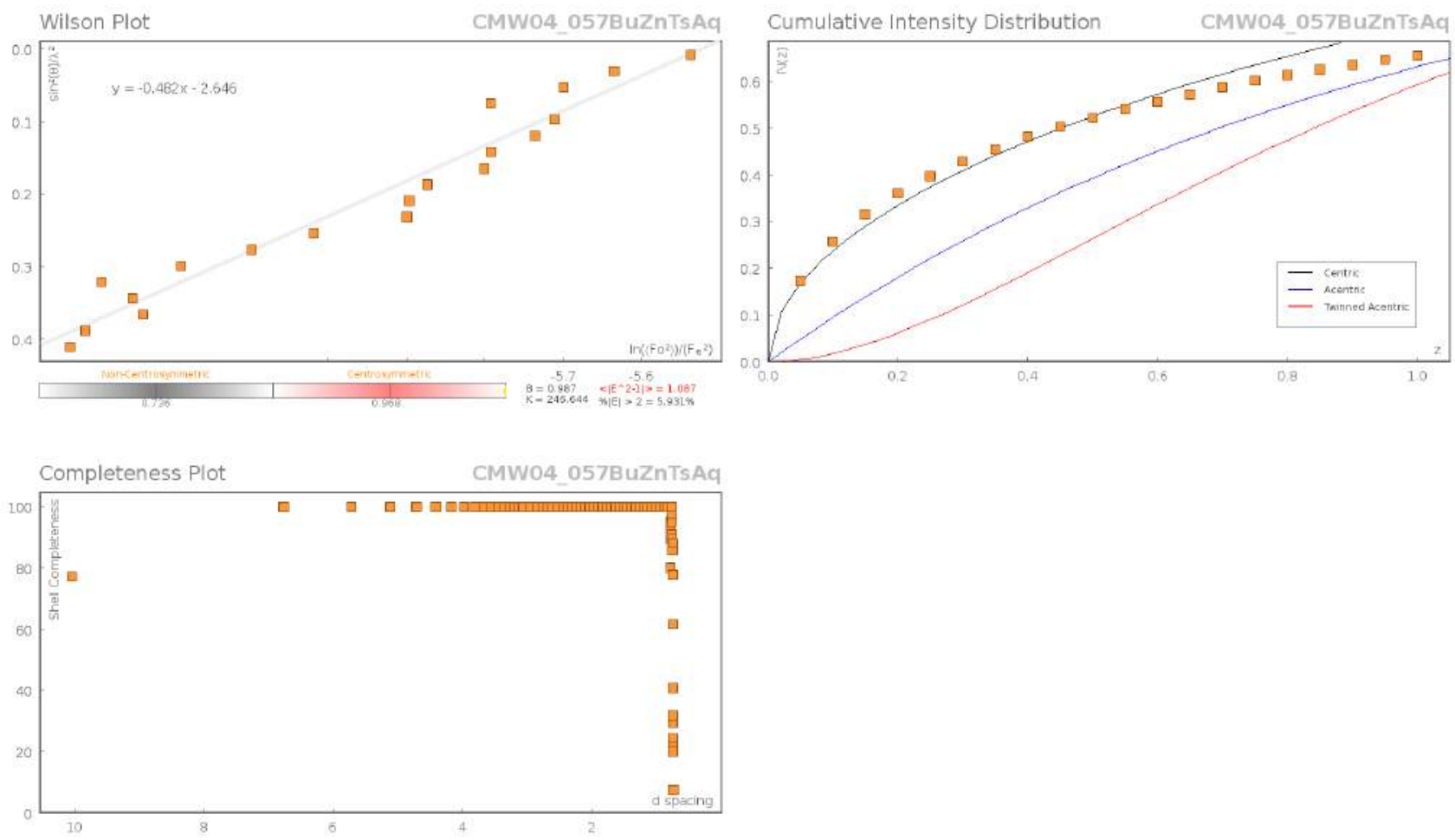
Data Plots: Refinement and Data
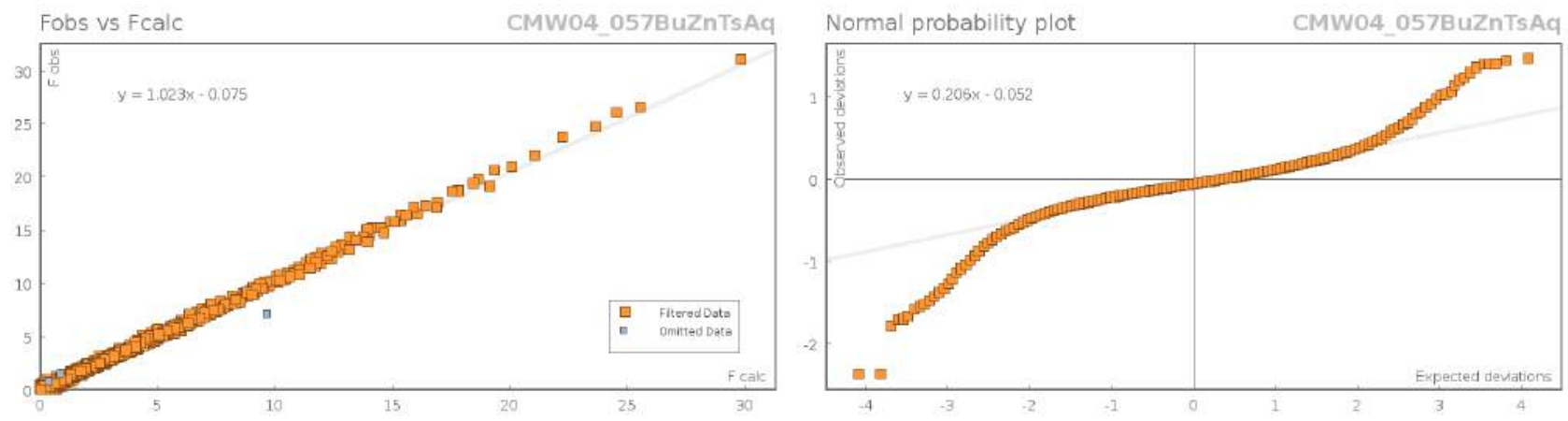

\section{Reflection Statistics}

\begin{tabular}{|c|c|c|c|}
\hline $\begin{array}{l}\text { Total reflections (after } \\
\text { filtering) }\end{array}$ & 73274 & Unique reflections & 22779 \\
\hline Completeness & 0.99 & Mean $\mathrm{I} / \sigma$ & 15.05 \\
\hline hklsub $>\max </$ sub $>$ collected & $(18,24,25)$ & hklsub $>\min </$ sub $>$ collected & $(-18,-24,-25)$ \\
\hline hklmax used & $(18,24,25)$ & hklmin used & $(-18,-24,0)$ \\
\hline Lim $d_{\max }$ collected & 100.0 & Lim $d_{\min }$ collected & 0.36 \\
\hline$d_{\max }$ used & 12.75 & $\mathrm{~d}_{\min }$ used & 0.75 \\
\hline Friedel pairs & 17513 & Friedel pairs merged & 1 \\
\hline Inconsistent equivalents & 0 & $\mathrm{R}_{\text {int }}$ & 0.0364 \\
\hline $\mathrm{R}_{\text {sigma }}$ & 0.0398 & Intensity transformed & 0 \\
\hline Omitted reflections & 0 & Omitted by user (OMIT hkl) & 9 \\
\hline Multiplicity & $(15940,16863,6360,1132)$ & Maximum mulitplicity & 8 \\
\hline Removed systematic absences & 0 & Filtered off (Shel/OMIT) & 0 \\
\hline
\end{tabular}

\section{Images of the Crystal on the Diffractometer}

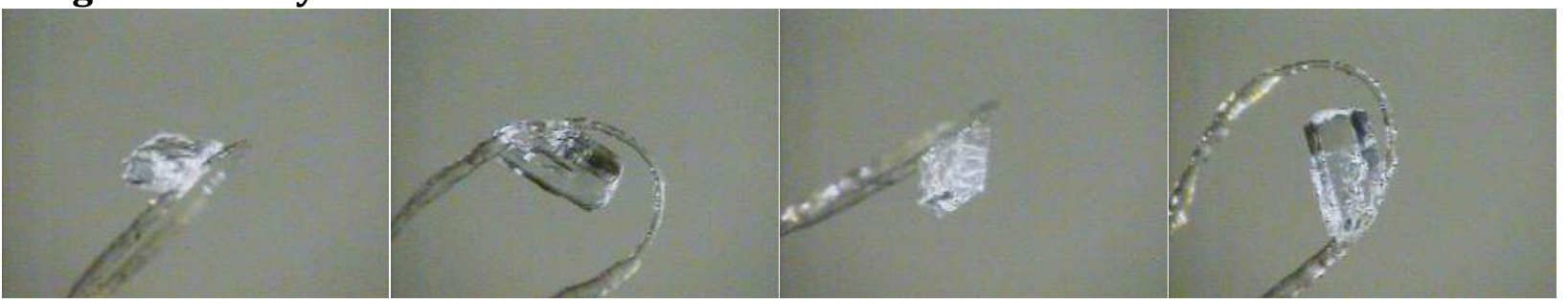

Table 1: Fractional Atomic Coordinates $\left(\times 10^{4}\right)$ and Equivalent Isotropic Displacement Parameters $\left(\AA^{2} \times 10^{3}\right)$ for CMW04_057BuZnTsAq. $U_{e q}$ is defined as $1 / 3$ of the trace of the orthogonalised $U_{i j}$.

\begin{tabular}{lrrrr}
\hline Atom & \multicolumn{1}{c}{$\mathbf{x}$} & $\mathbf{y}$ & $\mathbf{z}$ & $\boldsymbol{U}_{\boldsymbol{e q}}$ \\
\hline Zn1 & $297.5(2)$ & $5781.6(2)$ & $2363.5(2)$ & $9.43(6)$ \\
Zn1B & $284.6(2)$ & $10645.2(2)$ & $2378.3(2)$ & $10.66(6)$ \\
S3 & $-2173.2(3)$ & $5587.7(3)$ & $2386.0(2)$ & $10.12(9)$ \\
S1 & $185.0(4)$ & $4050.4(3)$ & $1613.0(2)$ & $13.3(1)$ \\
S1B & $185.2(4)$ & $8962.3(3)$ & $1521.7(2)$ & $12.78(10)$ \\
S3B & $-2152.4(3)$ & $10532.3(3)$ & $2398.5(2)$ & $12.65(10)$ \\
S2 & $2435.6(4)$ & $6644.8(3)$ & $3436.5(2)$ & $13.84(10)$ \\
S2B & $2381.6(4)$ & $11347.5(3)$ & $3483.7(2)$ & $15.92(10)$ \\
01B & $265.3(11)$ & $8802.2(8)$ & $2230.3(7)$ & $19.2(3)$ \\
01 & $319.2(12)$ & $3960.8(8)$ & $2343.0(7)$ & $21.1(3)$ \\
05B & $-2787.4(11)$ & $10999.8(8)$ & $2643.7(8)$ & $18.9(3)$ \\
02B & $-739.8(11)$ & $8473.9(8)$ & $1077.9(8)$ & $19.7(3)$ \\
05 & $-2827.1(10)$ & $6054.2(8)$ & $2598.0(7)$ & $14.9(3)$ \\
03 & $1756.6(11)$ & $6316.9(9)$ & $3957.1(7)$ & $18.4(3)$
\end{tabular}




\begin{tabular}{|c|c|c|c|c|}
\hline Atom & $\mathbf{x}$ & $\mathbf{y}$ & $\mathbf{z}$ & $U_{e q}$ \\
\hline 02 & $-767.8(11)$ & $3535.3(8)$ & $1219.9(8)$ & $21.6(3)$ \\
\hline o1W & $143.4(10)$ & 5169.8(8) & $3209.3(7)$ & 15.1(3) \\
\hline O1WB & $43.0(11)$ & $9945.4(9)$ & $3158.0(7)$ & $17.6(3)$ \\
\hline 04 & 3182.1(11) & 7399.4(9) & $3662.2(8)$ & $22.8(3)$ \\
\hline O3B & $1657.3(11)$ & $11034.9(9)$ & $3985.9(7)$ & $21.5(3)$ \\
\hline 06 & $-2176(1)$ & 4997.3(8) & $2788.6(7)$ & $15.8(3)$ \\
\hline O6B & $-2200.3(11)$ & $9892.8(8)$ & $2748.4(7)$ & 18.3(3) \\
\hline O4B & $3196.4(12)$ & 12064.4(9) & $3733.9(8)$ & $24.4(3)$ \\
\hline N4 & 368.8(13) & 4919.3(9) & 1595.7(8) & $12.8(3)$ \\
\hline N4B & $365.1(12)$ & 9837.8(9) & $1562.8(8)$ & $12.6(3)$ \\
\hline N3B & $-967.6(12)$ & $11023.7(9)$ & 2388.6(8) & $12.1(3)$ \\
\hline N2B & $1725.7(12)$ & $11379.8(10)$ & $2810.1(8)$ & $14.3(3)$ \\
\hline N2 & $1727.2(12)$ & 6590.4(9) & 2758.8(8) & $12.6(3)$ \\
\hline N1 & $627.1(11)$ & $6432.9(9)$ & $1471.3(8)$ & $9.0(3)$ \\
\hline N1B & 692.2(12) & 11380.1(9) & $1534.6(8)$ & $10.6(3)$ \\
\hline N3 & $-1007.0(11)$ & 6095.2(9) & 2338.2(8) & 10.1(3) \\
\hline N10 & $-2472.3(12)$ & 7959.7(9) & $3869.7(8)$ & $12.8(3)$ \\
\hline N9 & $-2307.9(12)$ & $3120.8(9)$ & $3816.9(8)$ & $12.7(3)$ \\
\hline C68 & $-3487.4(14)$ & $7077.6(11)$ & 4698.8(10) & $14.7(4)$ \\
\hline C73 & $434.7(15)$ & 2844.1(12) & 3899.7(11) & $18.1(4)$ \\
\hline C65 & $68.5(15)$ & $7441.6(12)$ & 4150.1(11) & $18.8(4)$ \\
\hline C20B & $4876.9(18)$ & $9154.9(14)$ & 2920.4(13) & $29.6(5)$ \\
\hline C24B & $-3361.5(15)$ & 9522.1(12) & 107.3(11) & $17.7(4)$ \\
\hline C72 & $-374.8(15)$ & $3219.0(11)$ & $3719.9(10)$ & $14.7(4)$ \\
\hline C3B & $-787.7(14)$ & 11698.1(11) & 2038.2(10) & $13.9(4)$ \\
\hline $\mathrm{C} 23$ & $-3644.3(15)$ & $5074.6(12)$ & $432(1)$ & $18.1(4)$ \\
\hline C7 & $1214.9(14)$ & $3813.0(11)$ & $1192.2(10)$ & $12.0(4)$ \\
\hline C75 & $-2007.5(15)$ & $3985.0(11)$ & $3885.8(10)$ & $14.6(4)$ \\
\hline C64 & $-1094.2(15)$ & $7280.8(12)$ & 3934.8(11) & $18.2(4)$ \\
\hline $\mathrm{C} 24$ & $-3388.7(14)$ & $4441.2(12)$ & 123.6(10) & $16.9(4)$ \\
\hline C84 & $-3543.3(16)$ & $1990.4(12)$ & $4314.2(12)$ & $20.9(4)$ \\
\hline C78 & $-2518.0(16)$ & $5370.8(13)$ & $4633.5(11)$ & $21.7(4)$ \\
\hline C10 & $2830.5(15)$ & $3423.9(12)$ & $546.9(10)$ & $15.4(4)$ \\
\hline C7B & $1255.7(14)$ & 8772.1(11) & $1110.8(10)$ & $12.1(4)$ \\
\hline C21B & $-2661.1(14)$ & 10134.0(11) & $1513.3(10)$ & $13.8(4)$ \\
\hline C61 & $-2281.4(16)$ & $10079.3(12)$ & 4469.9(11) & $19.3(4)$ \\
\hline C3 & $-888.2(14)$ & $6729.0(11)$ & 1936.1(10) & $12.5(4)$ \\
\hline $\mathrm{C} 21$ & $-2700.2(14)$ & $5122.6(11)$ & $1519.6(10)$ & $11.9(4)$ \\
\hline C57 & $-3405.7(17)$ & $7765.2(13)$ & 1918.6(10) & $19.6(4)$ \\
\hline C10B & 2913.8(15) & $8415.1(12)$ & 494.8(10) & $16.3(4)$ \\
\hline C1 & $1497.2(14)$ & 7143.1(11) & 1729.2(9) & $12.3(4)$ \\
\hline $\mathrm{C} 20$ & $5062.5(16)$ & $4562.6(13)$ & 2759.2(12) & $22.6(5)$ \\
\hline C85 & $-4518.8(15)$ & 1779.1(12) & 4716.6(11) & $18.3(4)$ \\
\hline C18 & $4670.2(14)$ & $5791.6(12)$ & 2701.2(11) & $18.5(4)$ \\
\hline $\mathrm{C} 4$ & $-345.7(13)$ & 6586.8(11) & $1280.6(9)$ & $11.2(4)$ \\
\hline C4B & $-247.4(14)$ & 11587.4(11) & $1371.7(10)$ & $12.1(4)$ \\
\hline C56 & $-3348.9(15)$ & $8066.7(11)$ & 2701.5(10) & $14.5(4)$ \\
\hline C5B & 961.5(14) & 10916.7(11) & 937.2(9) & $12.9(4)$ \\
\hline C5 & 915.1(14) & $5941.0(11)$ & $904.1(9)$ & $12.1(4)$ \\
\hline $\mathrm{C} 25$ & $-2789.9(15)$ & $4148.6(12)$ & 535.1(11) & $18.2(4)$ \\
\hline C80 & $-3552.5(15)$ & $2959.4(12)$ & 2725.3(10) & $16.9(4)$ \\
\hline C66 & 814.1(17) & $8020.7(13)$ & $3774.0(11)$ & $23.7(5)$ \\
\hline C8B & 2258.3(15) & $9054.7(11)$ & 1461.4(10) & $15.5(4)$ \\
\hline C13B & $3796.5(17)$ & 8190.1(13) & 177.2(12) & $24.3(5)$ \\
\hline C14 & $3203.8(14)$ & $6036.9(12)$ & $3242(1)$ & $14.1(4)$ \\
\hline C23B & $-3654.8(15)$ & $10120.8(12)$ & 455.7(11) & $19.7(4)$ \\
\hline C70 & $-4591.0(16)$ & $6147.9(13)$ & $5419.7(11)$ & $21.7(4)$ \\
\hline C17 & $4407.0(14)$ & $5073.0(12)$ & 2906.5(10) & $16.7(4)$ \\
\hline
\end{tabular}




\begin{tabular}{|c|c|c|c|c|}
\hline Atom & $\mathbf{x}$ & $\mathbf{y}$ & $\mathbf{z}$ & $U_{e q}$ \\
\hline$\overline{C 9 B}$ & $3073.3(15)$ & $8871.4(12)$ & $1152.0(11)$ & $17.9(4)$ \\
\hline C19 & $4081.2(14)$ & $6268.2(12)$ & $2866.6(10)$ & $17.2(4)$ \\
\hline C8 & 2238.6(15) & 4115.1(11) & $1510.9(10)$ & $15.9(4)$ \\
\hline C67 & $-3344.9(15)$ & 7234.8(11) & $3957.9(10)$ & $14.8(4)$ \\
\hline C77 & $-1442.0(15)$ & $5277.3(11)$ & $4631.0(11)$ & $18.3(4)$ \\
\hline C11B & $1917.8(15)$ & $8156.0(11)$ & $143.3(10)$ & $16.0(4)$ \\
\hline C13 & $3690.2(17)$ & $3189.3(14)$ & $202.3(12)$ & $24.3(5)$ \\
\hline C9 & $3030.9(15)$ & $3914.3(12)$ & $1183.0(11)$ & $18.7(4)$ \\
\hline C69 & $-4447.5(15)$ & $6368.9(12)$ & $4695.5(11)$ & $19.0(4)$ \\
\hline C11 & 1808.1(15) & $3138.2(11)$ & $229.9(10)$ & $15.3(4)$ \\
\hline C6B & $305.4(15)$ & $10080.9(11)$ & 872.4(9) & $13.4(4)$ \\
\hline C27 & $-3745.3(17)$ & $4088.9(14)$ & $-633.4(11)$ & $24.9(5)$ \\
\hline C55 & $-2413.4(15)$ & $7962.9(12)$ & $3086.2(10)$ & $14.8(4)$ \\
\hline C22B & $-3314.5(15)$ & $10424.5(12)$ & $1152.4(11)$ & $17.9(4)$ \\
\hline C1B & $1595.9(14)$ & $12051.2(11)$ & $1839.6(10)$ & $14.5(4)$ \\
\hline C63 & $-1423.0(14)$ & 7971.3(11) & $4188.9(10)$ & $14.2(4)$ \\
\hline C79 & $-2641.0(15)$ & 2777.3(12) & $3051.6(10)$ & $15.4(4)$ \\
\hline C59 & $-2724.6(15)$ & 8652.1(11) & 4234.4(10) & $15.3(4)$ \\
\hline C19B & $3995.5(15)$ & $10899.2(13)$ & $2955.5(11)$ & $19.6(4)$ \\
\hline C14B & $3071.0(15)$ & $10681.8(12)$ & $3284.3(10)$ & $15.6(4)$ \\
\hline C12 & $1006.2(15)$ & $3333.9(11)$ & $549.9(10)$ & $13.5(4)$ \\
\hline $\mathrm{C} 26$ & $-2443.1(14)$ & 4484.1(11) & $1226.2(10)$ & $14.8(4)$ \\
\hline C71 & $-1391.8(14)$ & $2876.9(11)$ & 4049.4(10) & $13.8(4)$ \\
\hline C82 & $-4643.7(17)$ & 2788.6(13) & 1583.7(11) & $23.4(5)$ \\
\hline C22 & $-3312.3(14)$ & $5413.9(12)$ & $1120.2(10)$ & $15.0(4)$ \\
\hline C74 & $1494.3(15)$ & $3247.2(12)$ & $3655.9(11)$ & $18.7(4)$ \\
\hline C81 & $-3782.7(17)$ & $2583.6(13)$ & $1954.7(11)$ & $21.3(4)$ \\
\hline C18B & $4564.9(16)$ & $10399.3(13)$ & 2825.5(11) & $22.3(5)$ \\
\hline C62 & $-1417.5(18)$ & $10840.1(12)$ & $4485.6(12)$ & $25.3(5)$ \\
\hline C16 & $3522.0(15)$ & 4851.2(12) & $3275.7(10)$ & 17.1(4) \\
\hline C27B & $-3720.0(17)$ & 9205.3(14) & $-653.8(11)$ & $24.3(5)$ \\
\hline C15 & $2924.6(15)$ & $5321.0(12)$ & $3440(1)$ & $16.6(4)$ \\
\hline C25B & $-2718.0(15)$ & $9231.4(12)$ & $481.2(11)$ & $18.5(4)$ \\
\hline C15B & $2717.0(15)$ & $9959.3(13)$ & $3465(1)$ & $18.9(4)$ \\
\hline C12B & $1092.0(15)$ & $8334.7(11)$ & $448(1)$ & $13.8(4)$ \\
\hline C83 & $-3184.1(15)$ & 2841.5(11) & 4281.5(10) & $15.1(4)$ \\
\hline C6 & 281.1(15) & $5106.0(11)$ & $885.0(9)$ & $12.3(4)$ \\
\hline $\mathrm{C} 2$ & 2288.8(14) & 6994.0(11) & 2216.8(10) & $13.8(4)$ \\
\hline C17B & 4227.1(15) & 9681.1(13) & $3023.9(11)$ & $20.2(4)$ \\
\hline C16B & $3296.2(16)$ & $9464.6(13)$ & $3338.9(11)$ & $21.2(4)$ \\
\hline C26B & $-2363.2(15)$ & $9534.1(12)$ & $1177.5(11)$ & $16.7(4)$ \\
\hline C58 & $-4319.1(17)$ & $7883.4(13)$ & $1514.6(11)$ & $22.9(5)$ \\
\hline $\mathrm{C} 60$ & $-1877.6(15)$ & $9414.4(11)$ & 4233.1(10) & $15.4(4)$ \\
\hline $\mathrm{C} 2 \mathrm{~B}$ & $2334.7(15)$ & 11819.3(12) & $2303.9(10)$ & $16.7(4)$ \\
\hline C76 & $-1490.5(16)$ & 4441.4(11) & $4592.6(10)$ & $17.1(4)$ \\
\hline C86 & $-4799.7(18)$ & $963.5(13)$ & $4862.5(13)$ & $27.4(5)$ \\
\hline
\end{tabular}

Table 2: Anisotropic Displacement Parameters $\left(\times 10^{4}\right)$ CMW04_057BuZnTsAq. The anisotropic displacement factor exponent takes the form: $-2 \pi^{2}\left[h^{2} a^{* 2} \times U_{11}+\ldots+2 h k a^{*} \times b^{*} \times U_{12}\right]$

\begin{tabular}{lcccccc}
\hline Atom & $\boldsymbol{U}_{\mathbf{1 1}}$ & \multicolumn{1}{c}{$\boldsymbol{U}_{\mathbf{2 2}}$} & $\boldsymbol{U}_{\mathbf{3 3}}$ & \multicolumn{1}{c}{$\boldsymbol{U}_{\mathbf{2 3}}$} & $\boldsymbol{U}_{\mathbf{1 3}}$ & $\boldsymbol{U}_{\mathbf{1 2}}$ \\
\hline Zn1 & $10.14(10)$ & $11.52(11)$ & $7.27(10)$ & $2.35(8)$ & $0.94(7)$ & $4.02(8)$ \\
Zn1B & $12.38(10)$ & $12.69(12)$ & $7.46(11)$ & $1.59(8)$ & $0.82(7)$ & $4.86(8)$ \\
S3 & $9.30(19)$ & $11.4(2)$ & $9.7(2)$ & $1.80(17)$ & $1.42(15)$ & $3.18(16)$ \\
S1 & $16.3(2)$ & $11.0(2)$ & $14.6(2)$ & $4.48(18)$ & $4.25(17)$ & $5.76(18)$ \\
S1B & $15.3(2)$ & $10.0(2)$ & $13.7(2)$ & $2.65(17)$ & $1.95(17)$ & $4.62(17)$ \\
S3B & $12.5(2)$ & $13.0(2)$ & $13.0(2)$ & $2.31(17)$ & $3.45(16)$ & $4.60(17)$
\end{tabular}




\begin{tabular}{|c|c|c|c|c|c|c|}
\hline Atom & $U_{11}$ & $U_{22}$ & $\boldsymbol{U}_{33}$ & $U_{23}$ & $U_{13}$ & $U_{12}$ \\
\hline S2 & $13.8(2)$ & $17.1(2)$ & $10.0(2)$ & $0.53(18)$ & $-3.17(16)$ & $5.29(18)$ \\
\hline S2B & $16.4(2)$ & $21.9(3)$ & $9.7(2)$ & $-2.55(18)$ & $-3.03(17)$ & $9.29(19)$ \\
\hline 01B & $28.3(8)$ & $16.3(7)$ & $17.8(7)$ & $9.4(6)$ & $6.9(6)$ & $10.6(6)$ \\
\hline 01 & $34.0(8)$ & $18.8(8)$ & $17.5(7)$ & $10.7(6)$ & $9.4(6)$ & $14.3(7)$ \\
\hline 05B & $16.3(7)$ & $18.3(8)$ & $22.5(8)$ & $0.4(6)$ & $6.3(6)$ & $7.0(6)$ \\
\hline O2B & $15.2(7)$ & $13.2(7)$ & $28.2(8)$ & $-1.1(6)$ & $-0.1(6)$ & $3.4(6)$ \\
\hline 05 & $12.3(6)$ & $16.4(7)$ & $15.7(7)$ & $-0.8(5)$ & $3.1(5)$ & $5.8(5)$ \\
\hline 03 & $20.5(7)$ & $27.9(8)$ & $9.6(7)$ & $2.7(6)$ & $1.0(5)$ & $11.9(6)$ \\
\hline 02 & $15.5(7)$ & $15.5(7)$ & $32.9(9)$ & $2.2(6)$ & $4.0(6)$ & $4.4(6)$ \\
\hline 01W & $17.6(7)$ & $18.2(7)$ & $13.2(7)$ & $7.5(6)$ & $3.4(5)$ & $8.6(6)$ \\
\hline 01WB & $19.4(7)$ & $23.4(8)$ & $14.2(7)$ & $7.5(6)$ & $5.0(5)$ & $10.6(6)$ \\
\hline 04 & $21.6(7)$ & $21.1(8)$ & $20.8(8)$ & $-1.8(6)$ & $-8.9(6)$ & $3.6(6)$ \\
\hline O3B & $24.0(7)$ & $35.8(9)$ & $9.9(7)$ & $2.9(6)$ & $1.9(5)$ & $17.3(7)$ \\
\hline 06 & $15.1(6)$ & $17.1(7)$ & $15.2(7)$ & $7.0(6)$ & $2.2(5)$ & $3.5(5)$ \\
\hline O6B & $19.6(7)$ & $17.3(7)$ & $18.6(7)$ & $8.0(6)$ & $5.7(5)$ & $4.1(6)$ \\
\hline 04B & $25.4(8)$ & $22.7(8)$ & $21.6(8)$ & $-6.7(6)$ & $-11.1(6)$ & $8.9(7)$ \\
\hline N4 & 21.1(8) & $11.6(8)$ & 7.7(7) & $3.7(6)$ & $2.5(6)$ & 7.1(7) \\
\hline N4B & $20.6(8)$ & $9.9(8)$ & $8.3(7)$ & $2.2(6)$ & $1.3(6)$ & $6.2(6)$ \\
\hline N3B & $12.9(7)$ & $11.9(8)$ & $12.3(8)$ & $3.2(6)$ & $2.9(6)$ & $4.4(6)$ \\
\hline N2B & $13.6(7)$ & $18.4(9)$ & $9.4(8)$ & $0.9(6)$ & $-1.6(6)$ & $4.3(6)$ \\
\hline N2 & $12.7(7)$ & $15.5(8)$ & $9.3(7)$ & $3.5(6)$ & $-0.9(6)$ & $4.0(6)$ \\
\hline N1 & $8.7(7)$ & $8.7(7)$ & $9.1(7)$ & $1.2(6)$ & $0.1(5)$ & $2.4(6)$ \\
\hline N1B & $11.6(7)$ & $10.5(8)$ & $9.1(7)$ & $1.2(6)$ & $0.3(5)$ & $2.9(6)$ \\
\hline N3 & $10.4(7)$ & $11.0(8)$ & $9.6(7)$ & $3.3(6)$ & $1.8(5)$ & $3.8(6)$ \\
\hline N10 & $13.9(7)$ & $13.7(8)$ & $10.6(8)$ & $0.4(6)$ & $1.1(6)$ & $5.0(6)$ \\
\hline N9 & $17.3(8)$ & $11.9(8)$ & $11.4(8)$ & $3.6(6)$ & $1.1(6)$ & $7.2(6)$ \\
\hline C68 & $15.4(9)$ & $12.8(10)$ & $15.6(9)$ & $3.3(7)$ & $0.2(7)$ & $3.8(7)$ \\
\hline C73 & $19.3(10)$ & $18.2(10)$ & $19.9(10)$ & $7.8(8)$ & $3.5(8)$ & $8.0(8)$ \\
\hline C65 & $17.4(9)$ & $19.9(11)$ & $20.9(10)$ & $3.2(8)$ & $0.4(8)$ & $8.7(8)$ \\
\hline C20B & $28.5(12)$ & $34.8(14)$ & $30.9(13)$ & $0.1(11)$ & $-0.5(9)$ & $20.4(11)$ \\
\hline C24B & $11.4(8)$ & $18.2(10)$ & $18.7(10)$ & $2.4(8)$ & $1.7(7)$ & $-1.7(7)$ \\
\hline C72 & $19.4(9)$ & $12.8(10)$ & $13.5(9)$ & $4.5(7)$ & $3.4(7)$ & $6.1(8)$ \\
\hline C3B & $13.9(8)$ & $10.5(9)$ & 17.1(9) & $2.3(7)$ & $2.0(7)$ & $3.8(7)$ \\
\hline C23 & $15.0(9)$ & $21.5(11)$ & $16.3(10)$ & $3.9(8)$ & $-3.3(7)$ & $4.2(8)$ \\
\hline C7 & $15.4(9)$ & $10.8(9)$ & $12.0(9)$ & $5.0(7)$ & $2.9(7)$ & $5.9(7)$ \\
\hline C75 & $20.0(9)$ & $10.3(9)$ & $14.9(9)$ & $3.2(7)$ & $-0.2(7)$ & $6.5(7)$ \\
\hline C64 & 19.1(9) & $13.9(10)$ & $22(1)$ & $1.2(8)$ & $1.6(8)$ & $6.8(8)$ \\
\hline $\mathrm{C} 24$ & $10.4(8)$ & $19.6(11)$ & $14.6(9)$ & $1.0(8)$ & $2.2(7)$ & $-2.9(7)$ \\
\hline C84 & $17.1(9)$ & $16.7(11)$ & $30.4(12)$ & $7.5(9)$ & $7.3(8)$ & $5.5(8)$ \\
\hline C78 & $26.0(11)$ & $19.3(11)$ & $22.2(11)$ & $1.0(9)$ & $1.0(8)$ & $11.9(9)$ \\
\hline C10 & $18.5(9)$ & $15.8(10)$ & $15.0(9)$ & $6.6(8)$ & $2.9(7)$ & $8.0(8)$ \\
\hline C7B & $16.0(9)$ & $8.2(9)$ & $12.1(9)$ & $2.7(7)$ & $0.1(7)$ & $3.7(7)$ \\
\hline C21B & $11.5(8)$ & $12.5(9)$ & $15.8(9)$ & $3.0(7)$ & $1.6(7)$ & $1.4(7)$ \\
\hline C61 & $23.7(10)$ & $18.6(11)$ & $16.9(10)$ & $0.9(8)$ & $1.9(8)$ & $9.9(8)$ \\
\hline C3 & $12.7(8)$ & $11.9(9)$ & $14.5(9)$ & $3.4(7)$ & $1.8(7)$ & $5.6(7)$ \\
\hline C21 & $10.6(8)$ & $11.2(9)$ & $12.5(9)$ & $2.0(7)$ & $1.0(6)$ & $1.4(7)$ \\
\hline C57 & $30.0(11)$ & $20.2(11)$ & $12.0(9)$ & $1.5(8)$ & $0.9(8)$ & $13.5(9)$ \\
\hline C10B & $18.8(9)$ & $14.6(10)$ & $18.1(10)$ & $7.4(8)$ & $5.9(7)$ & $6.3(8)$ \\
\hline $\mathrm{C} 1$ & $12.5(8)$ & $11.8(9)$ & $10.6(9)$ & $4.0(7)$ & $0.5(6)$ & $0.4(7)$ \\
\hline $\mathrm{C} 20$ & $18.5(10)$ & $25.0(12)$ & $25.6(11)$ & $1.8(9)$ & $2.0(8)$ & $10.1(9)$ \\
\hline C85 & $14.6(9)$ & $20.9(11)$ & $17.5(10)$ & $2.8(8)$ & $2.3(7)$ & $3.3(8)$ \\
\hline C18 & $9.6(8)$ & $26.8(12)$ & $18.8(10)$ & $6.9(8)$ & $1.7(7)$ & $4.1(8)$ \\
\hline $\mathrm{C} 4$ & 11.1(8) & $11.5(9)$ & $11.5(9)$ & $4.5(7)$ & $0.0(6)$ & $3.5(7)$ \\
\hline C4B & $14.4(8)$ & $10.2(9)$ & $12.2(9)$ & $3.8(7)$ & $0.2(7)$ & $3.9(7)$ \\
\hline C56 & $19.6(9)$ & $12.3(9)$ & $11.4(9)$ & $2.7(7)$ & $1.9(7)$ & $4.6(8)$ \\
\hline C5B & $15.4(9)$ & $14.7(10)$ & $9.3(9)$ & $1.8(7)$ & $2.7(7)$ & $5.7(7)$ \\
\hline $\mathrm{C} 5$ & $14.3(8)$ & $14.3(9)$ & $8.7(9)$ & $2.2(7)$ & $2.7(6)$ & $5.5(7)$ \\
\hline $\mathrm{C} 25$ & $15.9(9)$ & $14.1(10)$ & $21.3(10)$ & $-3.9(8)$ & $3.0(8)$ & $3.3(8)$ \\
\hline
\end{tabular}




\begin{tabular}{|c|c|c|c|c|c|c|}
\hline Atom & $U_{11}$ & $U_{22}$ & $U_{33}$ & $U_{23}$ & $U_{13}$ & $U_{12}$ \\
\hline C80 & $19.3(9)$ & $15.3(10)$ & $14.2(9)$ & $-1.9(8)$ & $-1.6(7)$ & $5.4(8)$ \\
\hline C66 & $23.4(11)$ & $29.7(13)$ & $19.7(11)$ & $5.5(9)$ & $5.9(8)$ & $10.0(9)$ \\
\hline C8B & $20.2(9)$ & $11.1(9)$ & $13.6(9)$ & $-0.5(7)$ & $-1.4(7)$ & $4.4(8)$ \\
\hline C13B & 21.7(10) & 26.1(12) & $27.8(12)$ & $5.7(9)$ & $7.7(9)$ & $10.5(9)$ \\
\hline C14 & $11.4(8)$ & $19.7(10)$ & $11.6(9)$ & $2.0(7)$ & $-3.2(7)$ & $6.3(7)$ \\
\hline C23B & $15.1(9)$ & $20.0(11)$ & 23.1(11) & $6.8(9)$ & $-2.8(8)$ & $3.5(8)$ \\
\hline C70 & $20.2(10)$ & $21.2(11)$ & $22.4(11)$ & $8.9(9)$ & $3.0(8)$ & $2.3(8)$ \\
\hline C17 & $11.0(8)$ & $24.0(11)$ & $14.2(9)$ & $1.2(8)$ & $-3.0(7)$ & $6.0(8)$ \\
\hline C9B & $15.0(9)$ & $17(1)$ & $19.8(10)$ & $4.0(8)$ & $-0.9(7)$ & $2.3(8)$ \\
\hline C19 & $12.7(9)$ & $18.8(10)$ & $19.5(10)$ & $6.4(8)$ & $-1.7(7)$ & $2.8(8)$ \\
\hline C8 & $19.6(9)$ & 13.1(10) & $12.7(9)$ & $-1.0(7)$ & $-2.6(7)$ & $3.8(8)$ \\
\hline C67 & $16.4(9)$ & $12.6(10)$ & 14.1(9) & $1.4(7)$ & $1.4(7)$ & $3.0(7)$ \\
\hline C77 & $19.3(9)$ & $13.3(10)$ & $20.9(10)$ & $-1.4(8)$ & $3.8(8)$ & $4.8(8)$ \\
\hline C11B & $22.4(10)$ & $12.9(10)$ & $12.0(9)$ & $1.8(7)$ & $1.1(7)$ & $4.9(8)$ \\
\hline C13 & $24.1(11)$ & $27.6(12)$ & $26.0(12)$ & $5.9(9)$ & $7.0(9)$ & 13.8(9) \\
\hline C9 & $15.6(9)$ & $19.6(11)$ & $18.9(10)$ & $1.1(8)$ & $-3.7(7)$ & $4.5(8)$ \\
\hline C69 & 17.1(9) & $19.3(11)$ & 17.4(10) & $4.9(8)$ & $-1.6(7)$ & $0.9(8)$ \\
\hline C11 & $20.9(9)$ & $14.3(10)$ & $11.2(9)$ & $1.5(7)$ & $-0.1(7)$ & $6.8(8)$ \\
\hline C6B & 21.3(9) & $13.0(9)$ & 7.1(8) & $1.1(7)$ & $0.9(7)$ & $7.5(8)$ \\
\hline C27 & $20.8(10)$ & $31.4(13)$ & $16.1(10)$ & $-2.9(9)$ & $-0.1(8)$ & $2.8(9)$ \\
\hline C55 & $18.0(9)$ & $15.6(10)$ & $10.9(9)$ & $1.8(7)$ & $3.9(7)$ & $5.7(8)$ \\
\hline $\mathrm{C} 22 \mathrm{~B}$ & $16.5(9)$ & $15.8(10)$ & $22.2(11)$ & $2.2(8)$ & $1.7(8)$ & $6.8(8)$ \\
\hline C1B & $14.4(9)$ & $11.6(9)$ & $14.1(9)$ & $1.2(7)$ & $1.2(7)$ & $0.0(7)$ \\
\hline C63 & $12.7(8)$ & $14(1)$ & $14.8(9)$ & $0.7(7)$ & $0.1(7)$ & $3.8(7)$ \\
\hline C79 & $21.3(9)$ & $14.8(10)$ & $10.8(9)$ & $1.8(7)$ & $1.4(7)$ & 7.1(8) \\
\hline C59 & $17.5(9)$ & $15.6(10)$ & 13.8(9) & $0.3(7)$ & $2.4(7)$ & $7.6(8)$ \\
\hline C19B & $15.5(9)$ & $23.7(11)$ & $19.2(10)$ & $3.7(8)$ & $1.0(7)$ & $6.0(8)$ \\
\hline C14B & $15.3(9)$ & $21.3(11)$ & $10.3(9)$ & $-2.6(8)$ & $-3.4(7)$ & $9.0(8)$ \\
\hline C12 & $15.5(9)$ & $11.0(9)$ & 13.4(9) & $2.7(7)$ & $-1.2(7)$ & $3.6(7)$ \\
\hline $\mathrm{C} 26$ & $13.4(8)$ & $12.7(9)$ & $18.4(10)$ & $2.0(8)$ & $1.0(7)$ & $4.5(7)$ \\
\hline C71 & $16.2(9)$ & $12.0(9)$ & $16.4(9)$ & $5.2(7)$ & $2.0(7)$ & $7.5(7)$ \\
\hline C82 & $24.3(11)$ & $25.7(12)$ & $17.7(10)$ & $-3.0(9)$ & $-3.0(8)$ & $8.0(9)$ \\
\hline C22 & $15.0(9)$ & $14.7(10)$ & $15.7(10)$ & $1.2(8)$ & $0.6(7)$ & $5.9(7)$ \\
\hline C74 & $19.0(9)$ & $18.8(11)$ & $19.2(10)$ & $2.2(8)$ & $2.7(8)$ & $7.9(8)$ \\
\hline C81 & $28.2(11)$ & $18.6(11)$ & $16.2(10)$ & $-2.3(8)$ & $-3.9(8)$ & $9.1(9)$ \\
\hline C18B & $14.5(9)$ & $31.1(13)$ & $19.9(11)$ & $0.4(9)$ & $2.4(8)$ & $7.0(9)$ \\
\hline C62 & $32.8(12)$ & $14.7(11)$ & $27.2(12)$ & $0.1(9)$ & $-3.0(9)$ & $8.1(9)$ \\
\hline C16 & $14.9(9)$ & $18.9(10)$ & $18(1)$ & $6.5(8)$ & $-0.8(7)$ & $5.0(8)$ \\
\hline $\mathrm{C} 27 \mathrm{~B}$ & $20.7(10)$ & $29.5(13)$ & $18.2(11)$ & $0.4(9)$ & $1.0(8)$ & $3.4(9)$ \\
\hline C15 & $12.6(9)$ & $23.2(11)$ & $15.9(10)$ & $7.5(8)$ & $2.5(7)$ & $6.4(8)$ \\
\hline C25B & $16.6(9)$ & $15.4(10)$ & $21.6(11)$ & $-0.8(8)$ & $3.1(8)$ & $4.2(8)$ \\
\hline C15B & $14.9(9)$ & $27.2(12)$ & $15.7(10)$ & $3.9(8)$ & $0.4(7)$ & $8.2(8)$ \\
\hline C12B & $16.9(9)$ & $9.8(9)$ & $13.9(9)$ & $3.5(7)$ & $-1.1(7)$ & $2.9(7)$ \\
\hline C83 & $15.3(9)$ & $16.4(10)$ & $16.3(10)$ & $5.0(8)$ & $4.5(7)$ & $7.6(8)$ \\
\hline C6 & $18.3(9)$ & $12.1(9)$ & $6.8(8)$ & $1.4(7)$ & $0.3(7)$ & $5.5(7)$ \\
\hline $\mathrm{C} 2$ & $10.7(8)$ & $15.8(10)$ & $12.9(9)$ & $3.7(7)$ & $0.1(7)$ & $0.9(7)$ \\
\hline C17B & 17.1(9) & $25.9(12)$ & $18.1(10)$ & $-1.8(8)$ & $-3.0(8)$ & $10.8(9)$ \\
\hline C16B & $20.9(10)$ & $21.8(11)$ & $22.1(11)$ & $5.3(9)$ & $-1.6(8)$ & $8.5(9)$ \\
\hline $\mathrm{C} 26 \mathrm{~B}$ & $15.7(9)$ & $15.7(10)$ & $19.6(10)$ & $2.1(8)$ & 1.1(7) & $6.8(8)$ \\
\hline C58 & $30.0(11)$ & $26.8(12)$ & $12.5(10)$ & $1.2(8)$ & $-1.1(8)$ & $11.2(9)$ \\
\hline C60 & $19.3(9)$ & $12(1)$ & $16.3(10)$ & $2.3(7)$ & $3.2(7)$ & $6.7(8)$ \\
\hline $\mathrm{C} 2 \mathrm{~B}$ & $13.4(9)$ & $19.6(11)$ & $14.7(9)$ & $1.4(8)$ & $1.1(7)$ & $2.7(8)$ \\
\hline C76 & 20.1(9) & $16.7(10)$ & $15.8(10)$ & $0.2(8)$ & $-2.1(7)$ & $9.3(8)$ \\
\hline C86 & $25.1(11)$ & $23.9(12)$ & $30.3(12)$ & $9.2(10)$ & $7.7(9)$ & $1.4(9)$ \\
\hline
\end{tabular}

Table 3: Bond Lengths in Å for CMW04_057BuZnTsAq. 


\begin{tabular}{|c|c|c|}
\hline Atom & Atom & Length/Å \\
\hline$\overline{Z n 1}$ & 01W & $2.1089(13)$ \\
\hline Zn1 & $\mathrm{N} 4$ & $2.0592(16)$ \\
\hline Zn1 & N2 & $2.0755(16)$ \\
\hline Zn1 & N1 & $2.2327(15)$ \\
\hline Zn1 & N3 & $2.0239(15)$ \\
\hline $\mathrm{Zn} 1 \mathrm{~B}$ & 01WB & $2.1054(14)$ \\
\hline Zn1B & N4B & $2.0536(16)$ \\
\hline Zn1B & N3B & $2.0268(16)$ \\
\hline Zn1B & $\mathrm{N} 2 \mathrm{~B}$ & $2.0560(16)$ \\
\hline Zn1B & $\mathrm{N} 1 \mathrm{~B}$ & $2.2571(16)$ \\
\hline S3 & 05 & $1.4554(14)$ \\
\hline S3 & 06 & $1.4391(14)$ \\
\hline S3 & N3 & $1.5742(15)$ \\
\hline S3 & $\mathrm{C} 21$ & $1.7825(19)$ \\
\hline S1 & 01 & $1.4585(15)$ \\
\hline S1 & 02 & $1.4468(15)$ \\
\hline S1 & N4 & $1.5695(17)$ \\
\hline S1 & $\mathrm{C} 7$ & $1.7786(19)$ \\
\hline S1B & 01B & $1.4530(15)$ \\
\hline S1B & O2B & $1.4505(14)$ \\
\hline S1B & $\mathrm{N} 4 \mathrm{~B}$ & $1.5667(17)$ \\
\hline S1B & C7B & $1.7820(19)$ \\
\hline S3B & 05B & $1.4518(14)$ \\
\hline S3B & 06B & $1.4421(15)$ \\
\hline S3B & N3B & $1.5746(16)$ \\
\hline S3B & $\mathrm{C} 21 \mathrm{~B}$ & $1.785(2)$ \\
\hline S2 & 03 & $1.4575(14)$ \\
\hline S2 & 04 & $1.4450(15)$ \\
\hline S2 & N2 & $1.5657(16)$ \\
\hline S2 & C14 & $1.777(2)$ \\
\hline S2B & O3B & $1.4550(15)$ \\
\hline S2B & 04B & $1.4431(16)$ \\
\hline S2B & N2B & $1.5673(16)$ \\
\hline S2B & C14B & $1.784(2)$ \\
\hline N4 & C6 & $1.477(2)$ \\
\hline N4B & C6B & $1.480(2)$ \\
\hline N3B & C3B & $1.474(2)$ \\
\hline $\mathrm{N} 2 \mathrm{~B}$ & $\mathrm{C} 2 \mathrm{~B}$ & $1.476(2)$ \\
\hline N2 & $\mathrm{C} 2$ & $1.474(2)$ \\
\hline N1 & $\mathrm{C} 1$ & $1.474(2)$ \\
\hline N1 & $\mathrm{C} 4$ & $1.474(2)$ \\
\hline N1 & $\mathrm{C} 5$ & $1.474(2)$ \\
\hline N1B & C4B & $1.473(2)$ \\
\hline N1B & C5B & $1.471(2)$ \\
\hline N1B & C1B & $1.475(2)$ \\
\hline N3 & C3 & $1.480(2)$ \\
\hline N10 & C67 & $1.525(2)$ \\
\hline N10 & C55 & $1.523(2)$ \\
\hline N10 & C63 & $1.516(2)$ \\
\hline N10 & C59 & $1.525(2)$ \\
\hline N9 & $\mathrm{C} 75$ & $1.521(2)$ \\
\hline N9 & C79 & $1.518(2)$ \\
\hline N9 & C71 & $1.520(2)$ \\
\hline N9 & C83 & $1.518(2)$ \\
\hline C68 & C67 & $1.513(3)$ \\
\hline C68 & C69 & $1.532(3)$ \\
\hline C73 & C72 & $1.526(3)$ \\
\hline C73 & C74 & $1.519(3)$ \\
\hline
\end{tabular}

\begin{tabular}{|c|c|c|}
\hline Atom & Atom & Length/Å \\
\hline C65 & C64 & $1.530(3)$ \\
\hline C65 & C66 & $1.520(3)$ \\
\hline C20B & C17B & $1.509(3)$ \\
\hline C24B & C23B & $1.396(3)$ \\
\hline C24B & $\mathrm{C} 27 \mathrm{~B}$ & $1.507(3)$ \\
\hline C24B & C25B & $1.391(3)$ \\
\hline C72 & C71 & $1.526(3)$ \\
\hline C3B & $\mathrm{C} 4 \mathrm{~B}$ & $1.527(3)$ \\
\hline C23 & $\mathrm{C} 24$ & $1.396(3)$ \\
\hline C23 & $\mathrm{C} 22$ & $1.383(3)$ \\
\hline C7 & $\mathrm{C} 8$ & $1.396(3)$ \\
\hline C7 & C12 & $1.387(3)$ \\
\hline C75 & C76 & $1.518(3)$ \\
\hline C64 & C63 & $1.517(3)$ \\
\hline C24 & $\mathrm{C} 25$ & $1.393(3)$ \\
\hline C24 & $\mathrm{C} 27$ & $1.511(3)$ \\
\hline C84 & C85 & $1.528(3)$ \\
\hline C84 & C83 & $1.521(3)$ \\
\hline C78 & C77 & $1.516(3)$ \\
\hline C10 & C13 & $1.510(3)$ \\
\hline C10 & $\mathrm{C} 9$ & $1.388(3)$ \\
\hline C10 & C11 & $1.393(3)$ \\
\hline C7B & C8B & $1.395(3)$ \\
\hline C7B & C12B & $1.389(3)$ \\
\hline C21B & $\mathrm{C} 22 \mathrm{~B}$ & $1.387(3)$ \\
\hline C21B & C26B & $1.392(3)$ \\
\hline C61 & C62 & $1.523(3)$ \\
\hline C61 & $\mathrm{C} 60$ & $1.528(3)$ \\
\hline C3 & $\mathrm{C} 4$ & $1.522(2)$ \\
\hline C21 & $\mathrm{C} 26$ & $1.396(3)$ \\
\hline C21 & $\mathrm{C} 22$ & $1.390(3)$ \\
\hline C57 & C56 & $1.530(3)$ \\
\hline C57 & C58 & $1.526(3)$ \\
\hline C10B & C13B & $1.507(3)$ \\
\hline C10B & C9B & $1.395(3)$ \\
\hline C10B & C11B & $1.389(3)$ \\
\hline C1 & $\mathrm{C} 2$ & $1.515(3)$ \\
\hline C20 & C17 & $1.499(3)$ \\
\hline C85 & C86 & $1.522(3)$ \\
\hline C18 & C17 & $1.397(3)$ \\
\hline C18 & C19 & $1.383(3)$ \\
\hline C56 & C55 & $1.520(3)$ \\
\hline C5B & C6B & $1.518(3)$ \\
\hline $\mathrm{C} 5$ & $\mathrm{C} 6$ & $1.519(3)$ \\
\hline $\mathrm{C} 25$ & $\mathrm{C} 26$ & $1.389(3)$ \\
\hline C80 & C79 & $1.515(3)$ \\
\hline C80 & C81 & $1.531(3)$ \\
\hline C8B & C9B & $1.384(3)$ \\
\hline C14 & C19 & $1.396(3)$ \\
\hline C14 & C15 & $1.386(3)$ \\
\hline C23B & $\mathrm{C} 22 \mathrm{~B}$ & $1.386(3)$ \\
\hline C70 & C69 & $1.521(3)$ \\
\hline C17 & C16 & $1.395(3)$ \\
\hline C8 & C9 & $1.387(3)$ \\
\hline C77 & C76 & $1.534(3)$ \\
\hline C11B & C12B & $1.389(3)$ \\
\hline C11 & C12 & $1.387(3)$ \\
\hline C1B & $\mathrm{C} 2 \mathrm{~B}$ & $1.519(3)$ \\
\hline
\end{tabular}




\begin{tabular}{lll}
\hline Atom & Atom & Length/A \\
\hline C59 & C60 & $1.520(3)$ \\
C19B & C14B & $1.393(3)$ \\
C19B & C18B & $1.388(3)$ \\
C14B & C15B & $1.384(3)$ \\
C82 & C81 & $1.520(3)$
\end{tabular}

\begin{tabular}{lll}
\hline Atom & Atom & Length/Å \\
\hline C18B & C17B & $1.390(3)$ \\
C16 & C15 & $1.381(3)$ \\
C25B & C26B & $1.389(3)$ \\
C15B & C16B & $1.390(3)$ \\
C17B & C16B & $1.386(3)$
\end{tabular}

Table 4: Bond Angles in ${ }^{\circ}$ for CMW04_057BuZnTsAq.

\begin{tabular}{|c|c|c|c|c|c|c|c|}
\hline Atom & Atom & Atom & Angle $/^{\circ}$ & Atom & Atom & Atom & Angle $/^{\circ}$ \\
\hline $01 \mathrm{~W}$ & Zn1 & N1 & $174.29(5)$ & 04 & S2 & N2 & $113.00(9)$ \\
\hline $\mathrm{N} 4$ & $\mathrm{Zn} 1$ & 01W & $97.49(6)$ & 04 & S2 & C14 & $105.35(9)$ \\
\hline N4 & $\mathrm{Zn} 1$ & $\mathrm{~N} 2$ & $115.64(6)$ & $\mathrm{N} 2$ & S2 & C14 & $108.24(9)$ \\
\hline N4 & Zn1 & N1 & $80.86(6)$ & O3B & S2B & $\mathrm{N} 2 \mathrm{~B}$ & 108.33(9) \\
\hline N2 & Zn1 & $01 \mathrm{~W}$ & $95.57(6)$ & O3B & S2B & C14B & $105.11(9)$ \\
\hline N2 & Zn1 & N1 & $80.34(6)$ & $04 \mathrm{~B}$ & S2B & 03B & $116.57(9)$ \\
\hline N3 & Zn1 & $01 \mathrm{~W}$ & $103.80(6)$ & 04B & S2B & N2B & $112.16(9)$ \\
\hline N3 & Zn1 & N4 & $117.75(6)$ & $04 \mathrm{~B}$ & S2B & C14B & $104.27(9)$ \\
\hline N3 & Zn1 & N2 & $119.39(6)$ & $\mathrm{N} 2 \mathrm{~B}$ & S2B & C14B & $109.99(9)$ \\
\hline N3 & Zn1 & N1 & $81.75(6)$ & S1 & N4 & Zn1 & $130.89(9)$ \\
\hline O1WB & Zn1B & N1B & $174.61(5)$ & C6 & N4 & Zn1 & $112.35(12)$ \\
\hline N4B & Zn1B & 01WB & $97.33(6)$ & C6 & N4 & S1 & $114.24(12)$ \\
\hline N4B & Zn1B & N2B & $113.72(7)$ & S1B & N4B & Zn1B & $130.94(9)$ \\
\hline N4B & Zn1B & N1B & $80.33(6)$ & C6B & N4B & Zn1B & $112.79(12)$ \\
\hline N3B & Zn1B & 01WB & $103.85(6)$ & C6B & $\mathrm{N} 4 \mathrm{~B}$ & S1B & $114.08(12)$ \\
\hline N3B & Zn1B & N4B & $119.49(6)$ & S3B & N3B & Zn1B & $127.00(10)$ \\
\hline N3B & Zn1B & $\mathrm{N} 2 \mathrm{~B}$ & $119.22(7)$ & C3B & N3B & Zn1B & $111.76(11)$ \\
\hline N3B & Zn1B & $\mathrm{N} 1 \mathrm{~B}$ & $81.50(6)$ & C3B & N3B & S3B & $115.16(12)$ \\
\hline N2B & $\mathrm{Zn} 1 \mathrm{~B}$ & 01WB & $96.17(6)$ & S2B & N2B & $\mathrm{Zn} 1 \mathrm{~B}$ & $128.54(10)$ \\
\hline N2B & Zn1B & N1B & $80.47(6)$ & $\mathrm{C} 2 \mathrm{~B}$ & N2B & Zn1B & $113.02(12)$ \\
\hline 05 & S3 & N3 & $111.49(8)$ & $\mathrm{C} 2 \mathrm{~B}$ & $\mathrm{~N} 2 \mathrm{~B}$ & S2B & $115.21(13)$ \\
\hline 05 & S3 & $\mathrm{C} 21$ & $104.53(8)$ & S2 & N2 & Zn1 & 129.44(9) \\
\hline 06 & S3 & 05 & $117.03(8)$ & $\mathrm{C} 2$ & N2 & Zn1 & $112.64(11)$ \\
\hline 06 & S3 & N3 & 109.16(8) & $\mathrm{C} 2$ & N2 & $\mathrm{S} 2$ & $114.45(12)$ \\
\hline 06 & S3 & $\mathrm{C} 21$ & $106.81(9)$ & C1 & N1 & Zn1 & $106.76(10)$ \\
\hline N3 & S3 & $\mathrm{C} 21$ & $107.19(8)$ & C1 & N1 & C4 & $111.84(15)$ \\
\hline 01 & S1 & N4 & $107.73(9)$ & C4 & N1 & Zn1 & $106.32(10)$ \\
\hline 01 & S1 & C7 & $106.26(9)$ & C5 & N1 & Zn1 & $106.58(11)$ \\
\hline 02 & S1 & 01 & $115.53(9)$ & $\mathrm{C} 5$ & N1 & C1 & $112.15(14)$ \\
\hline 02 & S1 & N4 & $114.59(9)$ & C5 & N1 & $\mathrm{C} 4$ & $112.68(14)$ \\
\hline 02 & S1 & C7 & $104.79(9)$ & C4B & N1B & Zn1B & $105.95(11)$ \\
\hline N4 & S1 & C7 & $107.29(9)$ & C4B & N1B & C1B & $112.32(15)$ \\
\hline 01B & S1B & N4B & 108.41(8) & C5B & N1B & Zn1B & $106.89(11)$ \\
\hline 01B & S1B & C7B & $106.09(9)$ & C5B & N1B & C4B & $113.05(14)$ \\
\hline O2B & S1B & 01B & $115.77(9)$ & C5B & N1B & C1B & $111.94(14)$ \\
\hline O2B & S1B & N4B & $114.08(9)$ & C1B & N1B & Zn1B & $106.10(11)$ \\
\hline O2B & S1B & C7B & 104.71(9) & S3 & N3 & Zn1 & 127.99(9) \\
\hline N4B & S1B & C7B & $107.08(9)$ & C3 & N3 & Zn1 & $111.57(11)$ \\
\hline 05B & S3B & N3B & $112.04(9)$ & C3 & N3 & S3 & $114.90(12)$ \\
\hline 05B & S3B & C21B & 104.91(9) & C55 & N10 & C67 & $107.23(14)$ \\
\hline O6B & S3B & 05B & $117.08(9)$ & C55 & N10 & C59 & $111.84(15)$ \\
\hline O6B & S3B & N3B & $108.66(9)$ & C63 & N10 & C67 & $111.27(15)$ \\
\hline O6B & S3B & C21B & $105.99(9)$ & C63 & N10 & C55 & $108.82(14)$ \\
\hline N3B & S3B & C21B & $107.52(9)$ & C63 & N10 & C59 & $108.68(14)$ \\
\hline 03 & $\mathrm{~S} 2$ & $\mathrm{~N} 2$ & $108.12(8)$ & C59 & N10 & C67 & $109.03(14)$ \\
\hline 03 & S2 & C14 & $105.28(9)$ & C79 & N9 & C75 & $108.31(14)$ \\
\hline 04 & S2 & 03 & $116.29(9)$ & C79 & N9 & C71 & $108.23(14)$ \\
\hline
\end{tabular}




\begin{tabular}{|c|c|c|c|c|c|c|c|}
\hline Atom & Atom & Atom & Angle $/^{\circ}$ & Atom & Atom & Atom & Angle $/^{\circ}$ \\
\hline C79 & N9 & C83 & $\overline{111.90(15)}$ & C19 & C14 & S2 & $120.06(16)$ \\
\hline C71 & N9 & C75 & $111.75(14)$ & C15 & C14 & $\mathrm{S} 2$ & $120.74(15)$ \\
\hline C83 & N9 & C75 & $108.67(14)$ & C15 & C14 & C19 & $119.17(18)$ \\
\hline C83 & N9 & C71 & $108.02(14)$ & C22B & C23B & C24B & $121.46(19)$ \\
\hline C67 & C68 & C69 & $109.44(16)$ & C18 & C17 & C20 & $121.83(18)$ \\
\hline C74 & C73 & C72 & $111.25(16)$ & C16 & C17 & C20 & $120.58(19)$ \\
\hline C66 & C65 & C64 & $114.45(18)$ & C16 & C17 & C18 & $117.56(19)$ \\
\hline C23B & C24B & C27B & $121.05(19)$ & C8B & C9B & C10B & $121.33(18)$ \\
\hline C25B & C24B & C23B & 118.04(19) & C18 & C19 & C14 & $120.36(19)$ \\
\hline C25B & C24B & C27B & $120.9(2)$ & C9 & C8 & C7 & $118.98(18)$ \\
\hline C71 & C72 & C73 & $110.70(16)$ & C68 & C67 & N10 & $116.51(15)$ \\
\hline N3B & C3B & C4B & $109.78(15)$ & C78 & C77 & C76 & $112.55(17)$ \\
\hline $\mathrm{C} 22$ & $\mathrm{C} 23$ & $\mathrm{C} 24$ & 121.94(19) & C10B & C11B & C12B & $120.53(18)$ \\
\hline C8 & C7 & S1 & $119.59(15)$ & C8 & C9 & C10 & $121.60(18)$ \\
\hline C12 & C7 & S1 & $120.35(14)$ & C70 & C69 & C68 & $112.05(16)$ \\
\hline C12 & C7 & C8 & $120.06(17)$ & C12 & C11 & C10 & $120.48(18)$ \\
\hline C76 & C75 & N9 & $116.24(16)$ & N4B & C6B & C5B & $108.72(15)$ \\
\hline C63 & C64 & C65 & $110.69(16)$ & C56 & C55 & N10 & $115.62(15)$ \\
\hline $\mathrm{C} 23$ & C24 & C27 & $120.94(19)$ & C23B & $\mathrm{C} 22 \mathrm{~B}$ & $\mathrm{C} 21 \mathrm{~B}$ & $119.63(19)$ \\
\hline C25 & C24 & C23 & $117.83(18)$ & N1B & C1B & C2B & $110.52(16)$ \\
\hline C25 & C24 & C27 & $121.2(2)$ & N10 & C63 & C64 & $115.83(16)$ \\
\hline C83 & C84 & C85 & $109.96(17)$ & C80 & C79 & N9 & $115.87(16)$ \\
\hline C9 & C10 & C13 & $121.39(18)$ & C60 & C59 & N10 & $114.62(15)$ \\
\hline C9 & C10 & C11 & $118.69(18)$ & C18B & C19B & C14B & $120.0(2)$ \\
\hline C11 & C10 & C13 & $119.92(18)$ & C19B & C14B & S2B & $119.41(17)$ \\
\hline C8B & C7B & S1B & $119.79(15)$ & C15B & C14B & S2B & $121.18(15)$ \\
\hline C12B & C7B & S1B & $120.24(14)$ & C15B & C14B & C19B & $119.39(19)$ \\
\hline C12B & C7B & C8B & $119.97(18)$ & C11 & C12 & C7 & $120.17(17)$ \\
\hline $\mathrm{C} 22 \mathrm{~B}$ & $\mathrm{C} 21 \mathrm{~B}$ & S3B & $121.45(16)$ & $\mathrm{C} 25$ & $\mathrm{C} 26$ & $\mathrm{C} 21$ & $119.89(18)$ \\
\hline C22B & $\mathrm{C} 21 \mathrm{~B}$ & C26B & $119.90(18)$ & N9 & C71 & C72 & $115.21(15)$ \\
\hline C26B & C21B & S3B & $118.61(15)$ & C23 & $\mathrm{C} 22$ & $\mathrm{C} 21$ & $119.47(19)$ \\
\hline C62 & C61 & C60 & $110.51(17)$ & C82 & C81 & $\mathrm{C} 80$ & $112.20(18)$ \\
\hline N3 & C3 & C4 & $109.35(15)$ & C19B & C18B & C17B & $120.74(19)$ \\
\hline C26 & C21 & S3 & $119.38(14)$ & C15 & C16 & C17 & 121.83(19) \\
\hline $\mathrm{C} 22$ & $\mathrm{C} 21$ & S3 & $120.77(15)$ & C16 & C15 & C14 & $119.99(18)$ \\
\hline C22 & $\mathrm{C} 21$ & $\mathrm{C} 26$ & 119.74(18) & C26B & C25B & C24B & $121.16(19)$ \\
\hline C58 & C57 & C56 & $111.45(17)$ & C14B & C15B & C16B & $120.22(19)$ \\
\hline С9B & C10B & C13B & $120.85(19)$ & C7B & C12B & C11B & $120.11(18)$ \\
\hline C11B & C10B & C13B & $120.38(19)$ & N9 & C83 & C84 & $116.11(16)$ \\
\hline C11B & C10B & C9B & $118.75(18)$ & N4 & $\mathrm{C} 6$ & $\mathrm{C} 5$ & $108.38(15)$ \\
\hline N1 & C1 & $\mathrm{C} 2$ & $110.68(15)$ & N2 & $\mathrm{C} 2$ & $\mathrm{C} 1$ & $108.51(14)$ \\
\hline C86 & C85 & $\mathrm{C} 84$ & $111.52(18)$ & C18B & C17B & C20B & $120.78(19)$ \\
\hline C19 & C18 & $\mathrm{C} 17$ & $121.08(18)$ & C16B & C17B & C20B & $120.4(2)$ \\
\hline N1 & $\mathrm{C} 4$ & C3 & $109.79(14)$ & C16B & C17B & C18B & $118.8(2)$ \\
\hline N1B & C4B & C3B & $109.75(15)$ & $\mathrm{C} 17 \mathrm{~B}$ & C16B & C15B & $120.8(2)$ \\
\hline C55 & C56 & $\mathrm{C} 57$ & $110.23(16)$ & C25B & $\mathrm{C} 26 \mathrm{~B}$ & $\mathrm{C} 21 \mathrm{~B}$ & 119.81(19) \\
\hline N1B & C5B & C6B & $111.06(15)$ & C59 & C60 & C61 & $110.97(16)$ \\
\hline N1 & C5 & C6 & $110.97(14)$ & $\mathrm{N} 2 \mathrm{~B}$ & $\mathrm{C} 2 \mathrm{~B}$ & C1B & $108.81(15)$ \\
\hline C26 & $\mathrm{C} 25$ & $\mathrm{C} 24$ & 121.11(19) & C75 & C76 & C77 & $110.03(16)$ \\
\hline C79 & $\mathrm{C} 80$ & C81 & $109.61(16)$ & & & & \\
\hline C9B & C8B & C7B & $119.26(18)$ & & & & \\
\hline
\end{tabular}

Table 5: Hydrogen Fractional Atomic Coordinates $\left(\times 10^{4}\right)$ and Equivalent Isotropic Displacement Parameters $\left(\AA^{2} \times 10^{3}\right)$ for CMW04_057BuZnTsAq. $U_{\text {eq }}$ is defined as $1 / 3$ of the trace of the orthogonalised $U_{i j}$. 


\begin{tabular}{|c|c|c|c|c|}
\hline Atom & & & & $U_{e q}$ \\
\hline$\overline{\mathrm{H} 1 \mathrm{WA}}$ & 645 & 5414 & 3549 & 23 \\
\hline H1WB & 189 & 4710 & 3070 & 23 \\
\hline H1WC & 468 & 10183 & 3538 & 26 \\
\hline H1WD & 170 & 9516 & 3006 & 26 \\
\hline H68A & -3585 & 7523 & 4995 & 18 \\
\hline H68B & -2854 & 6992 & 4897 & 18 \\
\hline $\mathrm{H} 73 \mathrm{~A}$ & 508 & 2860 & 4413 & 22 \\
\hline H73B & 189 & 2301 & 3673 & 22 \\
\hline H65A & 202 & 7629 & 4661 & 23 \\
\hline H65B & 226 & 6956 & 4059 & 23 \\
\hline H20D & 4743 & 8885 & 2436 & 44 \\
\hline $\mathrm{H} 20 \mathrm{E}$ & 4688 & 8783 & 3242 & 44 \\
\hline $\mathrm{H} 20 \mathrm{~F}$ & 5622 & 9455 & 3015 & 44 \\
\hline $\mathrm{H} 72 \mathrm{~A}$ & -91 & 3775 & 3894 & 18 \\
\hline H72B & -520 & 3143 & 3205 & 18 \\
\hline H3BA & -344 & 12160 & 2358 & 17 \\
\hline Н3ВВ & -1466 & 11771 & 1920 & 17 \\
\hline H23 & -4059 & 5279 & 161 & 22 \\
\hline $\mathrm{H} 75 \mathrm{~A}$ & -1525 & 4154 & 3525 & 18 \\
\hline H75B & -2649 & 4114 & 3780 & 18 \\
\hline H64A & -1223 & 7152 & 3418 & 22 \\
\hline H64B & -1521 & 6837 & 4136 & 22 \\
\hline H84A & -2975 & 1860 & 4550 & 25 \\
\hline H84B & -3704 & 1695 & 3834 & 25 \\
\hline H78A & -2892 & 5173 & 4167 & 33 \\
\hline H78B & -2442 & 5913 & 4759 & 33 \\
\hline H78C & -2916 & 5086 & 4977 & 33 \\
\hline H61A & -2883 & 10048 & 4144 & 23 \\
\hline H61B & -2528 & 10045 & 4943 & 23 \\
\hline H3A & -467 & 7219 & 2228 & 15 \\
\hline H3B & -1586 & 6763 & 1804 & 15 \\
\hline H57A & -2741 & 8033 & 1731 & 24 \\
\hline H57B & -3490 & 7213 & 1851 & 24 \\
\hline $\mathrm{H} 1 \mathrm{~A}$ & 1218 & 7527 & 1981 & 15 \\
\hline $\mathrm{H} 1 \mathrm{~B}$ & 1846 & 7352 & 1327 & 15 \\
\hline $\mathrm{H} 20 \mathrm{~A}$ & 5308 & 4595 & 2291 & 34 \\
\hline H20B & 4641 & 4034 & 2781 & 34 \\
\hline $\mathrm{H} 20 \mathrm{C}$ & 5667 & 4725 & 3109 & 34 \\
\hline H85A & -4392 & 2135 & 5166 & 22 \\
\hline H85B & -5116 & 1834 & 4441 & 22 \\
\hline H18 & 5263 & 5955 & 2444 & 22 \\
\hline $\mathrm{H} 4 \mathrm{~A}$ & -819 & 6145 & 953 & 13 \\
\hline $\mathrm{H} 4 \mathrm{~B}$ & -178 & 7040 & 1043 & 13 \\
\hline H4BA & -737 & 11178 & 1021 & 15 \\
\hline H4BB & -44 & 12066 & 1170 & 15 \\
\hline H56A & -3283 & 8615 & 2771 & 17 \\
\hline H56B & -4002 & 7788 & 2895 & 17 \\
\hline H5BA & 1714 & 10969 & 1000 & 15 \\
\hline H5BB & 842 & 11107 & 501 & 15 \\
\hline $\mathrm{H} 5 \mathrm{~A}$ & 1672 & 6009 & 977 & 15 \\
\hline H5B & 787 & 6095 & 449 & 15 \\
\hline $\mathrm{H} 25$ & -2616 & 3712 & 340 & 22 \\
\hline H80A & -3379 & 3520 & 2761 & 20 \\
\hline
\end{tabular}




\begin{tabular}{|c|c|c|c|c|}
\hline Atom & & & & $U_{e q}$ \\
\hline $\begin{array}{l}\text { H80B } \\
\text {. }\end{array}$ & -4181 & 2767 & 2980 & 20 \\
\hline H66A & 1539 & 8066 & 3917 & 36 \\
\hline H66B & 713 & 8518 & 3896 & 36 \\
\hline H66C & 672 & 7851 & 3267 & 36 \\
\hline H8B & 2380 & 9369 & 1908 & 19 \\
\hline H13D & 4468 & 8578 & 361 & 36 \\
\hline $\mathrm{H} 13 \mathrm{E}$ & 3712 & 8150 & -333 & 36 \\
\hline $\mathrm{H} 13 \mathrm{~F}$ & 3784 & 7696 & 297 & 36 \\
\hline $\mathrm{H} 23 \mathrm{~B}$ & -4097 & 10325 & 210 & 24 \\
\hline $\mathrm{H} 70 \mathrm{~A}$ & -4620 & 6587 & 5752 & 33 \\
\hline H70B & -4000 & 5991 & 5570 & 33 \\
\hline H70C & -5246 & 5723 & 5404 & 33 \\
\hline Н9B & 3755 & 9060 & 1392 & 21 \\
\hline H19 & 4275 & 6756 & 2724 & 21 \\
\hline H8 & 2390 & 4453 & 1946 & 19 \\
\hline H67A & -4014 & 7262 & 3755 & 18 \\
\hline H67B & -3208 & 6794 & 3679 & 18 \\
\hline H77A & -1008 & 5583 & 5062 & 22 \\
\hline H77B & -1097 & 5479 & 4225 & 22 \\
\hline H11B & 1800 & 7854 & -309 & 19 \\
\hline $\mathrm{H} 13 \mathrm{~A}$ & 4374 & 3551 & 401 & 37 \\
\hline H13B & 3612 & 3190 & -303 & 37 \\
\hline $\mathrm{H} 13 \mathrm{C}$ & 3643 & 2675 & 285 & 37 \\
\hline H9 & 3728 & 4117 & 1399 & 22 \\
\hline H69A & -5082 & 6471 & 4523 & 23 \\
\hline H69B & -4368 & 5936 & 4369 & 23 \\
\hline H11 & 1659 & 2807 & -209 & 18 \\
\hline H6BA & -432 & 10011 & 719 & 16 \\
\hline H6BB & 573 & 9767 & 519 & 16 \\
\hline $\mathrm{H} 27 \mathrm{~A}$ & -4481 & 4049 & -731 & 37 \\
\hline H27B & -3676 & 3578 & -722 & 37 \\
\hline $\mathrm{H} 27 \mathrm{C}$ & -3311 & 4411 & -939 & 37 \\
\hline H55A & -1775 & 8379 & 3017 & 18 \\
\hline H55B & -2332 & 7474 & 2868 & 18 \\
\hline $\mathrm{H} 22 \mathrm{~B}$ & -3528 & 10829 & 1381 & 21 \\
\hline H1BA & 1344 & 12435 & 2119 & 17 \\
\hline H1BB & 1976 & 12287 & 1459 & 17 \\
\hline $\mathrm{H} 63 \mathrm{~A}$ & -1445 & 8020 & 4703 & 17 \\
\hline H63B & -877 & 8433 & 4091 & 17 \\
\hline H79A & -2030 & 2957 & 2779 & 18 \\
\hline H79B & -2829 & 2215 & 3006 & 18 \\
\hline H59A & -3385 & 8665 & 4003 & 18 \\
\hline H59B & -2847 & 8588 & 4726 & 18 \\
\hline H19B & 4236 & 11390 & 2820 & 23 \\
\hline H12 & 312 & 3139 & 328 & 16 \\
\hline $\mathrm{H} 26$ & -2032 & 4279 & 1499 & 18 \\
\hline H71A & -1599 & 2313 & 3937 & 17 \\
\hline H71B & -1254 & 3020 & 4565 & 17 \\
\hline H82A & -4773 & 2530 & 1096 & 35 \\
\hline H82B & -4426 & 3343 & 1596 & 35 \\
\hline H82C & -5288 & 2625 & 1820 & 35 \\
\hline $\mathrm{H} 22$ & -3501 & 5843 & 1318 & 18 \\
\hline H74A & 1409 & 3280 & 3158 & 28 \\
\hline
\end{tabular}




\begin{tabular}{|c|c|c|c|c|}
\hline Atom & & & & $\boldsymbol{U}_{e q}$ \\
\hline $\begin{array}{l}\text { H7B } \\
\text {. }\end{array}$ & 1973 & 2959 & 3725 & 28 \\
\hline $\mathrm{H} 74 \mathrm{C}$ & 1784 & 3763 & 3927 & 28 \\
\hline H81A & -3135 & 2748 & 1713 & 26 \\
\hline H81B & -3996 & 2022 & 1926 & 26 \\
\hline H18B & 5192 & 10550 & 2599 & 27 \\
\hline H62A & -819 & 10867 & 4803 & 38 \\
\hline H62B & -1683 & 11260 & 4651 & 38 \\
\hline H62C & -1195 & 10883 & 4013 & 38 \\
\hline H16 & 3325 & 4363 & 3418 & 20 \\
\hline H27D & -4438 & 9205 & -751 & 36 \\
\hline H27E & -3700 & 8681 & -758 & 36 \\
\hline $\mathrm{H} 27 \mathrm{~F}$ & -3254 & 9524 & -947 & 36 \\
\hline H15 & 2323 & 5153 & 3689 & 20 \\
\hline H25B & -2518 & 8819 & 256 & 22 \\
\hline H15B & 2076 & 9801 & 3676 & 23 \\
\hline H12B & 414 & 8157 & 203 & 17 \\
\hline H83A & -3796 & 2974 & 4115 & 18 \\
\hline H83B & -2950 & 3125 & 4763 & 18 \\
\hline H6A & -463 & 5015 & 732 & 15 \\
\hline H6B & 553 & 4777 & 550 & 15 \\
\hline $\mathrm{H} 2 \mathrm{~A}$ & 2658 & 6679 & 1950 & 17 \\
\hline H2B & 2816 & 7484 & 2438 & 17 \\
\hline H16B & 3051 & 8972 & 3470 & 25 \\
\hline H26B & -1919 & 9332 & 1424 & 20 \\
\hline H58A & -4976 & 7632 & 1709 & 34 \\
\hline H58B & -4355 & 7663 & 1020 & 34 \\
\hline H58C & -4214 & 8432 & 1555 & 34 \\
\hline $\mathrm{H} 60 \mathrm{~A}$ & -1649 & 9442 & 3755 & 19 \\
\hline H60B & -1263 & 9455 & 4551 & 19 \\
\hline H2BA & 2680 & 11503 & 2014 & 20 \\
\hline H2BB & 2885 & 12280 & 2557 & 20 \\
\hline H76A & -772 & 4416 & 4661 & 21 \\
\hline H76B & -1896 & 4220 & 4970 & 21 \\
\hline H86A & -4916 & 610 & 4419 & 41 \\
\hline H86B & -5440 & 841 & 5107 & 41 \\
\hline H86C & -4224 & 914 & 5156 & 41 \\
\hline
\end{tabular}




\section{Crystal Data and Experimental}

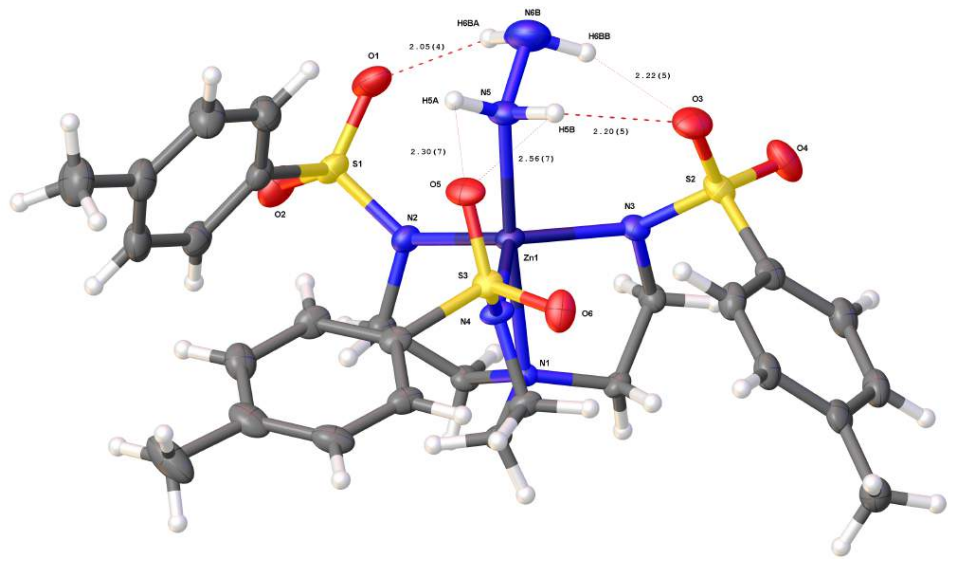

Experimental. Single colourless needle-shaped crystals of (CMW04-088BuZnTsN2H4) were recrystallised from a mixture of DCM and ethanol by vapor diffusion. A suitable crystal $(0.56 \times 0.20 \times 0.15) \mathrm{mm}$ was selected and mounted on a loop with paratone oil on a Bruker APEX-II CCD diffractometer. The crystal was cooled to $T=$ 100(2) K during data collection. The structure was solved with the ShelXT-2014/4 (Sheldrick, 2015) structure solution program in the space group P1 (\#1) using combined Patterson, direct and dual-space recycling methods (which confirmed the space group to be P-1 (\#2)) using Olex2 (Dolomanov et al., 2009). The structure was refined with version 2014/7 of ShelXL (Sheldrick, 2008) using Least Squares minimisation.

Crystal Data. $\mathrm{C}_{43} \mathrm{H}_{73} \mathrm{~N}_{7} \mathrm{O}_{6} \mathrm{~S}_{3} \mathrm{Zn}, M_{r}=945.63$, triclinic, P1 (No. 2), $\mathrm{a}=$ 9.117(2), $\mathrm{b}=$ 13.492(3) $\AA, \quad \mathrm{c}=$ 20.272(4) $\AA, \quad \alpha=97.526(3)^{\circ}, \quad \beta=96.225(3)^{\circ}, \quad \gamma=$ 109.087(3) $)^{\circ}, V=2305.5(9) \AA^{3}, T=100(2) \mathrm{K}, Z=2, Z^{\prime}=$ $1, \mu\left(\mathrm{MoK}_{a}\right)=0.722,33079$ reflections measured, 10558 unique $\left(R_{\text {int }} \#=0.0497\right)$ which were used in all calculations. The final $w R_{2}$ was 0.1793 (all data) and $R_{1}$ was $0.0713(\mathrm{I}>2(\mathrm{I}))$.

\begin{tabular}{|c|c|}
\hline Compound & $\begin{array}{l}\text { CMW04- } \\
088 \mathrm{BuZnTsN} 2 \mathrm{H} 4\end{array}$ \\
\hline Formula & $\mathrm{C}_{43} \mathrm{H}_{73} \mathrm{~N}_{7} \mathrm{O}_{6} \mathrm{~S}_{3} \mathrm{Zn}$ \\
\hline$D_{\text {calc. }} / \mathrm{g} \mathrm{cm}^{-3}$ & 1.362 \\
\hline$\mu / \mathrm{mm}^{-1}$ & 0.722 \\
\hline Formula Weight & 945.63 \\
\hline Colour & colourless \\
\hline Shape & needle \\
\hline Max Size/mm & 0.56 \\
\hline Mid Size/mm & 0.20 \\
\hline Min Size/mm & 0.15 \\
\hline$T / \mathrm{K}$ & $100(2)$ \\
\hline Crystal System & triclinic \\
\hline Space Group & P-1 \\
\hline$a / \AA ̊$ & $9.117(2)$ \\
\hline$b / \AA ̊$ & $13.492(3)$ \\
\hline$c / \AA ̊$ & $20.272(4)$ \\
\hline$\alpha /^{\circ}$ & $97.526(3)$ \\
\hline$\beta /^{\circ}$ & $96.225(3)$ \\
\hline$\gamma /^{\circ}$ & $109.087(3)$ \\
\hline $\mathrm{V} / \AA^{3}$ & $2305.5(9)$ \\
\hline$Z$ & 2 \\
\hline$Z^{\prime}$ & 1 \\
\hline$\Theta_{\min } /^{\circ}$ & 1.760 \\
\hline$\Theta_{\max } /^{\circ}$ & 27.484 \\
\hline Measured Refl. & 33079 \\
\hline Independent Refl. & 10558 \\
\hline $\begin{array}{l}\text { Reflections I > } \\
2 \operatorname{sigma(I)}\end{array}$ & 8173 \\
\hline$R_{\text {int }}$ & 0.0497 \\
\hline Parameters & 636 \\
\hline Restraints & 319 \\
\hline Largest Peak & 1.452 \\
\hline Deepest Hole & -1.004 \\
\hline GooF & 1.042 \\
\hline$w R_{2}$ (all data) & 0.1793 \\
\hline$w R_{2}$ & 0.1624 \\
\hline$R_{1}$ (all data) & 0.0954 \\
\hline$R_{1}$ & 0.0713 \\
\hline CCDC Number & 1429661 \\
\hline
\end{tabular}


Structure Quality Indicators

Reflections:

Refinement:

Experimental Extended. A colourless needle-shaped crystal with dimensions $0.56 \times 0.20 \times 0.15 \mathrm{~mm}$ was mounted on a loop with paratone oil. Data were collected using a Bruker APEX-II CCD diffractometer equipped with an Oxford Cryosystems low-temperature apparatus operating at $T=100(2) \mathrm{K}$.

Data were measured using $\omega$ scans with $\mathrm{MoK}_{a}$ radiation (fine-focus sealed tube, $45 \mathrm{kV}, 35 \mathrm{~mA}$ ). The total number of runs and images was based on the strategy calculation from the program APEX2 (Bruker, 2014). The maximum resolution achieved was $\Theta=27.484^{\circ}$.

Unit cell indexing was performed by using the APEX2 (Bruker, 2014) software and refined using SAINT (Bruker, V8.34A, 2013) on 9800 reflections, 30\% of the observed reflections. Data reduction, scaling and absorption corrections were performed using SAINT (Bruker, V8.34A, 2013) and SADABS-2014/5 (Bruker, 2014) was used for absorption correction. The Ratio of minimum to maximum transmission is 0.8574 . The $\lambda / 2$ correction factor is 0.00150 . The final completeness is $99.8 \%$ out to 27.484 in $\Theta$. The absorption coefficient $(\mu)$ of this material is $0.722 \mathrm{~mm}^{-1}$ and the minimum and maximum transmissions are 0.6393 and 0.7456 .

The structure was solved with ShelXT-2014/4 (Sheldrick, 2015) in the in the space group P1 (\#1) using combined Patterson, direct and dual-space recycling methods (which confirmed the space group to be P-1 (\#2)) and by using 0lex2 (Dolomanov et al., 2009) as the graphical interface. The structure was refined by Least Squares using version 2014/7 of ShelXL (Sheldrick, 2008). All non-hydrogen atoms were refined anisotropically. Hydrogen atom positions were calculated geometrically and refined using the riding model.

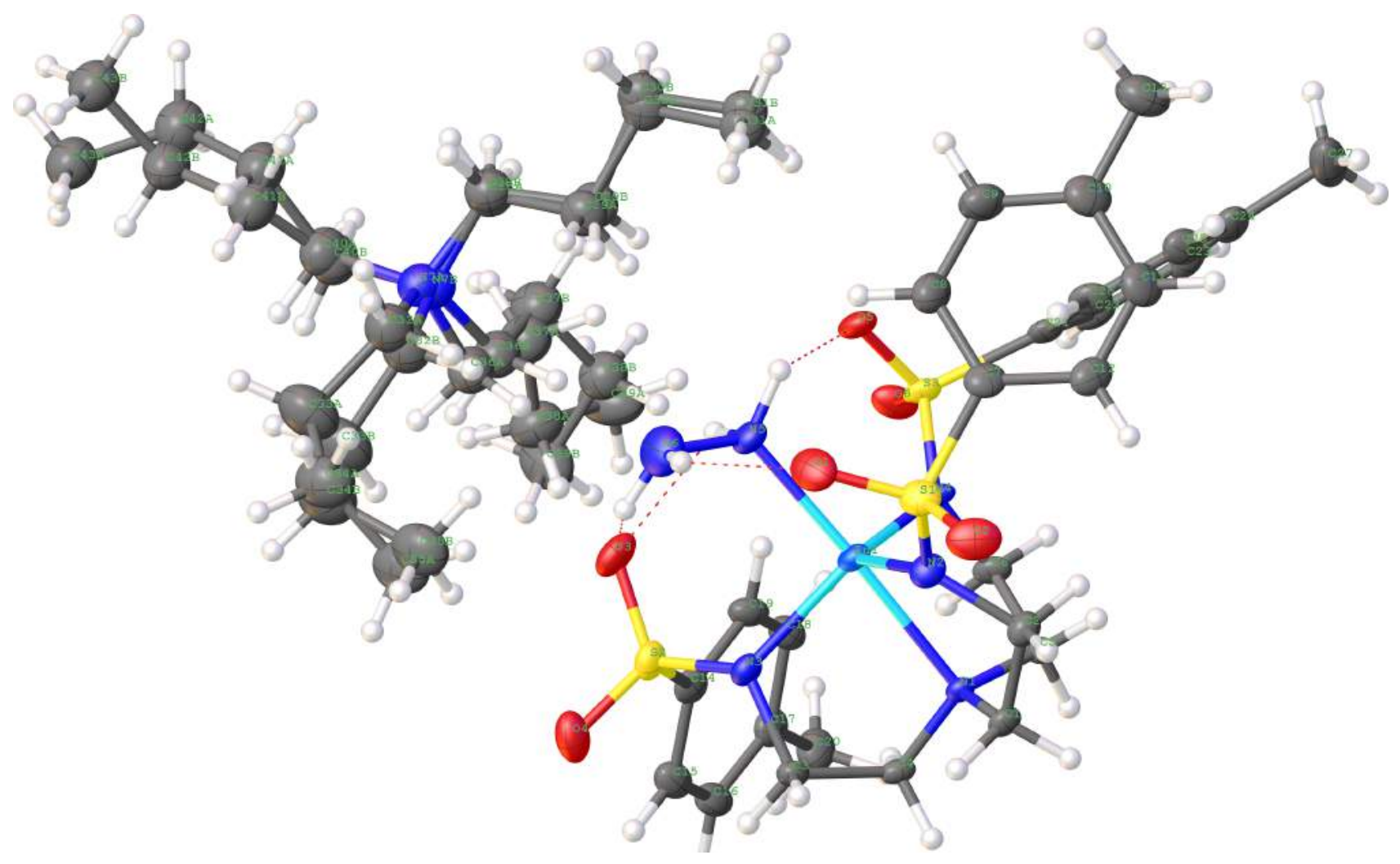

Figure 1: Plot of the asymmetric unit. 


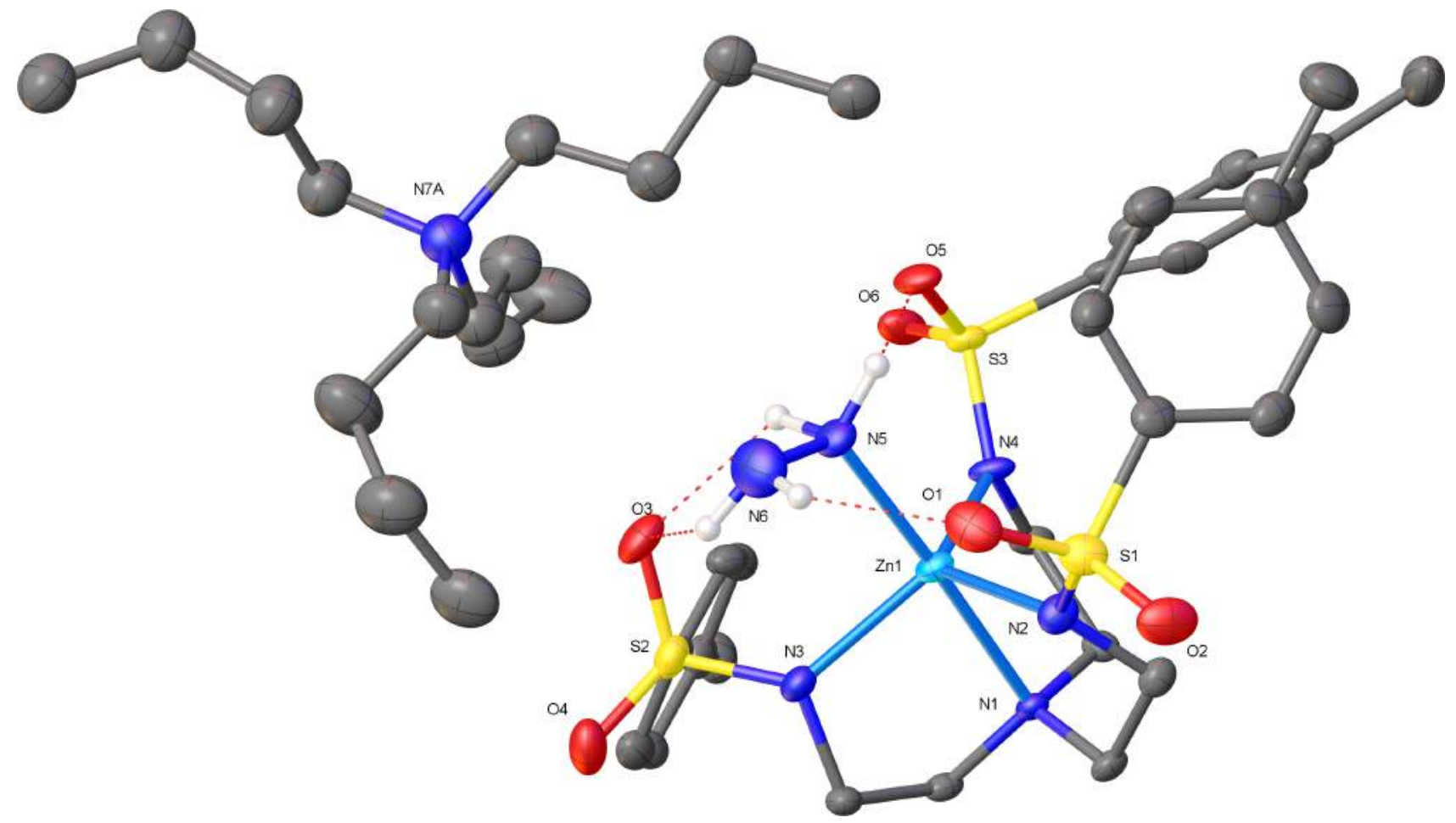

Figure 2: Plot of the asymmetric unit with one component of the disorder and $\mathrm{H}$ atoms (except $\mathrm{H}$ bonded $\mathrm{H}$ atoms) removed for clarity.

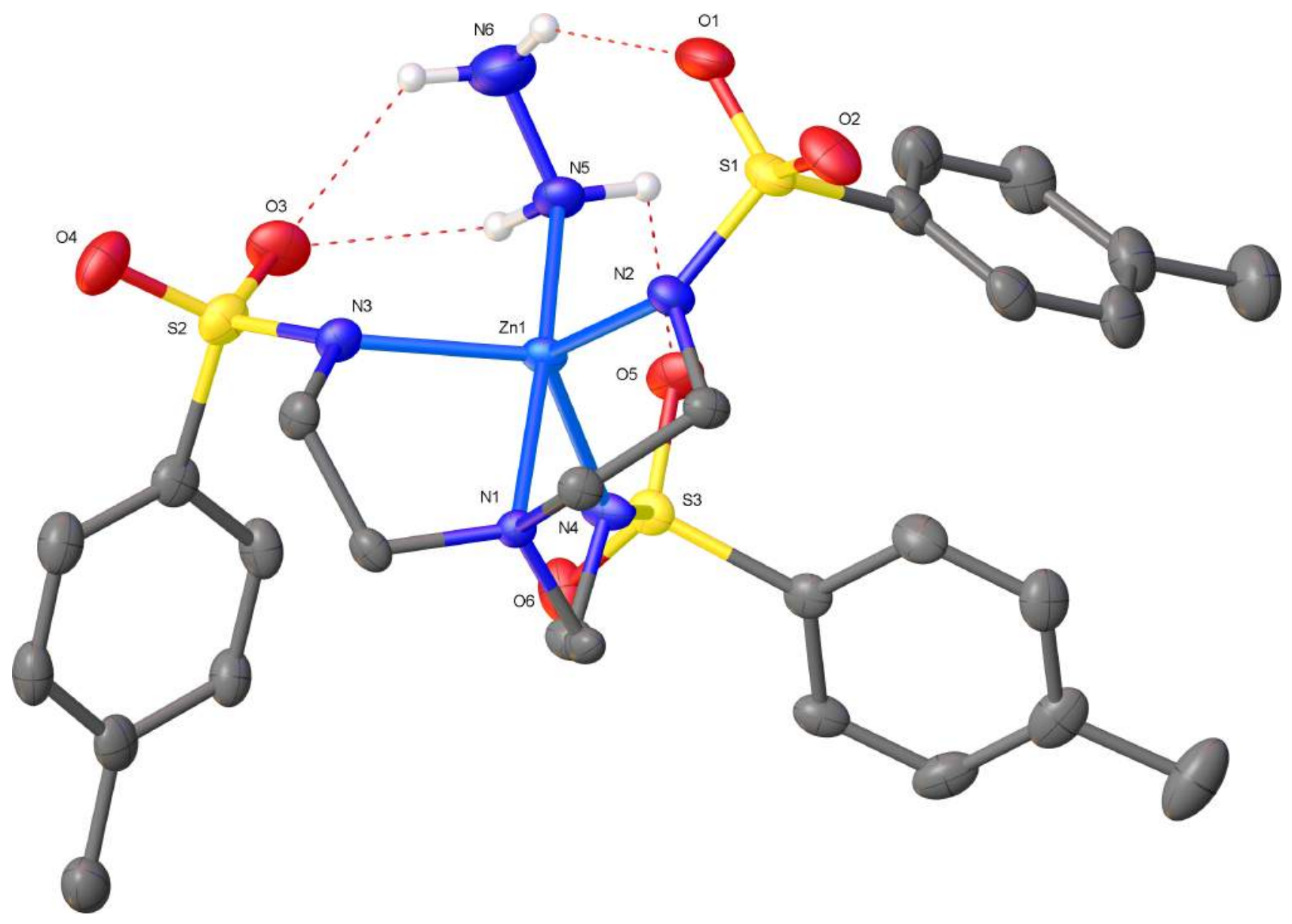


Figure 3:

Plot of the anionic part of the asymmetric unit.

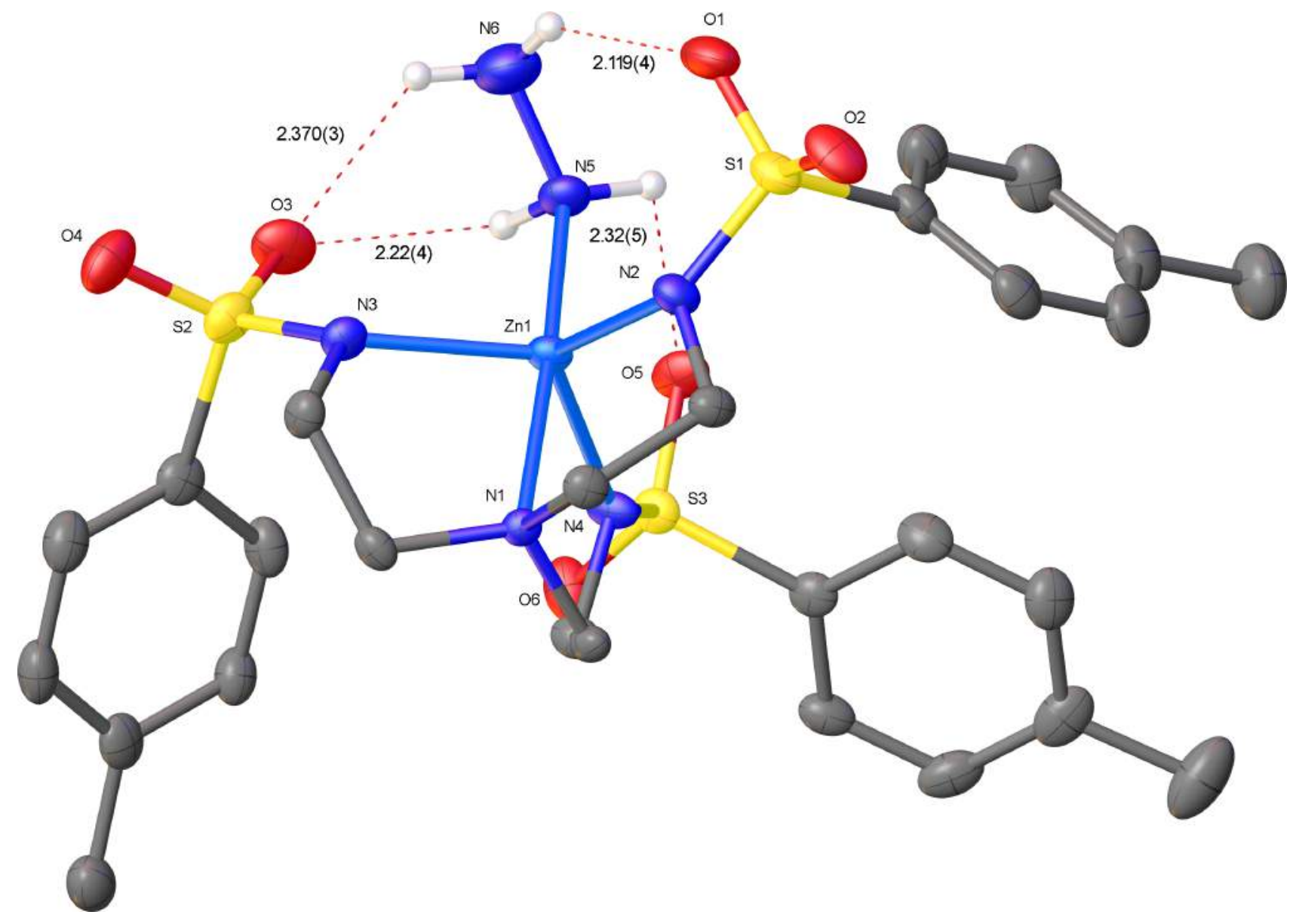

Figure 4:

Plot of the anionic part of the asymmetric unit with h-bond distances. 


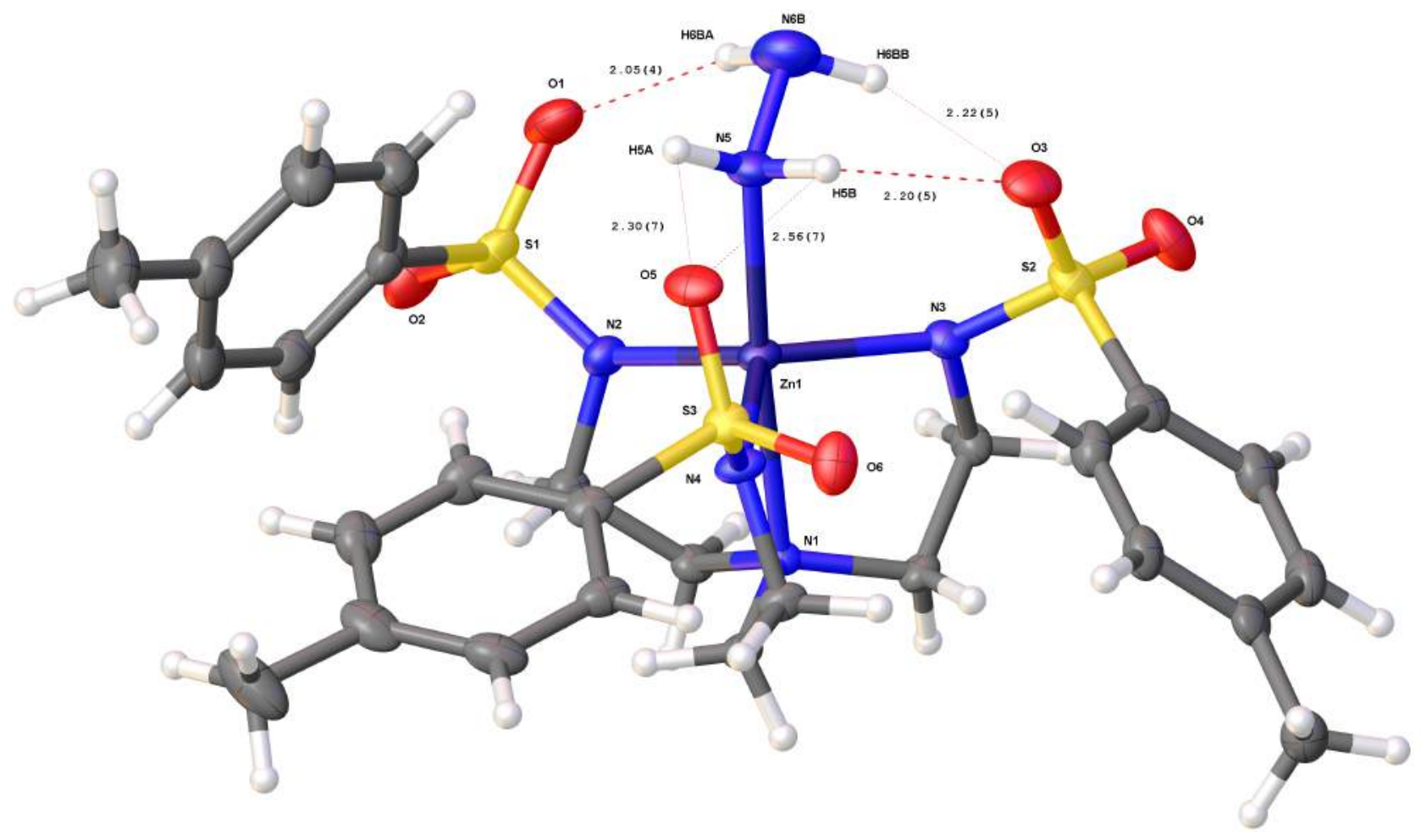

Figure 5: Plot of a second view of the anionic part of the asymmetric unit with h-bond distances.

\section{Data Plots: Diffraction Data}
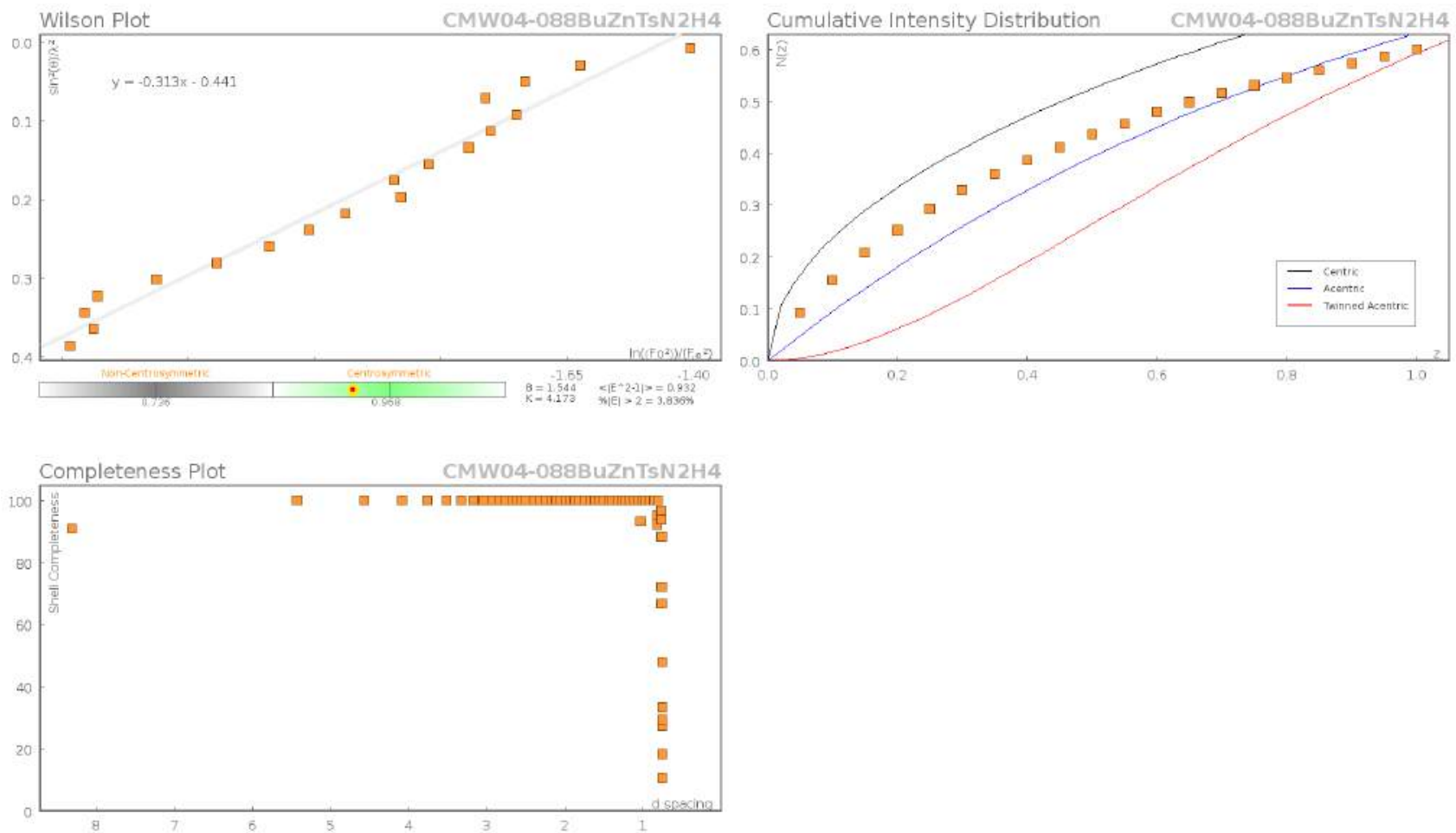
Data Plots: Refinement and Data
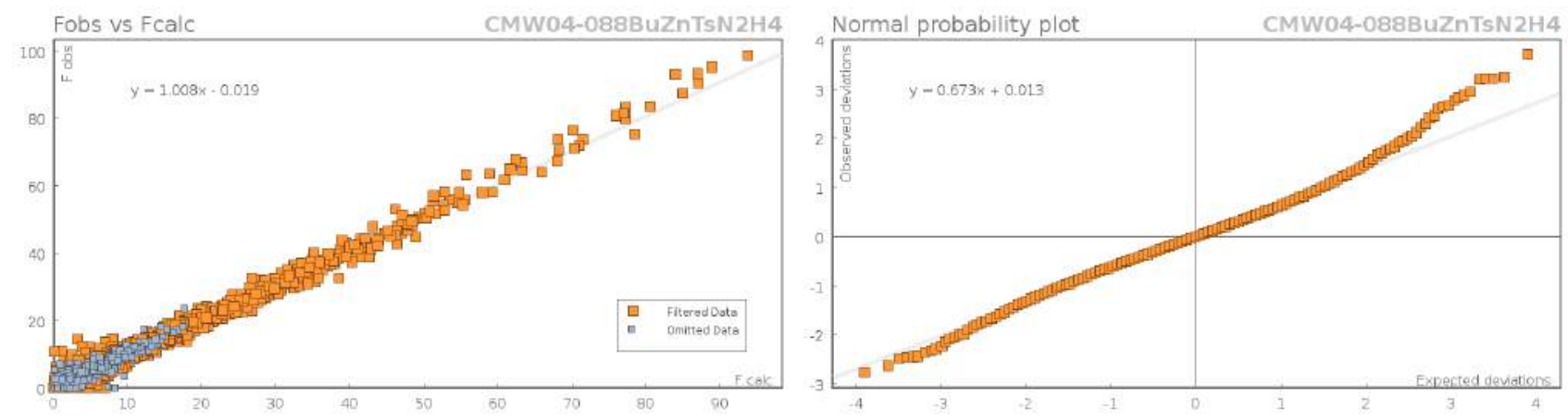

\section{Reflection Statistics}

\begin{tabular}{|c|c|c|c|}
\hline $\begin{array}{l}\text { Total reflections (after } \\
\text { filtering) }\end{array}$ & 33114 & Unique reflections & 10558 \\
\hline Completeness & 0.998 & Mean I/ $\sigma$ & 13.23 \\
\hline hklsub $>$ max $</$ sub $>$ collected & $(11,17,26)$ & hklsub $>\min </$ sub $>$ collected & $(-11,-17,-26)$ \\
\hline $\mathrm{hkl}_{\max }$ used & $(11,17,26)$ & $\mathrm{hkl}_{\min }$ used & $(-11,-17,0)$ \\
\hline Lim $d_{\max }$ collected & 20.0 & Lim $d_{\min }$ collected & 0.77 \\
\hline $\mathrm{d}_{\max }$ used & 11.57 & $\mathrm{~d}_{\min }$ used & 0.77 \\
\hline Friedel pairs & 10157 & Friedel pairs merged & 1 \\
\hline Inconsistent equivalents & 0 & $\mathrm{R}_{\text {int }}$ & 0.0497 \\
\hline $\mathrm{R}_{\text {sigma }}$ & 0.0547 & Intensity transformed & 0 \\
\hline Omitted reflections & 0 & Omitted by user (OMIT hkl) & 35 \\
\hline Multiplicity & $(10105,9472,1269,250,1)$ & Maximum mulitplicity & 9 \\
\hline Removed systematic absences & 0 & Filtered off (Shel/OMIT) & 747 \\
\hline
\end{tabular}

\section{Images of the Crystal on the Diffractometer}

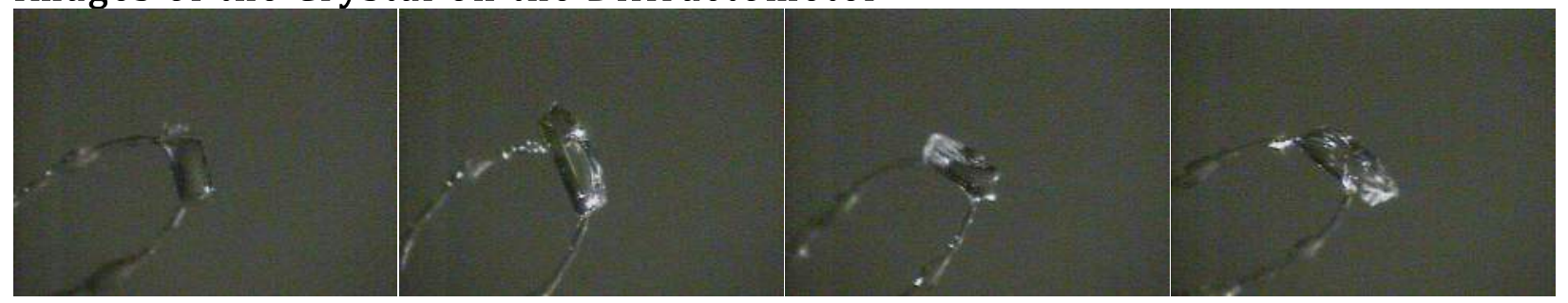

Table 1: Fractional Atomic Coordinates $\left(\times 10^{\wedge} 4\right)$ and Equivalent Isotropic Displacement Parameters $\left(\AA^{\wedge} 2 \times 10^{\wedge} 3\right)$ for CMW04-088BuZnTsN2H4. $U_{e q}$ is defined as $1 / 3$ of of the trace of the orthogonalised $U_{i j}$.

\begin{tabular}{lrrll}
\hline Atom & \multicolumn{1}{c}{$\mathbf{x}$} & $\mathbf{y}$ & \multicolumn{1}{c}{$\mathbf{z}$} & \multicolumn{1}{c}{$U_{e q}$} \\
\hline N7A & $890(11)$ & $-2351(7)$ & $3930(4)$ & $36.1(7)$ \\
C28A & $172(13)$ & $-1498(9)$ & $3891(7)$ & $38.8(9)$ \\
C29A & $1270(20)$ & $-441(12)$ & $3810(20)$ & $36.3(18)$ \\
C30A & $330(30)$ & $373(15)$ & $3830(30)$ & $35.7(11)$ \\
C31A & $1410(30)$ & $1419(14)$ & $3700(30)$ & $31.9(15)$ \\
C32A & $2275(15)$ & $-1979(9)$ & $4500(5)$ & $43.9(11)$ \\
C33A & $3030(17)$ & $-2780(12)$ & $4671(11)$ & $61.3(16)$ \\
C34A & $4784(15)$ & $-2209(19)$ & $4961(8)$ & $57(3)$ \\
C35A & $5890(20)$ & $-1990(20)$ & $4458(11)$ & $50.9(19)$ \\
C36A & $1448(12)$ & $-2642(12)$ & $3276(5)$ & $40.9(11)$
\end{tabular}




\begin{tabular}{|c|c|c|c|c|}
\hline Atom & $\mathbf{x}$ & $\mathrm{y}$ & $\mathbf{z}$ & $U_{e q}$ \\
\hline C37A & $390(20)$ & $-2798(18)$ & 2653(5) & 46.8(13) \\
\hline C38A & $660(20)$ & $-3495(14)$ & $2066(6)$ & $45(4)$ \\
\hline C39A & $-180(30)$ & $-3470(20)$ & 1393(6) & $60(6)$ \\
\hline C40A & $-342(16)$ & $-3356(7)$ & $4062(6)$ & $44.1(10)$ \\
\hline C41A & $-1100(20)$ & $-3289(10)$ & $4664(7)$ & $49.9(14)$ \\
\hline $\mathrm{C} 42 \mathrm{~A}$ & $-2527(15)$ & $-4259(13)$ & $4673(9)$ & $51(2)$ \\
\hline C43A & $-2370(20)$ & $-5099(12)$ & $5060(10)$ & 49.3(15) \\
\hline N7B & 881(5) & $-2318(3)$ & $3836(2)$ & $36.1(7)$ \\
\hline C28B & 196(7) & $-1445(4)$ & $3940(3)$ & $38.8(9)$ \\
\hline C29B & 1057(9) & $-444(6)$ & $3708(6)$ & $36.3(18)$ \\
\hline С30B & 194(13) & 391(7) & $3868(10)$ & $35.7(11)$ \\
\hline C31B & 1132(11) & $1427(6)$ & $3681(10)$ & $31.9(15)$ \\
\hline C32B & 2418(7) & $-2021(4)$ & 4314(3) & $43.9(11)$ \\
\hline C33B & $3213(9)$ & $-2844(6)$ & 4331(5) & 61.3(16) \\
\hline C34B & $4782(8)$ & $-2382(8)$ & $4821(5)$ & $60(2)$ \\
\hline C35B & 6091(9) & $-1542(7)$ & $4604(4)$ & $50.9(19)$ \\
\hline C36B & $1183(7)$ & $-2525(5)$ & $3116(2)$ & $40.9(11)$ \\
\hline C37B & $-135(10)$ & $-2748(7)$ & $2581(3)$ & $46.8(13)$ \\
\hline C38B & $262(9)$ & $-2901(5)$ & $1878(3)$ & $42.5(15)$ \\
\hline C39B & $617(11)$ & $-3901(6)$ & $1686(4)$ & $51.5(19)$ \\
\hline C40B & $-276(7)$ & $-3350(4)$ & $3972(3)$ & $44.1(10)$ \\
\hline C41B & $-657(9)$ & $-3408(5)$ & $4656(4)$ & $49.9(14)$ \\
\hline C42B & $-2018(9)$ & $-4396(5)$ & $4706(4)$ & $47.5(17)$ \\
\hline C43B & $-2798(9)$ & $-4434(6)$ & 5318(4) & 49.3(15) \\
\hline Zn1 & $6334.9(5)$ & 728.0(3) & $2309.7(2)$ & $17.10(12)$ \\
\hline S1 & $8062.5(12)$ & 2993.7(8) & $3351.1(5)$ & $24.9(2)$ \\
\hline S2 & $5887.2(12)$ & $-1781.5(8)$ & $2360.8(5)$ & $25.0(2)$ \\
\hline S3 & 2911.1(10) & 467.0(8) & $1434.3(5)$ & $19.0(2)$ \\
\hline 01 & $7553(4)$ & $2403(3)$ & $3880.3(15)$ & $35.5(7)$ \\
\hline 02 & $9543(4)$ & $3879(3)$ & $3522.6(16)$ & $35.7(8)$ \\
\hline 04 & $6741(4)$ & $-2410(2)$ & 2631.8(17) & $36.4(8)$ \\
\hline 05 & $2514(3)$ & 591(2) & $2102.8(15)$ & $26.7(6)$ \\
\hline 06 & 1944(3) & $-507(2)$ & $980.3(16)$ & $28.8(7)$ \\
\hline N1 & 7871(3) & $895(2)$ & $1500.0(15)$ & $14.2(6)$ \\
\hline N2 & $8034(4)$ & 2192(3) & 2713.5(16) & 21.4(7) \\
\hline 03 & $4562(4)$ & $-1728(3)$ & 2681.4(16) & $33.7(7)$ \\
\hline N3 & $6967(4)$ & $-616(3)$ & 2340.2(16) & $19.9(7)$ \\
\hline N4 & $4723(4)$ & $667(3)$ & 1496.2(15) & 18.8(7) \\
\hline N5 & 4841(4) & $470(3)$ & 3038.1(17) & 23.9(7) \\
\hline N6 & $5343(5)$ & 241(3) & $3669(2)$ & $44.3(10)$ \\
\hline C1 & $9314(4)$ & $1810(3)$ & 1777.5(19) & $18.2(7)$ \\
\hline $\mathrm{C} 2$ & 8931(5) & $2672(3)$ & 2205.2(19) & 20.7(8) \\
\hline C3 & $8360(4)$ & $-537(3)$ & $2006(2)$ & 19.6(8) \\
\hline C4 & $8183(4)$ & $-111(3)$ & 1357.9(19) & 17.6(7) \\
\hline C5 & $6989(4)$ & $1102(3)$ & $910.7(18)$ & $17.9(7)$ \\
\hline C6 & $5238(4)$ & 488(3) & 872.1(19) & $20.0(8)$ \\
\hline C7 & $6625(5)$ & $3597(3)$ & $3164(2)$ & $25.2(9)$ \\
\hline C8 & $5186(6)$ & $3264(4)$ & $3384(2)$ & $32.5(10)$ \\
\hline C9 & $4122(6)$ & $3767(4)$ & $3259(3)$ & $36.5(11)$ \\
\hline C10 & $4454(6)$ & $4610(3)$ & 2914(3) & $34.5(10)$ \\
\hline C11 & $5887(6)$ & $4927(3)$ & 2681(2) & $33.3(10)$ \\
\hline C12 & 6961(5) & $4422(3)$ & $2800(2)$ & $28.0(9)$ \\
\hline C13 & $3292(6)$ & $5173(4)$ & 2793(3) & 45.4(13) \\
\hline C14 & $5096(4)$ & $-2436(3)$ & 1513(2) & $22.5(8)$ \\
\hline C15 & $5707(5)$ & $-3141(3)$ & 1188(2) & $27.8(9)$ \\
\hline C16 & 5146(5) & $-3565(3)$ & $512(2)$ & 29.1(9) \\
\hline C17 & $3980(5)$ & $-3312(3)$ & 150(2) & 26.3(9) \\
\hline C18 & $3368(5)$ & $-2611(3)$ & 489(2) & 24.7(8) \\
\hline
\end{tabular}




\begin{tabular}{lcccl}
\hline Atom & $\mathbf{x}$ & $\mathbf{y}$ & $\mathbf{z}$ & \multicolumn{1}{c}{$U_{\text {eq }}$} \\
\hline C19 & $3921(5)$ & $-2167(3)$ & $1162(2)$ & $25.8(9)$ \\
C20 & $3392(6)$ & $-3767(4)$ & $-588(2)$ & $33.3(10)$ \\
C21 & $2566(4)$ & $1507(3)$ & $1060.8(19)$ & $18.8(7)$ \\
C22 & $1642(4)$ & $1289(3)$ & $436(2)$ & $22.6(8)$ \\
C23 & $1270(5)$ & $2082(4)$ & $167(2)$ & $29.2(9)$ \\
C24 & $1846(5)$ & $3115(4)$ & $514(2)$ & $31.4(10)$ \\
C25 & $2841(5)$ & $3346(3)$ & $1131(2)$ & $28.2(9)$ \\
C26 & $3198(4)$ & $2549(3)$ & $1406(2)$ & $24.1(8)$ \\
C27 & $1393(6)$ & $3982(5)$ & $239(3)$ & $49.9(15)$
\end{tabular}

Table 2: Anisotropic Displacement Parameters $\left(\times 10^{\wedge} 4\right)$ CMW04-088BuZnTsN2H4. The anisotropic displacement factor exponent takes the form: $-2 \mathrm{PI}^{\wedge} 2\left[a^{* \wedge} 2 \times U_{11}+\ldots 2 h k a^{*} \times b^{*} \times U_{12}\right]$

\begin{tabular}{|c|c|c|c|c|c|c|}
\hline Atom & $U_{11}$ & $U_{22}$ & $U_{33}$ & $U_{23}$ & $U_{13}$ & $U_{12}$ \\
\hline$\overline{N 7 A}$ & $34.7(13)$ & $37.4(13)$ & $36.0(14)$ & $6(1)$ & $6.9(11)$ & $12.1(10)$ \\
\hline $\mathrm{C} 28 \mathrm{~A}$ & $38.0(17)$ & $40.4(13)$ & $38(2)$ & $4.9(12)$ & $6.4(15)$ & $13.9(12)$ \\
\hline C29A & $32(2)$ & $40.3(14)$ & $33(5)$ & $4.7(17)$ & $-1(3)$ & $11.4(15)$ \\
\hline $\mathrm{C} 30 \mathrm{~A}$ & $30(2)$ & $38.8(15)$ & $34(3)$ & $5.1(14)$ & $-1.7(19)$ & $9.1(14)$ \\
\hline C31A & $25(4)$ & $37.4(16)$ & $31(3)$ & $3.5(14)$ & $-2(4)$ & $10.0(17)$ \\
\hline C32A & $40.7(15)$ & $45.4(18)$ & $44.8(19)$ & $9.0(14)$ & $4.1(14)$ & $14.5(13)$ \\
\hline C33A & $57(2)$ & $60(2)$ & $70(3)$ & $9.1(19)$ & $-3.3(18)$ & $29.2(17)$ \\
\hline C34A & $55(2)$ & $73(7)$ & $45(4)$ & $7(4)$ & $1(2)$ & $29(2)$ \\
\hline C35A & $51(2)$ & $65(4)$ & $38(4)$ & $5(3)$ & $-6(2)$ & $28(2)$ \\
\hline C36A & $44(2)$ & $40(2)$ & $38.9(14)$ & $6.8(12)$ & 8.7(13) & $14.9(16)$ \\
\hline C37A & $48(3)$ & $54(3)$ & $38.6(16)$ & $5.8(14)$ & $7.0(15)$ & $19(2)$ \\
\hline C38A & $39(7)$ & $54(8)$ & $40(3)$ & $3(3)$ & $7(3)$ & $14(7)$ \\
\hline C39A & $62(12)$ & $86(14)$ & $40(3)$ & $0(3)$ & $5(3)$ & $43(12)$ \\
\hline C40A & $44.6(16)$ & $41.4(14)$ & $45.4(16)$ & $8.2(12)$ & $10.3(13)$ & $12.3(12)$ \\
\hline C41A & $52(2)$ & $48.9(18)$ & $48.6(16)$ & $11.4(12)$ & $14.0(14)$ & 15.1(13) \\
\hline $\mathrm{C} 42 \mathrm{~A}$ & $51(2)$ & $50(2)$ & $50(3)$ & 11.1(18) & $10.9(16)$ & $14.6(14)$ \\
\hline C43A & $49(3)$ & $49(2)$ & $48(3)$ & $10(2)$ & $12(2)$ & $14(2)$ \\
\hline N7B & $34.7(13)$ & $37.4(13)$ & $36.0(14)$ & $6(1)$ & $6.9(11)$ & $12.1(10)$ \\
\hline C28B & $38.0(17)$ & $40.4(13)$ & $38(2)$ & $4.9(12)$ & $6.4(15)$ & $13.9(12)$ \\
\hline C29B & $32(2)$ & $40.3(14)$ & $33(5)$ & $4.7(17)$ & $-1(3)$ & $11.4(15)$ \\
\hline C30B & $30(2)$ & $38.8(15)$ & $34(3)$ & $5.1(14)$ & $-1.7(19)$ & $9.1(14)$ \\
\hline C31B & $25(4)$ & $37.4(16)$ & $31(3)$ & $3.5(14)$ & $-2(4)$ & $10.0(17)$ \\
\hline C32B & $40.7(15)$ & 45.4(18) & $44.8(19)$ & $9.0(14)$ & 4.1(14) & $14.5(13)$ \\
\hline C33B & $57(2)$ & $60(2)$ & $70(3)$ & $9.1(19)$ & $-3.3(18)$ & $29.2(17)$ \\
\hline C34B & $52(2)$ & $71(4)$ & $62(4)$ & $15(3)$ & $1(2)$ & $28(2)$ \\
\hline C35B & $51(2)$ & $65(4)$ & $38(4)$ & $5(3)$ & $-6(2)$ & $28(2)$ \\
\hline C36B & $44(2)$ & $40(2)$ & $38.9(14)$ & $6.8(12)$ & 8.7(13) & $14.9(16)$ \\
\hline C37B & $48(3)$ & $54(3)$ & $38.6(16)$ & $5.8(14)$ & $7.0(15)$ & $19(2)$ \\
\hline C38B & $43(4)$ & $49(3)$ & $35(2)$ & $6.4(17)$ & $5.3(19)$ & $16(3)$ \\
\hline C39B & $66(5)$ & $60(4)$ & $31(3)$ & $4(2)$ & $-3(3)$ & $31(4)$ \\
\hline $\mathrm{C} 40 \mathrm{~B}$ & $44.6(16)$ & $41.4(14)$ & $45.4(16)$ & $8.2(12)$ & $10.3(13)$ & $12.3(12)$ \\
\hline C41B & $52(2)$ & $48.9(18)$ & $48.6(16)$ & $11.4(12)$ & $14.0(14)$ & $15.1(13)$ \\
\hline C42B & $53(3)$ & $43(3)$ & $52(3)$ & $13(2)$ & $18(2)$ & $19(2)$ \\
\hline C43B & $49(3)$ & $49(2)$ & $48(3)$ & $10(2)$ & $12(2)$ & $14(2)$ \\
\hline Zn1 & $13.3(2)$ & $22.3(2)$ & $15.2(2)$ & 2.91(16) & $4.60(16)$ & $5.10(17)$ \\
\hline S1 & 25.1(5) & 28.1(5) & $18.7(5)$ & $-3.3(4)$ & $0.3(4)$ & $9.3(4)$ \\
\hline S2 & 21.7(5) & $22.3(5)$ & $29.4(5)$ & $10.6(4)$ & $6.4(4)$ & $2.4(4)$ \\
\hline S3 & $10.4(4)$ & 24.3(5) & $22.8(5)$ & $3.2(4)$ & $4.5(3)$ & $6.4(4)$ \\
\hline 01 & $46(2)$ & $45.3(19)$ & $19.7(15)$ & $1.6(13)$ & $4.6(14)$ & $23.8(16)$ \\
\hline 02 & $26.5(16)$ & $35.9(18)$ & $34.1(17)$ & $-11.7(14)$ & $-3.6(13)$ & $6.3(14)$ \\
\hline 04 & $34.3(18)$ & 27.1(16) & 44.4(19) & $16.7(14)$ & $0.1(15)$ & $4.0(14)$ \\
\hline 05 & $19.3(14)$ & 41.4(17) & $27.0(15)$ & $12.0(13)$ & $12.2(12)$ & $15.5(13)$ \\
\hline 06 & $14.9(14)$ & $23.8(15)$ & 43.6(18) & $-0.2(13)$ & $-1.3(13)$ & $5.6(11)$ \\
\hline N1 & $9.4(14)$ & 17.1(14) & $15.8(14)$ & 3.8(11) & $2.9(11)$ & $3.6(11)$ \\
\hline
\end{tabular}




\begin{tabular}{llllrrr}
\hline Atom & \multicolumn{1}{c}{$U_{11}$} & \multicolumn{1}{c}{$U_{22}$} & \multicolumn{1}{c}{$U_{33}$} & $U_{23}$ & $U_{13}$ & $U_{12}$ \\
\hline N2 & $21.3(17)$ & $21.3(16)$ & $18.4(16)$ & $-0.1(13)$ & $3.4(13)$ & $4.6(13)$ \\
O3 & $27.7(16)$ & $34.9(17)$ & $34.2(17)$ & $9.4(14)$ & $15.0(14)$ & $0.8(13)$ \\
N3 & $16.2(16)$ & $21.7(16)$ & $21.5(16)$ & $6.7(13)$ & $6.1(13)$ & $4.1(13)$ \\
N4 & $9.8(14)$ & $31.4(17)$ & $15.4(15)$ & $1.1(13)$ & $2.8(12)$ & $8.2(13)$ \\
N5 & $23.4(17)$ & $30.6(18)$ & $20.6(16)$ & $8.0(13)$ & $9.9(13)$ & $10.1(15)$ \\
N6 & $54(3)$ & $55(3)$ & $29.6(19)$ & $14.2(18)$ & $10.1(18)$ & $23(2)$ \\
C1 & $12.0(17)$ & $19.7(18)$ & $21.0(18)$ & $3.9(14)$ & $4.6(14)$ & $2.1(14)$ \\
C2 & $19.6(19)$ & $20.2(18)$ & $19.1(18)$ & $2.6(14)$ & $3.7(15)$ & $2.9(15)$ \\
C3 & $15.1(18)$ & $20.9(18)$ & $26(2)$ & $6.8(15)$ & $6.6(15)$ & $8.6(15)$ \\
C4 & $12.5(17)$ & $18.4(17)$ & $20.8(18)$ & $-0.5(14)$ & $5.2(14)$ & $4.5(14)$ \\
C5 & $19.5(18)$ & $23.0(18)$ & $15.4(17)$ & $4.3(14)$ & $5.6(14)$ & $11.7(15)$ \\
C6 & $10.9(17)$ & $28(2)$ & $19.8(18)$ & $0.6(15)$ & $0.0(14)$ & $7.3(15)$ \\
C7 & $26(2)$ & $20.6(19)$ & $26(2)$ & $-4.6(15)$ & $5.0(17)$ & $7.0(16)$ \\
C8 & $34(2)$ & $26(2)$ & $39(3)$ & $7.5(18)$ & $13(2)$ & $8.8(19)$ \\
C9 & $25(2)$ & $34(2)$ & $51(3)$ & $7(2)$ & $13(2)$ & $8.6(19)$ \\
C10 & $33(2)$ & $23(2)$ & $44(3)$ & $-2.0(19)$ & $3(2)$ & $9.0(19)$ \\
C11 & $36(3)$ & $18(2)$ & $42(3)$ & $1.3(18)$ & $5(2)$ & $6.2(18)$ \\
C12 & $25(2)$ & $20.8(19)$ & $32(2)$ & $-2.0(17)$ & $7.0(18)$ & $2.0(16)$ \\
C13 & $40(3)$ & $40(3)$ & $61(4)$ & $5(2)$ & $6(3)$ & $21(2)$ \\
C14 & $14.3(18)$ & $19.4(18)$ & $30(2)$ & $6.4(16)$ & $4.4(16)$ & $-0.6(15)$ \\
C15 & $18(2)$ & $21.2(19)$ & $42(3)$ & $8.3(18)$ & $3.4(18)$ & $4.3(16)$ \\
C16 & $26(2)$ & $19.7(19)$ & $45(3)$ & $8.0(18)$ & $12.3(19)$ & $10.0(17)$ \\
C17 & $25(2)$ & $15.9(18)$ & $35(2)$ & $6.6(16)$ & $9.3(18)$ & $1.9(16)$ \\
C18 & $18.2(19)$ & $18.7(18)$ & $36(2)$ & $5.5(16)$ & $3.0(17)$ & $5.5(15)$ \\
C19 & $17.1(19)$ & $21.4(19)$ & $40(2)$ & $3.6(17)$ & $8.5(17)$ & $7.4(16)$ \\
C20 & $41(3)$ & $25(2)$ & $35(2)$ & $6.6(18)$ & $11(2)$ & $12(2)$ \\
C21 & $8.8(15)$ & $25.3(18)$ & $22.0(18)$ & $3.3(14)$ & $4.5(13)$ & $5.2(14)$ \\
C22 & $13.8(18)$ & $29(2)$ & $19.7(19)$ & $-0.4(15)$ & $3.7(15)$ & $1.8(15)$ \\
C23 & $17(2)$ & $45(3)$ & $26(2)$ & $15.3(19)$ & $5.3(16)$ & $6.6(18)$ \\
C24 & $17(2)$ & $40(3)$ & $42(3)$ & $21(2)$ & $11.2(18)$ & $8.5(18)$ \\
C25 & $18(2)$ & $22(2)$ & $42(3)$ & $6.5(18)$ & $6.7(18)$ & $2.5(16)$ \\
C26 & $11.0(17)$ & $31(2)$ & $25(2)$ & $-0.3(16)$ & $-2.1(15)$ & $4.9(16)$ \\
C27 & $30(3)$ & $52(3)$ & $77(4)$ & $41(3)$ & $12(3)$ & $14(2)$ \\
& & & & &
\end{tabular}

Table 3: Bond Lengths in Å for CMW04-088BuZnTsN2H4.

\begin{tabular}{lll}
\hline Atom & Atom & Length $/ \AA$ \\
\hline N7A & C28A & $1.504(4)$ \\
N7A & C32A & $1.517(4)$ \\
N7A & C36A & $1.520(4)$ \\
N7A & C40A & $1.528(4)$ \\
C28A & C29A & $1.488(7)$ \\
C29A & C30A & $1.592(8)$ \\
C30A & C31A & $1.501(5)$ \\
C32A & C33A & $1.515(4)$ \\
C33A & C34A & $1.539(10)$ \\
C34A & C35A & $1.504(6)$ \\
C36A & C37A & $1.452(9)$ \\
C37A & C38A & $1.512(9)$ \\
C38A & C39A & $1.500(5)$ \\
C40A & C41A & $1.472(8)$ \\
C41A & C42A & $1.519(5)$ \\
C42A & C43A & $1.497(5)$ \\
N7B & C28B & $1.506(4)$ \\
N7B & C32B & $1.516(4)$ \\
N7B & C36B & $1.521(4)$ \\
N7B & C40B & $1.526(4)$ \\
C28B & C29B & $1.489(7)$
\end{tabular}

\begin{tabular}{lll}
\hline Atom & Atom & Length $\AA$ \\
\hline C29B & C30B & $1.592(8)$ \\
C30B & C31B & $1.501(5)$ \\
C32B & C33B & $1.513(5)$ \\
C33B & C34B & $1.539(10)$ \\
C34B & C35B & $1.505(6)$ \\
C36B & C37B & $1.453(9)$ \\
C37B & C38B & $1.512(9)$ \\
C38B & C39B & $1.500(6)$ \\
C40B & C41B & $1.471(8)$ \\
C41B & C42B & $1.519(4)$ \\
C42B & C43B & $1.496(6)$ \\
Zn1 & N1 & $2.258(3)$ \\
Zn1 & N2 & $2.067(3)$ \\
Zn1 & N3 & $2.080(3)$ \\
Zn1 & N4 & $2.060(3)$ \\
Zn1 & N5 & $2.102(3)$ \\
S1 & 01 & $1.446(3)$ \\
S1 & O2 & $1.451(3)$ \\
S1 & N2 & $1.565(3)$ \\
S1 & C7 & $1.788(4)$ \\
S2 & O4 & $1.444(3)$
\end{tabular}




\begin{tabular}{lll}
\hline Atom & Atom & Length $/ \AA$ \\
\hline S2 & O3 & $1.450(3)$ \\
S2 & N3 & $1.568(3)$ \\
S2 & C14 & $1.781(4)$ \\
S3 & O5 & $1.443(3)$ \\
S3 & O6 & $1.449(3)$ \\
S3 & N4 & $1.573(3)$ \\
S3 & C21 & $1.776(4)$ \\
N1 & C1 & $1.472(4)$ \\
N1 & C4 & $1.475(5)$ \\
N1 & C5 & $1.476(5)$ \\
N2 & C2 & $1.470(5)$ \\
N3 & C3 & $1.484(5)$ \\
N4 & C6 & $1.415(5)$ \\
N5 & N6 & $1.418(5)$ \\
C1 & C2 & $1.514(5)$ \\
C3 & C4 & $1.514(5)$ \\
C5 & C6 & $1.527(5)$ \\
C7 & C8 & $1.384(6)$ \\
C7 & C12 & $1.385(6)$
\end{tabular}

\begin{tabular}{lll}
\hline Atom & Atom & Length $/ \AA$ \\
\hline C8 & C9 & $1.374(7)$ \\
C9 & C10 & $1.380(7)$ \\
C10 & C11 & $1.391(7)$ \\
C10 & C13 & $1.509(7)$ \\
C11 & C12 & $1.382(6)$ \\
C14 & C15 & $1.382(6)$ \\
C14 & C19 & $1.395(6)$ \\
C15 & C16 & $1.386(6)$ \\
C16 & C17 & $1.384(6)$ \\
C17 & C18 & $1.392(6)$ \\
C17 & C20 & $1.508(6)$ \\
C18 & C19 & $1.382(6)$ \\
C21 & C22 & $1.382(5)$ \\
C21 & C26 & $1.387(6)$ \\
C22 & C23 & $1.380(6)$ \\
C23 & C24 & $1.378(7)$ \\
C24 & C25 & $1.397(6)$ \\
C24 & C27 & $1.512(6)$ \\
C25 & C26 & $1.386(6)$
\end{tabular}

Table 4: Bond Angles in ${ }^{\circ}$ for CMW04088BuZnTsN2H4.

\begin{tabular}{|c|c|c|c|}
\hline Atom & Atom & Atom & Angle $/^{\circ}$ \\
\hline C28A & N7A & C32A & 110.1(3) \\
\hline C28A & N7A & C36A & $111.8(4)$ \\
\hline C28A & N7A & C40A & 109.3(3) \\
\hline $\mathrm{C} 32 \mathrm{~A}$ & N7A & $\mathrm{C} 36 \mathrm{~A}$ & 108.8(3) \\
\hline C32A & N7A & C40A & $108.9(3)$ \\
\hline C36A & N7A & C40A & $107.9(3)$ \\
\hline C29A & C28A & N7A & $115.5(4)$ \\
\hline C28A & C29A & C30A & $108.5(5)$ \\
\hline C31A & C30A & C29A & $108.5(5)$ \\
\hline C33A & C32A & N7A & $117.7(5)$ \\
\hline C32A & C33A & C34A & $110.2(6)$ \\
\hline C35A & C34A & C33A & 116.5(7) \\
\hline C37A & C36A & N7A & 117.1(5) \\
\hline C36A & C37A & C38A & $114.1(6)$ \\
\hline C39A & C38A & C37A & $114.5(6)$ \\
\hline C41A & C40A & N7A & $119.0(4)$ \\
\hline C40A & C41A & C42A & $114.5(5)$ \\
\hline C43A & C42A & C41A & $119.9(6)$ \\
\hline C28B & N7B & C32B & $110.1(3)$ \\
\hline C28B & N7B & C36B & $111.6(4)$ \\
\hline C28B & N7B & C40B & $109.4(3)$ \\
\hline C32B & N7B & C36B & 108.8(3) \\
\hline C32B & N7B & C40B & 109.2(3) \\
\hline C36B & N7B & C40B & 107.7(3) \\
\hline C29B & C28B & N7B & $115.3(4)$ \\
\hline C28B & C29B & C30B & $108.5(5)$ \\
\hline C31B & C30B & C29B & $108.5(5)$ \\
\hline C33B & C32B & N7B & $117.9(4)$ \\
\hline C32B & C33B & C34B & $110.3(6)$ \\
\hline C35B & C34B & C33B & $116.4(7)$ \\
\hline C37B & C36B & N7B & $116.9(5)$ \\
\hline C36B & C37B & C38B & $114.2(6)$ \\
\hline C39B & C38B & C37B & $114.4(5)$ \\
\hline
\end{tabular}

\begin{tabular}{|c|c|c|c|}
\hline Atom & Atom & Atom & Angle $/^{\circ}$ \\
\hline$\overline{\mathrm{C} 41 \mathrm{~B}}$ & C40B & N7B & $119.2(4)$ \\
\hline $\mathrm{C} 40 \mathrm{~B}$ & C41B & C42B & $114.4(5)$ \\
\hline C43B & C42B & C41B & $120.0(6)$ \\
\hline N2 & Zn1 & N1 & $80.08(12)$ \\
\hline N2 & Zn1 & N3 & $117.35(13)$ \\
\hline N2 & Zn1 & N5 & $103.37(14)$ \\
\hline N3 & Zn1 & N1 & $80.35(12)$ \\
\hline N3 & Zn1 & N5 & $97.02(13)$ \\
\hline N4 & Zn1 & N1 & $80.14(11)$ \\
\hline N4 & Zn1 & N2 & $117.31(13)$ \\
\hline N4 & Zn1 & N3 & $116.81(13)$ \\
\hline N4 & Zn1 & N5 & $98.99(13)$ \\
\hline N5 & Zn1 & N1 & $176.40(13)$ \\
\hline 01 & S1 & 02 & $116.7(2)$ \\
\hline 01 & S1 & $\mathrm{N} 2$ & $108.79(19)$ \\
\hline 01 & S1 & C7 & $105.4(2)$ \\
\hline 02 & S1 & N2 & $111.92(19)$ \\
\hline 02 & S1 & C7 & $104.5(2)$ \\
\hline N2 & S1 & C7 & $109.06(19)$ \\
\hline 04 & $\mathrm{~S} 2$ & 03 & $115.9(2)$ \\
\hline 04 & S2 & N3 & $113.16(18)$ \\
\hline 04 & S2 & C14 & 105.1(2) \\
\hline 03 & S2 & N3 & $108.25(18)$ \\
\hline 03 & S2 & C14 & $106.28(19)$ \\
\hline N3 & S2 & C14 & $107.59(18)$ \\
\hline 05 & S3 & 06 & $115.74(19)$ \\
\hline 05 & S3 & N4 & $108.76(17)$ \\
\hline 05 & S3 & C21 & $106.10(18)$ \\
\hline 06 & S3 & N4 & $113.49(17)$ \\
\hline 06 & S3 & C21 & $104.96(18)$ \\
\hline N4 & S3 & C21 & $107.12(17)$ \\
\hline C1 & N1 & Zn1 & $106.3(2)$ \\
\hline $\mathrm{C} 1$ & N1 & C4 & $112.3(3)$ \\
\hline
\end{tabular}




\begin{tabular}{|c|c|c|c|c|c|c|c|}
\hline Atom & Atom & Atom & Angle $/^{\circ}$ & Atom & Atom & Atom & Angle $/^{\circ}$ \\
\hline $\mathrm{C} 1$ & N1 & $\mathrm{C} 5$ & $111.3(3)$ & C9 & C10 & C11 & $118.2(4)$ \\
\hline $\mathrm{C} 4$ & $\mathrm{~N} 1$ & Zn1 & $107.1(2)$ & C9 & C10 & $\mathrm{C} 13$ & $120.9(5)$ \\
\hline $\mathrm{C} 4$ & N1 & $\mathrm{C} 5$ & $112.6(3)$ & $\mathrm{C} 11$ & $\mathrm{C} 10$ & C13 & $121.0(5)$ \\
\hline C5 & N1 & Zn1 & $106.8(2)$ & C12 & C11 & C10 & $121.0(4)$ \\
\hline S1 & $\mathrm{N} 2$ & Zn1 & $128.2(2)$ & C11 & $\mathrm{C} 12$ & $\mathrm{C} 7$ & $119.9(4)$ \\
\hline $\mathrm{C} 2$ & N2 & Zn1 & $112.1(2)$ & C15 & C14 & S2 & $121.0(3)$ \\
\hline $\mathrm{C} 2$ & N2 & S1 & $115.2(3)$ & C15 & C14 & C19 & $120.0(4)$ \\
\hline S2 & N3 & $\mathrm{Zn} 1$ & $128.42(19)$ & C19 & C14 & $\mathrm{S} 2$ & $118.8(3)$ \\
\hline C3 & N3 & Zn1 & $110.9(2)$ & C14 & C15 & C16 & $119.2(4)$ \\
\hline C3 & N3 & S2 & $114.8(3)$ & C17 & C16 & C15 & $122.1(4)$ \\
\hline S3 & $\mathrm{N} 4$ & Zn1 & $130.58(18)$ & C16 & C17 & C18 & $117.8(4)$ \\
\hline C6 & $\mathrm{N} 4$ & Zn1 & $112.8(2)$ & C16 & C17 & $\mathrm{C} 20$ & $121.7(4)$ \\
\hline C6 & N4 & S3 & $114.1(2)$ & C18 & C17 & $\mathrm{C} 20$ & $120.5(4)$ \\
\hline N6 & N5 & Zn1 & $120.7(3)$ & C19 & C18 & C17 & $121.2(4)$ \\
\hline N1 & $\mathrm{C} 1$ & $\mathrm{C} 2$ & $110.4(3)$ & C18 & C19 & C14 & $119.7(4)$ \\
\hline N2 & $\mathrm{C} 2$ & $\mathrm{C} 1$ & $108.2(3)$ & $\mathrm{C} 22$ & $\mathrm{C} 21$ & S3 & $120.9(3)$ \\
\hline N3 & C3 & C4 & $109.6(3)$ & C22 & $\mathrm{C} 21$ & $\mathrm{C} 26$ & $119.3(4)$ \\
\hline N1 & $\mathrm{C} 4$ & C3 & $109.6(3)$ & $\mathrm{C} 26$ & $\mathrm{C} 21$ & S3 & 119.8(3) \\
\hline N1 & $\mathrm{C} 5$ & C6 & $109.0(3)$ & $\mathrm{C} 23$ & $\mathrm{C} 22$ & $\mathrm{C} 21$ & $121.1(4)$ \\
\hline N4 & C6 & $\mathrm{C} 5$ & $111.8(3)$ & C24 & $\mathrm{C} 23$ & $\mathrm{C} 22$ & $120.2(4)$ \\
\hline C8 & $\mathrm{C} 7$ & S1 & $121.2(3)$ & $\mathrm{C} 23$ & $\mathrm{C} 24$ & $\mathrm{C} 25$ & $118.8(4)$ \\
\hline C8 & C7 & C12 & $119.2(4)$ & C23 & $\mathrm{C} 24$ & $\mathrm{C} 27$ & $120.7(5)$ \\
\hline C12 & C7 & S1 & 119.5(3) & C25 & $\mathrm{C} 24$ & $\mathrm{C} 27$ & $120.5(5)$ \\
\hline C9 & $\mathrm{C} 8$ & $\mathrm{C} 7$ & $120.3(4)$ & $\mathrm{C} 26$ & $\mathrm{C} 25$ & $\mathrm{C} 24$ & $121.1(4)$ \\
\hline C8 & C9 & C10 & $121.3(4)$ & $\mathrm{C} 25$ & $\mathrm{C} 26$ & $\mathrm{C} 21$ & $119.4(4)$ \\
\hline
\end{tabular}

Table 5: Hydrogen Fractional Atomic Coordinates $\left(\times 10^{\wedge} 4\right)$ and Equivalent Isotropic Displacement Parameters $\left(\AA^{\wedge} 2 \times 10^{\wedge} 3\right)$ for CMW0 4-

$088 \mathrm{BuZnTsN} 2 \mathrm{H} 4 . U_{\text {eq }}$ is defined as $1 / 3$ of of the trace of the orthogonalised $U_{i j \text {. }}$

\begin{tabular}{|c|c|c|c|c|}
\hline Atom & $\mathbf{x}$ & $\mathrm{y}$ & & $U_{e q}$ \\
\hline $\mathrm{H} 28 \mathrm{~A}$ & -702 & -1750 & 3505 & 47 \\
\hline H28B & -286 & -1404 & 4306 & 47 \\
\hline $\mathrm{H} 29 \mathrm{~A}$ & 1672 & -503 & 3383 & 44 \\
\hline H29B & 2171 & -182 & 4187 & 44 \\
\hline $\mathrm{H} 30 \mathrm{~A}$ & -608 & 91 & 3472 & 43 \\
\hline H30B & -12 & 470 & 4269 & 43 \\
\hline $\mathrm{H} 31 \mathrm{~A}$ & 1514 & 1363 & 3226 & 48 \\
\hline H31B & 2444 & 1600 & 3978 & 48 \\
\hline H31C & 967 & 1976 & 3829 & 48 \\
\hline H32A & 3098 & -1366 & 4388 & 53 \\
\hline H32B & 1925 & -1713 & 4911 & 53 \\
\hline H33A & 2919 & -3301 & 4260 & 74 \\
\hline H33B & 2489 & -3174 & 5006 & 74 \\
\hline $\mathrm{H} 34 \mathrm{~A}$ & 4901 & -1523 & 5244 & 68 \\
\hline H34B & 5113 & -2647 & 5261 & 68 \\
\hline H35A & 5356 & -2407 & 4014 & 76 \\
\hline H35B & 6811 & -2179 & 4601 & 76 \\
\hline H35C & 6238 & -1226 & 4431 & 76 \\
\hline H36A & 2448 & -2072 & 3257 & 49 \\
\hline H36B & 1683 & -3305 & 3293 & 49 \\
\hline H37A & 497 & -2094 & 2530 & 56 \\
\hline H37B & -709 & -3124 & 2732 & 56 \\
\hline H38A & 1804 & -3266 & 2053 & 54 \\
\hline H38B & 318 & -4239 & 2146 & 54 \\
\hline
\end{tabular}




\begin{tabular}{|c|c|c|c|c|}
\hline Atom & $\mathrm{x}$ & $\mathrm{y}$ & $\mathrm{z}$ & $U_{e q}$ \\
\hline$\overline{\mathrm{H} 39 \mathrm{~A}}$ & 187 & -3849 & 1035 & 90 \\
\hline H39B & 51 & -2729 & 1332 & 90 \\
\hline H39C & -1311 & -3817 & 1371 & 90 \\
\hline $\mathrm{H} 40 \mathrm{~A}$ & -1184 & -3625 & 3664 & 53 \\
\hline H40B & 161 & -3901 & 4087 & 53 \\
\hline H41A & -1420 & -2653 & 4696 & 60 \\
\hline H41B & -314 & -3185 & 5069 & 60 \\
\hline $\mathrm{H} 42 \mathrm{~A}$ & -3330 & -3998 & 4849 & 61 \\
\hline H42B & -2958 & -4611 & 4198 & 61 \\
\hline $\mathrm{H} 43 \mathrm{~A}$ & -2297 & -5699 & 4750 & 74 \\
\hline H43B & -3283 & -5349 & 5285 & 74 \\
\hline $\mathrm{H} 43 \mathrm{C}$ & -1410 & -4801 & 5398 & 74 \\
\hline H28C & -902 & -1720 & 3701 & 47 \\
\hline H28D & 161 & -1272 & 4427 & 47 \\
\hline H29C & 1066 & -592 & 3217 & 44 \\
\hline H29D & 2159 & -151 & 3943 & 44 \\
\hline H30C & -882 & 121 & 3606 & 43 \\
\hline H30D & 114 & 498 & 4354 & 43 \\
\hline H31D & 1358 & 1295 & 3223 & 48 \\
\hline H31E & 2121 & 1753 & 3998 & 48 \\
\hline H31F & 527 & 1909 & 3701 & 48 \\
\hline $\mathrm{H} 32 \mathrm{C}$ & 3169 & -1375 & 4199 & 53 \\
\hline H32D & 2224 & -1823 & 4776 & 53 \\
\hline H33C & 3409 & -3064 & 3873 & 74 \\
\hline H33D & 2515 & -3481 & 4476 & 74 \\
\hline H34C & 4579 & -2074 & 5258 & 72 \\
\hline H34D & 5150 & -2977 & 4900 & 72 \\
\hline H35D & 6210 & -1795 & 4144 & 76 \\
\hline H35E & 7074 & -1397 & 4910 & 76 \\
\hline H35F & 5841 & -887 & 4616 & 76 \\
\hline H36C & 2046 & -1894 & 3047 & 49 \\
\hline H36D & 1562 & -3135 & 3069 & 49 \\
\hline H37C & -545 & -2152 & 2632 & 56 \\
\hline H37D & -985 & -3399 & 2632 & 56 \\
\hline H38C & -633 & -2911 & 1550 & 51 \\
\hline H38D & 1185 & -2282 & 1843 & 51 \\
\hline H39D & 662 & -4014 & 1202 & 77 \\
\hline H39E & -212 & -4509 & 1789 & 77 \\
\hline H39F & 1631 & -3834 & 1941 & 77 \\
\hline $\mathrm{H} 40 \mathrm{C}$ & -1275 & -3514 & 3663 & 53 \\
\hline H40D & 141 & -3927 & 3844 & 53 \\
\hline $\mathrm{H} 41 \mathrm{C}$ & -913 & -2770 & 4822 & 60 \\
\hline H41D & 288 & -3386 & 4958 & 60 \\
\hline $\mathrm{H} 42 \mathrm{C}$ & -2841 & -4524 & 4311 & 57 \\
\hline H42D & -1637 & -5006 & 4655 & 57 \\
\hline H43D & -3695 & -5101 & 5254 & 74 \\
\hline H43E & -3168 & -3828 & 5390 & 74 \\
\hline $\mathrm{H} 43 \mathrm{~F}$ & -2044 & -4396 & 5712 & 74 \\
\hline H5 & $4260(50)$ & $960(30)$ & $3100(30)$ & $52(17)$ \\
\hline H5A & $4050(40)$ & $-224(19)$ & $2840(30)$ & $56(17)$ \\
\hline H1A & 9828 & 2099 & 1404 & 22 \\
\hline H1B & 10056 & 1575 & 2055 & 22 \\
\hline $\mathrm{H} 2 \mathrm{~A}$ & 9913 & 3252 & 2429 & 25 \\
\hline H2B & 8301 & 2977 & 1919 & 25 \\
\hline H3A & 8458 & -1249 & 1905 & 23 \\
\hline H3B & 9325 & -55 & 2310 & 23 \\
\hline $\mathrm{H} 4 \mathrm{~A}$ & 9158 & 14 & 1158 & 21 \\
\hline H4B & 7304 & -641 & 1030 & 21 \\
\hline
\end{tabular}




\begin{tabular}{|c|c|c|c|c|c|}
\hline Atom & & & & & $U_{e q}$ \\
\hline$\overline{\mathrm{H} 5 \mathrm{~B}}$ & 7351 & 872 & 493 & 21 & \\
\hline $\mathrm{H} 5 \mathrm{C}$ & 7175 & 1875 & 955 & 21 & \\
\hline $\mathrm{H} 6 \mathrm{C}$ & 4629 & 710 & 522 & 24 & \\
\hline H6D & 5034 & -283 & 737 & 24 & \\
\hline H8 & 4932 & 2685 & 3622 & 39 & \\
\hline H9 & 3139 & 3530 & 3413 & 44 & \\
\hline H11 & 6131 & 5499 & 2437 & 40 & \\
\hline H12 & 7929 & 4641 & 2633 & 34 & \\
\hline $\mathrm{H} 13 \mathrm{~A}$ & 3861 & 5934 & 2814 & 68 & \\
\hline H13B & 2611 & 5082 & 3139 & 68 & \\
\hline H13C & 2648 & 4869 & 2347 & 68 & \\
\hline H15 & 6502 & -3332 & 1425 & 33 & \\
\hline H16 & 5575 & -4044 & 289 & 35 & \\
\hline H18 & 2555 & -2434 & 254 & 30 & \\
\hline H19 & 3502 & -1680 & 1383 & 31 & \\
\hline $\mathrm{H} 20 \mathrm{~A}$ & 2262 & -3896 & -686 & 50 & \\
\hline H20B & 3580 & -4440 & -697 & 50 & \\
\hline $\mathrm{H} 20 \mathrm{C}$ & 3951 & -3261 & -859 & 50 & \\
\hline H22 & 1256 & 583 & 188 & 27 & \\
\hline H23 & 614 & 1914 & -259 & 35 & \\
\hline H25 & 3279 & 4060 & 1366 & 34 & \\
\hline $\mathrm{H} 26$ & 3871 & 2715 & 1828 & 29 & \\
\hline $\mathrm{H} 27 \mathrm{~A}$ & 314 & 3905 & 303 & 75 & \\
\hline H27B & 1461 & 3921 & -243 & 75 & \\
\hline $\mathrm{H} 27 \mathrm{C}$ & 2111 & 4680 & 478 & 75 & \\
\hline H6A & 5721 & -325 & 3618 & 60 & \\
\hline H6B & 6172 & 817 & 3925 & 60 & \\
\hline
\end{tabular}

Table 6: Hydrogen Bond information for CMW04088BuZnTsN2 H4.

\begin{tabular}{lllcrrr}
\hline D & H & A & $\mathrm{d}(\mathrm{D}-\mathrm{H}) / \AA$ & $\mathrm{d}(\mathrm{H}-\mathrm{A}) / \AA$ & $\mathrm{d}(\mathrm{D}-\mathrm{A}) / \AA$ & $\mathrm{D}-\mathrm{H}-\mathrm{A} / \mathrm{deg}$ \\
\hline N5 & H5 & O5 & $0.973(5)$ & $2.32(5)$ & $2.749(5)$ & $106(4)$ \\
N5 & H5A & O3 & $0.974(5)$ & $2.22(4)$ & $2.878(5)$ & $124(4)$ \\
C2 & H2A & O2 & 0.99 & 2.37 & $2.835(5)$ & 108.1 \\
C3 & H3A & 04 & 0.99 & 2.56 & $2.997(5)$ & 106.4 \\
C8 & H8 & O1 & 0.95 & 2.55 & $2.915(6)$ & 103.0 \\
C12 & H12 & O2 & 0.95 & 2.72 & $2.981(6)$ & 96.8 \\
C15 & H15 & 04 & 0.95 & 2.55 & $2.909(6)$ & 102.8 \\
C19 & H19 & O3 & 0.95 & 2.72 & $3.015(6)$ & 98.8 \\
C22 & H22 & 06 & 0.95 & 2.48 & $2.870(5)$ & 104.7 \\
C26 & H26 & 05 & 0.95 & 2.88 & $3.074(5)$ & 92.6 \\
N6 & H6A & O3 & 0.93 & 2.37 & $2.938(5)$ & 119.0 \\
N6 & H6B & O1 & 0.93 & 2.12 & $2.897(6)$ & 140.2
\end{tabular}


Submitted by: Christian Wallen

Solved by: John Bacsa

Sample ID: CMW04_BuTsNH2OH

\section{Crystal Data and Experimental}

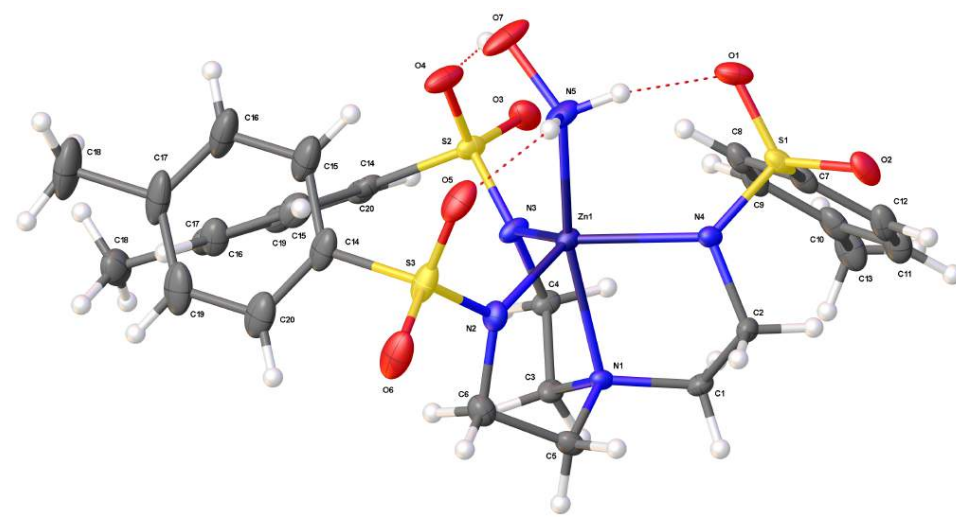

Experimental. Single colourless prism-shaped crystals of (CMW04-BuTsNH2OH) were recrystallised from a mixture of THF and diethyl ether by vapor diffusion. A suitable crystal $(0.63 \times 0.28 \times 0.20 \mathrm{~mm})$ was selected and mounted on a loop with paratone oil on a Bruker APEX-II CCD diffractometer. The crystal was cooled to $T=$ 100(2) K during the data collection. The structure was solved with Superflip (L. Palatinus \& G. Chapuis, 2007) using the Charge Flipping solution method and by using Olex2 (Dolomanov et al., 2009) as the graphical interface. The model was refined with version of ShelXL-97 (Sheldrick, 2008) using Least Squares minimisation.

Crystal Data. $\mathrm{C}_{43} \mathrm{H}_{72} \mathrm{~N}_{6} \mathrm{O}_{7} \mathrm{~S}_{3} \mathrm{Zn}, M_{r}=946.61$, triclinic, P-1 (No. 2), $a=13.579(2) \AA, b=18.615(3) \AA, c=20.015(4) \AA$, $\alpha=90.555(2)^{\circ}, \quad \beta=100.310(2)^{\circ}, \quad \gamma=110.958(2)^{\circ}, \quad V=$ 4633.2(14) $\AA^{3}, T=100(2) \mathrm{K}, Z=4, Z^{\prime}=2, \mu\left(\mathrm{MoK}_{a}\right)=0.720$, 77125 reflections measured, 21226 unique $\left(R_{\text {int }}=0.0402\right)$ which were used in all calculations. The final $w R_{2}$ was 0.1356 (all data) and $R_{1}$ was 0.0541 (I > 2(I)).

\section{Compound}

Formula

$D_{\text {calc. }} / \mathrm{g} \mathrm{cm}^{-3}$

$\mu / \mathrm{mm}^{-1}$

Formula Weight

Colour

Shape

Max Size/mm

Mid Size $/ \mathrm{mm}$

Min Size $/ \mathrm{mm}$

$T / \mathrm{K}$

Crystal System

Space Group

$a / \AA$

$b / \AA$

$c / \AA$

$\alpha /^{\circ}$

$\beta /^{\circ}$

$\gamma /{ }^{\circ}$

$\mathrm{V} / \AA^{3}$

Z

$Z^{\prime}$

$\Theta_{\min } /^{\circ}$

$\Theta_{\max } /^{\circ}$

Measured Refl.

Independent Refl.

Reflections Used

$R_{\text {int }}$

Parameters

Restraints

Largest Peak

Deepest Hole

GooF

$w R_{2}$ (all data)

$w R_{2}$

$R_{1}$ (all data)

$R_{1}$

CCDC Number

\section{CMWO4-BuTsNH2 \\ OH}

$\mathrm{C}_{43} \mathrm{H}_{72} \mathrm{~N}_{6} \mathrm{O}_{7} \mathrm{~S}_{3} \mathrm{Zn}$

1.357

0.720

946.61

colourless

prism

0.63

0.28

0.20

$100(2)$

triclinic

P-1

13.579(2)

18.615(3)

20.015(4)

90.555(2)

$100.310(2)$

110.958(2)

4633.2(14)

4

2

1.504

27.484

77125

21226

16159

0.0402

1335

606

1.174

$-0.417$

1.057

0.1356

0.1162

0.0795

0.0541

1429662

\section{Structure Quality Indicators}


A colourless prism-shaped crystal with dimensions $0.63 \times 0.28 \times 0.20 \mathrm{~mm}$ was mounted on a loop with paratone oil. Data were collected using a Bruker APEX-II CCD diffractometer equipped with an Oxford Cryosystems low-temperature apparatus operating at $T=100(2) \mathrm{K}$.

Data were measured using $\omega$ scans with a narrow frame width using $\mathrm{MoK}_{a}$ radiation (sealed tube, $45 \mathrm{kV}, 35$ $\mathrm{mA}$ ). The total number of runs and images was based on the strategy calculation from the program APEX2 (Bruker, 2014). The maximum resolution achieved was $\Theta=27.484^{\circ}$.

Unit cell indexing was performed by using the APEX2 (Bruker, 2014) software and refined using SAINT (Bruker, V8.34A, 2013) on 9762 reflections, 13\% of the observed reflections. Data reduction, scaling and absorption corrections were performed using SAINT (Bruker, V8.34A, 2013) and SADABS-2014/5 (Bruker,2014/5) was used for absorption correction.wR $R_{2}$ (int) was 0.1626 before and 0.0570 after correction.The Ratio of minimum to maximum transmission is 0.8518 . The $\lambda / 2$ correction factor is 0.00150 . The final completeness is $99.9 \%$ out to $27.484^{\circ}$ in $\Theta$. The absorption coefficient $(\mu)$ of this material is 0.720 $\mathrm{mm}^{-1}$ and the minimum and maximum transmissions are 0.6355 and 0.7461 .

The structure was solved with Superflip (L. Palatinus \& G. Chapuis, 2007) in the space group P-1 (\# 2) by Charge Flipping using the Charge Flipping solution method and by using 0lex2 (Dolomanov et al., 2009) as the graphical interface. The structure was refined by Least Squares using version of ShelXL-97 (Sheldrick, 2008). All non-hydrogen atoms were refined anisotropically. Hydrogen atom positions were calculated geometrically and refined using the riding model.

The value of $\mathrm{Z}^{\prime}$ is 2 . This means that there are two independent molecules in the asymmetric unit. There is considerable disorder in the crystal structure. The disorder was modeled using SAME commands to restrain the 1-2 and 1-3 interatomic distances within tolyl and $\mathrm{NEt}_{4}$ groups to be similar.

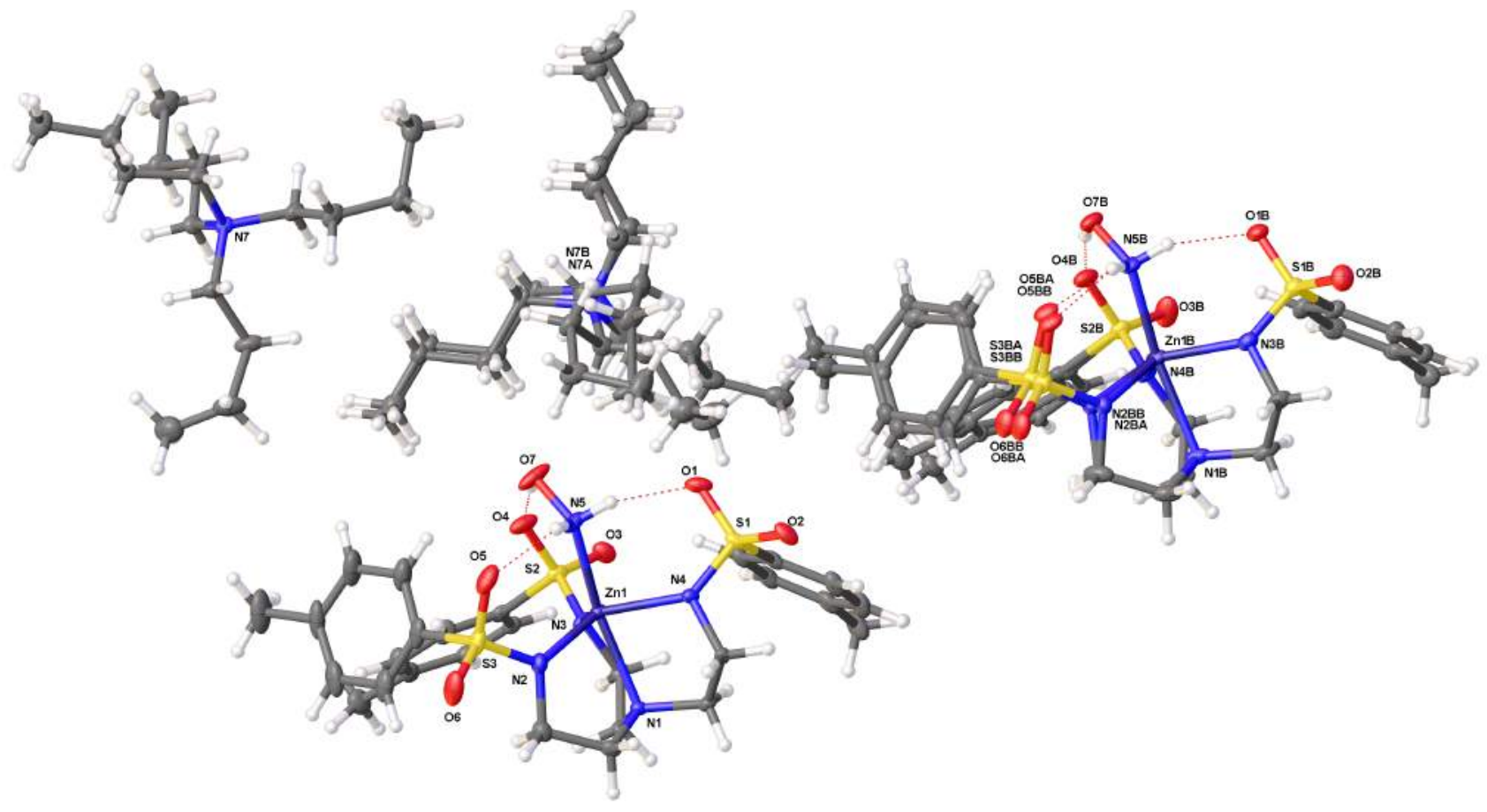

Figure 1: Plot of the asymmetric unit 


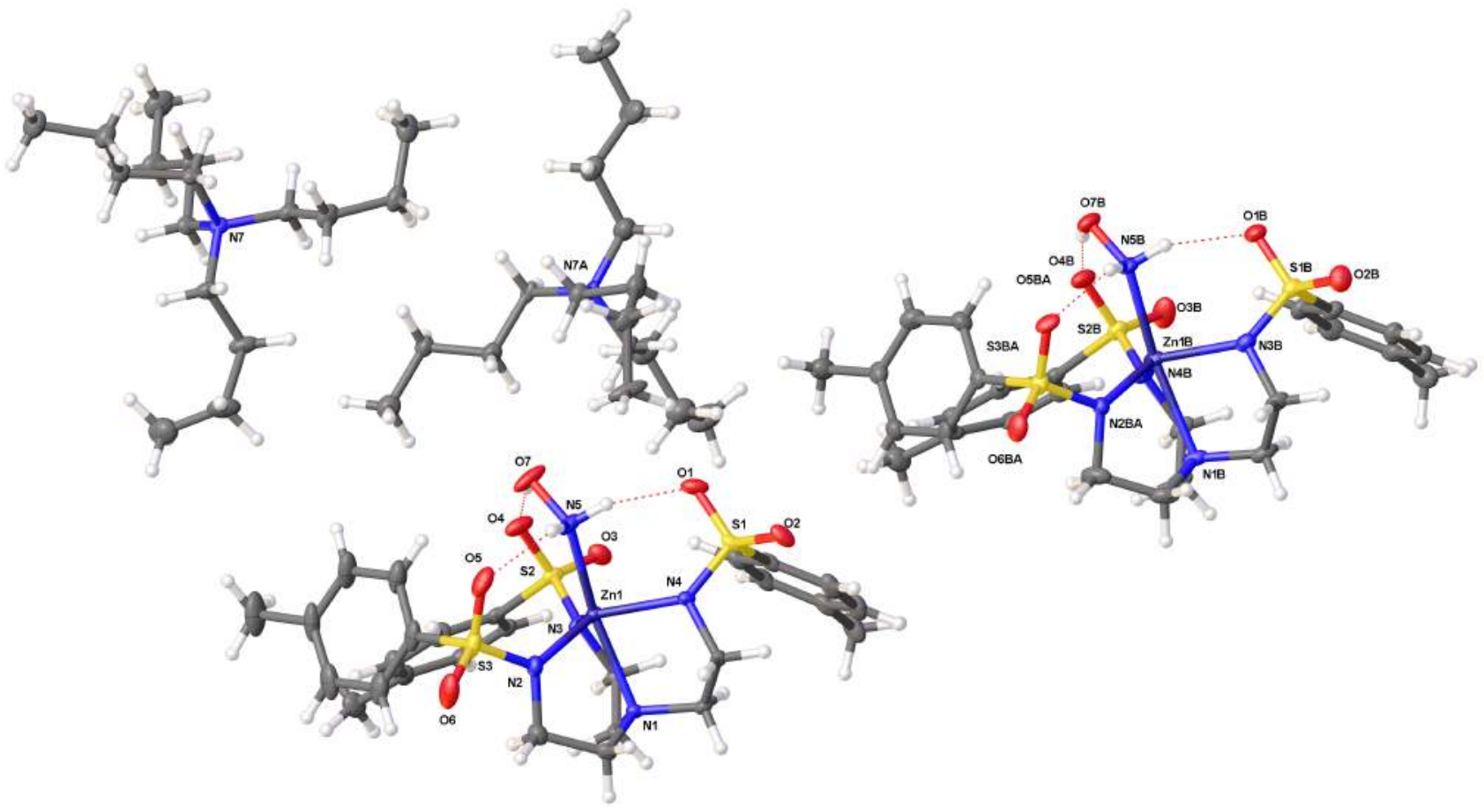

Figure 2: Plot of the asymmetric unit showing one component of the disorder only

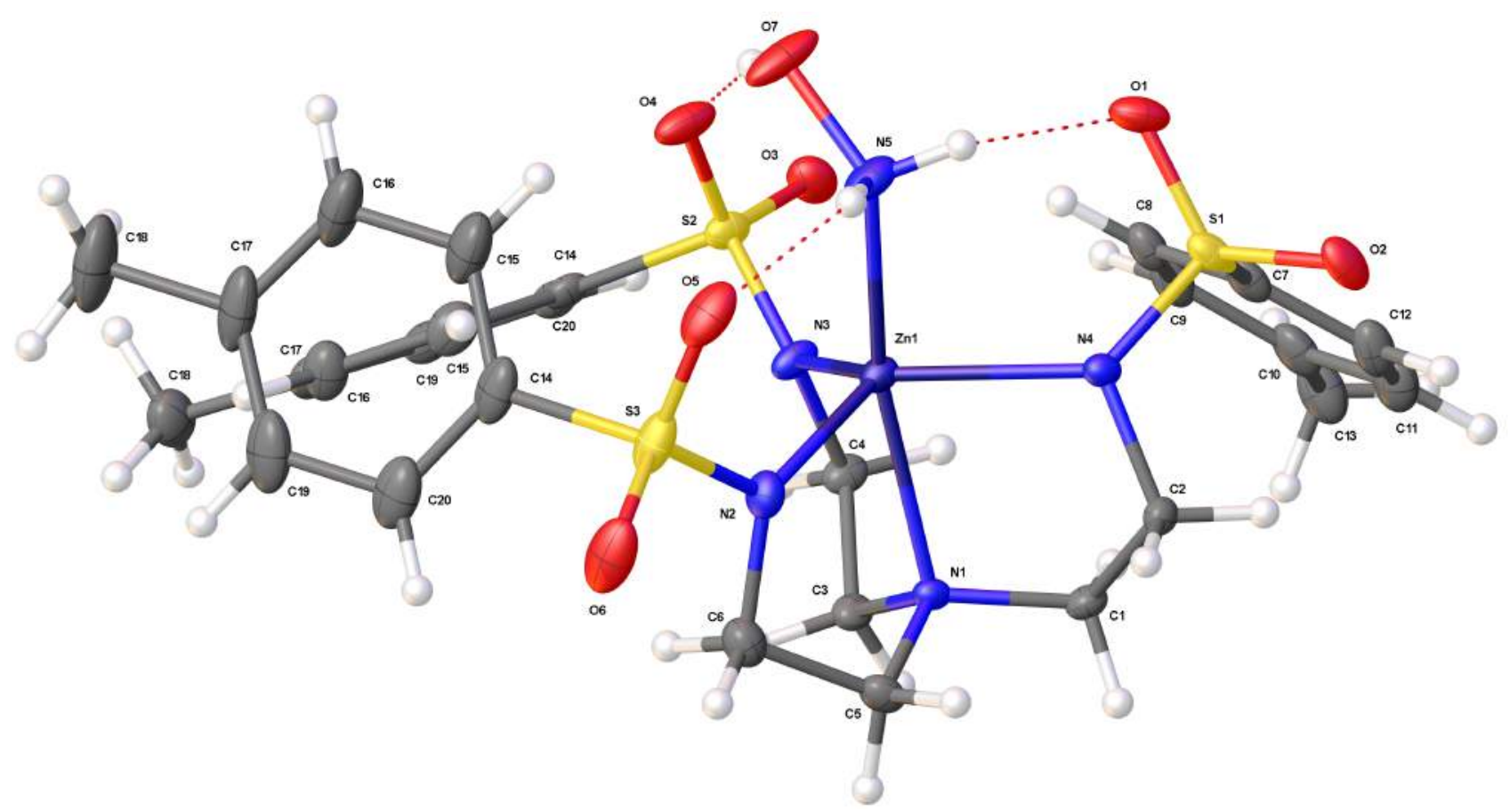

Figure 3: Plot of one of two symmetry independent [ZnR] anions. 
Data Plots: Diffraction Data
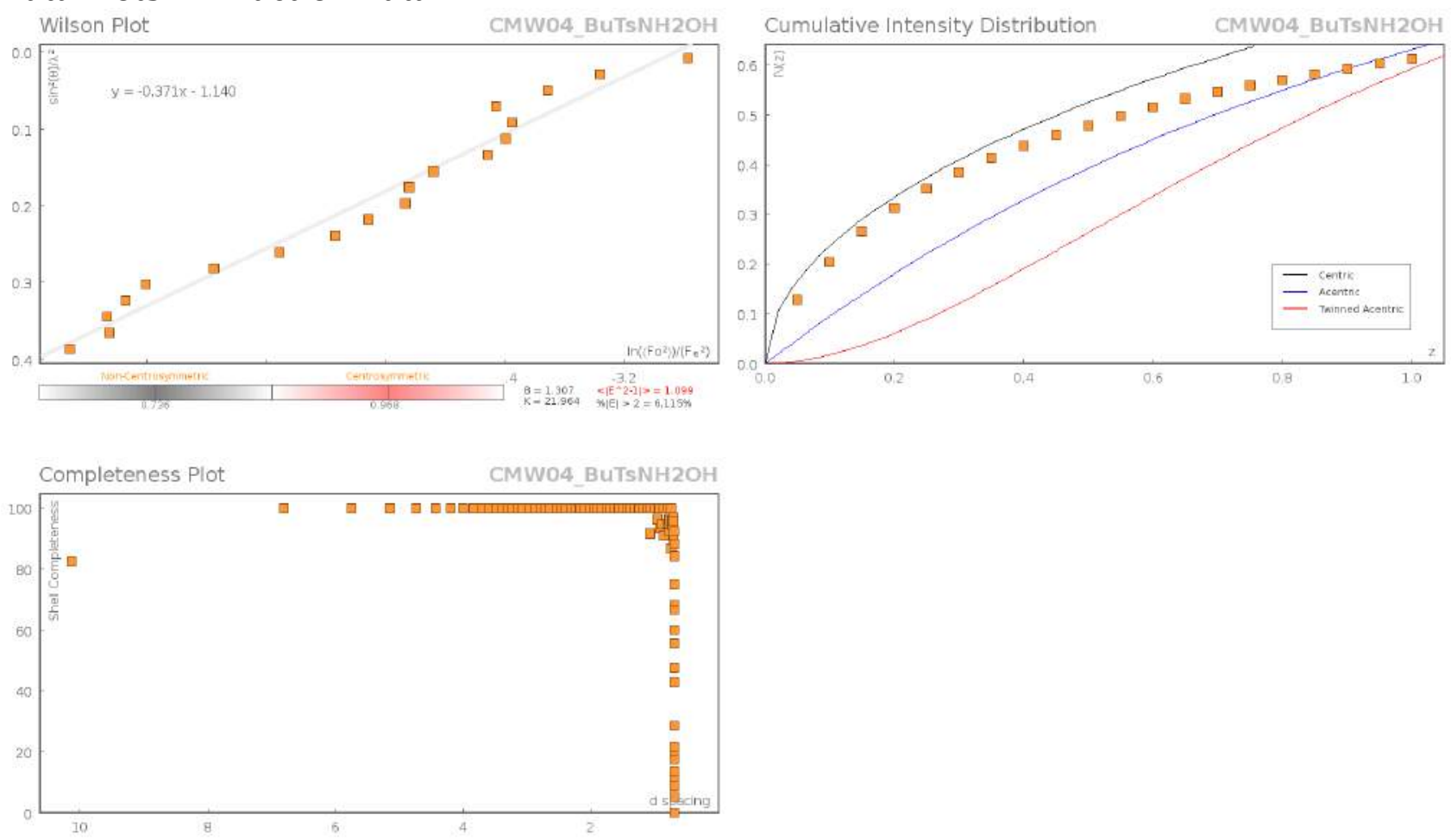

\section{Data Plots: Refinement and Data}
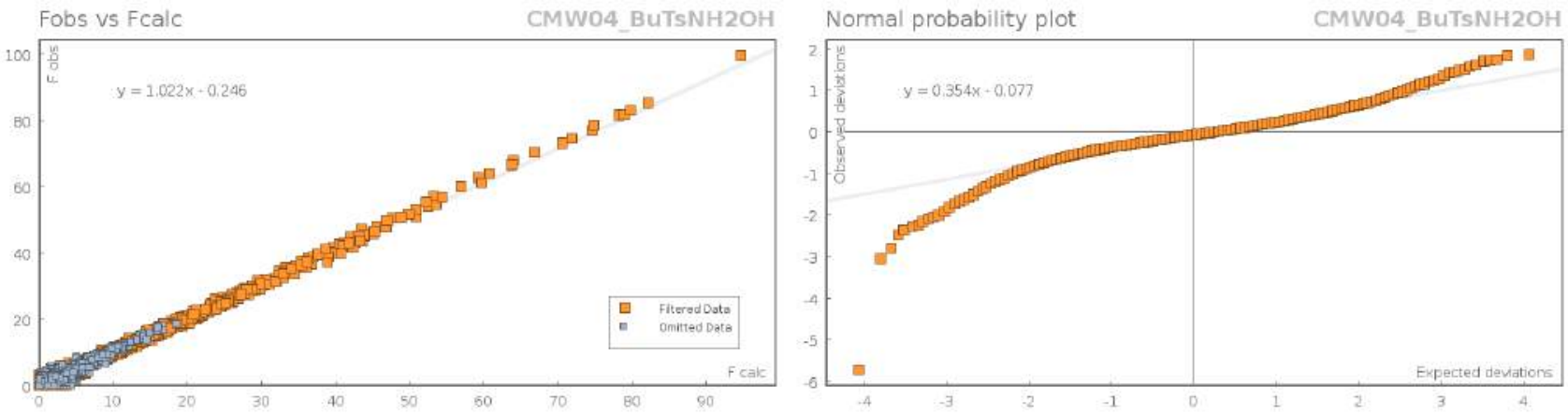

\section{Reflection Statistics}

Total reflections (after

filtering)

Completeness

hklsub $>$ max $</$ sub $>$ collected

hklmax used

Lim $d_{\max }$ collected

$\mathrm{d}_{\text {max }}$ used

Friedel pairs

Inconsistent equivalents

Rsigma

Omitted reflections

Multiplicity

Removed systematic absences 0
77126

0.999

$(19,26,28)$

$(17,24,25)$

20.0

13.54

25804

0

0.0382

0

(21271, 26911, 5225, 683, 368)
Unique reflections

21226

Mean I/ $\sigma$

15.03

hklsub $>$ min $</$ sub $>$ collected

hklmin used

Lim dmin collected

$(-19,-26,-28)$

$(-17,-24,0)$

$d_{\text {min }}$ used

0.77

Friedel pairs merged

0.77

Rint

0.0402

Intensity transformed

Omitted by user (OMIT hkl) 1

Maximum mulitplicity

10

Filtered off (Shel/OMIT) 
Table 1: Fractional Atomic Coordinates $\left(\times 10^{4}\right)$ and Equivalent Isotropic Displacement Parameters $\left(\AA^{2} \times 10^{3}\right)$ for CMW04_BuTsNH2OH. $U_{e q}$ is defined as $1 / 3$ of the trace of the orthogonalised $U_{i j}$.

\begin{tabular}{|c|c|c|c|c|}
\hline Atom & $\mathbf{x}$ & $\mathbf{y}$ & $\mathbf{z}$ & $U_{e q}$ \\
\hline$\overline{\mathrm{Zn} 1}$ & $6027.1(2)$ & $5517.1(2)$ & $2304.0(2)$ & $12.86(8)$ \\
\hline S1 & $4586.0(5)$ & $6633.2(4)$ & $2312.7(3)$ & $15.94(14)$ \\
\hline S2 & $4193.6(5)$ & $3892.6(4)$ & $1528.8(3)$ & $16.43(14)$ \\
\hline S3 & $8026.3(6)$ & $5261.2(4)$ & $3342.8(4)$ & $24.20(17)$ \\
\hline 01 & 3981.1(16) & $6038.7(12)$ & $2701.4(11)$ & $23.5(5)$ \\
\hline 02 & $4731.5(16)$ & $7419.2(12)$ & 2521.8(11) & $22.8(5)$ \\
\hline 03 & $3207.2(16)$ & $3891.0(12)$ & $1114.2(11)$ & $24.0(5)$ \\
\hline 04 & $4134.8(18)$ & $3696.1(12)$ & $2225.9(11)$ & $26.5(5)$ \\
\hline 05 & 7504.6(19) & $5359.3(12)$ & 3889.1(11) & $29.4(5)$ \\
\hline 06 & $9190.5(19)$ & $5535.9(13)$ & $3502.5(13)$ & $35.3(6)$ \\
\hline 07 & $4763(2)$ & $4550.1(13)$ & 3401.1(11) & $35.6(6)$ \\
\hline N2 & $7596.7(19)$ & $5611.9(13)$ & 2694.5(12) & $18.3(5)$ \\
\hline N1 & $6825.5(17)$ & $6069.5(12)$ & $1451.0(11)$ & $12.6(4)$ \\
\hline N3 & $5184.0(19)$ & $4665.9(13)$ & 1542.1(11) & $16.1(5)$ \\
\hline N5 & $5434(2)$ & $5283.9(14)$ & $3215.9(12)$ & $22.5(5)$ \\
\hline N4 & $5680.0(18)$ & $6533.3(12)$ & 2289.8(11) & $13.4(4)$ \\
\hline C7 & $3835(2)$ & $6461.9(16)$ & $1464.5(15)$ & $18.2(6)$ \\
\hline C8 & $3358(2)$ & $5712.3(17)$ & $1156.5(15)$ & $19.6(6)$ \\
\hline C12 & $3811(2)$ & 7080.4(17) & $1083.4(16)$ & $23.0(6)$ \\
\hline C1 & $6492(2)$ & $6725.9(15)$ & $1273.9(13)$ & $14.7(5)$ \\
\hline $\mathrm{C} 2$ & $6392(2)$ & $7115.2(15)$ & $1917.9(13)$ & $13.9(5)$ \\
\hline $\mathrm{C} 3$ & $6458(2)$ & $5461.9(15)$ & $887.3(13)$ & $15.4(5)$ \\
\hline C10 & $2834(2)$ & $6194.7(18)$ & $86.4(16)$ & $23.5(6)$ \\
\hline C4 & $5304(2)$ & 4933.7(15) & $860.5(13)$ & $15.8(5)$ \\
\hline C11 & $3312(2)$ & 6939.5(18) & $400.4(17)$ & $25.5(7)$ \\
\hline C6 & $8255(2)$ & $5744.5(17)$ & 2163.2(15) & $21.7(6)$ \\
\hline C13 & $2315(3)$ & $6059(2)$ & $-659.3(16)$ & $28.5(7)$ \\
\hline C5 & $7994(2)$ & $6322.7(16)$ & $1708.2(14)$ & $17.5(6)$ \\
\hline C9 & $2857(2)$ & $5585.3(17)$ & $479.9(16)$ & $22.3(6)$ \\
\hline Zn1B & $913.4(2)$ & 10371.4(2) & $2337.0(2)$ & 15.63(8) \\
\hline S1B & $-508.8(6)$ & $11492.9(4)$ & $2343.9(3)$ & $17.99(14)$ \\
\hline S2B & $-844.5(6)$ & $8796.9(4)$ & $1461.2(4)$ & $18.34(15)$ \\
\hline S3BA & 2872(7) & 10191(2) & $3419(4)$ & $17.8(5)$ \\
\hline S3BB & $2819(7)$ & $10028(2)$ & $3437(4)$ & $17.8(5)$ \\
\hline 01B & $-1155.8(17)$ & $10868.8(12)$ & $2686.5(10)$ & $22.5(4)$ \\
\hline O2B & $-365.8(18)$ & $12263.4(12)$ & $2590.7(11)$ & $26.4(5)$ \\
\hline O3B & $-1805.0(18)$ & 8791.6(13) & $1014.4(13)$ & $34.1(6)$ \\
\hline 04B & $-971.4(18)$ & $8556.5(12)$ & 2136.8(11) & $25.5(5)$ \\
\hline O5BA & 2354(14) & 10262(10) & $3936(11)$ & $20.4(17)$ \\
\hline 05BB & $2188(14)$ & 10141(10) & $3964(11)$ & $20.4(17)$ \\
\hline 06BA & $4020(12)$ & $10500(5)$ & $3612(8)$ & $25.5(14)$ \\
\hline O6BB & $3988(12)$ & $10293(5)$ & $3632(8)$ & $25.5(14)$ \\
\hline O7B & $-475.6(18)$ & $9308.2(12)$ & $3355.4(11)$ & $25.1(5)$ \\
\hline N1B & $1812.5(19)$ & $10985.2(14)$ & 1518.5(11) & 19.1(5) \\
\hline N2BA & $2444(10)$ & 10536(5) & 2763(5) & $15.4(11)$ \\
\hline N2BB & $2465(10)$ & 10386(5) & 2768(5) & $15.4(11)$ \\
\hline N3B & $584.0(19)$ & $11388.2(13)$ & $2338.0(12)$ & $18.1(5)$ \\
\hline N4B & 127.1(19) & $9583.6(13)$ & $1524.7(11)$ & $17.0(5)$ \\
\hline N5B & $214(2)$ & $10058.1(14)$ & $3213.2(12)$ & $20.2(5)$ \\
\hline $\mathrm{C} 2 \mathrm{~B}$ & $3159(2)$ & $10605(2)$ & $2257.6(15)$ & $27.6(7)$ \\
\hline C3B & $1502(2)$ & 11659.0(17) & $1364.7(14)$ & $22.2(6)$ \\
\hline C4B & $1346(2)$ & 12002.1(17) & 2015.1(15) & 21.8(6) \\
\hline C5B & $1474(2)$ & $10415.7(17)$ & $931.0(14)$ & $22.5(6)$ \\
\hline C1B & $2966(2)$ & $11212.5(19)$ & $1813.4(15)$ & $25.4(6)$ \\
\hline C6B & $300(2)$ & 9897.5(17) & $861.6(14)$ & $20.7(6)$ \\
\hline
\end{tabular}




\begin{tabular}{|c|c|c|c|c|}
\hline Atom & $\mathbf{x}$ & $\mathbf{y}$ & $\mathbf{z}$ & $U_{e q}$ \\
\hline$\overline{\mathrm{C} 7 \mathrm{~B}}$ & $-1213(2)$ & $11374.3(17)$ & $1487.0(14)$ & $19.6(6)$ \\
\hline C8B & $-1703(2)$ & 10633.8(17) & 1154.3(16) & $23.5(6)$ \\
\hline С9B & $-2200(2)$ & $10540.7(18)$ & 477.2(16) & $24.5(6)$ \\
\hline C10B & $-2218(2)$ & 11173.0(18) & 112.3(15) & $21.8(6)$ \\
\hline C11B & $-1720(2)$ & $11904.9(17)$ & $452.6(15)$ & $20.7(6)$ \\
\hline C12B & $-1225(2)$ & $12009.5(17)$ & $1134.9(15)$ & $20.9(6)$ \\
\hline C13B & $-2745(3)$ & $11072(2)$ & $-633.7(16)$ & $28.7(7)$ \\
\hline N7 & $3285.2(18)$ & $962.5(13)$ & 6090.1(11) & $14.3(5)$ \\
\hline $\mathrm{C} 28$ & $4444(2)$ & 1302.3(17) & 5982.3(14) & $20.2(6)$ \\
\hline C29 & $4574(2)$ & $1538.9(19)$ & $5261.7(15)$ & $26.2(7)$ \\
\hline C30 & $5743(3)$ & $1780(2)$ & $5190.6(18)$ & $34.6(8)$ \\
\hline C31 & $6068(3)$ & $1114(2)$ & $5080.6(19)$ & $38.1(8)$ \\
\hline C32 & $2745(2)$ & 173.2(15) & $5700.7(14)$ & $16.6(5)$ \\
\hline C33 & $1593(2)$ & $-254.0(16)$ & $5771.1(15)$ & $18.6(6)$ \\
\hline C34 & $1150(3)$ & $-1057.8(17)$ & $5408.4(16)$ & $26.2(7)$ \\
\hline C35 & $-4(3)$ & $-1488.8(19)$ & $5467.0(18)$ & $33.6(8)$ \\
\hline C36 & $2660(2)$ & $1472.4(16)$ & 5830.3(14) & $18.5(6)$ \\
\hline C37 & $3153(2)$ & $2302.2(16)$ & $6136.7(15)$ & $22.9(6)$ \\
\hline C38 & $2435(3)$ & 2753.1(17) & $5870.1(16)$ & $25.2(7)$ \\
\hline C39 & 1435(3) & 2565.6(19) & $6175.9(17)$ & $30.4(7)$ \\
\hline $\mathrm{C} 40$ & $3299(2)$ & $917.2(15)$ & $6852.5(13)$ & $16.1(5)$ \\
\hline C41 & $3715(2)$ & $327.3(16)$ & 7196.8(14) & $18.6(6)$ \\
\hline C42 & $3994(3)$ & $538.4(17)$ & 7963.0(15) & $23.8(6)$ \\
\hline C43 & $4316(3)$ & $-58.6(18)$ & $8371.9(16)$ & $28.9(7)$ \\
\hline N7A & $1692(3)$ & $4171.5(19)$ & $3939(2)$ & $16.0(14)$ \\
\hline $\mathrm{C} 21 \mathrm{~A}$ & $1926(4)$ & $4524(2)$ & $3282(2)$ & $20.0(11)$ \\
\hline $\mathrm{C} 22 \mathrm{~A}$ & 1161(5) & $4129(4)$ & $2640(3)$ & $37.0(15)$ \\
\hline $\mathrm{C} 23 \mathrm{~A}$ & $1561(5)$ & $4534(4)$ & $2038(3)$ & $38.7(15)$ \\
\hline $\mathrm{C} 24 \mathrm{~A}$ & $799(5)$ & $4255(4)$ & 1374(3) & 44.1(18) \\
\hline $\mathrm{C} 25 \mathrm{~A}$ & $2573(4)$ & $4630(2)$ & $4530(2)$ & $21.7(11)$ \\
\hline C26A & $2627(4)$ & $5447(3)$ & $4691(3)$ & $26.1(12)$ \\
\hline $\mathrm{C} 27 \mathrm{~A}$ & $3757(4)$ & $5961(3)$ & $5041(3)$ & $25.9(12)$ \\
\hline $\mathrm{C} 28 \mathrm{~A}$ & $4576(7)$ & $6163(7)$ & $4580(6)$ & $36(3)$ \\
\hline C29A & $602(4)$ & $4151(3)$ & $4049(2)$ & $27.9(11)$ \\
\hline C30A & $246(4)$ & $3833(3)$ & $4696(2)$ & $27.5(10)$ \\
\hline C31A & $-876(7)$ & $3839(5)$ & 4707(5) & $35(2)$ \\
\hline C32A & $-1314(5)$ & $3451(5)$ & $5304(3)$ & $49(2)$ \\
\hline C33A & $1648(4)$ & $3334(2)$ & $3883(2)$ & $16.4(9)$ \\
\hline C34A & $2646(5)$ & $3251(3)$ & $3710(5)$ & $20.6(10)$ \\
\hline C35A & $2656(5)$ & 2438(3) & $3818(6)$ & $20(1)$ \\
\hline C36A & $3686(9)$ & 2388(6) & $3676(11)$ & $27(3)$ \\
\hline N7B & $1780(3)$ & $4106(2)$ & $3773(3)$ & 17.4(18) \\
\hline C21B & $1349(5)$ & $4019(3)$ & $3018(3)$ & 28.5(17) \\
\hline $\mathrm{C} 22 \mathrm{~B}$ & $1258(6)$ & $4710(3)$ & $2682(3)$ & $24.2(15)$ \\
\hline C23B & $801(6)$ & $4494(3)$ & $1931(3)$ & $28.7(16)$ \\
\hline C24B & $635(6)$ & $5131(4)$ & 1539(3) & $28.8(16)$ \\
\hline C25B & $2847(4)$ & $4782(3)$ & $3947(2)$ & $20.1(14)$ \\
\hline C26B & $3401(5)$ & $4900(4)$ & $4695(3)$ & $25.7(15)$ \\
\hline C27B & $4543(4)$ & $5487(4)$ & 4794(3) & $28.0(16)$ \\
\hline C28B & $4617(9)$ & $6296(6)$ & $4628(10)$ & $46(5)$ \\
\hline C29B & $980(4)$ & $4224(3)$ & $4166(3)$ & $27.9(11)$ \\
\hline C30B & $15(4)$ & $3524(3)$ & 4239(3) & $27.5(10)$ \\
\hline C31B & $-674(9)$ & $3752(5)$ & $4665(7)$ & $23(3)$ \\
\hline C32B & $-1557(5)$ & $3059(4)$ & $4835(4)$ & $30.5(17)$ \\
\hline C33B & 1947(5) & $3355(3)$ & $3980(3)$ & $16.4(9)$ \\
\hline C34B & 2888(7) & $3243(4)$ & $3748(6)$ & $20.6(10)$ \\
\hline C35B & $2885(7)$ & $2426(4)$ & $3850(7)$ & $20(1)$ \\
\hline C36B & $3889(11)$ & $2359(6)$ & $3680(11)$ & $20(3)$ \\
\hline
\end{tabular}




\begin{tabular}{lllll}
\hline Atom & $\mathbf{x}$ & $\mathbf{y}$ & $\mathbf{z}$ & \multicolumn{1}{c}{$\boldsymbol{U}_{\text {eq }}$} \\
\hline C14_1 & $-430(9)$ & $8052(6)$ & $1095(5)$ & $15.4(7)$ \\
C15_1 & $377(11)$ & $7868(7)$ & $1496(5)$ & $19.5(9)$ \\
C16_1 & $648(6)$ & $7268(4)$ & $1273(3)$ & $19.6(15)$ \\
C17_1 & $119(5)$ & $6842(3)$ & $654(2)$ & $18.8(14)$ \\
C18_1 & $374(5)$ & $6160(3)$ & $437(3)$ & $31.1(15)$ \\
C19_1 & $-667(5)$ & $7046(3)$ & $248(2)$ & $17.7(15)$ \\
C20_1 & $-944(8)$ & $7647(5)$ & $466(4)$ & $18.4(16)$ \\
C14_2 & $-527(9)$ & $8125(6)$ & $1062(4)$ & $15.4(7)$ \\
C15_2 & $280(11)$ & $7880(8)$ & $1377(5)$ & $19.5(9)$ \\
C16_2 & $477(6)$ & $7296(4)$ & $1055(3)$ & $18.0(15)$ \\
C17_2 & $-128(5)$ & $6946(3)$ & $422(2)$ & $14.0(13)$ \\
C18_2 & $67(5)$ & $6291(3)$ & $88(3)$ & $24.7(13)$ \\
C19_2 & $-904(5)$ & $7218(3)$ & $99(3)$ & $14.2(13)$ \\
C20_2 & $-1108(8)$ & $7802(5)$ & $417(4)$ & $12.6(13)$ \\
C14_3 & $2511(7)$ & $9181(2)$ & $3228(5)$ & $16.3(9)$ \\
C15_3 & $1612(10)$ & $8645(3)$ & $3413(9)$ & $21.4(10)$ \\
C16_3 & $1380(9)$ & $7863(3)$ & $3280(9)$ & $24(2)$ \\
C17_3 & $2033(6)$ & $7604(2)$ & $2959(5)$ & $21.2(17)$ \\
C18_3 & $1811(8)$ & $6752(2)$ & $2854(6)$ & $30(2)$ \\
C19_3 & $2931(5)$ & $8147(3)$ & $2774(4)$ & $22.3(10)$ \\
C20_3 & $3167(7)$ & $8931(3)$ & $2903(6)$ & $20.1(16)$ \\
C14_4 & $2396(7)$ & $9008(2)$ & $3269(5)$ & $16.3(9)$ \\
C15_4 & $1462(9)$ & $8509(3)$ & $3446(10)$ & $21.4(10)$ \\
C16_4 & $1188(9)$ & $7718(3)$ & $3340(9)$ & $19.9(17)$ \\
C17_4 & $1836(6)$ & $7414(2)$ & $3059(5)$ & $19.3(16)$ \\
C18_4 & $1548(7)$ & $6553(2)$ & $2965(6)$ & $27.2(19)$ \\
C19_4 & $2725(5)$ & $7921(3)$ & $2837(4)$ & $22.3(10)$ \\
C20_4 & $3019(7)$ & $8714(3)$ & $2953(6)$ & $20.8(16)$ \\
C14_5 & $4477.7(19)$ & $3146.9(13)$ & $1138.6(11)$ & $15.4(5)$ \\
C15_5 & $5295(2)$ & $2918.8(14)$ & $1464.2(12)$ & $22.4(6)$ \\
C16_5 & $5483(2)$ & $2315.4(15)$ & $1168.1(14)$ & $25.4(7)$ \\
C17_5 & $4870(2)$ & $1936.2(13)$ & $545.5(13)$ & $20.5(6)$ \\
C18_5 & $5056(3)$ & $1262.9(15)$ & $238.5(16)$ & $29.4(7)$ \\
C19_5 & $4067(2)$ & $2181.5(13)$ & $217.3(11)$ & $18.3(6)$ \\
C20_5 & $3870.8(19)$ & $2783.0(14)$ & $511.0(11)$ & $15.8(5)$ \\
C14_6 & $7611(2)$ & $4242.7(12)$ & $3163.1(15)$ & $27.6(7)$ \\
C15_6 & $6723(2)$ & $3742.4(14)$ & $3386.5(17)$ & $35.5(8)$ \\
C16_6 & $6462(2)$ & $2951.5(14)$ & $3292.5(18)$ & $40.9(9)$ \\
C17_6 & $7068(2)$ & $2649.5(13)$ & $2965.8(17)$ & $38.8(9)$ \\
C18_6 & $6812(3)$ & $1790.1(14)$ & $2898(2)$ & $52.0(12)$ \\
C19_6 & $7923(2)$ & $3156.6(14)$ & $2712.5(16)$ & $37.0(8)$ \\
C20_6 & $8197(2)$ & $3949.3(14)$ & $2811.0(16)$ & $32.5(8)$ \\
& & & &
\end{tabular}

Table 2: Anisotropic Displacement Parameters $\left(\times 10^{4}\right)$ CMW04_BuTsNH2OH. The anisotropic displacement factor exponent takes the form: $-2 \pi^{2}\left[h^{2} a^{* 2} \times U_{11}+\ldots+2 h k a^{*} \times b^{*} \times U_{12}\right]$

\begin{tabular}{llllccc}
\hline Atom & \multicolumn{1}{c}{$\boldsymbol{U}_{\mathbf{1 1}}$} & \multicolumn{1}{c}{$\boldsymbol{U}_{\mathbf{2 2}}$} & \multicolumn{1}{c}{$\boldsymbol{U}_{\mathbf{3 3}}$} & $\boldsymbol{U}_{\mathbf{2 3}}$ & $\boldsymbol{U}_{\mathbf{1 3}}$ & $\boldsymbol{U}_{\mathbf{1 2}}$ \\
\hline Zn1 & $15.27(15)$ & $11.21(15)$ & $10.30(14)$ & $0.18(11)$ & $2.42(11)$ & $2.73(12)$ \\
S1 & $11.8(3)$ & $16.9(3)$ & $17.7(3)$ & $-5.7(2)$ & $3.2(2)$ & $3.6(2)$ \\
S2 & $17.8(3)$ & $11.7(3)$ & $16.2(3)$ & $-1.7(2)$ & $5.4(3)$ & $0.1(2)$ \\
S3 & $25.8(4)$ & $15.6(3)$ & $23.9(4)$ & $3.3(3)$ & $-9.1(3)$ & $5.5(3)$ \\
01 & $17.2(10)$ & $26.9(11)$ & $22.8(11)$ & $-3.9(9)$ & $9.7(8)$ & $1.1(9)$ \\
O2 & $17.4(10)$ & $21(1)$ & $28.9(11)$ & $-11.9(9)$ & $1.7(8)$ & $7.2(8)$ \\
03 & $16.5(10)$ & $19.7(10)$ & $33.1(12)$ & $-2.8(9)$ & $5.2(9)$ & $3.2(8)$ \\
04 & $36.5(13)$ & $15.7(10)$ & $19.7(11)$ & $-0.6(8)$ & $13.3(9)$ & $-3.2(9)$ \\
05 & $40.0(13)$ & $21.2(11)$ & $18.2(10)$ & $4.5(8)$ & $-4.9(9)$ & $5.7(10)$ \\
06 & $26.3(12)$ & $24.3(12)$ & $43.3(14)$ & $5.3(10)$ & $-15.8(10)$ & $5.7(10)$
\end{tabular}




\begin{tabular}{|c|c|c|c|c|c|c|}
\hline Atom & $U_{11}$ & $U_{22}$ & $\boldsymbol{U}_{33}$ & $U_{23}$ & $U_{13}$ & $U_{12}$ \\
\hline 07 & $56.2(16)$ & $18.2(11)$ & $17.1(11)$ & $3.8(9)$ & $11.5(11)$ & $-6.9(11)$ \\
\hline $\mathrm{N} 2$ & $19.9(12)$ & $17.8(12)$ & $17.8(12)$ & $2.8(9)$ & $0.5(9)$ & $9.1(10)$ \\
\hline $\mathrm{N} 1$ & $11.4(10)$ & $11.7(10)$ & $13.6(10)$ & $-0.1(8)$ & $4.4(8)$ & $2.2(8)$ \\
\hline N3 & $20.8(12)$ & $10.6(10)$ & $11.6(11)$ & $-0.6(8)$ & $3.7(9)$ & $-0.7(9)$ \\
\hline N5 & $32.7(14)$ & $16.7(12)$ & $14.3(11)$ & $3.8(9)$ & $7.4(10)$ & $3.1(10)$ \\
\hline $\mathrm{N} 4$ & $13.2(11)$ & $11.7(10)$ & $14.9(11)$ & $-0.4(8)$ & $5.1(9)$ & $2.8(8)$ \\
\hline C7 & $8.2(12)$ & $21.9(14)$ & $22.8(14)$ & $-5.6(11)$ & $-0.8(10)$ & $5.6(11)$ \\
\hline C8 & $14.1(13)$ & $20.3(14)$ & $25.0(15)$ & $-5.1(11)$ & 1.1(11) & $8.5(11)$ \\
\hline C12 & $15.3(14)$ & $18.4(14)$ & $32.3(17)$ & $-6.4(12)$ & $-3.5(12)$ & $6.9(11)$ \\
\hline C1 & $14.6(13)$ & $14.4(12)$ & $13.7(12)$ & $3.4(10)$ & $3.9(10)$ & $3(1)$ \\
\hline $\mathrm{C} 2$ & $15.1(13)$ & $11.1(12)$ & $15.7(12)$ & $1.6(10)$ & $3.6(10)$ & $4.7(10)$ \\
\hline C3 & $19.2(13)$ & $15.1(13)$ & $12.5(12)$ & $0.6(10)$ & $6.1(10)$ & $5.6(11)$ \\
\hline C10 & $13.9(13)$ & $29.6(16)$ & $27.7(16)$ & $-6.1(13)$ & $-1.0(12)$ & $11.4(12)$ \\
\hline C4 & $19.3(13)$ & $14.9(13)$ & $10.0(12)$ & $-1.1(10)$ & $2.7(10)$ & $2.8(10)$ \\
\hline C11 & $18.7(15)$ & $24.9(15)$ & $31.9(17)$ & $-1.0(13)$ & $-3.2(12)$ & $10.8(12)$ \\
\hline C6 & $19.4(14)$ & $22.5(15)$ & $24.1(15)$ & $-0.3(12)$ & $2.9(12)$ & $9.6(12)$ \\
\hline C13 & $25.8(16)$ & $34.0(18)$ & $26.7(16)$ & $-7.2(13)$ & $-5.0(13)$ & $17.3(14)$ \\
\hline C5 & $11.5(12)$ & $18.7(13)$ & $20.9(14)$ & $-1.1(11)$ & $3.8(10)$ & $3.7(10)$ \\
\hline C9 & $15.9(14)$ & $20.1(14)$ & $29.8(16)$ & $-8.7(12)$ & $0.7(12)$ & $7.3(11)$ \\
\hline Zn1B & $14.46(16)$ & $19.78(17)$ & $8.99(15)$ & $1.07(12)$ & $1.61(11)$ & $2.24(12)$ \\
\hline S1B & $20.1(3)$ & $16.5(3)$ & 15.1(3) & $1.7(3)$ & $2.7(3)$ & $4.3(3)$ \\
\hline S2B & $15.1(3)$ & $16.5(3)$ & $18.5(3)$ & $2.3(3)$ & $1.7(3)$ & $0.6(3)$ \\
\hline S3BA & $19.7(7)$ & $16.9(13)$ & $12.6(4)$ & $-1.1(13)$ & $-4.3(4)$ & $5.1(15)$ \\
\hline S3BB & $19.7(7)$ & $16.9(13)$ & $12.6(4)$ & $-1.1(13)$ & $-4.3(4)$ & $5.1(15)$ \\
\hline 01B & $23.6(11)$ & $24.9(11)$ & $20.4(10)$ & $7.5(8)$ & $9.8(8)$ & $7.7(9)$ \\
\hline O2B & $30.3(12)$ & $22.1(11)$ & $23.4(11)$ & $-4.0(9)$ & $-1.2(9)$ & $8.7(9)$ \\
\hline O3B & $18.7(11)$ & $25.9(12)$ & $46.4(15)$ & $6.3(10)$ & $-8.5(10)$ & $1.6(9)$ \\
\hline O4B & $31.1(12)$ & $18.1(10)$ & $26.3(11)$ & $4.1(9)$ & $15.1(9)$ & $3.1(9)$ \\
\hline 05BA & $23(4)$ & $23(4)$ & 9.1(15) & $1(3)$ & $2(2)$ & $1(3)$ \\
\hline 05BB & $23(4)$ & $23(4)$ & $9.1(15)$ & $1(3)$ & $2(2)$ & $1(3)$ \\
\hline 06BA & $22.2(13)$ & $24(4)$ & $23.6(13)$ & $2(3)$ & $-9(1)$ & $7(3)$ \\
\hline O6BB & $22.2(13)$ & $24(4)$ & $23.6(13)$ & $2(3)$ & $-9(1)$ & $7(3)$ \\
\hline O7B & $34.7(12)$ & $19.2(10)$ & $22.8(11)$ & $7.4(8)$ & $15.6(10)$ & $6.6(9)$ \\
\hline $\mathrm{N} 1 \mathrm{~B}$ & $16.5(11)$ & $21.6(11)$ & $10.9(10)$ & $-2.1(8)$ & 2.8(8) & $-2.9(9)$ \\
\hline $\mathrm{N} 2 \mathrm{BA}$ & $15.7(11)$ & $17(2)$ & $14.1(10)$ & $0.6(13)$ & $1.3(8)$ & $6.9(15)$ \\
\hline $\mathrm{N} 2 \mathrm{BB}$ & $15.7(11)$ & $17(2)$ & $14.1(10)$ & $0.6(13)$ & $1.3(8)$ & $6.9(15)$ \\
\hline N3B & $17.6(11)$ & $17.6(11)$ & $15.2(11)$ & $2.3(9)$ & $2.7(9)$ & $2.1(9)$ \\
\hline N4B & $17.9(11)$ & $15.3(11)$ & $11.5(10)$ & $1.5(8)$ & $1.0(9)$ & $-0.5(9)$ \\
\hline N5B & $23.0(13)$ & $24.7(13)$ & $14.3(11)$ & $6.4(10)$ & $8.1(10)$ & $8(1)$ \\
\hline $\mathrm{C} 2 \mathrm{~B}$ & $15.1(13)$ & $46.2(18)$ & $17.4(13)$ & $-1.6(12)$ & $3.7(11)$ & $6.2(13)$ \\
\hline C3B & $23.4(14)$ & $20.5(13)$ & $13.8(12)$ & $1.9(10)$ & $4.2(11)$ & $-2.9(11)$ \\
\hline C4B & $22.5(14)$ & $17.6(13)$ & $18.0(13)$ & $1.3(10)$ & $4.7(11)$ & $-1.8(11)$ \\
\hline C5B & $26.9(15)$ & $22.7(14)$ & $10.9(12)$ & $0.2(10)$ & $5.3(10)$ & $-0.2(11)$ \\
\hline C1B & $14.8(13)$ & $36.2(16)$ & $17.6(13)$ & $-4.7(12)$ & $5.4(10)$ & $-0.8(11)$ \\
\hline C6B & $23.9(14)$ & $19.5(13)$ & $10.4(12)$ & $0.7(10)$ & $-0.2(10)$ & $-0.5(11)$ \\
\hline C7B & $17.5(14)$ & $22.5(14)$ & $15.4(13)$ & $4.5(11)$ & $2.3(11)$ & $3.7(11)$ \\
\hline C8B & $24.0(15)$ & $17.8(14)$ & $24.5(15)$ & $5.0(12)$ & $4.2(12)$ & $2.8(12)$ \\
\hline C9B & $21.4(15)$ & $22.7(15)$ & $23.6(15)$ & $-4.8(12)$ & $1.5(12)$ & $2.9(12)$ \\
\hline C10B & $13.6(13)$ & $31.0(16)$ & $19.5(14)$ & $2.9(12)$ & $2.6(11)$ & $7.0(12)$ \\
\hline C11B & $18.5(14)$ & $22.8(14)$ & $23.2(15)$ & $8.0(12)$ & $5.3(11)$ & $9.6(12)$ \\
\hline C12B & $17.5(14)$ & $16.6(14)$ & $24.7(15)$ & $2.4(11)$ & $1.0(11)$ & $3.2(11)$ \\
\hline C13B & $25.2(16)$ & $40.0(19)$ & $19.8(15)$ & $-0.2(13)$ & $-0.4(12)$ & $13.0(14)$ \\
\hline N7 & $13.9(11)$ & $14.4(11)$ & $9.8(10)$ & $-2.6(8)$ & $-0.5(8)$ & $0.7(9)$ \\
\hline C28 & $15.3(13)$ & $22.7(14)$ & $16.3(13)$ & $-2.1(11)$ & $4.0(11)$ & $-0.8(11)$ \\
\hline C29 & $22.0(15)$ & $29.0(16)$ & $19.2(15)$ & $-0.5(12)$ & $7.9(12)$ & $-2.5(13)$ \\
\hline C30 & $24.8(17)$ & $39(2)$ & $31.6(18)$ & $-2.1(15)$ & $11.4(14)$ & $-1.3(14)$ \\
\hline C31 & $27.9(18)$ & $48(2)$ & $35.6(19)$ & $4.9(16)$ & $2.8(15)$ & $11.7(16)$ \\
\hline C32 & $18.3(13)$ & $13.9(13)$ & $15.3(13)$ & $-4(1)$ & $2.6(10)$ & $3.6(11)$ \\
\hline
\end{tabular}




\begin{tabular}{|c|c|c|c|c|c|c|}
\hline Atom & $U_{11}$ & $U_{22}$ & $U_{33}$ & $U_{23}$ & $U_{13}$ & $U_{12}$ \\
\hline C33 & $17.5(14)$ & $14.5(13)$ & $19.1(14)$ & $-2(1)$ & $3.6(11)$ & $0.4(11)$ \\
\hline C34 & $28.2(16)$ & $16.3(14)$ & $26.8(16)$ & $-5.8(12)$ & $3.1(13)$ & $0.7(12)$ \\
\hline C35 & $36.0(19)$ & $20.7(16)$ & 28.7(17) & $-3.4(13)$ & $0.1(14)$ & $-4.6(14)$ \\
\hline C36 & $20.9(14)$ & $14.8(13)$ & $17.3(13)$ & $3(1)$ & $5.5(11)$ & $2.7(11)$ \\
\hline C37 & $25.6(15)$ & $13.9(13)$ & 21.3(14) & $-1.6(11)$ & $-0.5(12)$ & $0.4(11)$ \\
\hline C38 & 31.1(17) & $15.0(14)$ & $24.0(15)$ & $1.6(11)$ & $-1.0(13)$ & $4.8(12)$ \\
\hline C39 & $42(2)$ & $24.3(16)$ & $25.5(16)$ & $-3.0(13)$ & $1.3(14)$ & $15.1(15)$ \\
\hline C40 & $20.1(14)$ & $12.7(12)$ & $12.4(12)$ & $-1.6(10)$ & $3.9(10)$ & $2(1)$ \\
\hline C41 & $19.7(14)$ & $15.6(13)$ & $18.2(14)$ & $1.4(10)$ & $2.6(11)$ & $4.1(11)$ \\
\hline C42 & $31.0(17)$ & $20.3(14)$ & $16.8(14)$ & $1.7(11)$ & $1.2(12)$ & $7.3(13)$ \\
\hline $\mathrm{C} 43$ & $34.0(18)$ & $24.9(16)$ & $21.8(15)$ & $1.9(12)$ & $-3.2(13)$ & $7.6(14)$ \\
\hline N7A & 18(3) & $14(2)$ & $13(2)$ & $5.2(17)$ & $-0.8(18)$ & $3.8(19)$ \\
\hline $\mathrm{C} 21 \mathrm{~A}$ & $16(2)$ & $17(2)$ & $26(2)$ & $10.7(19)$ & $3.0(18)$ & $5.2(18)$ \\
\hline $\mathrm{C} 22 \mathrm{~A}$ & $36(2)$ & $38(2)$ & $32(2)$ & $8.0(17)$ & $5.0(17)$ & $8.6(16)$ \\
\hline C23A & $40(2)$ & $39(2)$ & $35(2)$ & $7.4(16)$ & $10.0(17)$ & $9.6(16)$ \\
\hline $\mathrm{C} 24 \mathrm{~A}$ & $43(4)$ & $50(4)$ & $24(3)$ & $6(3)$ & $2(3)$ & $1(3)$ \\
\hline C25A & 22.1(18) & 17.3(17) & 21.6(18) & $-0.2(14)$ & $0.1(14)$ & $4.5(14)$ \\
\hline $\mathrm{C} 26 \mathrm{~A}$ & $29(3)$ & $17(3)$ & $31(3)$ & $2(2)$ & $3(2)$ & $8(2)$ \\
\hline C27A & $31(3)$ & $14(2)$ & $23(3)$ & $-3(2)$ & $3(2)$ & $-2(2)$ \\
\hline $\mathrm{C} 28 \mathrm{~A}$ & $42(6)$ & $28(4)$ & $18(5)$ & $-7(4)$ & $14(4)$ & $-17(3)$ \\
\hline C29A & $27(2)$ & $26.3(15)$ & $29.9(16)$ & $7.9(12)$ & $6.7(15)$ & $8.6(15)$ \\
\hline C30A & $26(2)$ & $25(2)$ & $24.3(19)$ & $4.6(16)$ & $-0.2(17)$ & $3.9(18)$ \\
\hline C31A & $26(4)$ & $49(5)$ & $28(4)$ & $16(4)$ & $2(3)$ & $14(4)$ \\
\hline $\mathrm{C} 32 \mathrm{~A}$ & $27(3)$ & $72(5)$ & $22(3)$ & $17(3)$ & $-2(3)$ & $-9(3)$ \\
\hline C33A & $15.4(19)$ & $13.6(12)$ & $13.9(14)$ & $3.6(10)$ & $-5.5(14)$ & $1.7(12)$ \\
\hline C34A & $22(2)$ & $16.6(12)$ & $19.9(14)$ & $1.4(10)$ & 2.3(18) & $4.2(12)$ \\
\hline C35A & $22(2)$ & $18.6(12)$ & $18.6(14)$ & $3.7(10)$ & 2.1(18) & $6.9(12)$ \\
\hline C36A & $28(4)$ & $24(4)$ & $24(4)$ & $0(3)$ & $2(3)$ & $5(3)$ \\
\hline N7B & $18(4)$ & $13(3)$ & $18(3)$ & $-4(2)$ & $4(3)$ & $2(3)$ \\
\hline C21B & $34(4)$ & $17(3)$ & $24(4)$ & $-7(3)$ & $-3(3)$ & $2(3)$ \\
\hline C22B & $24(3)$ & $20(3)$ & $26(3)$ & $-3(2)$ & $2(3)$ & $7(3)$ \\
\hline C23B & $26(4)$ & $34(4)$ & $25(3)$ & $-3(3)$ & $10(3)$ & $8(3)$ \\
\hline C24B & $17(3)$ & $30(4)$ & $32(4)$ & $-8(3)$ & $5(3)$ & $1(3)$ \\
\hline C25B & $19(2)$ & $19(2)$ & $21(2)$ & $0.5(15)$ & $4.0(15)$ & $5.0(15)$ \\
\hline C26B & $31(4)$ & $17(3)$ & $22(3)$ & $4(2)$ & $3(3)$ & $1(3)$ \\
\hline C27B & $30(4)$ & $29(4)$ & $17(3)$ & $-1(3)$ & $3(3)$ & $2(3)$ \\
\hline C28B & $65(10)$ & $22(5)$ & $33(8)$ & $2(4)$ & $-11(6)$ & $5(5)$ \\
\hline C29B & $27(2)$ & $26.3(15)$ & $29.9(16)$ & $7.9(12)$ & $6.7(15)$ & $8.6(15)$ \\
\hline C30B & $26(2)$ & $25(2)$ & $24.3(19)$ & $4.6(16)$ & $-0.2(17)$ & $3.9(18)$ \\
\hline C31B & $23(3)$ & $23(3)$ & $22(3)$ & $3.9(18)$ & $4.3(19)$ & $7.4(19)$ \\
\hline C32B & $30(4)$ & $32(4)$ & $28(4)$ & $7(3)$ & $9(3)$ & $8(3)$ \\
\hline C33B & $15.4(19)$ & $13.6(12)$ & $13.9(14)$ & $3.6(10)$ & $-5.5(14)$ & $1.7(12)$ \\
\hline C34B & $22(2)$ & $16.6(12)$ & $19.9(14)$ & $1.4(10)$ & 2.3(18) & $4.2(12)$ \\
\hline C35B & $22(2)$ & $18.6(12)$ & $18.6(14)$ & $3.7(10)$ & 2.1(18) & $6.9(12)$ \\
\hline C36B & $18(5)$ & $24(5)$ & $11(4)$ & $-2(3)$ & $2(5)$ & $-2(3)$ \\
\hline C14_1 & $14.9(14)$ & 13.7(15) & $14.8(12)$ & 1.5(11) & $3.9(10)$ & $1.5(9)$ \\
\hline C15_1 & $17(2)$ & $17.1(13)$ & $18(3)$ & $1(2)$ & $-0.5(17)$ & $-0.2(12)$ \\
\hline C16_1 & $16(3)$ & $22(3)$ & $18(4)$ & $5(3)$ & $1(3)$ & $4(2)$ \\
\hline C17_1 & $11(3)$ & $25(3)$ & $20(3)$ & $4(3)$ & $6(3)$ & $5(2)$ \\
\hline C18_1 & $30(4)$ & $26(3)$ & $36(4)$ & $-4(3)$ & $8(3)$ & $9(3)$ \\
\hline C19_1 & $23(4)$ & $23(4)$ & $8(3)$ & $4(3)$ & $4(3)$ & $9(3)$ \\
\hline C20_1 & $12(4)$ & $22(5)$ & $16(3)$ & $1(3)$ & $-2(2)$ & $3(3)$ \\
\hline C14_2 & $14.9(14)$ & 13.7(15) & $14.8(12)$ & $1.5(11)$ & $3.9(10)$ & $1.5(9)$ \\
\hline C15_2 & $17(2)$ & 17.1(13) & $18(3)$ & $1(2)$ & $-0.5(17)$ & $-0.2(12)$ \\
\hline C16_2 & $15(3)$ & $20(3)$ & $16(3)$ & $3(2)$ & $-2(2)$ & $4(2)$ \\
\hline C17_2 & $12(3)$ & $15(3)$ & $14(3)$ & $4(2)$ & $1(2)$ & $4(2)$ \\
\hline C18_2 & $23(3)$ & $23(3)$ & $30(4)$ & $-3(3)$ & $6(3)$ & $10(3)$ \\
\hline C19_2 & $14(3)$ & $14(3)$ & $12(3)$ & $1(2)$ & $-1(2)$ & $5(2)$ \\
\hline
\end{tabular}




\begin{tabular}{lllllcc}
\hline Atom & \multicolumn{1}{c}{$\boldsymbol{U}_{\mathbf{1 1}}$} & \multicolumn{1}{c}{$\boldsymbol{U}_{\mathbf{2 2}}$} & \multicolumn{1}{c}{$\boldsymbol{U}_{\mathbf{3 3}}$} & \multicolumn{1}{c}{$\boldsymbol{U}_{\mathbf{2 3}}$} & $\boldsymbol{U}_{\mathbf{1 3}}$ & $\boldsymbol{U}_{\mathbf{1 2}}$ \\
\hline C20_2 & $10(3)$ & $13(3)$ & $14(2)$ & $1(2)$ & $2.9(19)$ & $3(2)$ \\
C14_3 & $17.3(14)$ & $17.0(17)$ & $13.8(13)$ & $0.8(16)$ & $-2(1)$ & $7.5(14)$ \\
C15_3 & $20(3)$ & $23(2)$ & $22.5(19)$ & $0(3)$ & $4.3(19)$ & $10(2)$ \\
C16_3 & $19(5)$ & $25(4)$ & $20(4)$ & $0(4)$ & $2(4)$ & $1(3)$ \\
C17_3 & $25(4)$ & $24(4)$ & $16(4)$ & $2(3)$ & $1(3)$ & $13(3)$ \\
C18_3 & $27(5)$ & $27(4)$ & $38(5)$ & $4(4)$ & $13(4)$ & $7(4)$ \\
C19_3 & $19.9(16)$ & $22.9(19)$ & $25.8(15)$ & $2.5(14)$ & $3.5(12)$ & $10.4(15)$ \\
C20_3 & $15(4)$ & $20(3)$ & $25(4)$ & $6(4)$ & $-3(3)$ & $9(3)$ \\
C14_4 & $17.3(14)$ & $17.0(17)$ & $13.8(13)$ & $0.8(16)$ & $-2(1)$ & $7.5(14)$ \\
C15_4 & $20(3)$ & $23(2)$ & $22.5(19)$ & $0(3)$ & $4.3(19)$ & $10(2)$ \\
C16_4 & $16(4)$ & $16(3)$ & $27(4)$ & $0(3)$ & $2(3)$ & $6(3)$ \\
C17_4 & $17(3)$ & $20(3)$ & $21(4)$ & $2(2)$ & $-2(2)$ & $10(2)$ \\
C18_4 & $24(5)$ & $23(3)$ & $38(5)$ & $2(3)$ & $6(3)$ & $13(3)$ \\
C19_4 & $19.9(16)$ & $22.9(19)$ & $25.8(15)$ & $2.5(14)$ & $3.5(12)$ & $10.4(15)$ \\
C20_4 & $16(3)$ & $25(3)$ & $23(4)$ & $3(3)$ & $3(2)$ & $9(2)$ \\
C14_5 & $14.6(13)$ & $11.0(12)$ & $15.4(13)$ & $1.6(10)$ & $2.8(10)$ & $-1.3(10)$ \\
C15_5 & $23.0(15)$ & $18.3(14)$ & $18.1(14)$ & $-2.4(11)$ & $-3.9(11)$ & $2.1(12)$ \\
C16_5 & $20.0(15)$ & $20.1(15)$ & $31.7(17)$ & $-0.6(12)$ & $-4.7(12)$ & $6.8(12)$ \\
C17_5 & $19.3(14)$ & $11.6(13)$ & $28.1(15)$ & $1.1(11)$ & $6.3(12)$ & $2.0(11)$ \\
C18_5 & $29.4(17)$ & $19.5(15)$ & $38.8(19)$ & $-3.1(13)$ & $7.1(14)$ & $8.3(13)$ \\
C19_5 & $19.3(14)$ & $15.1(13)$ & $14.9(13)$ & $-0.7(10)$ & $1.6(11)$ & $0.5(11)$ \\
C20_5 & $13.1(12)$ & $16.1(13)$ & $15.1(13)$ & $3.1(10)$ & $1.2(10)$ & $2.4(10)$ \\
C14_6 & $25.4(16)$ & $18.9(15)$ & $32.4(17)$ & $5.1(12)$ & $-10.5(13)$ & $8.4(12)$ \\
C15_6 & $33.1(19)$ & $21.6(16)$ & $47(2)$ & $9.0(14)$ & $-5.1(16)$ & $10.6(14)$ \\
C16_6 & $33.8(19)$ & $18.3(16)$ & $63(2)$ & $9.2(15)$ & $-7.3(17)$ & $8.2(14)$ \\
C17_6 & $34.0(18)$ & $18.6(15)$ & $55(2)$ & $1.0(14)$ & $-18.7(16)$ & $12.5(13)$ \\
C18_6 & $52(3)$ & $18.5(17)$ & $75(3)$ & $-3.0(18)$ & $-19(2)$ & $14.6(17)$ \\
C19_6 & $36.0(19)$ & $28.7(17)$ & $43(2)$ & $-3.2(14)$ & $-16.1(15)$ & $19.3(15)$ \\
C20_6 & $29.8(17)$ & $24.7(16)$ & $36.4(19)$ & $4.5(13)$ & $-11.0(14)$ & $10.5(13)$
\end{tabular}

Table 3: Bond Lengths in Å for CMW04_BuTsNH2OH.

\begin{tabular}{lll}
\hline Atom & Atom & Length/Å \\
\hline Zn1 & N2 & $2.078(2)$ \\
Zn1 & N1 & $2.247(2)$ \\
Zn1 & N3 & $2.044(2)$ \\
Zn1 & N5 & $2.111(2)$ \\
Zn1 & N4 & $2.106(2)$ \\
S1 & O1 & $1.449(2)$ \\
S1 & O2 & $1.452(2)$ \\
S1 & N4 & $1.570(2)$ \\
S1 & C7 & $1.778(3)$ \\
S2 & O3 & $1.443(2)$ \\
S2 & O4 & $1.455(2)$ \\
S2 & N3 & $1.576(2)$ \\
S2 & C14_5 & $1.777(2)$ \\
S3 & O5 & $1.448(3)$ \\
S3 & O6 & $1.449(2)$ \\
S3 & N2 & $1.571(2)$ \\
S3 & C14_6 & $1.786(2)$ \\
07 & N5 & $1.440(3)$ \\
N2 & C6 & $1.476(4)$ \\
N1 & C1 & $1.473(3)$ \\
N1 & C3 & $1.474(3)$ \\
N1 & C5 & $1.474(3)$ \\
N3 & C4 & $1.473(3)$ \\
N4 & C2 & $1.478(3)$ \\
& &
\end{tabular}

\begin{tabular}{lll}
\hline Atom & Atom & Length/Å \\
\hline C7 & C8 & $1.393(4)$ \\
C7 & C12 & $1.394(4)$ \\
C8 & C9 & $1.379(4)$ \\
C12 & C11 & $1.390(4)$ \\
C1 & C2 & $1.523(4)$ \\
C3 & C4 & $1.515(4)$ \\
C10 & C11 & $1.390(4)$ \\
C10 & C13 & $1.509(4)$ \\
C10 & C9 & $1.393(4)$ \\
C6 & C5 & $1.513(4)$ \\
Zn1B & N1B & $2.288(2)$ \\
Zn1B & N2BA & $2.011(11)$ \\
Zn1B & N2BB & $2.119(11)$ \\
Zn1B & N3B & $2.095(2)$ \\
Zn1B & N4B & $2.036(2)$ \\
Zn1B & N5B & $2.130(2)$ \\
S1B & O1B & $1.447(2)$ \\
S1B & O2B & $1.446(2)$ \\
S1B & N3B & $1.566(3)$ \\
S1B & C7B & $1.778(3)$ \\
S2B & O3B & $1.440(2)$ \\
S2B & O4B & $1.448(2)$ \\
S2B & N4B & $1.567(2)$ \\
S2B & C14_1 & $1.861(4)$ \\
& &
\end{tabular}




\begin{tabular}{|c|c|c|}
\hline Atom & Atom & Length/Å \\
\hline S2B & C14_2 & $1.696(4)$ \\
\hline S3BA & 05BA & $1.38(2)$ \\
\hline S3BA & 06BA & $1.431(17)$ \\
\hline S3BA & N2BA & $1.576(3)$ \\
\hline S3BA & C14_3 & $1.784(3)$ \\
\hline S3BB & 05BB & $1.53(2)$ \\
\hline S3BB & O6BB & $1.459(17)$ \\
\hline S3BB & N2BB & $1.577(3)$ \\
\hline S3BB & C14_4 & $1.785(3)$ \\
\hline 07B & N5B & $1.440(3)$ \\
\hline N1B & $\mathrm{C} 3 \mathrm{~B}$ & $1.478(4)$ \\
\hline N1B & C5B & $1.467(4)$ \\
\hline N1B & C1B & $1.474(4)$ \\
\hline N2BA & $\mathrm{C} 2 \mathrm{~B}$ & $1.499(13)$ \\
\hline N2BB & $\mathrm{C} 2 \mathrm{~B}$ & $1.475(13)$ \\
\hline N3B & $\mathrm{C} 4 \mathrm{~B}$ & $1.484(4)$ \\
\hline N4B & C6B & $1.479(3)$ \\
\hline $\mathrm{C} 2 \mathrm{~B}$ & C1B & $1.512(5)$ \\
\hline C3B & $\mathrm{C} 4 \mathrm{~B}$ & $1.525(4)$ \\
\hline C5B & C6B & $1.519(4)$ \\
\hline C7B & C8B & $1.398(4)$ \\
\hline C7B & C12B & $1.386(4)$ \\
\hline C8B & С9B & $1.381(4)$ \\
\hline C9B & C10B & $1.396(4)$ \\
\hline C10B & C11B & $1.391(4)$ \\
\hline C10B & C13B & $1.514(4)$ \\
\hline C11B & $\mathrm{C} 12 \mathrm{~B}$ & $1.388(4)$ \\
\hline N7 & C28 & $1.527(4)$ \\
\hline N7 & C32 & $1.519(3)$ \\
\hline N7 & C36 & $1.523(4)$ \\
\hline N7 & $\mathrm{C} 40$ & $1.526(3)$ \\
\hline C28 & $\mathrm{C} 29$ & $1.534(4)$ \\
\hline C29 & C30 & $1.522(4)$ \\
\hline C30 & C31 & $1.483(5)$ \\
\hline C32 & C33 & $1.514(4)$ \\
\hline C33 & C34 & $1.519(4)$ \\
\hline C34 & C35 & $1.510(5)$ \\
\hline C36 & C37 & $1.518(4)$ \\
\hline C37 & C38 & $1.532(4)$ \\
\hline C38 & C39 & $1.517(5)$ \\
\hline $\mathrm{C} 40$ & C41 & $1.523(4)$ \\
\hline C41 & C42 & $1.524(4)$ \\
\hline C42 & C43 & $1.527(4)$ \\
\hline N7A & $\mathrm{C} 21 \mathrm{~A}$ & $1.509(6)$ \\
\hline N7A & $\mathrm{C} 25 \mathrm{~A}$ & $1.518(5)$ \\
\hline N7A & C29A & $1.522(6)$ \\
\hline N7A & C33A & $1.541(4)$ \\
\hline C21A & $\mathrm{C} 22 \mathrm{~A}$ & $1.493(6)$ \\
\hline C22A & $\mathrm{C} 23 \mathrm{~A}$ & $1.508(6)$ \\
\hline C23A & $\mathrm{C} 24 \mathrm{~A}$ & $1.488(6)$ \\
\hline $\mathrm{C} 25 \mathrm{~A}$ & $\mathrm{C} 26 \mathrm{~A}$ & $1.526(5)$ \\
\hline C26A & $\mathrm{C} 27 \mathrm{~A}$ & $1.522(6)$ \\
\hline C27A & $\mathrm{C} 28 \mathrm{~A}$ & $1.518(8)$ \\
\hline C29A & $\mathrm{C} 30 \mathrm{~A}$ & $1.515(3)$ \\
\hline C30A & C31A & $1.531(8)$ \\
\hline C31A & $\mathrm{C} 32 \mathrm{~A}$ & $1.506(7)$ \\
\hline C33A & C34A & $1.516(5)$ \\
\hline C34A & C35A & $1.535(4)$ \\
\hline
\end{tabular}

\begin{tabular}{|c|c|c|}
\hline Atom & Atom & Length/Å \\
\hline$\overline{C 35 A}$ & C36A & $1.510(7)$ \\
\hline N7B & C21B & $1.504(6)$ \\
\hline N7B & C25B & $1.520(5)$ \\
\hline N7B & C29B & $1.522(6)$ \\
\hline N7B & C33B & $1.543(4)$ \\
\hline $\mathrm{C} 21 \mathrm{~B}$ & C22B & $1.491(6)$ \\
\hline $\mathrm{C} 22 \mathrm{~B}$ & C23B & $1.510(6)$ \\
\hline C23B & C24B & $1.488(6)$ \\
\hline C25B & C26B & $1.527(5)$ \\
\hline C26B & C27B & $1.520(6)$ \\
\hline C27B & C28B & $1.518(8)$ \\
\hline C29B & C30B & $1.514(3)$ \\
\hline C30B & C31B & $1.531(8)$ \\
\hline C31B & C32B & $1.506(7)$ \\
\hline C33B & C34B & $1.516(5)$ \\
\hline C34B & C35B & $1.534(4)$ \\
\hline C35B & C36B & $1.510(7)$ \\
\hline C14_1 & C15_1 & $1.386(3)$ \\
\hline C14_1 & C20_1 & $1.390(3)$ \\
\hline C15_1 & C16_1 & $1.387(3)$ \\
\hline C16_1 & C17_1 & $1.392(3)$ \\
\hline C17_1 & C18_1 & $1.510(3)$ \\
\hline C17_1 & C19_1 & $1.390(3)$ \\
\hline C19_1 & C20_1 & $1.389(3)$ \\
\hline C14_2 & C15_2 & $1.386(3)$ \\
\hline C14_2 & C20_2 & $1.390(3)$ \\
\hline C15_2 & C16_2 & $1.387(3)$ \\
\hline C16_2 & C17_2 & $1.391(3)$ \\
\hline C17_2 & C18_2 & $1.509(3)$ \\
\hline C17_2 & C19_2 & $1.390(3)$ \\
\hline C19_2 & C20_2 & $1.388(3)$ \\
\hline C14_3 & C15_3 & $1.386(3)$ \\
\hline C14_3 & C20_3 & $1.390(3)$ \\
\hline C15_3 & C16_3 & $1.387(3)$ \\
\hline C16_3 & C17_3 & $1.392(3)$ \\
\hline C17_3 & C18_3 & $1.510(3)$ \\
\hline C17_3 & C19_3 & $1.390(3)$ \\
\hline C19_3 & C20_3 & $1.388(3)$ \\
\hline C14_4 & C15_4 & $1.386(3)$ \\
\hline C14_4 & C20_4 & $1.390(3)$ \\
\hline C15_4 & C16_4 & $1.387(3)$ \\
\hline C16_4 & C17_4 & $1.392(3)$ \\
\hline C17_4 & C18_4 & $1.509(3)$ \\
\hline C17_4 & C19_4 & $1.390(3)$ \\
\hline C19_4 & C20_4 & $1.389(3)$ \\
\hline C14_5 & C15_5 & $1.386(2)$ \\
\hline C14_5 & C20_5 & $1.389(2)$ \\
\hline C15_5 & C16_5 & $1.387(3)$ \\
\hline C16_5 & C17_5 & $1.391(3)$ \\
\hline C17_5 & C18_5 & $1.510(3)$ \\
\hline C17_5 & C19_5 & $1.390(3)$ \\
\hline C19_5 & C20_5 & $1.388(3)$ \\
\hline C14_6 & C15_6 & $1.385(3)$ \\
\hline C14_6 & C20_6 & $1.390(2)$ \\
\hline C15_6 & C16_6 & $1.388(3)$ \\
\hline C16_6 & C17_6 & $1.391(3)$ \\
\hline C17_6 & C18_6 & $1.510(3)$ \\
\hline C17_6 & C19_6 & $1.390(3)$ \\
\hline
\end{tabular}


Table 4: Bond Angles in ${ }^{\circ}$ for CMW04_BuTsNH2OH.

\begin{tabular}{|c|c|c|c|}
\hline Atom & Atom & Atom & Angle $/^{\circ}$ \\
\hline $\mathrm{N} 2$ & Zn1 & N1 & $80.13(9)$ \\
\hline $\mathrm{N} 2$ & $\mathrm{Zn} 1$ & N5 & $97.43(10)$ \\
\hline $\mathrm{N} 2$ & $\mathrm{Zn} 1$ & $\mathrm{~N} 4$ & $117.83(9)$ \\
\hline N3 & Zn1 & N2 & $116.20(10)$ \\
\hline N3 & Zn1 & N1 & $81.19(8)$ \\
\hline N3 & Zn1 & N5 & $112.22(9)$ \\
\hline N3 & Zn1 & N4 & $117.90(9)$ \\
\hline N5 & $\mathrm{Zn} 1$ & N1 & $165.67(9)$ \\
\hline $\mathrm{N} 4$ & $\mathrm{Zn} 1$ & N1 & $80.07(8)$ \\
\hline $\mathrm{N} 4$ & Zn1 & N5 & $88.79(9)$ \\
\hline 01 & S1 & 02 & $115.95(13)$ \\
\hline 01 & $\mathrm{~S} 1$ & N4 & $108.70(13)$ \\
\hline 01 & $\mathrm{~S} 1$ & $\mathrm{C} 7$ & $106.96(13)$ \\
\hline 02 & $\mathrm{~S} 1$ & $\mathrm{~N} 4$ & $112.45(12)$ \\
\hline 02 & $\mathrm{~S} 1$ & $\mathrm{C} 7$ & $104.84(13)$ \\
\hline $\mathrm{N} 4$ & $\mathrm{~S} 1$ & $\mathrm{C} 7$ & $107.42(13)$ \\
\hline 03 & $\mathrm{~S} 2$ & 04 & $115.56(14)$ \\
\hline 03 & $\mathrm{~S} 2$ & N3 & $113.85(13)$ \\
\hline 03 & $\mathrm{~S} 2$ & C14_5 & $105.15(11)$ \\
\hline 04 & $\mathrm{~S} 2$ & N3 & $108.69(12)$ \\
\hline 04 & $\mathrm{~S} 2$ & C14_5 & $105.56(12)$ \\
\hline N3 & $\mathrm{S} 2$ & C14_5 & $107.32(13)$ \\
\hline 05 & S3 & 06 & $116.17(15)$ \\
\hline 05 & S3 & N2 & $108.92(13)$ \\
\hline 05 & S3 & C14_6 & $105.66(13)$ \\
\hline 06 & S3 & $\mathrm{N} 2$ & $112.20(14)$ \\
\hline 06 & S3 & C14_6 & $104.75(13)$ \\
\hline N2 & S3 & C14_6 & $108.62(14)$ \\
\hline S3 & $\mathrm{N} 2$ & Zn1 & $128.44(14)$ \\
\hline C6 & N2 & Zn1 & $112.08(17)$ \\
\hline C6 & N2 & S3 & $114.71(19)$ \\
\hline $\mathrm{C} 1$ & N1 & Zn1 & $107.89(16)$ \\
\hline $\mathrm{C} 1$ & N1 & $\mathrm{C} 3$ & $112.6(2)$ \\
\hline C1 & N1 & $\mathrm{C} 5$ & $111.8(2)$ \\
\hline $\mathrm{C} 3$ & N1 & Zn1 & $105.85(15)$ \\
\hline $\mathrm{C} 5$ & N1 & Zn1 & $106.62(16)$ \\
\hline C5 & N1 & C3 & $111.7(2)$ \\
\hline S2 & N3 & Zn1 & $131.06(13)$ \\
\hline $\mathrm{C} 4$ & N3 & Zn1 & $112.49(16)$ \\
\hline $\mathrm{C} 4$ & N3 & S2 & $113.42(17)$ \\
\hline 07 & N5 & Zn1 & $127.36(18)$ \\
\hline S1 & N4 & Zn1 & $129.04(13)$ \\
\hline $\mathrm{C} 2$ & $\mathrm{~N} 4$ & Zn1 & $110.69(16)$ \\
\hline $\mathrm{C} 2$ & N4 & S1 & $114.48(18)$ \\
\hline C8 & $\mathrm{C} 7$ & S1 & $119.9(2)$ \\
\hline C8 & $\mathrm{C} 7$ & $\mathrm{C} 12$ & $119.6(3)$ \\
\hline C12 & $\mathrm{C} 7$ & S1 & $120.2(2)$ \\
\hline C9 & C8 & $\mathrm{C} 7$ & $120.0(3)$ \\
\hline C11 & $\mathrm{C} 12$ & $\mathrm{C} 7$ & $119.4(3)$ \\
\hline N1 & $\mathrm{C} 1$ & $\mathrm{C} 2$ & $109.5(2)$ \\
\hline N4 & $\mathrm{C} 2$ & $\mathrm{C} 1$ & $109.5(2)$ \\
\hline $\mathrm{N} 1$ & $\mathrm{C} 3$ & $\mathrm{C} 4$ & $111.3(2)$ \\
\hline
\end{tabular}

\begin{tabular}{|c|c|c|c|}
\hline Atom & Atom & Atom & Angle ${ }^{\circ}$ \\
\hline C11 & C10 & C13 & $120.4(3)$ \\
\hline C11 & C10 & C9 & $118.0(3)$ \\
\hline C9 & $\mathrm{C} 10$ & C13 & $121.6(3)$ \\
\hline N3 & $\mathrm{C} 4$ & C3 & $109.1(2)$ \\
\hline C12 & C11 & C10 & $121.6(3)$ \\
\hline N2 & C6 & C5 & $108.2(2)$ \\
\hline N1 & C5 & C6 & $110.6(2)$ \\
\hline C8 & C9 & C10 & $121.5(3)$ \\
\hline N2BA & Zn1B & N1B & 78.4(3) \\
\hline N2BA & Zn1B & N3B & $113.1(3)$ \\
\hline N2BA & Zn1B & N4B & $120.3(3)$ \\
\hline N2BA & Zn1B & N5B & $98.4(3)$ \\
\hline N2BB & Zn1B & N1B & $81.3(3)$ \\
\hline N2BB & Zn1B & N5B & $97.2(3)$ \\
\hline N3B & Zn1B & N1B & $79.57(9)$ \\
\hline N3B & Zn1B & N2BB & $120.6(3)$ \\
\hline N3B & Zn1B & N5B & $90.22(10)$ \\
\hline N4B & Zn1B & N1B & $80.14(9)$ \\
\hline N4B & Zn1B & N2BB & $114.5(3)$ \\
\hline N4B & Zn1B & N3B & $116.65(9)$ \\
\hline N4B & Zn1B & N5B & $112.05(10)$ \\
\hline N5B & Zn1B & N1B & 166.89(9) \\
\hline 01B & S1B & N3B & 108.33(13) \\
\hline 01B & S1B & C7B & $106.16(13)$ \\
\hline O2B & S1B & 01B & $116.00(13)$ \\
\hline O2B & S1B & N3B & $112.72(13)$ \\
\hline O2B & S1B & C7B & $105.20(14)$ \\
\hline N3B & S1B & C7B & $107.90(14)$ \\
\hline O3B & S2B & O4B & $115.71(15)$ \\
\hline O3B & S2B & N4B & 113.71(13) \\
\hline O3B & S2B & C14_1 & $107.4(2)$ \\
\hline O3B & S2B & C14_2 & $102.9(3)$ \\
\hline O4B & S2B & N4B & $109.11(12)$ \\
\hline O4B & S2B & C14_1 & $103.0(4)$ \\
\hline O4B & S2B & C14_2 & $107.0(4)$ \\
\hline N4B & S2B & C14_1 & $106.9(4)$ \\
\hline N4B & S2B & C14_2 & $107.7(5)$ \\
\hline 05BA & S3BA & 06BA & 113.8(11) \\
\hline 05BA & S3BA & N2BA & $109.8(11)$ \\
\hline 05BA & S3BA & C14_3 & 106.2(9) \\
\hline 06BA & S3BA & N2BA & $112.7(9)$ \\
\hline 06BA & S3BA & C14_3 & 105.8(7) \\
\hline N2BA & S3BA & C14_3 & 108.1(6) \\
\hline 05BB & S3BB & N2BB & $107.8(10)$ \\
\hline 05BB & S3BB & C14_4 & $105.4(8)$ \\
\hline O6BB & S3BB & O5BB & $118.4(10)$ \\
\hline 06BB & S3BB & N2BB & $111.2(9)$ \\
\hline 06BВ & S3BB & C14_4 & $104.6(6)$ \\
\hline N2BB & S3BB & C14_4 & $109.0(6)$ \\
\hline C3B & N1B & Zn1B & 107.45(17) \\
\hline C5B & N1B & Zn1B & $105.96(16)$ \\
\hline C5B & N1B & C3B & $112.5(2)$ \\
\hline
\end{tabular}




\begin{tabular}{|c|c|c|c|}
\hline Atom & Atom & Atom & Angle $/{ }^{\circ}$ \\
\hline C5B & N1B & $\mathrm{C} 1 \mathrm{~B}$ & $112.0(2)$ \\
\hline C1B & N1B & Zn1B & $106.10(17)$ \\
\hline C1B & N1B & C3B & $112.3(2)$ \\
\hline S3BA & $\mathrm{N} 2 \mathrm{BA}$ & $\mathrm{Zn} 1 \mathrm{~B}$ & $128.4(8)$ \\
\hline C2B & N2BA & Zn1B & $113.9(5)$ \\
\hline C2B & N2BA & S3BA & $109.3(8)$ \\
\hline S3BB & N2BB & $\mathrm{Zn} 1 \mathrm{~B}$ & $128.4(8)$ \\
\hline C2B & N2BB & Zn1B & $109.3(4)$ \\
\hline $\mathrm{C} 2 \mathrm{~B}$ & N2BB & S3BB & $120.5(9)$ \\
\hline S1B & N3B & $\mathrm{Zn1B}$ & $129.11(13)$ \\
\hline $\mathrm{C} 4 \mathrm{~B}$ & N3B & $\mathrm{Zn} 1 \mathrm{~B}$ & $111.52(19)$ \\
\hline C4B & N3B & S1B & $114.4(2)$ \\
\hline S2B & N4B & Zn1B & $130.65(14)$ \\
\hline C6B & N4B & $\mathrm{Zn} 1 \mathrm{~B}$ & $113.52(17)$ \\
\hline C6B & N4B & $\mathrm{S} 2 \mathrm{~B}$ & $113.51(18)$ \\
\hline 07B & N5B & Zn1B & $128.28(17)$ \\
\hline N2BA & $\mathrm{C} 2 \mathrm{~B}$ & C1B & $103.5(4)$ \\
\hline N2BB & $\mathrm{C} 2 \mathrm{~B}$ & $\mathrm{C} 1 \mathrm{~B}$ & $113.7(4)$ \\
\hline N1B & $\mathrm{C} 3 \mathrm{~B}$ & $\mathrm{C} 4 \mathrm{~B}$ & $109.5(2)$ \\
\hline N3B & $\mathrm{C} 4 \mathrm{~B}$ & $\mathrm{C} 3 \mathrm{~B}$ & $109.5(2)$ \\
\hline N1B & C5B & $\mathrm{C} 6 \mathrm{~B}$ & $111.0(2)$ \\
\hline N1B & C1B & $\mathrm{C} 2 \mathrm{~B}$ & $110.4(2)$ \\
\hline N4B & C6B & C5B & $108.8(2)$ \\
\hline C8B & C7B & S1B & $119.3(2)$ \\
\hline $\mathrm{C} 12 \mathrm{~B}$ & C7B & S1B & $120.7(2)$ \\
\hline C12B & C7B & C8B & $119.9(3)$ \\
\hline C9B & C8B & C7B & 119.7(3) \\
\hline $\mathrm{C} 8 \mathrm{~B}$ & C9B & C10B & $121.4(3)$ \\
\hline C9B & C10B & C13B & $121.4(3)$ \\
\hline $\mathrm{C} 11 \mathrm{~B}$ & C10B & C9B & $117.9(3)$ \\
\hline C11B & C10B & C13B & $120.7(3)$ \\
\hline C12B & C11B & C10B & $121.5(3)$ \\
\hline C7B & C12B & C11B & $119.6(3)$ \\
\hline C32 & N7 & C28 & $108.3(2)$ \\
\hline C32 & N7 & C36 & $108.9(2)$ \\
\hline C32 & N7 & $\mathrm{C} 40$ & $112.0(2)$ \\
\hline C36 & N7 & $\mathrm{C} 28$ & $111.3(2)$ \\
\hline C36 & N7 & $\mathrm{C} 40$ & $107.6(2)$ \\
\hline $\mathrm{C} 40$ & N7 & $\mathrm{C} 28$ & $108.7(2)$ \\
\hline N7 & C28 & C29 & $115.3(2)$ \\
\hline C30 & $\mathrm{C} 29$ & $\mathrm{C} 28$ & $110.7(3)$ \\
\hline C31 & C30 & $\mathrm{C} 29$ & $113.0(3)$ \\
\hline C33 & C32 & N7 & $115.0(2)$ \\
\hline C32 & C33 & C34 & $111.1(2)$ \\
\hline C35 & C34 & C33 & $111.8(3)$ \\
\hline C37 & C36 & N7 & $115.1(2)$ \\
\hline C36 & C37 & C38 & $110.9(2)$ \\
\hline C39 & C38 & $\mathrm{C} 37$ & $114.4(3)$ \\
\hline C41 & C40 & N7 & $116.6(2)$ \\
\hline C40 & C41 & C42 & 108.1(2) \\
\hline C41 & C42 & C43 & $113.0(3)$ \\
\hline $\mathrm{C} 21 \mathrm{~A}$ & N7A & C25A & 109.8(3) \\
\hline C21A & N7A & C29A & $110.3(3)$ \\
\hline $\mathrm{C} 21 \mathrm{~A}$ & N7A & C33A & 108.3(3) \\
\hline $\mathrm{C} 25 \mathrm{~A}$ & N7A & C29A & $110.5(3)$ \\
\hline $\mathrm{C} 25 \mathrm{~A}$ & N7A & C33A & $109.8(3)$ \\
\hline $\mathrm{C} 29 \mathrm{~A}$ & N7A & C33A & 108.1(3) \\
\hline C22A & $\mathrm{C} 21 \mathrm{~A}$ & N7A & $117.3(3)$ \\
\hline
\end{tabular}

\begin{tabular}{|c|c|c|c|}
\hline Atom & Atom & Atom & Angle $/^{\circ}$ \\
\hline$\overline{\mathrm{C} 21 \mathrm{~A}}$ & $\mathrm{C} 22 \mathrm{~A}$ & C23A & $109.6(4)$ \\
\hline $\mathrm{C} 24 \mathrm{~A}$ & $\mathrm{C} 23 \mathrm{~A}$ & $\mathrm{C} 22 \mathrm{~A}$ & $114.7(4)$ \\
\hline N7A & C25A & $\mathrm{C} 26 \mathrm{~A}$ & $115.2(3)$ \\
\hline C27A & C26A & C25A & $110.7(4)$ \\
\hline C28A & C27A & C26A & $114.3(5)$ \\
\hline C30A & C29A & N7A & $117.5(3)$ \\
\hline C29A & C30A & C31A & $109.9(4)$ \\
\hline C32A & C31A & C30A & $112.0(6)$ \\
\hline C34A & C33A & N7A & $114.6(3)$ \\
\hline C33A & C34A & C35A & 111.6(3) \\
\hline C36A & C35A & C34A & 110.1(3) \\
\hline C21B & N7B & C25B & $110.0(3)$ \\
\hline $\mathrm{C} 21 \mathrm{~B}$ & N7B & C29B & $110.7(3)$ \\
\hline $\mathrm{C} 21 \mathrm{~B}$ & N7B & C33B & 108.2(3) \\
\hline C25B & N7B & C29B & 110.1(3) \\
\hline C25B & N7B & C33B & 109.6(3) \\
\hline C29B & N7B & C33B & 108.1(3) \\
\hline $\mathrm{C} 22 \mathrm{~B}$ & $\mathrm{C} 21 \mathrm{~B}$ & N7B & $118.1(3)$ \\
\hline $\mathrm{C} 21 \mathrm{~B}$ & $\mathrm{C} 22 \mathrm{~B}$ & C23B & $109.5(4)$ \\
\hline C24B & C23B & C22B & $114.7(4)$ \\
\hline N7B & C25B & C26B & $114.9(3)$ \\
\hline C27B & C26B & C25B & $110.9(4)$ \\
\hline C28B & C27B & C26B & $114.4(5)$ \\
\hline C30B & C29B & N7B & $117.7(3)$ \\
\hline C29B & C30B & C31B & $110.0(4)$ \\
\hline C32B & C31B & C30B & $112.0(6)$ \\
\hline C34B & C33B & N7B & 114.4(3) \\
\hline C33B & C34B & C35B & 111.6(3) \\
\hline C36B & C35B & C34B & $110.2(3)$ \\
\hline C15_1 & C14_1 & S2B & $117.4(4)$ \\
\hline C15_1 & C14_1 & C20_1 & $119.77(19)$ \\
\hline C20_1 & C14_1 & S2B & $122.7(4)$ \\
\hline C14_1 & C15_1 & C16_1 & 119.73(19) \\
\hline C15_1 & C16_1 & C17_1 & 121.1(2) \\
\hline C16_1 & C17_1 & C18_1 & $120.6(2)$ \\
\hline C19_1 & C17_1 & C16_1 & 118.57(19) \\
\hline C19_1 & C17_1 & C18_1 & $120.8(2)$ \\
\hline C20_1 & C19_1 & C17_1 & $120.7(2)$ \\
\hline C19_1 & C20_1 & C14_1 & $120.0(2)$ \\
\hline C15_2 & C14_2 & S2B & $121.0(4)$ \\
\hline C15_2 & C14_2 & C20_2 & $119.77(19)$ \\
\hline C20_2 & C14_2 & S2B & $119.2(4)$ \\
\hline C14_2 & C15_2 & C16_2 & $119.70(19)$ \\
\hline C15_2 & C16_2 & C17_2 & $121.2(2)$ \\
\hline C16_2 & C17_2 & C18_2 & $120.7(2)$ \\
\hline C19_2 & C17_2 & C16_2 & $118.56(19)$ \\
\hline C19_2 & C17_2 & C18_2 & $120.7(2)$ \\
\hline C20_2 & C19_2 & C17_2 & $120.6(2)$ \\
\hline C19_2 & C20_2 & C14_2 & 120.1(2) \\
\hline C15_3 & C14_3 & S3BA & 121.4(5) \\
\hline C15_3 & C14_3 & C20_3 & $119.77(19)$ \\
\hline C20_3 & C14_3 & S3BA & $118.8(5)$ \\
\hline C14_3 & C15_3 & C16_3 & $119.74(19)$ \\
\hline C15_3 & C16_3 & C17_3 & $121.2(2)$ \\
\hline C16_3 & C17_3 & C18_3 & $120.7(2)$ \\
\hline C19_3 & C17_3 & C16_3 & 118.53(19) \\
\hline C19_3 & C17_3 & C18_3 & $120.7(2)$ \\
\hline C20_3 & C19_3 & C17_3 & $120.7(2)$ \\
\hline
\end{tabular}




\begin{tabular}{|c|c|c|c|c|c|c|c|}
\hline Atom & Atom & Atom & Angle ${ }^{\circ}$ & Atom & Atom & Atom & Angle $/^{\circ}$ \\
\hline$\overline{\text { C19_3 }}$ & C20_3 & C14_3 & $120.1(2)$ & $\overline{C 16 \_5}$ & C17_5 & C18_5 & $120.8(2)$ \\
\hline C15_4 & C14_4 & S3BB & $121.3(5)$ & C19_5 & C17_5 & C16_5 & $118.58(18)$ \\
\hline C15_4 & C14_4 & C20_4 & $119.74(19)$ & C19_5 & C17_5 & C18_5 & $120.63(19)$ \\
\hline C20_4 & C14_4 & S3BB & $118.9(5)$ & C20_5 & C19_5 & C17_5 & $120.64(18)$ \\
\hline C14_4 & C15_4 & C16_4 & $119.7(2)$ & C19_5 & C20_5 & C14_5 & $120.10(19)$ \\
\hline C15_4 & C16_4 & C17_4 & $121.1(2)$ & C15_6 & C14_6 & S3 & $120.39(17)$ \\
\hline C16_4 & C17_4 & C18_4 & $120.7(2)$ & C15_6 & C14_6 & C20_6 & $119.77(18)$ \\
\hline C19_4 & C17_4 & C16_4 & $118.5(2)$ & C20_6 & C14_6 & S3 & $119.83(18)$ \\
\hline C19_4 & C17_4 & C18_4 & $120.7(2)$ & C14_6 & C15_6 & C16_6 & $119.74(19)$ \\
\hline C20_4 & C19_4 & C17_4 & $120.6(2)$ & C15_6 & C16_6 & C17_6 & 121.1(2) \\
\hline C19_4 & C20_4 & C14_4 & $120.0(2)$ & C16_6 & C17_6 & C18_6 & $120.6(2)$ \\
\hline C15_5 & C14_5 & $\mathrm{S} 2$ & $120.09(15)$ & C19_6 & C17_6 & C16_6 & $118.57(18)$ \\
\hline C15_5 & C14_5 & C20_5 & $119.82(17)$ & C19_6 & C17_6 & C18_6 & $120.8(2)$ \\
\hline C20_5 & C14_5 & $\mathrm{S} 2$ & $120.09(16)$ & C20_6 & C19_6 & C17_6 & $120.70(19)$ \\
\hline C14_5 & C15_5 & C16_5 & $119.66(18)$ & C19_6 & C20_6 & C14_6 & $120.0(2)$ \\
\hline C15_5 & C16_5 & C17_5 & $121.2(2)$ & & & & \\
\hline
\end{tabular}

Table 5: Hydrogen Fractional Atomic Coordinates $\left(\times 10^{4}\right)$ and Equivalent Isotropic Displacement Parameters $\left(\AA^{2} \times 10^{3}\right)$ for CMW04_BuTsNH2OH. $U_{e q}$ is defined as $1 / 3$ of the trace of the orthogonalised $U_{i j}$.

\begin{tabular}{|c|c|c|c|c|}
\hline Atom & & & & $U_{e q}$ \\
\hline$\overline{\mathrm{H}}$ & 4468 & 4245 & 3050 & 53 \\
\hline H5A & 6025 & 5454 & 3557 & 27 \\
\hline H5B & 5073 & 5606 & 3249 & 27 \\
\hline H8 & 3378 & 5289 & 1412 & 24 \\
\hline H12 & 4132 & 7594 & 1289 & 28 \\
\hline $\mathrm{H} 1 \mathrm{~A}$ & 5792 & 6541 & 951 & 18 \\
\hline H1B & 7029 & 7101 & 1050 & 18 \\
\hline $\mathrm{H} 2 \mathrm{~A}$ & 7111 & 7364 & 2212 & 17 \\
\hline H2B & 6090 & 7519 & 1796 & 17 \\
\hline H3A & 6927 & 5155 & 948 & 18 \\
\hline H3B & 6520 & 5704 & 451 & 18 \\
\hline $\mathrm{H} 4 \mathrm{~A}$ & 4816 & 5215 & 714 & 19 \\
\hline $\mathrm{H} 4 \mathrm{~B}$ & 5111 & 4486 & 527 & 19 \\
\hline H11 & 3297 & 7362 & 142 & 31 \\
\hline H6A & 8091 & 5254 & 1892 & 26 \\
\hline H6B & 9028 & 5947 & 2376 & 26 \\
\hline H13D & 2028 & 6463 & -786 & 43 \\
\hline H13E & 1728 & 5554 & -743 & 43 \\
\hline $\mathrm{H} 13 \mathrm{~F}$ & 2851 & 6072 & -933 & 43 \\
\hline $\mathrm{H} 5 \mathrm{C}$ & 8252 & 6832 & 1968 & 21 \\
\hline H5D & 8368 & 6379 & 1319 & 21 \\
\hline H9 & 2520 & 5071 & 278 & 27 \\
\hline H7B & -675 & 9005 & 3002 & 38 \\
\hline H5BA & 777 & 10213 & 3573 & 24 \\
\hline H5BB & -160 & 10373 & 3246 & 24 \\
\hline H2BA & 2956 & 10108 & 1987 & 33 \\
\hline $\mathrm{H} 2 \mathrm{BB}$ & 3922 & 10771 & 2488 & 33 \\
\hline H2BC & 3036 & 10138 & 1963 & 33 \\
\hline $\mathrm{H} 2 \mathrm{BD}$ & 3920 & 10800 & 2496 & 33 \\
\hline Н3BA & 826 & 11498 & 1021 & 27 \\
\hline H3BB & 2070 & 12052 & 1175 & 27 \\
\hline H4BA & 2046 & 12230 & 2334 & 26 \\
\hline H4BB & 1060 & 12416 & 1906 & 26 \\
\hline H5BC & 1926 & 10097 & 987 & 27 \\
\hline H5BD & 1577 & 10686 & 511 & 27 \\
\hline
\end{tabular}




\begin{tabular}{|c|c|c|c|c|c|}
\hline Atom & & & & & $\boldsymbol{U}_{e q}$ \\
\hline$\overline{\mathrm{H} 1 \mathrm{BA}}$ & 3221 & 11712 & 2089 & 31 & \\
\hline H1BB & 3379 & 11279 & 1443 & 31 & \\
\hline H6BA & -166 & 10197 & 723 & 25 & \\
\hline H6BB & 114 & 9470 & 508 & 25 & \\
\hline H8B & -1693 & 10197 & 1393 & 28 & \\
\hline H9B & -2538 & 10036 & 255 & 29 & \\
\hline H11B & -1719 & 12343 & 213 & 25 & \\
\hline H12B & -895 & 12514 & 1359 & 25 & \\
\hline H13A & -2198 & 11154 & -914 & 43 & \\
\hline H13B & -3095 & 11448 & -727 & 43 & \\
\hline H13C & -3283 & 10548 & -742 & 43 & \\
\hline H28A & 4785 & 918 & 6094 & 24 & \\
\hline H28B & 4840 & 1762 & 6308 & 24 & \\
\hline H29A & 4123 & 1100 & 4925 & 31 & \\
\hline H29B & 4326 & 1974 & 5166 & 31 & \\
\hline H30A & 5858 & 2105 & 4802 & 41 & \\
\hline H30B & 6209 & 2096 & 5608 & 41 & \\
\hline H31A & 5643 & 817 & 4652 & 57 & \\
\hline H31B & 5943 & 783 & 5458 & 57 & \\
\hline H31C & 6833 & 1302 & 5059 & 57 & \\
\hline H32A & 2762 & 237 & 5212 & 20 & \\
\hline H32B & 3171 & -149 & 5860 & 20 & \\
\hline H33A & 1144 & 40 & 5576 & 22 & \\
\hline H33B & 1558 & -294 & 6260 & 22 & \\
\hline H34A & 1195 & -1017 & 4921 & 31 & \\
\hline H34B & 1596 & -1352 & 5607 & 31 & \\
\hline H35A & -271 & -1995 & 5211 & 50 & \\
\hline H35B & -445 & -1193 & 5281 & 50 & \\
\hline H35C & -45 & -1559 & 5947 & 50 & \\
\hline H36A & 2595 & 1474 & 5330 & 22 & \\
\hline H36B & 1925 & 1240 & 5925 & 22 & \\
\hline H37A & 3870 & 2553 & 6020 & 27 & \\
\hline H37B & 3248 & 2310 & 6639 & 27 & \\
\hline H38A & 2863 & 3312 & 5965 & 30 & \\
\hline H38B & 2212 & 2645 & 5369 & 30 & \\
\hline H39A & 1027 & 2882 & 5987 & 46 & \\
\hline H39B & 1645 & 2675 & 6672 & 46 & \\
\hline H39C & 988 & 2018 & 6067 & 46 & \\
\hline $\mathrm{H} 40 \mathrm{~A}$ & 3746 & 1433 & 7085 & 19 & \\
\hline $\mathrm{H} 40 \mathrm{~B}$ & 2557 & 801 & 6925 & 19 & \\
\hline H41A & 4361 & 333 & 7030 & 22 & \\
\hline H41B & 3158 & -197 & 7091 & 22 & \\
\hline $\mathrm{H} 42 \mathrm{~A}$ & 3365 & 592 & 8112 & 29 & \\
\hline H42B & 4594 & 1044 & 8063 & 29 & \\
\hline H43A & 4934 & -119 & 8225 & 43 & \\
\hline H43B & 3711 & -555 & 8296 & 43 & \\
\hline H43C & 4508 & 114 & 8858 & 43 & \\
\hline H21A & 1954 & 5062 & 3321 & 24 & \\
\hline H21B & 2652 & 4548 & 3238 & 24 & \\
\hline H22A & 440 & 4138 & 2653 & 44 & \\
\hline H22B & 1099 & 3584 & 2596 & 44 & \\
\hline H23A & 1721 & 5092 & 2123 & 46 & \\
\hline H23B & 2243 & 4469 & 2003 & 46 & \\
\hline H24A & 1174 & 4457 & 1002 & 66 & \\
\hline H24B & 200 & 4434 & 1359 & 66 & \\
\hline $\mathrm{H} 24 \mathrm{C}$ & 523 & 3690 & 1324 & 66 & \\
\hline H25A & 3274 & 4660 & 4430 & 26 & \\
\hline H25B & 2468 & 4344 & 4942 & 26 & \\
\hline
\end{tabular}




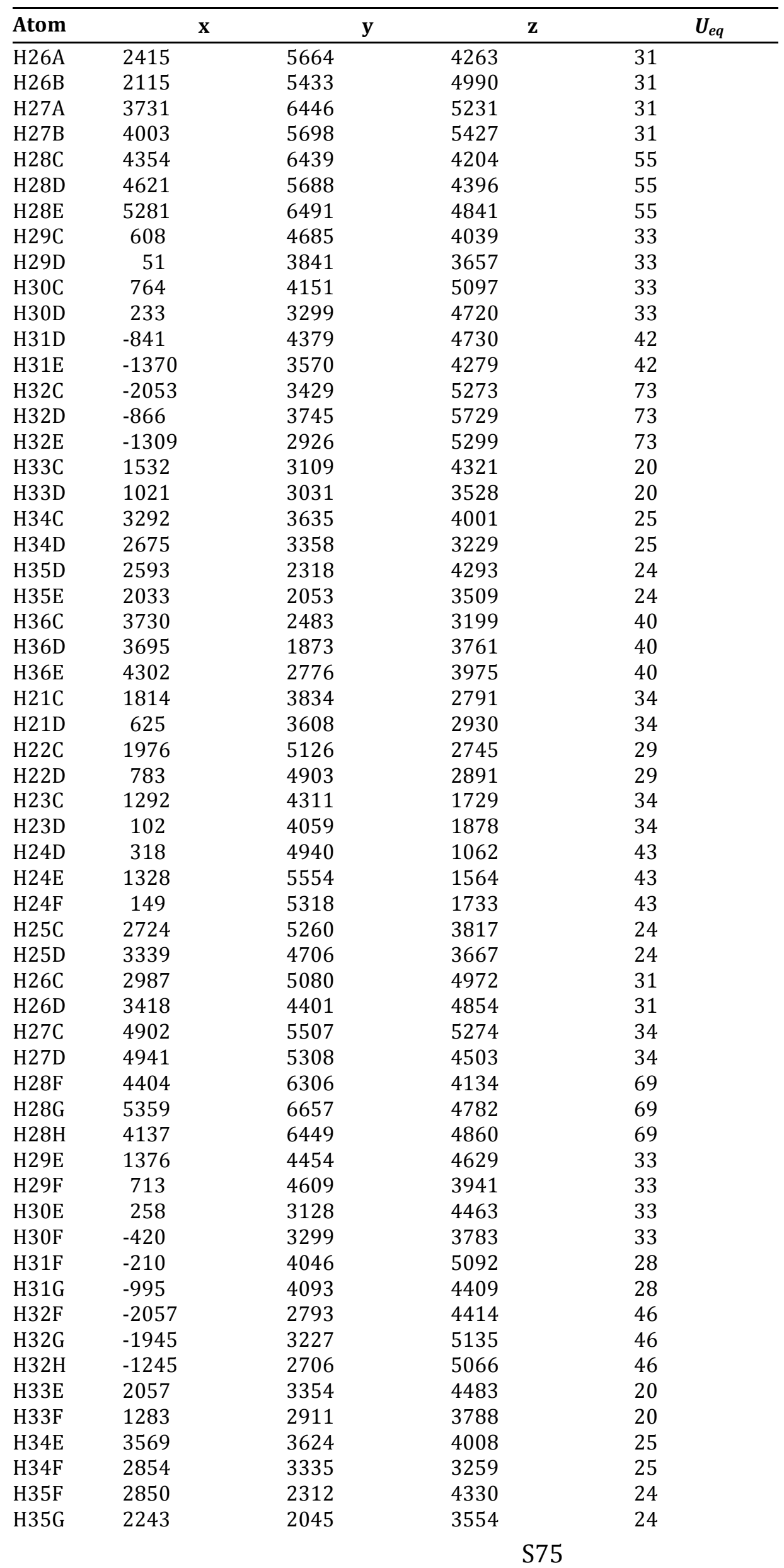




\begin{tabular}{|c|c|c|c|c|c|}
\hline Atom & & & & & $U_{e q}$ \\
\hline$\overline{\mathrm{H} 36 \mathrm{~F}}$ & 3964 & 2527 & 3223 & 31 & \\
\hline H36G & 3837 & 1820 & 3692 & 31 & \\
\hline $\mathrm{H} 36 \mathrm{H}$ & 4517 & 2685 & 4013 & 31 & \\
\hline H15_1 & 744 & 8152 & 1921 & 23 & \\
\hline H16_1 & 1204 & 7145 & 1549 & 24 & \\
\hline H18A_1 & 967 & 6117 & 773 & 47 & \\
\hline H18B_1 & 584 & 6232 & -9 & 47 & \\
\hline H18C_1 & -262 & 5687 & 406 & 47 & \\
\hline H19_1 & -1019 & 6773 & -183 & 21 & \\
\hline H20_1 & -1484 & 7780 & 185 & 22 & \\
\hline H15_2 & 697 & 8112 & 1811 & 23 & \\
\hline H16_2 & 1034 & 7133 & 1272 & 22 & \\
\hline H18A_2 & 797 & 6315 & 273 & 37 & \\
\hline H18B_2 & -17 & 6336 & -405 & 37 & \\
\hline H18C_2 & -454 & 5798 & 177 & 37 & \\
\hline H19_2 & -1299 & 7004 & -344 & 17 & \\
\hline H20_2 & -1645 & 7981 & 194 & 15 & \\
\hline H15_3 & 1156 & 8813 & 3631 & 26 & \\
\hline H16_3 & 765 & 7499 & 3410 & 29 & \\
\hline H18A_3 & 2212 & 6593 & 3244 & 46 & \\
\hline H18B_3 & 2037 & 6649 & 2437 & 46 & \\
\hline H18C_3 & 1040 & 6461 & 2814 & 46 & \\
\hline H19_3 & 3389 & 7980 & 2558 & 27 & \\
\hline H20_3 & 3777 & 9297 & 2768 & 24 & \\
\hline H15_4 & 1011 & 8707 & 3638 & 26 & \\
\hline H16_4 & 547 & 7377 & 3462 & 24 & \\
\hline H18A_4 & 1154 & 6303 & 3316 & 41 & \\
\hline H18B_4 & 2206 & 6440 & 3004 & 41 & \\
\hline H18C_4 & 1096 & 6357 & 2514 & 41 & \\
\hline H19_4 & 3136 & 7724 & 2603 & 27 & \\
\hline H20_4 & 3645 & 9055 & 2816 & 25 & \\
\hline H15_5 & 5725 & 3174 & 1888 & 27 & \\
\hline H16_5 & 6041 & 2158 & 1394 & 30 & \\
\hline H18A_5 & 5799 & 1305 & 409 & 44 & \\
\hline H18B_5 & 4930 & 1268 & -259 & 44 & \\
\hline H18C_5 & 4560 & 779 & 366 & 44 & \\
\hline H19_5 & 3650 & 1935 & -212 & 22 & \\
\hline H20_5 & 3320 & 2946 & 282 & 19 & \\
\hline H15_6 & 6295 & 3940 & 3603 & 43 & \\
\hline H16_6 & 5861 & 2611 & 3454 & 49 & \\
\hline H18A_6 & 6756 & 1585 & 3345 & 78 & \\
\hline H18B_6 & 7386 & 1688 & 2728 & 78 & \\
\hline H18C_6 & 6128 & 1540 & 2579 & 78 & \\
\hline H19_6 & 8323 & 2959 & 2469 & 44 & \\
\hline H20_6 & 8785 & 4291 & 2638 & 39 & \\
\hline
\end{tabular}


Submitted by: Christian Wallen

Emory University

Solved by: John Bacsa

Sample ID: 1-H202

\section{Crystal Data and Experimental}

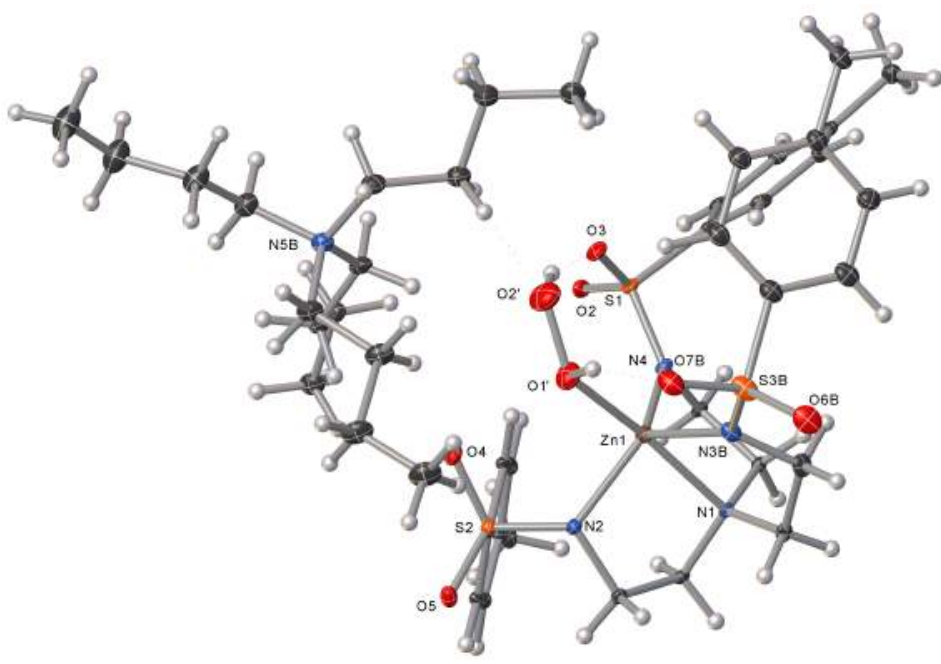

Experimental. Single colourless prism-shaped crystals of (CMW04-BuZnTs02H2xtal2) were recrystallised from a mixture of diethyl ether and THF by vapor diffusion. A suitable crystal $(0.78 \times 0.44 \times 0.40 \mathrm{~mm})$ was selected and mounted on a loop with paratone oil on a Bruker APEX-II CCD diffractometer. The crystal was cooled to $T=$ 100(2) K during data collection. The structure was solved with the ShelXT2e structure solution program using combined Patterson and dual-space recycling methods and by using Olex2 ${ }^{2 c}$ as the graphical interface. The crystal structure was refined with version of ShelXL ${ }^{2 b}$ using Least Squares minimisation.

Crystal Data. $\mathrm{C}_{86} \mathrm{H}_{142} \mathrm{~N}_{10} \mathrm{O}_{15} \mathrm{~S}_{6} \mathrm{Zn}_{2}, M_{r}=1879.19$, triclinic, P-1 (No. 2), $a=9.3583(12) \AA, b=13.4896(18) \AA, c=$ 19.634(3) $\AA, \quad \alpha=92.9514(19)^{\circ}, \quad \beta=97.9613(18)^{\circ}, \quad \gamma=$ 109.1389(19) ${ }^{\circ}, V=2306.5(5) \AA^{3}, T=100(2) \mathrm{K}, Z=1, Z^{\prime}=$ 0.5, $\mu\left(\mathrm{MoK}_{a}\right)=0.723,30794$ reflections measured, 14175 unique $\left(R_{\text {int }}=0.0408\right)$ which were used in all calculations. The final $w R_{2}$ was 0.2157 (all data) and $R_{1}$ was 0.0864 (I > 2(I)).

\begin{tabular}{|c|c|}
\hline Compound & $\begin{array}{l}\text { CMW04- } \\
\text { BuZnTs02H2xtal2 }\end{array}$ \\
\hline Formula & $\mathrm{C}_{86} \mathrm{H}_{142} \mathrm{~N}_{10} \mathrm{O}_{15} \mathrm{~S}_{6} \mathrm{Zn}_{2}$ \\
\hline$D_{\text {calc. }} / \mathrm{g} \mathrm{cm}^{-3}$ & 1.353 \\
\hline$\mu / \mathrm{mm}^{-1}$ & 0.723 \\
\hline Formula Weight & 1879.19 \\
\hline Colour & colourless \\
\hline Shape & prism \\
\hline Max Size/mm & 0.78 \\
\hline Mid Size/mm & 0.44 \\
\hline Min Size/mm & 0.40 \\
\hline $\mathrm{T} / \mathrm{K}$ & $100(2)$ \\
\hline Crystal System & triclinic \\
\hline Space Group & $\mathrm{P}-1$ \\
\hline$a / \AA$ & $9.3583(12)$ \\
\hline$b / \AA$ & $13.4896(18)$ \\
\hline$c / \AA$ & $19.634(3)$ \\
\hline$\alpha /^{\circ}$ & 92.9514(19) \\
\hline$\beta /^{\circ}$ & $97.9613(18)$ \\
\hline$\gamma /^{\circ}$ & $109.1389(19)$ \\
\hline $\mathrm{V} / \AA^{3}$ & $2306.5(5)$ \\
\hline$Z^{\prime}$ & 1 \\
\hline$Z^{\prime}$ & 0.5 \\
\hline$\Theta_{\min } /^{\circ}$ & 1.827 \\
\hline$\Theta_{\max } /^{\circ}$ & 31.162 \\
\hline Measured Refl. & 30794 \\
\hline Independent Refl. & 14175 \\
\hline$I>2 \sigma(I)$ & 10044 \\
\hline$R_{\text {int }}$ & 0.0408 \\
\hline Parameters & 662 \\
\hline Restraints & 495 \\
\hline Largest Peak & 2.569 \\
\hline Deepest Hole & -1.172 \\
\hline GooF & 1.066 \\
\hline$w R_{2}$ (all data) & 0.2157 \\
\hline$w R_{2}$ & 0.1938 \\
\hline$R_{1}$ (all data) & 0.1204 \\
\hline$R_{1}$ & 0.0864 \\
\hline CCDC number & 1429663 \\
\hline
\end{tabular}


Structure Quality Indicators

Reflections:

d min

$0.69^{1 / \sigma}$

9.3 Rint

$4.08 \%$ complete

$95 \%$

Refinement:

Shift

0.001 Max Peak

2.6 Min Peak

$-1.2^{\mathrm{GooF}}$

1.066

A colourless prism-shaped crystal with dimensions $0.78 \times 0.44 \times 0.40 \mathrm{~mm}$ was mounted on a loop with paratone oil. X-ray diffraction data were collected using a Bruker APEX-II CCD diffractometer equipped with an Oxford Cryosystems low-temperature apparatus operating at $T=100(2) \mathrm{K}$.

Data were measured using $\phi$ and $\omega$ scans with $\mathrm{MoK}_{\alpha}$ radiation (fine-focus sealed tube, $45 \mathrm{kV}, 35 \mathrm{~mA}$ ). The total number of runs and images was based on the strategy calculation from the program APEX2 (Bruker) ${ }^{2 a}$. The maximum resolution achieved was $\Theta=31.162^{\circ}$.

Unit cell indexing was performed by using the APEX2 (Bruker) ${ }^{2 a}$ software and refined using SAINT (Bruker) $^{2 \mathrm{~d}}$ on 9973 reflections, 32 percent of the observed reflections. Data reduction, scaling and absorption corrections were performed using SAINT (Bruker) ${ }^{2 \mathrm{~d}}$ and SADABS-2014/5 (Bruker)2a,d was used for absorption correction. $w R_{2}$ (int) was 0.1252 before and 0.0520 after correction. The Ratio of minimum to maximum transmission is 0.8144 .The $\lambda / 2$ correction factor is 0.00150 . The software also corrects for Lorentz polarisation. The final completeness is 99.70 out to 31.162 in $\Theta$. The absorption coefficient $(\mu)$ of this material is $0.723 \mathrm{~mm}$ and the minimum and maximum transmissions are 0.6077 and 0.7462 .

The structure was solved in the space group P1 with the ShelXT2e structure solution program using combined Patterson and dual-space recycling methods. The space group P-1 (\# 2) was determined by ShelXT$^{2 e}$ structure solution program. The crystal structure was refined by Least Squares using version 2014/7 of ShelXL. ${ }^{2 b}$ All non-hydrogen atoms were refined anisotropically. Hydrogen atom positions were calculated geometrically and refined using the riding model.

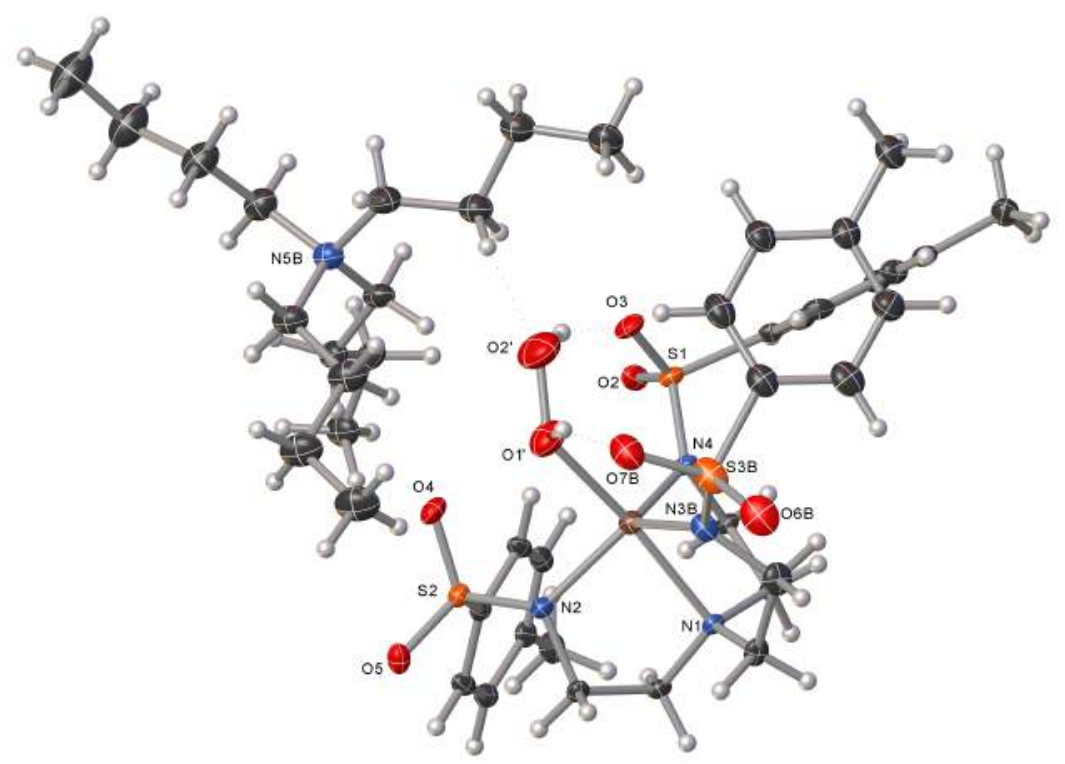

Figure 1: Plot of $1-\mathrm{H}_{2} \mathrm{O}_{2}$, one of the two disorder components of $1-\mathrm{H}_{2} \mathrm{O}_{2} / 1-\mathrm{OH}_{2}$. 


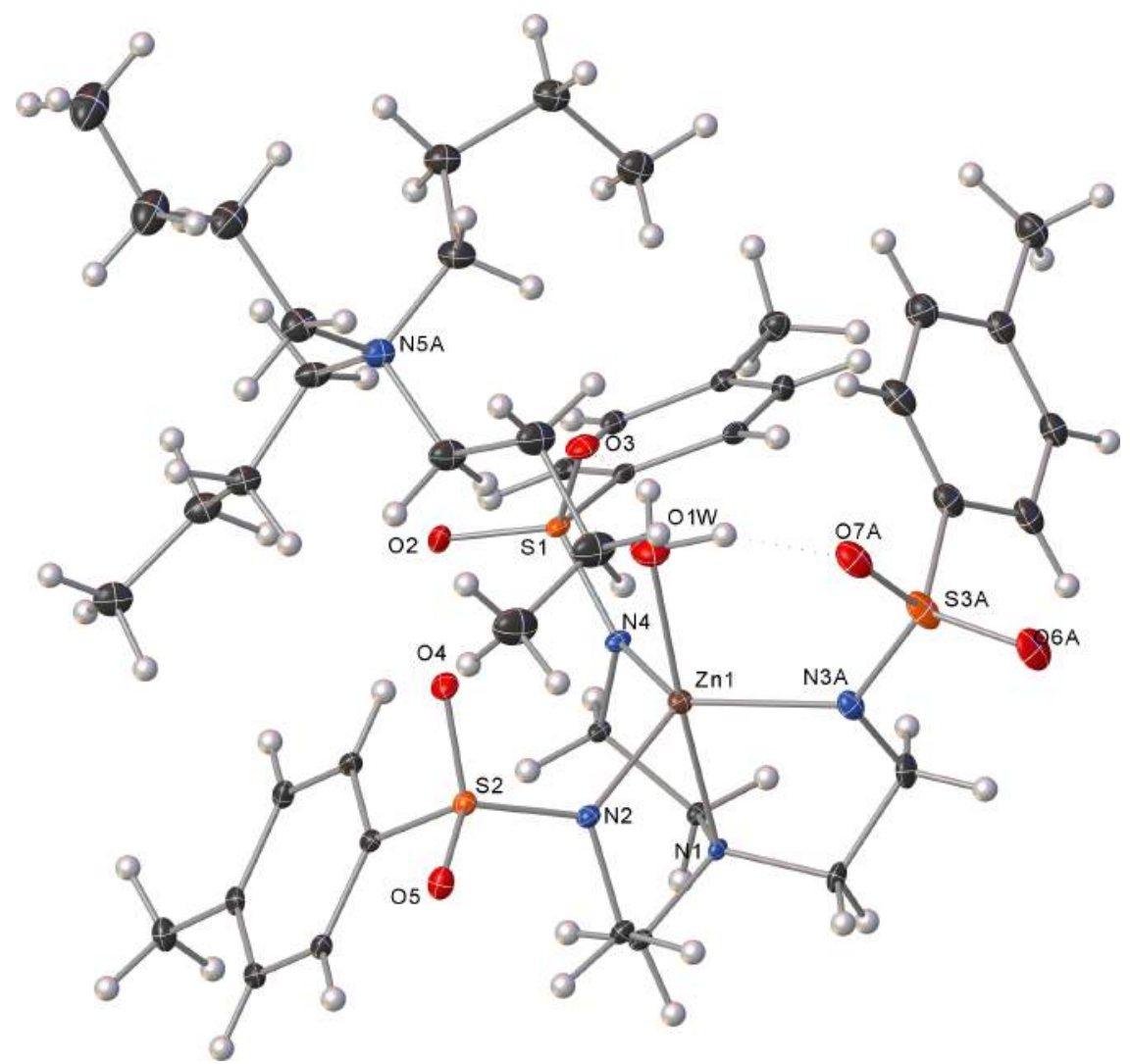

Figure 2: Plot of 1- $\mathrm{OH}_{2}$, one of the two disorder components of $1-\mathrm{H}_{2} \mathrm{O}_{2} / 1-\mathrm{OH}_{2}$.

\section{Data Plots: Diffraction Data}
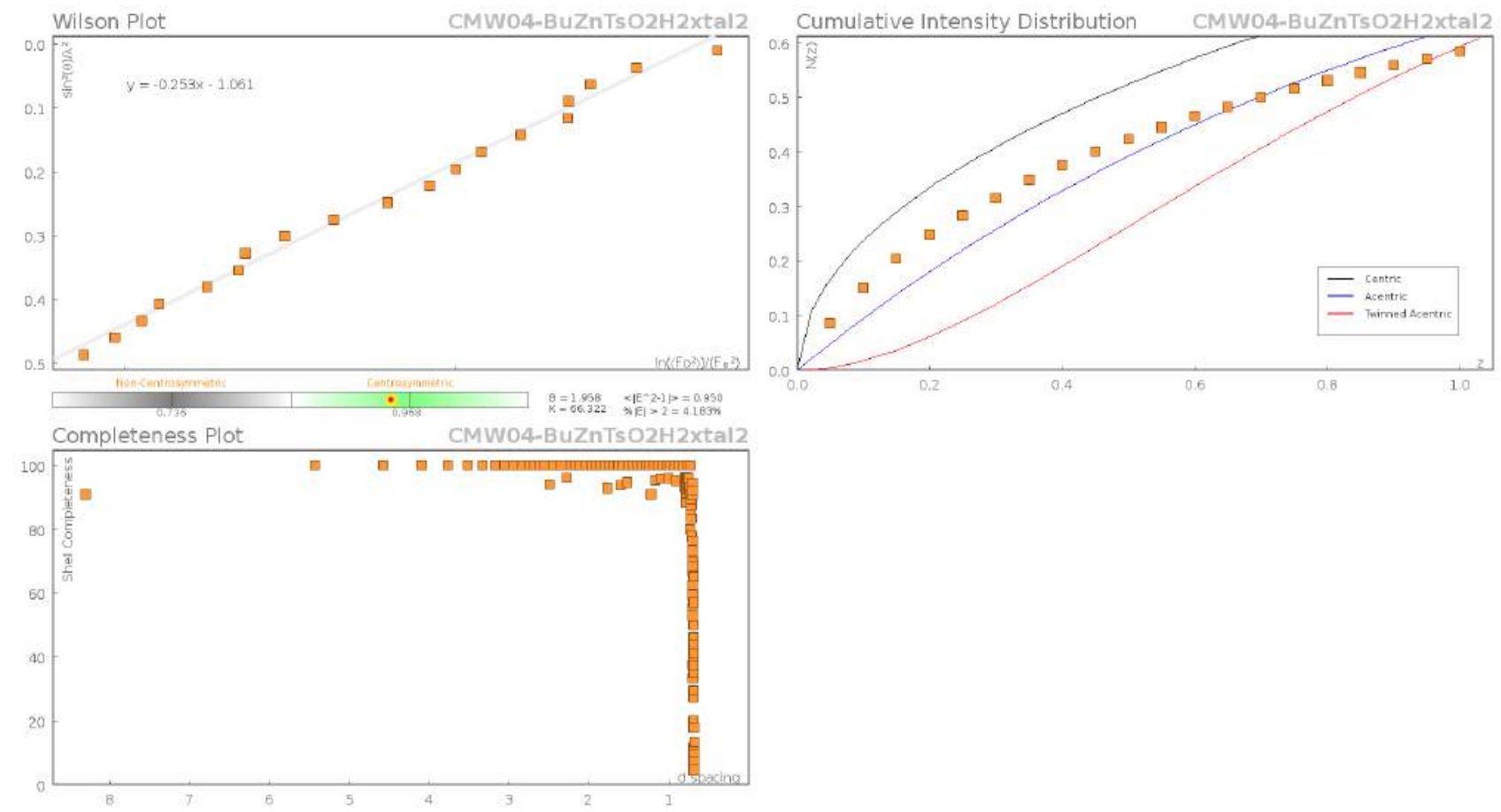
Data Plots: Refinement and Data
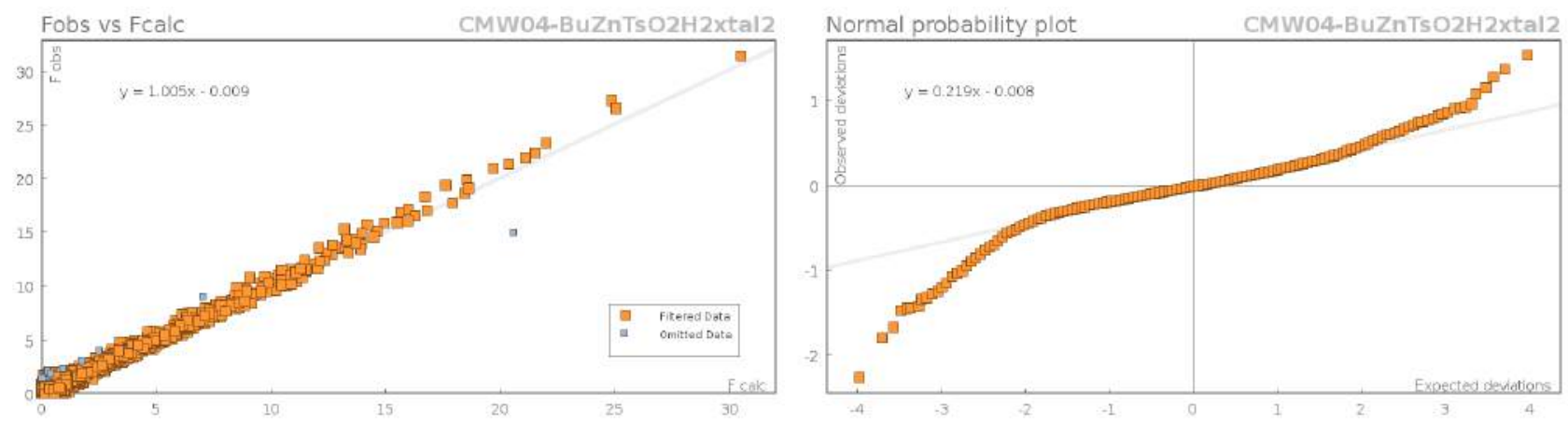

\section{Reflection Statistics}

$\begin{array}{ll}\begin{array}{l}\text { Total reflections (after } \\ \text { filtering) }\end{array} & 30830 \\ \text { Completeness } & 0.95 \\ \text { hklsub }>\text { max }</ \text { sub }>\text { collected } & (13,19,27) \\ \text { hkl }_{\max } \text { used } & (13,19,28) \\ \text { Lim } \mathrm{d}_{\max } \text { collected } & 100.0 \\ \mathrm{~d}_{\max } \text { used } & 12.68 \\ \text { Friedel pairs } & 9608 \\ \text { Inconsistent equivalents } & 0 \\ \mathrm{R}_{\text {sigma }} & 0.0668 \\ \text { Omitted reflections } & 0 \\ \text { Multiplicity } & (17230,6098,468) \\ \text { Removed systematic absences } & 0\end{array}$

$\begin{array}{ll}\text { Unique reflections } & 14175 \\ \text { Mean I/ } \sigma & 9.34 \\ \text { hklsub>min</sub> collected } & (-13,-19,-28) \\ \text { hkl }_{\text {min }} \text { used } & (-13,-19,0) \\ \text { Lim d } \mathrm{d}_{\text {min }} \text { collected } & 0.36 \\ \mathrm{~d}_{\text {min }} \text { used } & 0.69 \\ \text { Friedel pairs merged } & 1 \\ \text { Rint } & 0.0408 \\ \text { Intensity transformed } & 0 \\ \text { Omitted by user (OMIT hkl) } & 36 \\ \text { Maximum multiplicity } & 6 \\ \text { Filtered off (Shel/OMIT) } & 0\end{array}$

Images of the Crystal on the Diffractometer

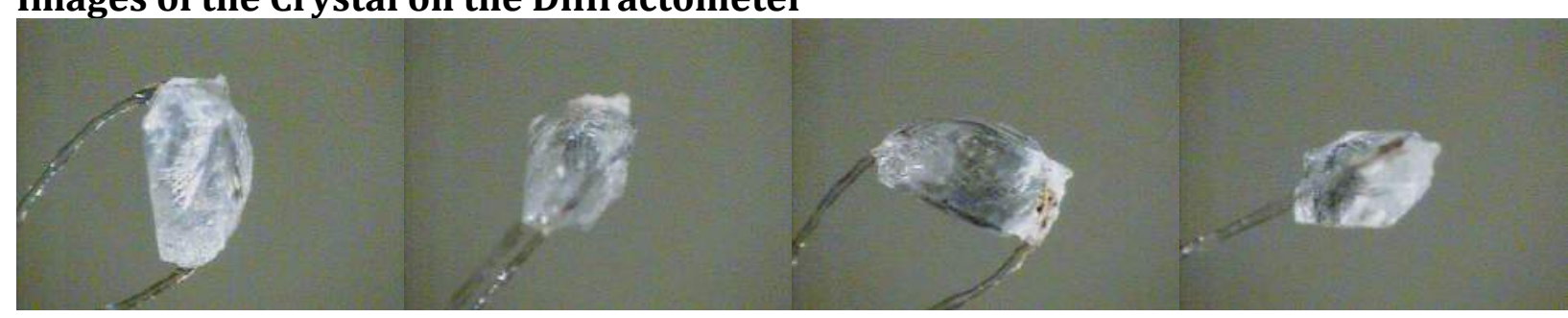

Table 1: Fractional Atomic Coordinates $\left(\times 10^{4}\right)$ and Equivalent Isotropic Displacement Parameters $\left(\AA^{2} \times 10^{3}\right)$ for CMW04-BuZnTs02H2xtal2. $U_{e q}$ is defined as $1 / 3$ of the trace of the orthogonalised $U_{i j}$.

\begin{tabular}{lccll}
\hline Atom & $\mathbf{x}$ & $\mathbf{y}$ & $\mathbf{z}$ & \multicolumn{1}{c}{$\boldsymbol{U}_{\boldsymbol{e q}}$} \\
\hline Zn1 & $6405.9(5)$ & $5378.5(4)$ & $7333.4(2)$ & $24.81(12)$ \\
S1 & $2987.3(10)$ & $5218.5(8)$ & $6548.5(5)$ & $24.13(19)$ \\
S2 & $6038.7(11)$ & $2908.5(7)$ & $7380.4(5)$ & $25.23(19)$ \\
N5A & $1212(6)$ & $2745(5)$ & $8843(3)$ & $38.3(9)$ \\
C28A & $486(10)$ & $2456(7)$ & $8092(3)$ & $39.8(12)$ \\
C29A & $940(30)$ & $1645(15)$ & $7704(4)$ & $38.6(12)$ \\
C30A & $440(20)$ & $1603(10)$ & $6920(4)$ & $43(2)$ \\
C31A & $580(40)$ & $640(20)$ & $6539(6)$ & $47(2)$ \\
C32A & $584(7)$ & $1828(7)$ & $9255(4)$ & $47.2(13)$ \\
C33A & $-1083(8)$ & $1458(9)$ & $9326(6)$ & $57.0(18)$ \\
C34A & $-1475(15)$ & $484(10)$ & $9732(7)$ & $70(2)$ \\
C35A & $-3017(14)$ & $154(12)$ & $9899(7)$ & $80(3)$ \\
C36A & $2940(8)$ & $3029(8)$ & $8928(3)$ & $46.6(13)$ \\
C37A & $3849(9)$ & $3552(9)$ & $9621(4)$ & $52.2(15)$ \\
C38A & $5565(10)$ & $3728(9)$ & $9649(6)$ & $61.5(19)$ \\
C39A & $5853(14)$ & $2724(11)$ & $9612(7)$ & $68(2)$
\end{tabular}




\begin{tabular}{|c|c|c|c|c|}
\hline Atom & $\mathbf{x}$ & $\mathbf{y}$ & $\mathbf{z}$ & $U_{e q}$ \\
\hline$\overline{\mathrm{C} 40 \mathrm{~A}}$ & $837(9)$ & $3679(6)$ & $9131(4)$ & $50.8(11)$ \\
\hline C41A & $-522(8)$ & $3999(6)$ & $8879(5)$ & $48.9(14)$ \\
\hline $\mathrm{C} 42 \mathrm{~A}$ & $-292(10)$ & $5133(6)$ & $9134(5)$ & $50.3(16)$ \\
\hline C43A & $921(12)$ & $5901(8)$ & $8797(6)$ & $50.7(17)$ \\
\hline N5B & $789(7)$ & $2549(4)$ & $8848(3)$ & $38.3(9)$ \\
\hline C28B & $865(9)$ & $2513(6)$ & $8085(3)$ & $39.8(12)$ \\
\hline C29B & $970(30)$ & $1511(12)$ & $7757(4)$ & $38.6(12)$ \\
\hline C30B & $207(17)$ & $1295(9)$ & 6992(4) & $43(2)$ \\
\hline C31B & $700(30)$ & $490(20)$ & $6607(6)$ & $47(2)$ \\
\hline C32B & $-642(9)$ & 1702(7) & $8978(3)$ & $47.2(13)$ \\
\hline C33B & $-957(12)$ & 1555(9) & $9702(4)$ & $57.0(18)$ \\
\hline C34B & $-2307(16)$ & $533(10)$ & $9715(5)$ & $70(2)$ \\
\hline C35B & $-2788(16)$ & $383(12)$ & 10386(6) & $80(3)$ \\
\hline C36B & $2175(7)$ & $2389(7)$ & $9259(4)$ & $46.6(13)$ \\
\hline C37B & $3724(7)$ & $3170(7)$ & $9217(5)$ & $52.2(15)$ \\
\hline C38B & $5021(9)$ & $2767(9)$ & $9505(6)$ & $61.5(19)$ \\
\hline C39B & $6529(11)$ & $3542(11)$ & $9480(7)$ & $68(2)$ \\
\hline C40B & $717(10)$ & $3617(5)$ & $9096(4)$ & $50.8(11)$ \\
\hline C41B & $1321(10)$ & $4630(5)$ & $8776(5)$ & $48.9(14)$ \\
\hline C42B & $468(11)$ & $5387(6)$ & $8908(6)$ & $50.3(16)$ \\
\hline C43B & 1323(12) & 6481(6) & $8722(6)$ & $50.7(17)$ \\
\hline S3A & 7865(5) & $7424(3)$ & $8452(3)$ & $47.6(5)$ \\
\hline O6A & $9322(9)$ & 8191(6) & $8765(4)$ & $55.2(17)$ \\
\hline O7A & $7073(10)$ & $6666(6)$ & $8895(4)$ & $50.8(15)$ \\
\hline N3A & $7979(10)$ & 6781(5) & $7775(4)$ & $32.1(10)$ \\
\hline $\mathrm{C} 21 \mathrm{~A}$ & $6635(10)$ & $8171(8)$ & $8224(7)$ & $43.5(11)$ \\
\hline $\mathrm{C} 22 \mathrm{~A}$ & $7190(13)$ & $9081(11)$ & $7906(10)$ & $48.3(13)$ \\
\hline $\mathrm{C} 23 \mathrm{~A}$ & $6277(14)$ & $9699(7)$ & $7754(6)$ & $43(2)$ \\
\hline C24A & $4834(11)$ & $9436(7)$ & $7923(6)$ & $39.2(13)$ \\
\hline $\mathrm{C} 25 \mathrm{~A}$ & $4286(12)$ & $8529(9)$ & $8249(9)$ & $47(2)$ \\
\hline $\mathrm{C} 26 \mathrm{~A}$ & $5218(16)$ & $7919(15)$ & $8411(16)$ & $49.2(11)$ \\
\hline $\mathrm{C} 27 \mathrm{~A}$ & $3855(13)$ & $10133(8)$ & $7789(7)$ & $48.4(19)$ \\
\hline S3B & $7995(5)$ & $7618(3)$ & $8406(3)$ & $47.6(5)$ \\
\hline O6B & $9445(8)$ & $8470(5)$ & $8554(4)$ & $55.2(17)$ \\
\hline O7B & $7564(9)$ & $6964(6)$ & $8964(4)$ & $50.8(15)$ \\
\hline N3B & 7861(10) & $6854(5)$ & $7741(4)$ & $32.1(10)$ \\
\hline $\mathrm{C} 21 \mathrm{~B}$ & $6569(9)$ & $8235(8)$ & $8251(6)$ & $43.5(11)$ \\
\hline $\mathrm{C} 22 \mathrm{~B}$ & $6874(12)$ & $9125(10)$ & $7900(9)$ & $48.3(13)$ \\
\hline C23B & $5794(13)$ & $9630(8)$ & $7791(6)$ & $48(3)$ \\
\hline C24B & $4429(10)$ & $9271(7)$ & $8034(6)$ & $39.2(13)$ \\
\hline C25B & $4084(11)$ & $8339(9)$ & $8346(7)$ & $46(3)$ \\
\hline C26B & $5164(15)$ & $7823(14)$ & $8444(15)$ & $49.2(11)$ \\
\hline C27B & $3275(12)$ & $9848(8)$ & $7949(6)$ & $48.4(19)$ \\
\hline $01^{\prime}$ & $5482(12)$ & $5138(10)$ & $8291(5)$ & $61.9(18)$ \\
\hline 02 & $1985(3)$ & $4262(2)$ & $6122.4(17)$ & $33.2(6)$ \\
\hline O2' & $4054(11)$ & $5199(9)$ & $8457(4)$ & $86(3)$ \\
\hline 03 & $2717(4)$ & $5323(3)$ & 7255.3(16) & $37.7(7)$ \\
\hline 04 & $4779(3)$ & $2904(2)$ & 7731.7(16) & $34.7(7)$ \\
\hline 05 & $6945(3)$ & $2268(2)$ & 7629.1(16) & $34.1(6)$ \\
\hline N1 & 7821(3) & $5723(2)$ & $6500.0(15)$ & $18.8(5)$ \\
\hline N2 & $7067(3)$ & $4086(2)$ & $7351.2(16)$ & $23.7(6)$ \\
\hline N4 & $4747(3)$ & $5412(3)$ & $6561.4(15)$ & $23.7(6)$ \\
\hline C1 & $8189(4)$ & $4760(3)$ & $6334.0(19)$ & $22.6(7)$ \\
\hline $\mathrm{C} 2$ & $8404(4)$ & $4234(3)$ & 6994(2) & $24.8(7)$ \\
\hline C3 & $8784(5)$ & $7405(3)$ & $7246(2)$ & $37.6(10)$ \\
\hline $\mathrm{C} 4$ & $9197(4)$ & $6629(3)$ & $6788(2)$ & $28.8(8)$ \\
\hline C5 & $6878(4)$ & $5977(3)$ & $5915.5(17)$ & $20.2(6)$ \\
\hline C6 & $5188(4)$ & $5320(3)$ & $5870.8(17)$ & $20.3(6)$ \\
\hline
\end{tabular}




\begin{tabular}{lclll}
\hline Atom & $\mathbf{x}$ & $\mathbf{y}$ & $\mathbf{z}$ & \multicolumn{1}{c}{$\boldsymbol{U}_{\boldsymbol{e q}}$} \\
\hline C7 & $2586(4)$ & $6270(3)$ & $6144.0(19)$ & $22.3(7)$ \\
C8 & $1673(4)$ & $6075(3)$ & $5493.1(19)$ & $24.7(7)$ \\
C9 & $1295(4)$ & $6881(3)$ & $5191(2)$ & $27.2(8)$ \\
C10 & $1834(4)$ & $7905(3)$ & $5526(2)$ & $28.3(8)$ \\
C11 & $2782(5)$ & $8098(3)$ & $6170(2)$ & $31.2(8)$ \\
C12 & $3158(4)$ & $7300(3)$ & $6482(2)$ & $28.8(8)$ \\
C13 & $1376(5)$ & $8768(4)$ & $5206(3)$ & $39.4(10)$ \\
C14 & $5208(4)$ & $2355(3)$ & $6512(2)$ & $23.0(7)$ \\
C15 & $3965(4)$ & $2606(3)$ & $6182(2)$ & $27.1(8)$ \\
C16 & $3367(4)$ & $2238(3)$ & $5496(2)$ & $28.7(8)$ \\
C17 & $3991(5)$ & $1622(3)$ & $5121(2)$ & $27.3(8)$ \\
C18 & $5214(4)$ & $1369(3)$ & $5459(2)$ & $28.2(8)$ \\
C19 & $5822(4)$ & $1726(3)$ & $6147(2)$ & $26.2(7)$ \\
C20 & $3358(5)$ & $1246(3)$ & $4365(2)$ & $36.0(9)$ \\
C1W & $4978(11)$ & $5137(11)$ & $8143(5)$ & $58.4(19)$
\end{tabular}

Table 2: Anisotropic Displacement Parameters $\left(\times 10^{4}\right)$ CMW04-BuZnTs02H2xtal2. The anisotropic displacement factor exponent takes the form: $-2 \pi^{2}\left[h^{2} a^{* 2} \times U_{11}+\ldots+2 h k a^{*} \times b^{*} \times U_{12}\right]$

\begin{tabular}{|c|c|c|c|c|c|c|}
\hline Atom & $U_{11}$ & $U_{22}$ & $U_{33}$ & $U_{23}$ & $U_{13}$ & $U_{12}$ \\
\hline$\overline{\mathrm{Zn} 1}$ & $25.7(2)$ & $30.4(2)$ & $16.8(2)$ & $0.45(16)$ & $1.29(15)$ & $8.65(17)$ \\
\hline S1 & $18.2(4)$ & $30.2(5)$ & $22.6(4)$ & $1.0(3)$ & $6.1(3)$ & $5.5(3)$ \\
\hline S2 & $24.6(4)$ & $25.3(4)$ & $24.0(4)$ & $4.1(3)$ & $5.3(3)$ & $5.3(3)$ \\
\hline N5A & $35.5(18)$ & $50.3(18)$ & $30.6(14)$ & $3.1(12)$ & $2.4(12)$ & $17.8(14)$ \\
\hline $\mathrm{C} 28 \mathrm{~A}$ & $33(3)$ & $58(2)$ & $34.9(15)$ & $6.7(13)$ & $7.5(14)$ & 22.1(18) \\
\hline $\mathrm{C} 29 \mathrm{~A}$ & $37(2)$ & $51(3)$ & $31.3(19)$ & $12.0(19)$ & $5.4(19)$ & $20(3)$ \\
\hline $\mathrm{C} 30 \mathrm{~A}$ & $40(5)$ & $60(6)$ & $31(2)$ & $15(2)$ & $9(2)$ & $18(5)$ \\
\hline C31A & $47(5)$ & $69(6)$ & $30(3)$ & $11(4)$ & $-2(3)$ & $28(3)$ \\
\hline $\mathrm{C} 32 \mathrm{~A}$ & $48(2)$ & $60(3)$ & $33(3)$ & $5(2)$ & $4.4(19)$ & $17(2)$ \\
\hline C33A & $53(3)$ & $75(4)$ & $32(3)$ & $0(3)$ & $5(3)$ & $9(3)$ \\
\hline C34A & $68(4)$ & $81(4)$ & $40(3)$ & $2(3)$ & $6(3)$ & $0(3)$ \\
\hline C35A & $77(5)$ & $90(6)$ & $45(4)$ & $-3(4)$ & $12(4)$ & $-7(4)$ \\
\hline C36A & $40(2)$ & $68(3)$ & $34(3)$ & $1(2)$ & $0.5(16)$ & $23.5(19)$ \\
\hline C37A & $43(2)$ & $77(4)$ & $34(3)$ & $-3(3)$ & $-3(2)$ & $22(2)$ \\
\hline C38A & $44(3)$ & $98(5)$ & $41(4)$ & $1(3)$ & $-6(2)$ & $28(3)$ \\
\hline C39A & $51(3)$ & $100(5)$ & $54(5)$ & $-1(4)$ & $-4(3)$ & $32(3)$ \\
\hline C40A & $51(3)$ & $60(2)$ & $51(3)$ & $5.9(17)$ & $19(2)$ & $25.5(19)$ \\
\hline C41A & $46(3)$ & $59(3)$ & $46(4)$ & $5(2)$ & $10(2)$ & $23(2)$ \\
\hline C42A & $50(4)$ & $59(3)$ & $46(4)$ & $2(3)$ & $8(3)$ & $24(3)$ \\
\hline $\mathrm{C} 43 \mathrm{~A}$ & $45(4)$ & $61(4)$ & $47(4)$ & $4(3)$ & $-2(3)$ & $22(3)$ \\
\hline N5B & $35.5(18)$ & $50.3(18)$ & $30.6(14)$ & $3.1(12)$ & $2.4(12)$ & $17.8(14)$ \\
\hline C28B & $33(3)$ & $58(2)$ & $34.9(15)$ & $6.7(13)$ & $7.5(14)$ & $22.1(18)$ \\
\hline C29B & $37(2)$ & $51(3)$ & $31.3(19)$ & $12.0(19)$ & $5.4(19)$ & $20(3)$ \\
\hline C30B & $40(5)$ & $60(6)$ & $31(2)$ & $15(2)$ & $9(2)$ & $18(5)$ \\
\hline C31B & $47(5)$ & $69(6)$ & $30(3)$ & $11(4)$ & $-2(3)$ & $28(3)$ \\
\hline C32B & $48(2)$ & $60(3)$ & $33(3)$ & $5(2)$ & 4.4(19) & $17(2)$ \\
\hline C33B & $53(3)$ & $75(4)$ & $32(3)$ & $0(3)$ & $5(3)$ & $9(3)$ \\
\hline C34B & $68(4)$ & $81(4)$ & $40(3)$ & $2(3)$ & $6(3)$ & $0(3)$ \\
\hline C35B & $77(5)$ & $90(6)$ & $45(4)$ & $-3(4)$ & $12(4)$ & $-7(4)$ \\
\hline C36B & $40(2)$ & $68(3)$ & $34(3)$ & $1(2)$ & $0.5(16)$ & $23.5(19)$ \\
\hline C37B & $43(2)$ & $77(4)$ & $34(3)$ & $-3(3)$ & $-3(2)$ & $22(2)$ \\
\hline C38B & $44(3)$ & $98(5)$ & $41(4)$ & $1(3)$ & $-6(2)$ & $28(3)$ \\
\hline C39B & $51(3)$ & $100(5)$ & $54(5)$ & $-1(4)$ & $-4(3)$ & $32(3)$ \\
\hline C40B & $51(3)$ & $60(2)$ & $51(3)$ & $5.9(17)$ & $19(2)$ & $25.5(19)$ \\
\hline C41B & $46(3)$ & $59(3)$ & $46(4)$ & $5(2)$ & $10(2)$ & $23(2)$ \\
\hline C42B & $50(4)$ & $59(3)$ & $46(4)$ & $2(3)$ & $8(3)$ & $24(3)$ \\
\hline C43B & $45(4)$ & $61(4)$ & $47(4)$ & $4(3)$ & $-2(3)$ & $22(3)$ \\
\hline S3A & $69.3(9)$ & $50.4(12)$ & $22.4(7)$ & $-11.9(8)$ & $-11.4(6)$ & $29.1(9)$ \\
\hline
\end{tabular}




\begin{tabular}{|c|c|c|c|c|c|c|}
\hline Atom & $U_{11}$ & $U_{22}$ & $\boldsymbol{U}_{33}$ & $U_{23}$ & $U_{13}$ & $U_{12}$ \\
\hline 06A & $70(2)$ & $60(3)$ & $25(3)$ & $-16(2)$ & $-18(2)$ & $21(2)$ \\
\hline O7A & $73(4)$ & $54(3)$ & $29(2)$ & $-5(2)$ & $-1(2)$ & 31(3) \\
\hline N3A & $38.1(17)$ & $33.3(15)$ & $23.5(14)$ & $-2.7(12)$ & $-2.7(12)$ & $14.1(13)$ \\
\hline $\mathrm{C} 21 \mathrm{~A}$ & $53.9(16)$ & $39.7(16)$ & $35.2(17)$ & $-6.1(13)$ & $-1.4(13)$ & $18.6(13)$ \\
\hline $\mathrm{C} 22 \mathrm{~A}$ & $59(4)$ & $38(2)$ & $44(3)$ & $-10.7(18)$ & $-3(3)$ & $18(2)$ \\
\hline $\mathrm{C} 23 \mathrm{~A}$ & $51(3)$ & $32(3)$ & $42(4)$ & $-5(3)$ & $3(3)$ & $12(2)$ \\
\hline $\mathrm{C} 24 \mathrm{~A}$ & $47(2)$ & $34(2)$ & $34(2)$ & $-2.3(16)$ & $0.8(18)$ & $12.8(18)$ \\
\hline C25A & $51(3)$ & $44(3)$ & $48(3)$ & $6.0(18)$ & $6.8(17)$ & $17.0(18)$ \\
\hline $\mathrm{C} 26 \mathrm{~A}$ & 71(3) & $43(3)$ & $38(3)$ & $0(2)$ & $4(2)$ & $28(2)$ \\
\hline C27A & $58(4)$ & $41(3)$ & $48(4)$ & $2(3)$ & $3(3)$ & $21(3)$ \\
\hline S3B & 69.3(9) & $50.4(12)$ & $22.4(7)$ & $-11.9(8)$ & $-11.4(6)$ & 29.1(9) \\
\hline O6B & $70(2)$ & $60(3)$ & $25(3)$ & $-16(2)$ & $-18(2)$ & $21(2)$ \\
\hline O7B & $73(4)$ & $54(3)$ & $29(2)$ & $-5(2)$ & $-1(2)$ & $31(3)$ \\
\hline N3B & $38.1(17)$ & $33.3(15)$ & $23.5(14)$ & $-2.7(12)$ & $-2.7(12)$ & $14.1(13)$ \\
\hline $\mathrm{C} 21 \mathrm{~B}$ & $53.9(16)$ & $39.7(16)$ & $35.2(17)$ & $-6.1(13)$ & $-1.4(13)$ & $18.6(13)$ \\
\hline C22B & $59(4)$ & $38(2)$ & $44(3)$ & $-10.7(18)$ & $-3(3)$ & $18(2)$ \\
\hline C23B & $49(3)$ & $47(3)$ & $50(3)$ & 1.8(19) & 6.1(19) & $19(2)$ \\
\hline C24B & $47(2)$ & $34(2)$ & $34(2)$ & $-2.3(16)$ & $0.8(18)$ & $12.8(18)$ \\
\hline C25B & $59(5)$ & $40(4)$ & $43(5)$ & $1(3)$ & $9(3)$ & $23(3)$ \\
\hline C26B & $71(3)$ & $43(3)$ & $38(3)$ & $0(2)$ & $4(2)$ & $28(2)$ \\
\hline C27B & $58(4)$ & $41(3)$ & $48(4)$ & $2(3)$ & $3(3)$ & $21(3)$ \\
\hline $01^{\prime}$ & $72(3)$ & $67(3)$ & $46(2)$ & $7(2)$ & $26(2)$ & $16(3)$ \\
\hline $\mathrm{O} 2$ & $22.8(13)$ & $26.1(14)$ & $45.8(18)$ & $-0.3(12)$ & $2.7(12)$ & $3.5(11)$ \\
\hline O2' & $88(4)$ & 118(8) & $68(6)$ & $26(6)$ & $42(4)$ & $42(5)$ \\
\hline 03 & $34.1(16)$ & $51.4(19)$ & $30.6(16)$ & $8.4(14)$ & 18.1(13) & $12.8(14)$ \\
\hline 04 & $35.1(16)$ & $33.8(15)$ & $33.0(16)$ & $5.1(12)$ & $15.9(13)$ & $4.1(13)$ \\
\hline 05 & $34.2(15)$ & $30.1(15)$ & $34.0(16)$ & $9.3(12)$ & $-0.9(12)$ & $7.2(12)$ \\
\hline N1 & $14.2(12)$ & $21.6(13)$ & $15.7(13)$ & $-1.6(10)$ & $0(1)$ & $1.1(10)$ \\
\hline $\mathrm{N} 2$ & $21.0(14)$ & $26.4(15)$ & $22.5(15)$ & $2.8(12)$ & $5.1(11)$ & $5.8(12)$ \\
\hline N4 & $19.2(14)$ & $35.0(17)$ & $15.4(14)$ & $1.1(12)$ & $4.5(11)$ & $6.8(12)$ \\
\hline C1 & $16.4(15)$ & $28.5(17)$ & $20.8(16)$ & $-0.1(13)$ & $3.9(12)$ & $4.9(13)$ \\
\hline $\mathrm{C} 2$ & $17.5(15)$ & $27.0(18)$ & 28.1(19) & $4.5(14)$ & $2.7(13)$ & $5.2(13)$ \\
\hline C3 & $37(2)$ & $28(2)$ & $34(2)$ & $-6.7(17)$ & $-7.1(18)$ & $0.3(17)$ \\
\hline $\mathrm{C} 4$ & 20.1(17) & $26.5(18)$ & $28.2(19)$ & $0.2(15)$ & $-4.8(14)$ & $-3.7(14)$ \\
\hline C5 & 18.4(15) & $25.1(16)$ & 14.1(15) & $0.9(12)$ & $1.3(11)$ & $3.9(13)$ \\
\hline C6 & 17.1(15) & 29.1(17) & $11.9(14)$ & $-0.5(12)$ & $1.4(11)$ & $4.6(13)$ \\
\hline C7 & $14.4(14)$ & $27.9(17)$ & $22.3(17)$ & $-1.8(13)$ & $2.6(12)$ & $5.0(13)$ \\
\hline C8 & $18.3(15)$ & $30.9(18)$ & $20.5(17)$ & $-5.7(14)$ & $2.7(13)$ & $3.8(14)$ \\
\hline C9 & $21.3(17)$ & $38(2)$ & $19.4(17)$ & $-1.9(15)$ & $1.4(13)$ & $7.2(15)$ \\
\hline C10 & $19.3(16)$ & $31.4(19)$ & $33(2)$ & $3.2(16)$ & $8.0(15)$ & $6.2(14)$ \\
\hline C11 & 24.8(18) & 26.5(19) & $38(2)$ & $-3.8(16)$ & $3.0(16)$ & $4.5(15)$ \\
\hline C12 & $16.3(16)$ & $41(2)$ & $23.4(18)$ & $-9.9(16)$ & $-1.9(13)$ & $6.5(15)$ \\
\hline C13 & $34(2)$ & $40(2)$ & $44(3)$ & $9(2)$ & $5.8(19)$ & $13.4(19)$ \\
\hline C14 & $18.9(15)$ & $21.1(16)$ & $26.8(18)$ & $2.5(13)$ & $5.1(13)$ & $3.4(13)$ \\
\hline C15 & $19.7(16)$ & $24.3(17)$ & $37(2)$ & $0.7(15)$ & $3.0(15)$ & $7.9(14)$ \\
\hline C16 & $23.2(17)$ & $23.0(17)$ & $36(2)$ & $2.4(15)$ & $-1.4(15)$ & $4.8(14)$ \\
\hline $\mathrm{C} 17$ & $28.4(18)$ & $17.2(16)$ & $30(2)$ & $1.3(14)$ & $2.8(15)$ & $0.7(14)$ \\
\hline C18 & $26.9(18)$ & $20.2(16)$ & $36(2)$ & $-0.5(15)$ & $6.1(15)$ & $5.5(14)$ \\
\hline C19 & $19.9(16)$ & $22.0(17)$ & $34(2)$ & $0.3(14)$ & $1.2(14)$ & $5.7(13)$ \\
\hline C20 & $40(2)$ & $28(2)$ & $33(2)$ & $-1.2(16)$ & $-2.2(18)$ & $6.2(17)$ \\
\hline 01W & $67(3)$ & $66(3)$ & $46(3)$ & $7(2)$ & $29(3)$ & $19(3)$ \\
\hline
\end{tabular}

Table 3: Bond Lengths in Å for CMW04-BuZnTs02H2xtal2.

\begin{tabular}{lll}
\hline Atom & Atom & Length/A \\
\hline Zn1 & N3A & $2.037(5)$ \\
Zn1 & N3B & $2.053(4)$ \\
Zn1 & O1' & $2.171(10)$
\end{tabular}

\begin{tabular}{lll}
\hline Atom & Atom & Length/Å \\
\hline $\mathrm{Zn} 1$ & $\mathrm{~N} 1$ & $2.219(3)$ \\
$\mathrm{Zn} 1$ & $\mathrm{~N} 2$ & $2.032(3)$ \\
$\mathrm{Zn} 1$ & $\mathrm{~N} 4$ & $2.028(3)$
\end{tabular}




\begin{tabular}{|c|c|c|c|c|c|}
\hline Atom & Atom & Length/Å & Atom & Atom & Length/Å \\
\hline$\overline{\mathrm{Zn} 1}$ & 01W & $2.185(10)$ & $\overline{\mathrm{N} 3 \mathrm{~A}}$ & $\mathrm{C} 3$ & $1.498(10)$ \\
\hline S1 & 02 & $1.457(3)$ & $\mathrm{C} 21 \mathrm{~A}$ & $\mathrm{C} 22 \mathrm{~A}$ & $1.383(8)$ \\
\hline S1 & 03 & $1.453(3)$ & $\mathrm{C} 21 \mathrm{~A}$ & C26A & $1.364(8)$ \\
\hline S1 & $\mathrm{N} 4$ & $1.576(3)$ & $\mathrm{C} 22 \mathrm{~A}$ & $\mathrm{C} 23 \mathrm{~A}$ & $1.391(10)$ \\
\hline S1 & $\mathrm{C} 7$ & $1.779(4)$ & C23A & C24A & $1.372(11)$ \\
\hline S2 & 04 & $1.444(3)$ & $\mathrm{C} 24 \mathrm{~A}$ & $\mathrm{C} 25 \mathrm{~A}$ & $1.389(10)$ \\
\hline S2 & 05 & $1.454(3)$ & $\mathrm{C} 24 \mathrm{~A}$ & $\mathrm{C} 27 \mathrm{~A}$ & $1.522(8)$ \\
\hline S2 & $\mathrm{N} 2$ & $1.573(3)$ & $\mathrm{C} 25 \mathrm{~A}$ & $\mathrm{C} 26 \mathrm{~A}$ & $1.400(8)$ \\
\hline S2 & C14 & $1.786(4)$ & S3B & 06B & $1.443(5)$ \\
\hline N5A & C28A & $1.509(6)$ & S3B & 07B & $1.459(5)$ \\
\hline N5A & C32A & $1.513(9)$ & S3B & N3B & $1.585(4)$ \\
\hline N5A & C36A & $1.517(8)$ & S3B & C21B & $1.791(5)$ \\
\hline N5A & $\mathrm{C} 40 \mathrm{~A}$ & $1.517(7)$ & N3B & $\mathrm{C} 3$ & $1.459(9)$ \\
\hline $\mathrm{C} 28 \mathrm{~A}$ & C29A & $1.504(4)$ & $\mathrm{C} 21 \mathrm{~B}$ & $\mathrm{C} 22 \mathrm{~B}$ & $1.383(8)$ \\
\hline $\mathrm{C} 29 \mathrm{~A}$ & $\mathrm{C} 30 \mathrm{~A}$ & $1.539(7)$ & $\mathrm{C} 21 \mathrm{~B}$ & $\mathrm{C} 26 \mathrm{~B}$ & $1.365(8)$ \\
\hline C30A & C31A & $1.521(9)$ & $\mathrm{C} 22 \mathrm{~B}$ & C23B & $1.391(10)$ \\
\hline $\mathrm{C} 32 \mathrm{~A}$ & C33A & $1.503(4)$ & $\mathrm{C} 23 \mathrm{~B}$ & $\mathrm{C} 24 \mathrm{~B}$ & $1.372(11)$ \\
\hline C33A & C34A & $1.539(12)$ & $\mathrm{C} 24 \mathrm{~B}$ & $\mathrm{C} 25 \mathrm{~B}$ & $1.389(10)$ \\
\hline C34A & C35A & $1.454(13)$ & C24B & C27B & $1.522(8)$ \\
\hline $\mathrm{C} 36 \mathrm{~A}$ & C37A & $1.504(4)$ & C25B & $\mathrm{C} 26 \mathrm{~B}$ & $1.401(8)$ \\
\hline C37A & C38A & $1.537(11)$ & $01^{\prime}$ & $02^{\prime}$ & $1.445(14)$ \\
\hline C38A & C39A & $1.465(11)$ & N1 & $\mathrm{C} 1$ & $1.480(5)$ \\
\hline C40A & $\mathrm{C} 41 \mathrm{~A}$ & $1.502(4)$ & N1 & C4 & $1.472(4)$ \\
\hline C41A & C42A & $1.521(4)$ & N1 & C5 & $1.474(4)$ \\
\hline C42A & C43A & $1.520(4)$ & N2 & $\mathrm{C} 2$ & $1.481(5)$ \\
\hline N5B & C28B & $1.510(6)$ & $\mathrm{N} 4$ & C6 & $1.482(4)$ \\
\hline N5B & C32B & $1.511(9)$ & $\mathrm{C} 1$ & $\mathrm{C} 2$ & $1.529(5)$ \\
\hline N5B & C36B & $1.514(8)$ & $\mathrm{C} 3$ & $\mathrm{C} 4$ & $1.522(6)$ \\
\hline N5B & C40B & $1.521(7)$ & $\mathrm{C} 5$ & C6 & $1.526(5)$ \\
\hline C28B & C29B & $1.503(4)$ & $\mathrm{C} 7$ & $\mathrm{C} 8$ & $1.396(5)$ \\
\hline C29B & C30B & $1.539(7)$ & $\mathrm{C} 7$ & $\mathrm{C} 12$ & $1.405(5)$ \\
\hline C30B & C31B & $1.521(9)$ & $\mathrm{C} 8$ & C9 & $1.387(6)$ \\
\hline C32B & C33B & $1.502(4)$ & C9 & C10 & $1.398(6)$ \\
\hline C33B & C34B & $1.539(12)$ & $\mathrm{C} 10$ & C11 & $1.400(6)$ \\
\hline C34B & C35B & $1.454(13)$ & C10 & C13 & $1.504(6)$ \\
\hline C36B & C37B & $1.503(4)$ & C11 & C12 & $1.383(6)$ \\
\hline C37B & C38B & $1.537(11)$ & C14 & C15 & $1.400(5)$ \\
\hline C38B & C39B & $1.465(11)$ & C14 & C19 & $1.391(5)$ \\
\hline $\mathrm{C} 40 \mathrm{~B}$ & $\mathrm{C} 41 \mathrm{~B}$ & $1.505(4)$ & C15 & C16 & $1.385(6)$ \\
\hline C41B & $\mathrm{C} 42 \mathrm{~B}$ & $1.520(4)$ & C16 & $\mathrm{C} 17$ & $1.396(6)$ \\
\hline C42B & C43B & $1.520(4)$ & $\mathrm{C} 17$ & C18 & $1.391(6)$ \\
\hline S3A & $06 \mathrm{~A}$ & $1.444(5)$ & $\mathrm{C} 17$ & $\mathrm{C} 20$ & $1.513(6)$ \\
\hline S3A & 07A & $1.457(5)$ & C18 & C19 & $1.386(6)$ \\
\hline S3A & N3A & $1.585(4)$ & & & \\
\hline S3A & $\mathrm{C} 21 \mathrm{~A}$ & $1.792(5)$ & & & \\
\hline
\end{tabular}

Table 4: Bond Angles in ${ }^{\circ}$ for CMW04-BuZnTsO2H2xtal2.

\begin{tabular}{llll}
\hline Atom & Atom & Atom & \multicolumn{1}{c}{ Angle $^{\circ}$} \\
\hline N3A & Zn1 & N1 & $81.4(2)$ \\
N3A & Zn1 & O1W & $96.8(4)$ \\
N3B & Zn1 & O1' & $90.6(4)$ \\
N3B & Zn1 & N1 & $81.7(2)$ \\
O1' & Zn1 & N1 & $168.0(3)$ \\
N2 & Zn1 & N3A & $117.4(4)$ \\
N2 & Zn1 & N3B & $122.3(3)$ \\
N2 & Zn1 & O1' & $94.1(4)$
\end{tabular}

\begin{tabular}{llll}
\hline Atom & Atom & Atom & \multicolumn{1}{c}{ Angle $^{\circ}$} \\
\hline N2 & Zn1 & N1 & $82.34(12)$ \\
N2 & Zn1 & O1W & $101.5(4)$ \\
N4 & Zn1 & N3A & $117.9(4)$ \\
N4 & Zn1 & N3B & $113.1(3)$ \\
N4 & Zn1 & O1' & $109.7(3)$ \\
N4 & Zn1 & N1 & $81.98(11)$ \\
N4 & Zn1 & N2 & $118.85(13)$ \\
N4 & Zn1 & O1W & $96.0(2)$
\end{tabular}




\begin{tabular}{|c|c|c|c|}
\hline Atom & Atom & Atom & Angle ${ }^{\circ}$ \\
\hline $\mathrm{O} 1 \mathrm{~W}$ & $\mathrm{Zn} 1$ & N1 & $176.1(4)$ \\
\hline 02 & S1 & N4 & $113.57(17)$ \\
\hline 02 & S1 & $\mathrm{C} 7$ & $105.10(18)$ \\
\hline 03 & S1 & 02 & $115.77(19)$ \\
\hline 03 & S1 & N4 & $108.65(18)$ \\
\hline 03 & S1 & C7 & $106.28(18)$ \\
\hline N4 & S1 & C7 & $106.78(17)$ \\
\hline 04 & $\mathrm{~S} 2$ & 05 & $117.22(19)$ \\
\hline 04 & S2 & N2 & $108.60(18)$ \\
\hline 04 & S2 & C14 & $106.29(18)$ \\
\hline 05 & S2 & $\mathrm{N} 2$ & $111.96(18)$ \\
\hline 05 & S2 & C14 & $104.80(18)$ \\
\hline N2 & S2 & C14 & $107.33(17)$ \\
\hline C28A & N5A & C32A & $110.1(4)$ \\
\hline $\mathrm{C} 28 \mathrm{~A}$ & N5A & C36A & 111.1(4) \\
\hline $\mathrm{C} 28 \mathrm{~A}$ & N5A & C40A & $109.5(4)$ \\
\hline $\mathrm{C} 32 \mathrm{~A}$ & N5A & C36A & $108.6(4)$ \\
\hline C32A & N5A & $\mathrm{C} 40 \mathrm{~A}$ & $108.4(5)$ \\
\hline C40A & N5A & C36A & $109.1(5)$ \\
\hline C29A & C28A & N5A & $115.8(5)$ \\
\hline C28A & C29A & C30A & $111.0(4)$ \\
\hline C31A & C30A & C29A & 111.1(5) \\
\hline C33A & C32A & N5A & $119.9(5)$ \\
\hline $\mathrm{C} 32 \mathrm{~A}$ & C33A & C34A & $110.7(7)$ \\
\hline C35A & C34A & C33A & $114.2(9)$ \\
\hline C37A & C36A & N5A & $117.0(5)$ \\
\hline C36A & C37A & C38A & $111.4(6)$ \\
\hline C39A & C38A & C37A & 111.3(7) \\
\hline $\mathrm{C} 41 \mathrm{~A}$ & $\mathrm{C} 40 \mathrm{~A}$ & N5A & $127.2(5)$ \\
\hline $\mathrm{C} 40 \mathrm{~A}$ & $\mathrm{C} 41 \mathrm{~A}$ & $\mathrm{C} 42 \mathrm{~A}$ & $112.5(5)$ \\
\hline C43A & $\mathrm{C} 42 \mathrm{~A}$ & $\mathrm{C} 41 \mathrm{~A}$ & $110.8(5)$ \\
\hline C28B & N5B & C32B & $110.1(4)$ \\
\hline C28B & N5B & C36B & $111.3(4)$ \\
\hline C28B & N5B & C40B & $108.9(4)$ \\
\hline C32B & N5B & C36B & $108.9(4)$ \\
\hline C32B & N5B & C40B & 108.3(5) \\
\hline C36B & N5B & C40B & $109.2(5)$ \\
\hline C29B & C28B & N5B & $115.9(5)$ \\
\hline C28B & C29B & C30B & $111.0(4)$ \\
\hline C31B & C30B & C29B & 111.1(5) \\
\hline C33B & C32B & N5B & $120.3(6)$ \\
\hline C32B & C33B & C34B & $110.7(7)$ \\
\hline C35B & C34B & C33B & $114.2(9)$ \\
\hline C37B & C36B & N5B & $117.4(5)$ \\
\hline C36B & C37B & C38B & $111.5(6)$ \\
\hline C39B & C38B & C37B & 111.3(7) \\
\hline C41B & C40B & N5B & $126.4(5)$ \\
\hline C40B & C41B & C42B & $112.5(5)$ \\
\hline C41B & C42B & C43B & $110.8(5)$ \\
\hline O6A & S3A & 07A & $116.6(4)$ \\
\hline 06A & S3A & N3A & $112.5(3)$ \\
\hline 06A & S3A & $\mathrm{C} 21 \mathrm{~A}$ & $105.4(3)$ \\
\hline 07A & S3A & N3A & $107.8(3)$ \\
\hline 07A & S3A & $\mathrm{C} 21 \mathrm{~A}$ & $105.2(3)$ \\
\hline N3A & S3A & C21A & $108.9(2)$ \\
\hline S3A & N3A & Zn1 & 125.1(4) \\
\hline C3 & N3A & Zn1 & $111.4(4)$ \\
\hline C3 & N3A & S3A & 117.1(4) \\
\hline
\end{tabular}

\begin{tabular}{|c|c|c|c|}
\hline Atom & Atom & Atom & Angle $/^{\circ}$ \\
\hline$\overline{\mathrm{C} 22 \mathrm{~A}}$ & C21A & S3A & $119.1(5)$ \\
\hline C26A & C21A & S3A & $121.5(4)$ \\
\hline C26A & C21A & C22A & $119.1(5)$ \\
\hline C21A & $\mathrm{C} 22 \mathrm{~A}$ & C23A & $120.0(7)$ \\
\hline C24A & C23A & $\mathrm{C} 22 \mathrm{~A}$ & $121.3(6)$ \\
\hline C23A & C24A & C25A & $118.6(6)$ \\
\hline C23A & C24A & C27A & $121.8(7)$ \\
\hline $\mathrm{C} 25 \mathrm{~A}$ & $\mathrm{C} 24 \mathrm{~A}$ & C27A & $119.6(7)$ \\
\hline $\mathrm{C} 24 \mathrm{~A}$ & C25A & $\mathrm{C} 26 \mathrm{~A}$ & $119.8(7)$ \\
\hline $\mathrm{C} 21 \mathrm{~A}$ & C26A & C25A & $121.0(6)$ \\
\hline O6B & S3B & 07B & $116.5(3)$ \\
\hline O6B & S3B & N3B & $112.8(3)$ \\
\hline O6B & S3B & C21B & $105.6(3)$ \\
\hline O7B & S3B & N3B & $107.6(3)$ \\
\hline O7B & S3B & $\mathrm{C} 21 \mathrm{~B}$ & $105.1(3)$ \\
\hline N3B & S3B & C21B & $108.9(2)$ \\
\hline S3B & N3B & Zn1 & $134.7(4)$ \\
\hline C3 & N3B & $\mathrm{Zn} 1$ & $112.4(4)$ \\
\hline C3 & N3B & S3B & $111.7(4)$ \\
\hline C22B & C21B & S3B & $119.2(5)$ \\
\hline C26B & C21B & S3B & $121.7(4)$ \\
\hline C26B & $\mathrm{C} 21 \mathrm{~B}$ & $\mathrm{C} 22 \mathrm{~B}$ & 119.1(5) \\
\hline C21B & $\mathrm{C} 22 \mathrm{~B}$ & C23B & $119.9(7)$ \\
\hline C24B & C23B & C22B & $121.3(6)$ \\
\hline C23B & C24B & C25B & $118.6(6)$ \\
\hline C23B & C24B & C27B & $121.7(7)$ \\
\hline $\mathrm{C} 25 \mathrm{~B}$ & $\mathrm{C} 24 \mathrm{~B}$ & C27B & $119.7(7)$ \\
\hline $\mathrm{C} 24 \mathrm{~B}$ & C25B & C26B & $119.8(7)$ \\
\hline C21B & C26B & C25B & $121.0(6)$ \\
\hline O2' & $01^{\prime}$ & Zn1 & $129.7(8)$ \\
\hline $\mathrm{C} 1$ & $\mathrm{~N} 1$ & Zn1 & $106.5(2)$ \\
\hline $\mathrm{C} 4$ & N1 & Zn1 & $106.0(2)$ \\
\hline $\mathrm{C} 4$ & N1 & C1 & $112.4(3)$ \\
\hline $\mathrm{C} 4$ & N1 & C5 & $112.3(3)$ \\
\hline C5 & N1 & Zn1 & $106.3(2)$ \\
\hline $\mathrm{C} 5$ & N1 & C1 & $112.8(3)$ \\
\hline $\mathrm{S} 2$ & N2 & $\mathrm{Zn} 1$ & $127.72(18)$ \\
\hline $\mathrm{C} 2$ & N2 & $\mathrm{Zn} 1$ & $110.8(2)$ \\
\hline $\mathrm{C} 2$ & N2 & S2 & 115.6(3) \\
\hline S1 & $\mathrm{N} 4$ & $\mathrm{Zn} 1$ & $131.15(18)$ \\
\hline C6 & N4 & Zn1 & $112.0(2)$ \\
\hline C6 & N4 & S1 & $114.6(2)$ \\
\hline N1 & $\mathrm{C} 1$ & $\mathrm{C} 2$ & $109.5(3)$ \\
\hline N2 & C2 & C1 & $109.7(3)$ \\
\hline N3A & C3 & C4 & $106.0(4)$ \\
\hline N3B & C3 & C4 & $110.4(4)$ \\
\hline N1 & C4 & C3 & $110.5(3)$ \\
\hline N1 & $\mathrm{C} 5$ & C6 & $110.9(3)$ \\
\hline N4 & C6 & C5 & $108.7(3)$ \\
\hline C8 & $\mathrm{C} 7$ & S1 & $120.2(3)$ \\
\hline C8 & $\mathrm{C} 7$ & $\mathrm{C} 12$ & $119.4(4)$ \\
\hline C12 & C7 & S1 & $120.5(3)$ \\
\hline C9 & $\mathrm{C} 8$ & $\mathrm{C} 7$ & $120.4(3)$ \\
\hline C8 & $\mathrm{C} 9$ & C10 & $120.8(4)$ \\
\hline C9 & C10 & C11 & $118.3(4)$ \\
\hline C9 & C10 & $\mathrm{C} 13$ & $120.5(4)$ \\
\hline C11 & C10 & C13 & $121.2(4)$ \\
\hline C12 & C11 & C10 & $121.6(4)$ \\
\hline
\end{tabular}




\begin{tabular}{|c|c|c|c|c|c|c|c|}
\hline Atom & Atom & Atom & Angle $/^{\circ}$ & Atom & Atom & Atom & Angle $/^{\circ}$ \\
\hline$\overline{\mathrm{C} 11}$ & C12 & C7 & $119.6(4)$ & C16 & C17 & C20 & $120.7(4)$ \\
\hline C15 & C14 & S2 & 119.1(3) & C18 & C17 & C16 & $118.3(4)$ \\
\hline C19 & C14 & S2 & $121.3(3)$ & C18 & C17 & C20 & $121.0(4)$ \\
\hline C19 & C14 & C15 & $119.5(4)$ & C19 & C18 & C17 & $121.5(4)$ \\
\hline C16 & C15 & C14 & $119.9(4)$ & C18 & C19 & C14 & $119.8(3)$ \\
\hline C15 & C16 & C17 & $121.0(4)$ & & & & \\
\hline
\end{tabular}

Table 5: Hydrogen Fractional Atomic Coordinates $\left(\times 10^{4}\right)$ and Equivalent Isotropic Displacement Parameters $\left(\AA^{2} \times 10^{3}\right)$ for CMW04-BuZnTsO2H2xtal2. $U_{e q}$ is defined as $1 / 3$ of the trace of the orthogonalised $U_{i j}$.

\begin{tabular}{|c|c|c|c|c|c|}
\hline Atom & & & & & $U_{e q}$ \\
\hline$\overline{\mathrm{H} 28 \mathrm{~A}}$ & -642 & 2190 & 8065 & 48 & \\
\hline H28B & 751 & 3106 & 7853 & 48 & \\
\hline H29A & 454 & 943 & 7859 & 46 & \\
\hline H29B & 2065 & 1821 & 7809 & 46 & \\
\hline $\mathrm{H} 30 \mathrm{~A}$ & -636 & 1584 & 6825 & 51 & \\
\hline H30B & 1091 & 2247 & 6748 & 51 & \\
\hline $\mathrm{H} 31 \mathrm{~A}$ & 197 & 605 & 6045 & 71 & \\
\hline H31B & -33 & -2 & 6723 & 71 & \\
\hline H31C & 1654 & 679 & 6605 & 71 & \\
\hline H32A & 799 & 1218 & 9048 & 57 & \\
\hline H32B & 1187 & 2015 & 9728 & 57 & \\
\hline H33A & -1721 & 1278 & 8861 & 68 & \\
\hline H33B & -1318 & 2032 & 9570 & 68 & \\
\hline H34A & -1353 & -110 & 9456 & 84 & \\
\hline H34B & -730 & 640 & 10167 & 84 & \\
\hline H35A & -3758 & -196 & 9483 & 120 & \\
\hline H35B & -3237 & 774 & 10073 & 120 & \\
\hline H35C & -3095 & -336 & 10255 & 120 & \\
\hline H36A & 3168 & 2375 & 8827 & 56 & \\
\hline H36B & 3312 & 3503 & 8574 & 56 & \\
\hline H37A & 3462 & 3105 & 9986 & 63 & \\
\hline H37B & 3708 & 4239 & 9715 & 63 & \\
\hline H38A & 5930 & 4119 & 9258 & 74 & \\
\hline H38B & 6148 & 4162 & 10084 & 74 & \\
\hline H39A & 5629 & 2419 & 9129 & 103 & \\
\hline H39B & 5193 & 2236 & 9884 & 103 & \\
\hline H39C & 6930 & 2846 & 9799 & 103 & \\
\hline $\mathrm{H} 40 \mathrm{~A}$ & 807 & 3592 & 9627 & 61 & \\
\hline H40B & 1748 & 4305 & 9115 & 61 & \\
\hline H41A & -1439 & 3523 & 9038 & 59 & \\
\hline H41B & -714 & 3916 & 8367 & 59 & \\
\hline $\mathrm{H} 42 \mathrm{~A}$ & -1273 & 5267 & 9026 & 60 & \\
\hline H42B & 28 & 5244 & 9643 & 60 & \\
\hline H43A & 591 & 5805 & 8295 & 76 & \\
\hline H43B & 1892 & 5769 & 8904 & 76 & \\
\hline H43C & 1062 & 6626 & 8974 & 76 & \\
\hline H28C & -57 & 2628 & 7840 & 48 & \\
\hline H28D & 1770 & 3108 & 8012 & 48 & \\
\hline H29C & 452 & 914 & 8009 & 46 & \\
\hline H29D & 2057 & 1569 & 7791 & 46 & \\
\hline H30C & -922 & 1032 & 6961 & 51 & \\
\hline H30D & 497 & 1961 & 6772 & 51 & \\
\hline H31D & 271 & 408 & 6114 & 71 & \\
\hline H31E & 319 & -194 & 6794 & 71 & \\
\hline H31F & 1819 & 723 & 6664 & 71 & \\
\hline H32C & -1530 & 1835 & 8714 & 57 & \\
\hline
\end{tabular}




\begin{tabular}{|c|c|c|c|c|}
\hline Atom & & J & & $U_{e q}$ \\
\hline H32D & -628 & 1021 & 8772 & 57 \\
\hline H33C & -1201 & 2165 & 9890 & 68 \\
\hline H33D & -30 & 1525 & 9999 & 68 \\
\hline H34C & -3190 & 536 & 9374 & 84 \\
\hline H34D & -2011 & -75 & 9566 & 84 \\
\hline H35D & -3613 & -298 & 10358 & 120 \\
\hline H35E & -3158 & 952 & 10522 & 120 \\
\hline H35F & -1916 & 393 & 10730 & 120 \\
\hline H36C & 2047 & 2391 & 9751 & 56 \\
\hline H36D & 2174 & 1678 & 9105 & 56 \\
\hline H37C & 3854 & 3847 & 9482 & 63 \\
\hline H37D & 3787 & 3301 & 8728 & 63 \\
\hline H38C & 4943 & 2619 & 9990 & 74 \\
\hline H38D & 4907 & 2100 & 9233 & 74 \\
\hline H39D & 6632 & 3658 & 8997 & 103 \\
\hline H39E & 7332 & 3278 & 9685 & 103 \\
\hline H39F & 6633 & 4210 & 9739 & 103 \\
\hline $\mathrm{H} 40 \mathrm{C}$ & -381 & 3503 & 9102 & 61 \\
\hline H40D & 1225 & 3767 & 9585 & 61 \\
\hline $\mathrm{H} 41 \mathrm{C}$ & 1229 & 4468 & 8271 & 59 \\
\hline H41D & 2422 & 4977 & 8966 & 59 \\
\hline $\mathrm{H} 42 \mathrm{C}$ & 358 & 5430 & 9402 & 60 \\
\hline H42D & -572 & 5118 & 8628 & 60 \\
\hline H43D & 1528 & 6428 & 8248 & 76 \\
\hline H43E & 2296 & 6786 & 9041 & 76 \\
\hline $\mathrm{H} 43 \mathrm{~F}$ & 697 & 6932 & 8757 & 76 \\
\hline $\mathrm{H} 22 \mathrm{~A}$ & 8193 & 9284 & 7793 & 58 \\
\hline $\mathrm{H} 23 \mathrm{~A}$ & 6660 & 10315 & 7528 & 52 \\
\hline $\mathrm{H} 25 \mathrm{~A}$ & 3281 & 8323 & 8362 & 57 \\
\hline $\mathrm{H} 26 \mathrm{~A}$ & 4858 & 7320 & 8655 & 59 \\
\hline $\mathrm{H} 27 \mathrm{~A}$ & 4201 & 10731 & 8149 & 73 \\
\hline H27B & 2779 & 9721 & 7798 & 73 \\
\hline $\mathrm{H} 27 \mathrm{C}$ & 3953 & 10394 & 7336 & 73 \\
\hline H22B & 7819 & 9391 & 7733 & 58 \\
\hline H23B & 6007 & 10234 & 7543 & 58 \\
\hline H25B & 3119 & 8052 & 8493 & 55 \\
\hline H26B & 4914 & 7177 & 8648 & 59 \\
\hline H27D & 3815 & 10600 & 7925 & 73 \\
\hline H27E & 2727 & 9764 & 8344 & 73 \\
\hline $\mathrm{H} 27 \mathrm{~F}$ & 2540 & 9554 & 7522 & 73 \\
\hline H1A & 9140 & 4948 & 6129 & 27 \\
\hline H1B & 7346 & 4265 & 5992 & 27 \\
\hline $\mathrm{H} 2 \mathrm{~A}$ & 8509 & 3541 & 6876 & 30 \\
\hline $\mathrm{H} 2 \mathrm{~B}$ & 9351 & 4680 & 7303 & 30 \\
\hline H3A & 9720 & 7982 & 7473 & 45 \\
\hline H3B & 8103 & 7716 & 6970 & 45 \\
\hline $\mathrm{H} 4 \mathrm{~A}$ & 9669 & 6989 & 6407 & 35 \\
\hline $\mathrm{H} 4 \mathrm{~B}$ & 9956 & 6378 & 7063 & 35 \\
\hline H5A & 7230 & 5836 & 5479 & 24 \\
\hline H5B & 7005 & 6736 & 5975 & 24 \\
\hline H6A & 4552 & 5579 & 5527 & 24 \\
\hline H6B & 5020 & 4573 & 5722 & 24 \\
\hline H8 & 1309 & 5385 & 5256 & 30 \\
\hline H9 & 662 & 6736 & 4750 & 33 \\
\hline H11 & 3177 & 8794 & 6400 & 37 \\
\hline H12 & 3798 & 7447 & 6920 & 35 \\
\hline $\mathrm{H} 13 \mathrm{~A}$ & 1288 & 8663 & 4703 & 59 \\
\hline H13B & 2155 & 9453 & 5382 & 59 \\
\hline
\end{tabular}




\begin{tabular}{|c|c|c|c|c|}
\hline Atom & $\mathbf{x}$ & $\mathbf{y}$ & $\mathbf{z}$ & $U_{e q}$ \\
\hline H13C & 386 & 8751 & 5326 & 59 \\
\hline H15 & 3531 & 3029 & 6429 & 32 \\
\hline H16 & 2518 & 2407 & 5276 & 34 \\
\hline H18 & 5642 & 942 & 5213 & 34 \\
\hline H19 & 6655 & 1542 & 6368 & 31 \\
\hline $\mathrm{H} 20 \mathrm{~A}$ & 2267 & 1165 & 4275 & 54 \\
\hline $\mathrm{H} 20 \mathrm{~B}$ & 3483 & 566 & 4253 & 54 \\
\hline H20C & 3915 & 1765 & 4079 & 54 \\
\hline H1WA & 4161 & 5424 & 7947 & 88 \\
\hline H1WB & 5592 & 5698 & 8512 & 88 \\
\hline H2' & 3304 & 5101 & 8022 & 70 \\
\hline H1' & $6040(140)$ & $5730(50)$ & $8660(40)$ & 88 \\
\hline
\end{tabular}

Table 6: Hydrogen Bond information for CMW04-BuZnTsO2H2xtal2.

\begin{tabular}{lllcccc}
\hline $\mathbf{D}$ & $\mathbf{H}$ & $\mathbf{A}$ & $\mathbf{d}(\mathbf{D}-\mathbf{H}) / \AA$ & $\mathbf{d}(\mathbf{H}-\mathbf{A}) / \AA$ & $\mathbf{d}(\mathbf{D}-\mathbf{A}) / \AA$ & $\mathbf{D}-\mathbf{H}-\mathbf{A} / \mathbf{d e g}$ \\
\hline C41B & H41D & O2' & 0.99 & 1.89 & $2.595(12)$ & 125.5 \\
O1W & H1WA & O3 & 1.01 & 1.74 & $2.636(9)$ & 145.7 \\
O1W & H1WB & O7A & 0.99 & 1.62 & $2.552(15)$ & 156.6 \\
O2' & H2' & O3 & 1.00 & 1.61 & $2.552(9)$ & 155.9 \\
O1' $^{\prime}$ & H1' & O7B & $0.995(2)$ & $1.81(6)$ & $2.723(15)$ & $151(10)$
\end{tabular}

\section{References}

1. $\quad$ Motekaitis, R. J.; Martell, A. E.; Murase, I. Inorg. Chem. 1986, 25, 938-944.

2. (a) Bruker-AXS APEX2. Version 2014.11-0, Madison, WI, 2014; (b) Sheldrick, G. Acta Cryst. 2008, A64, 112-122; (c) Dolomanov, O. V.; Bourhis, L. J.; Gildea, R. J.; Howard, J. A. K.; Puschmann, H. J. Appl. Cryst. 2009, 42, 339-341; (d) Bruker-AXS SAINT-8.34A-2013 - Software for the Integration of CCD Detector System Bruker Analytical X-ray Systems, Madison, WI, 2013; (e) Sheldrick, G. Acta Cryst. 2015, A71, 3-8. 